\title{
Electrographic signatures of postanoxic brain injury
}
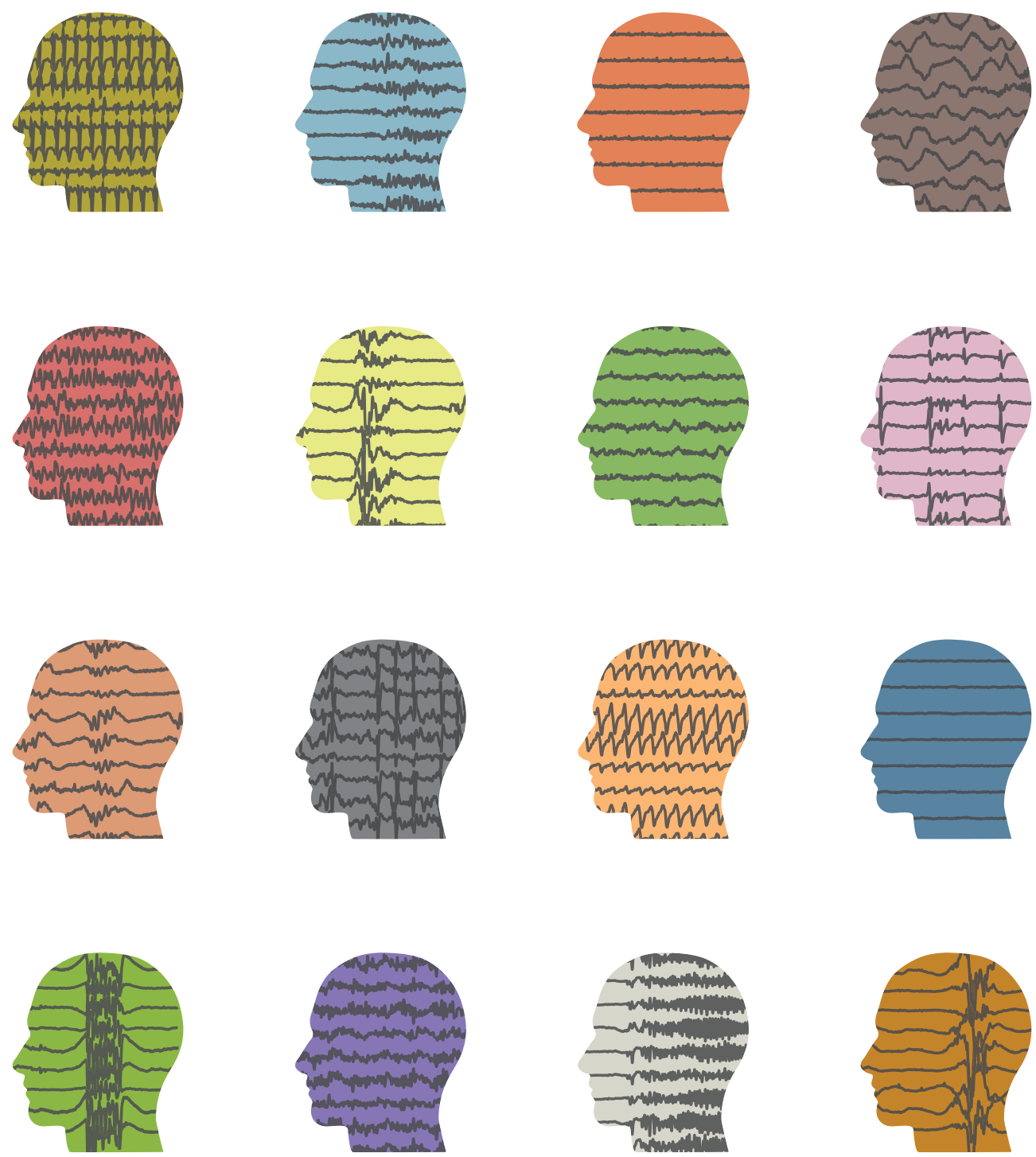

\section{Barry Ruijter}





\section{ELECTROGRAPHIC SIGNATURES OF POSTANOXIC BRAIN INJURY}

Barry Ruijter 


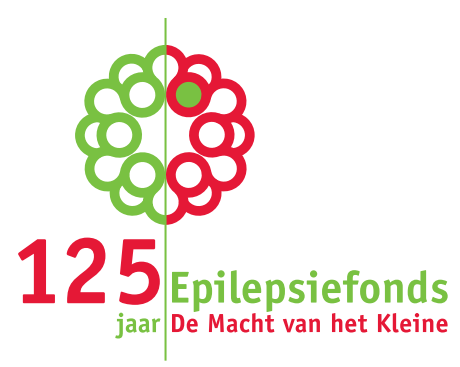

\section{UNIVERSITY | TECHMED OF TWENTE. CENTRE}

The research described in this dissertation was carried out at the department of Clinical Neurophysiology, Technical Medical Centre, University of Twente, the Netherlands. Data were acquired at the departments of Intensive Care of Medisch Spectrum Twente (Enschede), Rijnstate Hospital (Arnhem), St. Antonius Hospital (Nieuwegein), University Medical Center Groningen (Groningen), and VieCuri Medical Center (Venlo).

This work was funded by the Dutch Epilepsy Fund (Epilepsiefonds, grant NEF 14-18).

Printed by: Ipskamp Printing, Enschede

Cover design and layout: Barry Ruijter

The cover figure shows sixteen examples of EEG patterns that were recorded in comatose patients after cardiac arrest. The length of each epoch is approximately 6.5 seconds.

Electrographic signatures of postanoxic brain injury

Barry Ruijter

ISBN: 978-90-365-4605-8

DOI: $10.3990 / 1.9789036546058$

Dutch title: Elektrografische kenmerken van postanoxische hersenschade

Copyright $\odot 2018$ by Barry Ruijter, Amsterdam. All rights reserved. No part of this dissertation may be reproduced or transmitted by print, photocopy, or any other means, without prior written permission of the author. 


\title{
ELECTROGRAPHIC SIGNATURES OF POSTANOXIC BRAIN INJURY
}

\author{
PROEFSCHRIFT
}

\author{
ter verkrijging van \\ de graad van doctor aan de Universiteit Twente, \\ op gezag van de rector magnificus, \\ prof.dr. T.T.M. Palstra, \\ volgens besluit van het College voor Promoties \\ in het openbaar te verdedigen \\ op vrijdag 14 september 2018 om 14:45 uur
}

door

Barry Johannes Ruijter

geboren op 19 september 1985

te Anna Paulowna 
Dit proefschrift is goedgekeurd door:

prof. dr. ir. M.J.A.M. van Putten (promotor)

dr. J. Hofmeijer (co-promotor) 


\section{Samenstelling promotiecommissie}

Voorzitter en secretaris

prof. dr. ir. J.W.M. Hilgenkamp

Universiteit Twente

Promotor

prof. dr. ir. M.J.A.M. van Putten

Universiteit Twente

Co-promotor

dr. J. Hofmeijer

Universiteit Twente

Overige leden

prof. dr. R.J.A. van Wezel

Universiteit Twente

prof. dr. ir. N.J.J. Verdonschot

Universiteit Twente

prof. dr. Y.B.W.E.M. Roos

Universiteit van Amsterdam

dr. J. Horn

prof. dr. J.G van Dijk

Universiteit van Amsterdam

dr. M.B. Westover

Universiteit Leiden

Harvard University, USA 



\section{Contents}

1 General introduction 1

2 Early EEG for outcome prediction of postanoxic coma: a prospective multicenter cohort study

3 The prognostic value of discontinuous EEG patterns in postanoxic coma

4 Generalized epileptiform discharges in postanoxic encephalopathy: quantitative characterization in relation to outcome

5 Propofol does not affect the reliability of early EEG for outcome prediction of comatose patients after cardiac arrest

6 Synaptic damage underlies EEG abnormalities in postanoxic encephalopathy: a computational study

7 Treatment of electrographic status epilepticus after cardiopulmonary resuscitation (TELSTAR): study protocol for a randomized controlled trial

8 General discussion

Summary

Samenvatting

Dankwoord (acknowledgements)

About the author

List of publications 



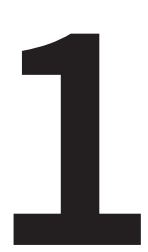

General introduction 
Each year, around 8000 people in the Netherlands are successfully resuscitated after a cardiac arrest ${ }^{1}$. Despite the return of spontaneous circulation, $64-74 \%$ remain comatose upon arrival at the hospital as a result of diffuse postanoxic encephalopathy ${ }^{2,3}$, making cardiac arrest one the most common causes of coma at the intensive care unit ${ }^{4}$. About half of these patients regain consciousness and independence in activities of daily living within six months ${ }^{5,6}$. In the other half, severe anoxic brain damage precludes recovery of consciousness. Most of these patients die, making postanoxic encephalopathy one of the most prevalent causes of death in the hospital.

Treatment options for comatose survivors of a cardiac arrest are limited. Cooling the brain is the only general therapy of presumed benefit, but the gain of this treatment has become unclear since the recent targeted temperature management (TTM) trial ${ }^{6,7}$. Its presumed mechanisms of action include influences on metabolic pathways, inflammation, and apoptosis ${ }^{8}$. Various neuroprotective drugs have been proposed, including corticosteroids, calcium entry blockers, and barbiturates, but none of them proved to be successful in improving outcome ${ }^{9}$. The value of treatment of electrographic or clinical signs of epilepsy, aimed at prevention of secondary neuronal damage, remains unclear ${ }^{10}$.

\subsection{Mechanisms of postanoxic brain injury}

The brain is extremely vulnerable to a depletion in blood supply. Despite comprising only $2 \%$ of the body's weight, it is responsible for approximately $20 \%$ of the total energy consumption ${ }^{11}$. The brain requires a constant supply of glucose and oxygen via the blood stream. Glucose is the major fuel for the nervous system, since fatty acids cannot pass the blood brain barrier. Neurons have no ability to store glucose, and glycogen storage in astrocytes is sufficient to sustain normal brain activity for only seconds ${ }^{12}$. Levels of glucose, oxygen, and ATP fall immediately during cerebral ischemia, and are nearly completely depleted within $10-12$ minutes $^{13}$. After 15 minutes without circulation, up to $95 \%$ of the brain tissue is damaged irreversibly ${ }^{14}$.

Severe or complete interruption of energy supply to neurons is quickly followed by a failure of sodium potassium pumps and an excessive release of glutamate into the extracellular space. This induces anoxic depolarization, with important changes in the intra- and extracellular ion concentrations ${ }^{15}$. Osmosis caused by the ion shifts induces cell swelling, mechanical damage, and necrosis. Massive influx of calcium activates processes that eventually lead to apoptosis. The increased metabolic demand for recovery induces the generation of reactive oxygen species ${ }^{16}$. All these factors contribute to the probability of neural cell death.

In mild to moderate brain ischemia, only synaptic transmission may be affected. Approximately $44 \%$ of the brain's energy consumption is spent on synaptic transmission ${ }^{17}, 2012$ ), which is one of the first processes to fail in case of hypoxia or ischemia ${ }^{18}$. The exacts mechanisms remain unclear, but the available evidence indicates that the initial event is a failure of presynaptic transmitter release ${ }^{19,20}$. Although synaptic failure is initially reversible, isolated synaptic failure may become irreversible ${ }^{21,22}$, associated with persistent failure of presynaptic transmitter release ${ }^{23,24}$. Anoxic long term potentiation of $\mathrm{N}$-methyl-D-aspartate (NMDA)-receptor gated currents 
contributes to exitotoxicity and secondary cell death ${ }^{25}$.

\subsection{Outcome prediction of patients after cardiac arrest}

Early identification of patients without potential for recovery of brain function may prevent inappropriate continuation of medical treatment. Since decisions on treatment withdrawal are irreversible, highly specific tests for the prediction of poor outcome are warranted. Research on these predictors of outcome is vulnerable for the risk of a selffulfilling prophecy ${ }^{26}$. This is the bias occurring when the treating physicians are not blinded to the results of the outcome predictor and use it to make a decision to withdraw life-sustaining treatment.

The introduction of TTM has further complicated outcome predictions of comatose patients after cardiac arrest. Hypothermia and the associated sedatives and muscle relaxants interfere with neurological examination, even after the interruption of sedative drugs, since hypothermia reduces drug clearance ${ }^{27}$.

Most research on outcome prediction after cardiac arrest has focused on prediction of poor outcome. A recent meta-analysis identified bilaterally absent SSEP responses or a combination of absent pupillary light and corneal reflexes plus an $\leq \mathrm{M} 2$ motor response after rewarming as reliable predictors ${ }^{28}$.

For serum biomarkers NSE and S-100B, no consistent threshold for a zero false positive rate could be identified. Myoclonus and status epilepticus were not without false positive predictions, although they both had a strong correlation with poor neurological outcome ${ }^{28}$. A few studies using imaging techniques, including computer tomography and magnetic resonance imaging (MRI), show promising results ${ }^{28}$. However, patient numbers were small and confidence intervals wide. The qualitative nature of these techniques makes them prone to interrater variability. Additionally, measurement times are relatively long and the lack of bedside availability limits the use of these techniques in patients on the intensive care unit ${ }^{28}$.

\subsection{The value of continuous EEG in outcome prediction}

EEG predominantly measures synaptic activity in the cerebral cortex. More specifically, it measures potentials induced by postsynaptic currents on pyramidal cells, which are aligned perpendicular to the skull ${ }^{29}$. Since synaptic transmission is one of the earliest events to fail in cerebral anoxia ${ }^{18}$, and because the cerebral cortex locates most functions that are essential for recovery, EEG is a sensitive tool to monitor the effects of anoxia on the brain. Additional advantages of EEG are its availability at the bedside, its high temporal resolution, and low costs in comparison to other techniques, such as MRI.

The postanoxic EEG evolves as a function of time, on a time scale of many hours ${ }^{30}$. The EEG in first 24 hours after cardiac arrest has been shown to contain most relevant information for the discrimination between patients with good and poor recovery, despite TTM and sedation ${ }^{5,31,32}$. This information can only be captured by appreciating the EEG in relation to time since cardiac arrest. 
Consistently among studies that use early EEG, continuous, normal amplitude patterns, without epileptiform activity at $12 \mathrm{~h}$ after cardiac arrest predict a good outcome ${ }^{33-35}$. Burst-suppression with identical bursts and persisting isoelectric or low voltage EEG patterns $(<20 \mu \mathrm{V})$ at $24 \mathrm{~h}$ after cardiac arrest are reliable predictors of poor outcome ${ }^{5,34}$.

Predictive values of early EEG for outcome of postanoxic coma can be further improved. Categories of EEG patterns used up to now were heterogeneous, suggesting that a refinement could improve discrimination between good and poor recovery. Since postanoxic EEG patterns typically evolve over time, a closer look at their evolution and repeated evaluation could be of additional value above evaluation at a single time point. Furthermore, the use of standardized terminology is needed ${ }^{36,37}$.

\subsection{Computer-assisted analysis of the postanoxic EEG}

The introduction of digital EEG has made interpretation of the EEG more flexible and allows for complex signal processing tasks ${ }^{38}$. The general availability of devices with large data storage capacity has facilitated long time recordings.

Quantitative EEG can be helpful in the assessment of continuous EEG recordings in the ICU. Visual interpretation requires extensive training, is time-consuming, and suffers from interrater variability ${ }^{39,40}$. Otherwise, quantitative analyses are objective, fast, and allow for EEG interpretation by non-experts. Furthermore, quantitative analyses hold the potential to extract more relevant information from the complex EEG signal than visual analysis alone. Various quantitative EEG features have been proposed, including measures for amplitude, continuity, spectral contents, information content, and entropy ${ }^{41}$. The best results for the prediction of outcome after cardiac arrest were achieved by combining features into a single index ${ }^{42}$.

Quantitative measures for EEG background continuity seem to discriminate best between comatose patients after cardiac arrest with good and poor outcome ${ }^{41,43,44}$. However, up to now, these have only been applied to small case series. Features that capture characteristics of burst suppression or epileptiform patterns are promising but lacking.

\section{$\mathbf{1 . 5}$ Influence of sedation on reliability of EEG interpretation}

Previous work shows that differences of EEG patterns between patients with good and poor outcome are largest within the first 24 hours after cardiac arrest ${ }^{5,31,32}$. Consequently, discrimination is optimal within the first $24 \mathrm{~h}$, which coincides with the period of TTM and sedation. Apparently, effects of cerebral ischemia are larger than those of hypothermia and sedation, and predictive values are high, despite treatment.

Effects of hypothermia and sedation on the EEG are well known, and do not include the typical pathological patterns observed after cardiac arrest. Mild therapeutic hypothermia at $33^{\circ} \mathrm{C}$ induces frequency shifts ${ }^{45}$, but the EEG background continuity is preserved with temperatures of $25{ }^{\circ} \mathrm{C}$ and above ${ }^{46}$. Propofol, one of the most commonly used sedative agents in the ICU, can induce burst-suppression or even flattening of the EEG in high doses ${ }^{47}$. However, in the typical doses of $1-3 \mathrm{mg} / \mathrm{kg} / \mathrm{h}$ used in the ICU, the EEG 
remains continuous, with anteriorization of the alpha rhythm ${ }^{48}$. Still, many potential users distrust EEG activity for outcome predictions during these treatments. A quantification of medication effects in postanoxic coma is lacking.

\subsection{Modeling dynamics underlying postanoxic EEG patterns}

Although some microscopic effects of hypoxia and ischemia on the brain are wellknown ${ }^{49}$, their relation with EEG patterns remains unclear. Understanding of the pathophysiology of evolving EEG rhythms after cardiac arrest could support associations between EEG patterns and outcome predictions, and provide opportunities for identification of individualized treatment targets.

Computational models on various scales have been proven useful to improve the understanding of cortical dynamics and the associated macroscopic EEG rhythms ${ }^{50}$. For example, a neural mean field model revealed selective synaptic failure as potential mechanism for GPDs in postanoxic encephalopathy ${ }^{51,52}$. In neural mean field models, individual cell properties and their interactions are replaced by continuous functions that depend on spatial averaging to a certain dergree ${ }^{51}$. This seems reasonable, since a single EEG electrode records currents of well over a 100,000 neurons ${ }^{53}$. Mean field models hold potential to identity mechanisms underlying EEG patterns specific for postanoxic encephalopathy that have remained unexplained, so far.

\subsection{Treatment of electrographic status epilepticus}

In $10-35 \%$ of comatose patients after cardiac arrest EEG patterns are observed that could be classified as 'electrographic status epilepticus' ${ }^{\text {54-56. I }}$. It remains unclear whether this represents a condition to be treated with anti-epileptic drugs to improve outcome, or a sign of severe encephalopathy in which treatment is futile. Evidence for treatment is limited to small, uncontrolled case series, and suggests lack of a relevant effect of treatment ${ }^{10,57}$.

Despite the lack of evidence, most neurologists treat status epilepticus in comatose patients after cardiac arrest with anti-epileptic drugs. Treatment is mostly moderate. Only one third treat these patients equal to those with clinically overt status epilepticus $^{58,59}$. A prospective, randomized clinical trial on the value of intensive anti-epileptic treatment according to status epilepticus guidelines is warranted.

\subsection{Objectives}

The overall objective of the research described in this dissertation is to validate and improve the value of early continuous EEG monitoring for outcome prediction and treatment of comatose patients after a cardiac arrest. Specific objectives are

1. To validate the use of early EEG for the prediction of good and poor outcomes of comatose patients after cardiac arrest.

2. To improve the prognostic value of early EEG by a refinement of categories, standardization of terminology, and determination of optimal timing and duration. 
3. To develop quantitative EEG measures that help to identify patients with a chance of recovery.

4. To quantify the effect of propofol on the postanoxic EEG, and its consequence for the reliability of outcome predictions.

5. To improve the understanding of synaptic mechanisms that underlie characteristic EEG patterns in postanoxic brain injury.

6. To estimate the effect of treatment of electrographic status epilepticus on the outcome of comatose patients after cardiac arrest in a randomized controlled trial.

\subsection{Outline of this dissertation}

In chapter 2, we present a prospective cohort study, conducted on ICUs of five hospitals, on the value of visually assessed EEG at various time points within the first five days after cardiac arrest for prediction of poor or good outcome of comatose patients after cardiac arrest. Chapter 3 describes a quantitative study which investigates the prognostic value of background continuity and amplitude fluctuations. In Chapter 4, we use quantitative EEG to identity patients with possible recovery in the subgroup with epileptiform EEG patterns. In Chapter 5, we investigate whether propofol sedation significantly influences the reliability of qualitative and quantitative measures of EEG for the prediction of outcome after cardiac arrest. In Chapter 6, using a biophysical model, we show that two pathophysiological mechanisms at the synaptic level may explain common EEG patterns in postanoxic encephalopathy. In Chapter 7, we present the study protocol for the ongoing, multicenter, randomized, controlled 'Treatment of ELectroencephalographic STatus epilepticus After cardiopulmonary Resuscitation' (TELSTAR) trial. The general discussion is provided in Chapter 8.

\section{References}

1. Zijlstra JA, Radstok A, Pijls R, Nas J, Beesems SG, Hulleman M, et al. Overleving na een reanimatie buiten het ziekenhuis: vergelijking van de resultaten van 6 verschillende Nederlandse regio's. In: Reanimatie in Nederland. The Hague: Hartstichting; 2016, p. 9-24.

2. Gorjup V, Radsel P, Kocjancic ST, Erzen D, Noc M. Acute ST-elevation myocardial infarction after successful cardiopulmonary resuscitation. Resuscitation 2007; 72: 379-385.

3. Hosmane VR, Mustafa NG, Reddy VK, Reese CL, DiSabatino A, Kolm P, et al. Survival and neurologic recovery in patients with ST-segment elevation myocardial infarction resuscitated from cardiac arrest. Journal of the American College of Cardiology 2009; 53: 409-415.

4. Weiss N, Regard L, Vidal C, Luque Y, Taldir G, Vallet H, et al. Causes of coma and their evolution in the medical intensive care unit. Journal of Neurology 2012; 259: 1474-1477.

5. Hofmeijer J, Beernink TMJ, Bosch FH, Beishuizen A, Tjepkema-Cloostermans MC, Van Putten MJAM. Early EEG contributes to multimodal outcome prediction of postanoxic coma. Neurology 2015; 85: 137-143.

6. Nielsen N, Wetterslev J, Cronberg T, Erlinge D, Gasche Y, Hassager C, et al. Targeted temperature management at $33 \mathrm{C}$ versus $36 \mathrm{C}$ after cardiac arrest. The New England Journal of Medicine 2013; 369: 2197-2206.

7. Bernard S, Gray T. Treatment of comatose survivors of out-of-hospital cardiac arrest with induced hypothermia. The New England Journal of Medicine 2002; 346: 557-563. 
8. González-Ibarra FP, Varon J, López-Meza EG. Therapeutic Hypothermia: Critical Review of the Molecular Mechanisms of Action. Frontiers in Neurology 2011; 2: 4.

9. Wright W, Geocadin R. Postresuscitative Intensive Care: Neuroprotective Strategies after Cardiac Arrest. Seminars in Neurology 2006; 26: 396-402.

10. Hofmeijer J, Tjepkema-Cloostermans MC, Blans MJ, Beishuizen A, van Putten MJAM. Unstandardized Treatment of Electroencephalographic Status Epilepticus Does Not Improve Outcome of Comatose Patients after Cardiac Arrest. Frontiers in Neurology 2014; 5: 39.

11. Attwell D, Laughlin SB. An Energy Budget for Signaling in the Grey Matter of the Brain. Journal of Cerebral Blood Flow \& Metabolism 2001; 21: 1133-1145.

12. Cloutier M, Bolger FB, Lowry JP, Wellstead P. An integrative dynamic model of brain energy metabolism using in vivo neurochemical measurements. Journal of Computational Neuroscience 2009; 27: 391-414.

13. Wagner SR, Lanier WL. Metabolism of Glucose, Glycogen, and High-energy Phosphates during Complete Cerebral Ischemia. Anesthesiology 1994; 81: 1516-1526.

14. Ames A, Wright RL, Kowada M, Thurston JM, Majno G. Cerebral ischemia. II. The no-reflow phenomenon. The American Journal of Pathology 1968; 52: 437-453.

15. Busl KM, Greer DM. Hypoxic-ischemic brain injury: Pathophysiology, neuropathology and mechanisms. NeuroRehabilitation 2010; 26: 5-13.

16. Piantadosi CA, Zhang J. Mitochondrial generation of reactive oxygen species after brain ischemia in the rat. Stroke 1996; 27: 327-332.

17. Howarth C, Gleeson P, Attwell D. Updated energy budgets for neural computation in the neocortex and cerebellum. Journal of Cerebral Blood Flow and Metabolism 2012; 32: 12221232.

18. Hofmeijer J, van Putten MJAM. Ischemic cerebral damage: an appraisal of synaptic failure. Stroke 2012; 43: 607-615.

19. Inagaki N, Seino S. ATP-sensitive potassium channels: structures, functions, and pathophysiology. The Japanese Journal of Physiology 1998; 48: 397-412.

20. Young JN, Somjen GG. Suppression of presynaptic calcium currents by hypoxia in hippocampal tissue slices. Brain Research 1992; 573: 70-76.

21. Hofmeijer J, Mulder ATB, Farinha AC, Van Putten MJAM, Le Feber J. Mild hypoxia affects synaptic connectivity in cultured neuronal networks. Brain Research 2014; 1557: 180-189.

22. Le Feber J, Erkamp N, van Putten MJAM, Hofmeijer J. Loss and recovery of functional connectivity in cultured cortical networks exposed to hypoxia. Journal of Neurophysiology 2017; 118: 394-403.

23. Bolay H, Gursoy-Ozdemir Y, Sara Y, Onur R, Can A, Dalkara T. Persistent Defect in Transmitter Release and Synapsin Phosphorylation in Cerebral Cortex After Transient Moderate Ischemic Injury. Stroke 2002; 33: 1369-1375.

24. Fedorovich S, Hofmeijer J, van Putten MJAM, le Feber J. Reduced Synaptic Vesicle Recycling during Hypoxia in Cultured Cortical Neurons. Frontiers in Cellular Neuroscience 2017; 11: $1-8$.

25. Szatkowski M, Attwell D. Triggering and execution of neuronal death in brain ischaemia: Two phases of glutamate release by different mechanisms. Trends in Neurosciences 1994; 17: 359-365.

26. Geocadin R, Sherman D. Neurological recovery by EEG bursting after resuscitation from cardiac arrest in rats. Resuscitation 2002; 55: 193-200.

27. Leslie K, Sessler DI, Bjorksten AR, Moayeri A. Mild hypothermia alters propofol pharmacokinetics and increases the duration of action of atracurium. Anesthesia and Analgesia 1995; 80: 1007-1014.

28. Sandroni C, Cavallaro F, Callaway CW, D’Arrigo S, Sanna T, Kuiper Ma, et al. Predictors of poor neurological outcome in adult comatose survivors of cardiac arrest: a systematic review and 
meta-analysis. Part 2: Patients treated with therapeutic hypothermia. Resuscitation 2013; 84: 1324-1338.

29. Buzsáki G, Anastassiou CA, Koch C. The origin of extracellular fields and currents - EEG, ECoG, LFP and spikes. Nature Reviews Neuroscience 2012; 13: 407-420.

30. Jørgensen E, Holm S. The natural course of neurological recovery following cardiopulmonary resuscitation. Resuscitation 1998; 36: 111-122.

31. Cloostermans MC, van Meulen FB, Eertman CJ, Hom HW, van Putten MJAM. Continuous electroencephalography monitoring for early prediction of neurological outcome in postanoxic patients after cardiac arrest: A prospective cohort study. Critical Care Medicine 2012; 40: 2867-2875.

32. Oddo M, Rossetti AO. Early multimodal outcome prediction after cardiac arrest in patients treated with hypothermia. Critical Care Medicine 2014; 42: 1340-1347.

33. Hofmeijer J, Tjepkema-Cloostermans MC, van Putten MJAM. Burst-suppression with identical bursts: A distinct EEG pattern with poor outcome in postanoxic coma. Clinical Neurophysiology 2014; 125: 947-954.

34. Sivaraju A, Gilmore EJ, Wira CR, Stevens A, Rampal N, Moeller JJ, et al. Prognostication of post-cardiac arrest coma: early clinical and electroencephalographic predictors of outcome. Intensive Care Medicine 2015; 41: 1264-1272.

35. Spalletti M, Carrai R, Scarpino M, Cossu C, Ammannati A, Ciapetti M, et al. Single electroencephalographic patterns as specific and time-dependent indicators of good and poor outcome after cardiac arrest. Clinical Neurophysiology 2016; 127: 2610-2617.

36. Hirsch LJ, LaRoche SM, Gaspard N, Gerard E, Svoronos A, Herman ST, et al. American Clinical Neurophysiology Society's Standardized Critical Care EEG Terminology. Journal of Clinical Neurophysiology 2013; 30: 1-27.

37. Westhall E, Rosén I, Rossetti AO, van Rootselaar AF, Wesenberg Kjaer T, Friberg H, et al. Interrater variability of EEG interpretation in comatose cardiac arrest patients. Clinical Neurophysiology 2015; 126: 2397-2404.

38. Gavvala J, Abend N, LaRoche S, Hahn C, Herman ST, Claassen J, et al. Continuous EEG monitoring: A survey of neurophysiologists and neurointensivists. Epilepsia 2014; 55: 1864-1871.

39. Swisher CB, Sinha SR. Utilization of Quantitative EEG Trends for Critical Care Continuous EEG Monitoring. Journal of Clinical Neurophysiology 2016; 33: 538-544.

40. Zubler F, Bandarabadi M, Kurmann R, Steimer A, Gast H, Schindler KA. Quantitative EEG in the Intensive Care Unit. Epileptologie 2016; 33: 166-172.

41. Asgari S, Moshirvaziri H, Scalzo F, Ramezan-Arab N. Quantitative measures of EEG for prediction of outcome in cardiac arrest subjects treated with hypothermia: a literature review. Journal of Clinical Monitoring and Computing 2018; p. 1-16.

42. Tjepkema-Cloostermans MC, Hofmeijer J, Beishuizen A, Hom HW, Blans MJ, Bosch FH, et al. Cerebral Recovery Index: Reliable help for prediction of neurologic outcome after cardiac arrest. Critical Care Medicine 2017; 45: 789-797.

43. Noirhomme Q, Lehembre R, del Rosario Lugo Z, Lesenfants D, Luxen A, Laureys S, et al. Automated analysis of background EEG and reactivity during therapeutic hypothermia in comatose patients after cardiac arrest. Clinical EEG and Neuroscience 2014; 45: 6-13.

44. Wennervirta JE, Ermes MJ, Tiainen SM, Salmi TK, Hynninen MS, Särkelä MOK, et al. Hypothermia-treated cardiac arrest patients with good neurological outcome differ early in quantitative variables of EEG suppression and epileptiform activity. Critical Care Medicine 2009; 37: 2427-2435.

45. Kochs E. Electrophysiological monitoring and mild hypothermia. Journal of Neurosurgical Anesthesiology 1995; 7: 222-228.

46. Stecker M, Cheung A, Pochettino A, Kent GP, Patterson T, Weiss SJ, et al. Deep hypothermic circulatory arrest: I. Effects of cooling on electroencephalogram and evoked potentials. 
Annals of Thoracic Surgery 2001; 71: 14-21.

47. San-Juan D, Chiappa KH, Cole AJ. Propofol and the electroencephalogram. Clinical Neurophysiology 2010; 121: 998-1006.

48. Gugino LD, Chabot RJ, Prichep LS, John ER, Formanek V, Aglio LS. Quantitative EEG changes associated with loss and return of consciousness in healthy adult volunteers anaesthetized with propofol or sevoflurane. British Journal of Anaesthesia 2001; 87: 421-428.

49. Martin RL, Lloyd HGE, Cowan Al. The early events of oxygen and glucose deprivation: setting the scene for neuronal death? Trends in Neurosciences 1994; 17: 251-257.

50. Deco G, Jirsa VK, Robinson PA, Breakspear M, Friston K. The Dynamic Brain: From Spiking Neurons to Neural Masses and Cortical Fields. PLoS Computational Biology 2008; 4: e1000092.

51. Liley DTJ, Cadusch PJ, Dafilis MP. A spatially continuous mean field theory of electrocortical activity. Network 2002; 13: 67-113.

52. Tjepkema-Cloostermans MC, Hindriks R, Hofmeijer J, van Putten MJAM. Generalized periodic discharges after acute cerebral ischemia: Reflection of selective synaptic failure? Clinical Neurophysiology 2014; 125: 255-262.

53. Niedermeyer E, Lopes da Silva F. Niedermeyer's Electroencephalography: Basic Principles, Clinical Applications, and Related Fields. Philadelphia: Lippincott Williams \& Wilkins; 2004.

54. Legriel S, Bruneel F, Sediri H, Hilly J, Abbosh N, Lagarrigue MH, et al. Early EEG monitoring for detecting postanoxic status epilepticus during therapeutic hypothermia: a pilot study. Neurocritical Care 2009; 11: 338-344.

55. Rittenberger JC, Popescu A, Brenner RP, Guyette FX, Callaway CW. Frequency and timing of nonconvulsive status epilepticus in comatose post-cardiac arrest subjects treated with hypothermia. Neurocritical Care 2012; 16: 114-122.

56. Rossetti AO, Oddo M, Liaudet L, Kaplan PW. Predictors of awakening from postanoxic status epilepticus after therapeutic hypothermia. Neurology 2009; 72: 744-749.

57. Bouwes A, Van Rootselaar AF, Biemond-Moeniralam HS, Teunissen LL, Tromp SC, Hijdra A, et al. Status epilepticus after cardiopulmonary resuscitation: a case cohort study. In: Postanoxic coma: prognosis after therapeutic hypothermia (PhD thesis); 2012, p. 117-129.

58. Abend NS, Dlugos DJ, Hahn CD, Hirsch LJ, Herman ST. Use of EEG monitoring and management of non-convulsive seizures in critically ill patients: a survey of neurologists. Neurocritical Care 2010; 12: 382-389.

59. Bouwes A, Kuiper MA, Hijdra A, Horn J. Induced hypothermia and determination of neurological outcome after CPR in ICUs in the Netherlands: results of a survey. Resuscitation 2010; 81: 393-397. 



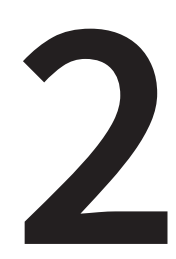

\section{Early EEG for outcome prediction of postanoxic coma: a} prospective multicenter cohort study

Submitted as: B.J. Ruijter, M.C. Tjepkema-Cloostermans, S.C. Tromp, W.M. van den Bergh, N.A. Foudraine, F.H.M. Kornips, G. Drost, E. Scholten, F.H. Bosch, A. Beishuizen, M.J.A.M. van Putten, J. Hofmeijer, Early EEG for outcome prediction of postanoxic coma: a prospective multicenter cohort study. 


\section{Abstract}

Background Recent work indicates that early EEG contributes to outcome prediction of postanoxic coma. We aim to validate these findings and align with international EEG terminology to provide high quality evidence that EEG after cardiac arrest allows for reliable prediction of good or poor outcome.

Methods

Findings

Consecutive, comatose survivors after cardiac arrest were included in a prospective cohort study conducted in five centers. Continuous EEG recordings were started as soon as possible and continued up to five days. Five-minute EEG epochs were assessed by two reviewers, independently, at eight predefined time points from $6 \mathrm{~h}$ to 5 days after cardiac arrest, blinded for patients 'actual condition, treatment, and outcome. EEGpatterns were categorized as generalized suppression $(<10 \mu \mathrm{V})$, synchronous patterns with $\geq 50 \%$ suppression, continuous, or other. Outcome at six months was categorized as good (Cerebral Performance Category 1-2) or poor (CPC 3-5).

We included 850 patients, of which $46 \%$ had a good outcome. Generalized suppression and synchronous patterns with $\geq 50 \%$ suppression predicted poor outcome without false positives at 6 h after cardiac arrest or later. Their summed sensitivity was 0.47 (95\% confidence interval $(\mathrm{Cl}): 0.42-0.51)$ at $12 \mathrm{~h}$ and $0.30(95 \%-\mathrm{Cl}$ : $0.26-0.33)$ at $24 \mathrm{~h}$ after cardiac arrest, with specificity $1.00(95 \%-$ $\mathrm{Cl}$ : 0.99-1.00) at both time points. At $36 \mathrm{~h}$ or later, sensitivity for poor outcome was $\leq 0.22$. Continuous EEG-patterns at $12 \mathrm{~h}$ predicted good outcome with sensitivity of 0.50 (95\%-Cl: $0.46-0.55)$ and specificity of 0.91 (95\%-Cl: $0.88-0.93)$; at $24 \mathrm{~h}$ or later, specificity for the prediction of good outcome was $<0.90$. Reliability of predictions was equal among centers, despite different treatment regimes.

Interpretation Generalized suppression and synchronous patterns with $\geq 50 \%$ suppression allow for reliable prediction of poor outcome in the first five days after cardiac arrest, with maximum sensitivity in the first 24 hours. Continuous EEG patterns at $12 \mathrm{~h}$ after cardiac arrest have a strong association with good recovery. 


\subsection{Introduction}

Postanoxic brain injury is among the most frequent causes of coma in the Intensive Care Unit (ICU). The chance of recovery of consciousness and independence in activities of daily living within six months is approximately $50 \%{ }^{1,2}$. Early prediction of recovery perspectives may guide decisions on continuation or withdrawal of life sustaining treatment. Current guidelines focus on prediction of poor outcome and recommend the use of absent pupillary light and corneal reflexes or bilaterally absent somatosensory evoked potential (SSEP) responses for decisions on treatment withdrawal, given their low false-positive rates ${ }^{3,4}$. However, of these predictors, sensitivity to identification of patients with a poor outcome is limited, ranging from 13 to $48 \%$, and their reliability is insufficient during hypothermia and sedation ${ }^{5}$.

Recent studies have shown that the EEG contains valuable information to assist in prediction of outcome after cardiac arrest. This information could only be extracted when appreciating EEG patterns in relation to the time since cardiac arrest. The best discrimination between patients with good and poor outcomes was possible with EEG recorded within the first $24 \mathrm{~h}$ after cardiac arrest, despite treatment with targeted temperature management (TTM) and sedation ${ }^{2,6}$. The prognostic value of the EEG seemed lower after the first $24 \mathrm{~h}$ and remains unclear for the period beyond $72 \mathrm{~h}^{2,7-11}$. Previous indiscriminate studies did not acknowledge the time-dependency of EEG patterns ${ }^{1,12-15}$.

In all studies on early EEG for prognostication after cardiac arrest, a continuous, normal amplitude background pattern at $12 \mathrm{~h}$ was strongly associated with a good neurological outcome ${ }^{2,8,10}$. Otherwise, isoelectric or low-voltage patterns at $24 \mathrm{~h}$ after cardiac arrest were invariably associated with poor outcome ${ }^{2,8}$. Time-independent predictors of poor outcome were generalized period discharges on a suppressed background ${ }^{8,11,16}$, and burst-suppression with identical bursts ${ }^{2,8,10}$. Results on the prognostic value of other burst-suppression patterns are conflicting ${ }^{2,8-11}$.

With this study, we validate the use of early EEG for outcome prediction of coma after cardiac arrest in a multicenter prospective cohort study. In order to improve predictive values and applicability, we use recent findings to refine EEG categories ${ }^{2}$ and align classification with standardized critical care EEG terminology ${ }^{17}$. We determine optimal timing and assess the additional yield of EEG recordings beyond $24 \mathrm{~h}$.

\subsection{Methods}

\subsubsection{Study design and participants}

This is a prospective cohort study on intensive care units of five teaching hospitals in the Netherlands (Medisch Spectrum Twente, Rijnstate Hospital, St. Antonius Hospital, University Medical Center Groningen, and VieCuri Medical Centre). Consecutive, adult, comatose (Glasgow Coma Scale $<8$ or suspected in sedated patients) patients after cardiac arrest were included. EEG recordings were started as soon as possible after admission, preferably within $12 \mathrm{~h}$ after cardiac arrest. For practical reasons, EEG recordings were only started between 8 A.M. and 8 P.M. in each center, and not during weekend in one center. EEG recordings were continued until patients were awake or died, with a maximum of five days. Part of the EEG data from two centers were used in previous publications on visual or quantitative EEG analyses ${ }^{2,18,19}$. In the participating 
hospitals, continuous EEG monitoring was considered standard care for patients after cardiac arrest. The Medical Research Ethics Committee Twente waived the need for informed consent for the EEG monitoring. Informed consent was obtained from surviving patients at time of follow up.

\subsubsection{Procedures}

Patients were treated according to standard protocols for comatose patients after cardiac arrest. A target temperature of $33^{\circ} \mathrm{C}$ or $36^{\circ} \mathrm{C}$ was induced as soon as possible after arrival on the ICU and maintained for $24 \mathrm{~h}$. Patients received propofol, midazolam, or both for sedation, and morphine, fentanyl, or remifentanil for analgesia. In one center, the majority of patients was anesthetized with sevoflurane instead of propofol or midazolam.

Withdrawal of treatment was considered $\geq 72 \mathrm{~h}$ after cardiac arrest, during normothermia and off sedation. Decisions on treatment withdrawal were based on international guidelines including bilateral absence of the somatosensory evoked potential (SSEP), absent or extensor motor responses, and absent of brainstem reflexes ${ }^{3,4}$. Decisions on treatment withdrawal were sporadically taken between 48 and $72 \mathrm{~h}$ in case of absent brain stem reflexes or SSEP responses. EEG data were not used for decisions regarding treatment withdrawal. However, physicians were not blinded to the EEG in order to allow early detection and treatment of electrographic seizure activity.

Continuous EEG recordings were started as soon as possible after arrival at the ICU and continued up to five days, or until discharge from the ICU. Twenty-one electrodes were placed on the scalp according to the international 10-20 system. Visual analysis of EEG data was pre-specified and performed offline, after the recordings. A computer algorithm selected 5 minute artifact-free EEG epochs at 6, 12, 24, 36, 48, 72, 96, and $120 \mathrm{~h}$ after cardiac arrest to be presented to a reviewer ${ }^{2}$. If no epoch was available at these time points, because of artifacts, the closest available artifact-free epoch in the range $\pm 2 \mathrm{~h}$ was used. Before visual assessment, signals were band pass filtered (range: 0.5 to $35 \mathrm{~Hz}$ ). Visual assessment was performed using a longitudinal bipolar montage. EEG epochs were presented in random order to reviewers who were blinded to the timing of the epoch, the clinical condition of the patients, medication, and outcome. All EEG epochs were assessed by two experienced reviewers from a pool of six (B.R., M.T-C, M.v.P., H.K., A.G., or J.H.), independently. If the two reviewers disagreed, the final classification was determined by consensus. If necessary, a third reviewer was consulted. Reviewers were allowed to choose the option "No classification possible" if the epoch was considered unreliable due to artifacts.

EEG categorization was based on previous work ${ }^{2,8,11}$, with definitions updated and aligned with the ACNS standardized critical care EEG terminology to allow for better reproducibility ${ }^{17}$. EEG patterns were classified as generalized suppression (all activity $<10 \mu \mathrm{V}$ ), synchronous patterns with $\geq 50 \%$ suppression (generalized periodic discharges on a suppressed background, or burst-suppression with generalized, abrupt onset bursts, with suppressed background and at least 50\% of time spent in suppression), continuous (continuous or nearly continuous patterns without periodic activity), or other. Burst-suppression with identical bursts ${ }^{20}$, and highly epileptiform bursts typically fulfilled the criteria for 'synchronous burst-suppression'. Spatially 

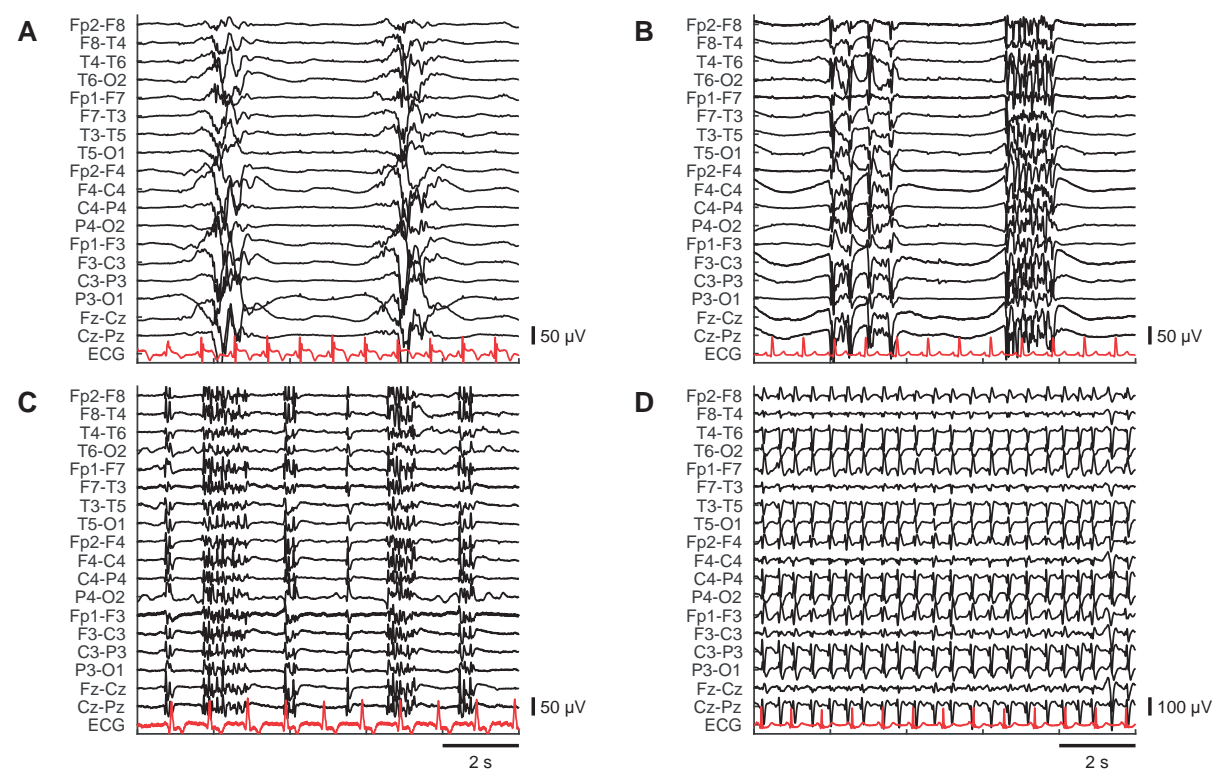

Figure 2.1: Examples of synchronous patterns with $\geq 50 \%$ suppression. A: burst suppression with identical bursts. B: burst-suppression with abrupt onset, generalized bursts (these bursts could alternatively be described as "highly epileptiform bursts"). C: burst-suppression with abrupt onset, generalized bursts, alternated with generalized discharges. D: generalized periodic discharges on a suppressed background.

heterogeneous burst-suppression patterns were classified as 'other patterns'. Continuous patterns were subdivided according to their dominant frequency (delta, theta, or $\geq$ alpha). See Figure 2.1 for examples of synchronous patterns with $\geq 50 \%$ suppression.

Additionally collected data included age, sex, resuscitation details, and maximum doses of sedative medication.

\subsubsection{Outcome}

The primary outcome measure was neurological functional recovery at six months, expressed as the score on the five-point Glasgow-Pittsburgh Cerebral Performance Category (CPC) ${ }^{21}$, dichotomized as good (CPC 1 or 2) or poor (CPC 3, 4, or 5). Outcome was assessed during a standardized telephone interview by one of two investigators (BR or MT-C) or a trained research nurse. CPC scores were based on a Dutch translation of the EuroQol-6D questionnaire. In one center, CPC scores were assessed using the Short Form 36 (SF-36) questionnaire.

\subsubsection{Statistical analysis}

In order to compare patients with good and poor outcomes, categorical variables were analyzed using Pearson's $\chi^{2}$-test, continuous variables using the Mann-Whitney test. Interrater reliability (IRR) for the categorization of EEG patterns was tested using Cohen's Kappa. Sensitivity and specificity were calculated for EEG predictors of poor or good outcome, including corresponding 95\% confidence intervals. P-values 
$<0.05$ were considered statistically significant. All tests were performed using Matlab Statistics Toolbox software (MATLAB and Statistics Toolbox Release R2016b, The MathWorks, Inc., Natick, Massachusetts, United States).

\subsection{Results}

Between May 2010 and November 2017, EEG recordings were started in 887 comatose patients after cardiac arrest. Fourteen had no artifact-free EEG at any of the investigated time points and 23 were lost to follow-up, leaving 850 patients for the analyses. We visually assessed a total number of 3232 EEG epochs. Categorization was impossible for 139 epochs (4\%) due to artifacts.

Clinical characteristics are shown in Table 2.1, grouped by outcome. Poor outcome occurred in 455 patients (54\%). As expected, patients with poor outcome were older, more often had a non-cardiac cause of arrest, and less often had ventricular fibrillation (VF) as initial rhythm. Patients with a good outcome required higher doses of sedation and analgesia. EEG recordings were stopped earlier in patients with a good outcome (52 vs. $62 \mathrm{~h}$ after cardiac arrest), because recordings were terminated at awakening.

Generalized EEG suppression (all activity $<10 \mu \mathrm{V}$ ) and synchronous patterns with $\geq 50 \%$ suppression were invariably associated with a poor outcome, from six hours after cardiac arrest onwards (Figure 2.2). Sensitivity for detection of patients with a poor outcome reached its maximum at $12 \mathrm{~h}(0.47,95 \%-\mathrm{Cl}: 0.42-0.51)$ and gradually decreased thereafter (Figure 2.3A, Table 2.2). Specificity was $100 \%$ in all participating centers, despite different treatment regimens, and sensitivity ranged from 0.13 to 0.55 (Table 2.2). It should be noted that the center with the lowest sensitivity had only 13 patients with an EEG epoch available at $12 \mathrm{~h}$.

Continuous EEG patterns were strongly associated with a good outcome, if present within $12 \mathrm{~h}$ after cardiac arrest. At $12 \mathrm{~h}$, sensitivity was $0.50(95 \%-\mathrm{Cl}: 0.46-0.55)$ at specificity of 0.91 (95\%-Cl: $0.88-0.93)$. At later time points, sensitivity increased even further, but at the cost of a lower specificity (Figure 2.3B). Specificity of a continuous EEG pattern for prediction of good outcome was not different among participating centers, whereas sensitivity ranged from 0.46 to 0.88 (Table 2 ).

With an intermediate EEG pattern, the chance of a good outcome was time-dependent. This was most striking for discontinuous patterns (Figure 2.2): the chance of a good outcome decreased gradually from $80 \%$ at $6 \mathrm{~h}$ to $0 \%$ at $120 \mathrm{~h}$. Likewise, the chance of a good outcome of heterogeneous burst-suppression (i.e. not classified as 'synchronous pattern with $\geq 50 \%$ suppression') decreased from $37 \%$ at $12 \mathrm{~h}$ to $0 \%$ at $72 \mathrm{~h}$ and later. All patients with an epileptiform EEG pattern within the first 24 hours, or a low voltage EEG at 48 hours or later, had a poor outcome.

The chance to identify a poor outcome was highest if EEG recordings were started within $12 \mathrm{~h}$ after cardiac arrest. For subjects with poor outcome that had their first EEG evaluated at $12 \mathrm{~h}$, the probability of reliable identification of poor outcome was $55 \%$. With cEEG starting between $12 \mathrm{~h}$ and $24 \mathrm{~h}$, this probability was $36 \%(\mathrm{p}<0.001)$, and with start time $>24$ h only $24 \%(p<0.001)$. 
Table 2.1: Patient characteristics, grouped by outcome.

\begin{tabular}{lccc}
\hline & $\begin{array}{c}\text { Poor outcome } \\
(\text { CPC 3-5) }\end{array}$ & $\begin{array}{c}\text { Good outcome } \\
(\text { CPC 1-2) }\end{array}$ & P-value \\
\hline Number & $455(54 \%)$ & $395(46 \%)$ & \\
Age & $67(57-75)$ & $60(51-69)$ & $<0.001$ \\
Female & $121(27 \%)$ & $84(21 \%)$ & 0.07 \\
Out-of-hospital cardiac arrest & $407(89 \%)$ & $367(93 \%)$ & 0.08 \\
Noncardiac cause of arrest & $94(24 \%)$ & $21(6 \%)$ & $<0.001$ \\
VF as initial cardiac rhythm & $248(58 \%)$ & $352(91 \%)$ & $<0.001$ \\
Therapeutic hypothermia $\left(33^{\circ} \mathrm{C}\right)$ & $214(47 \%)$ & $179(45 \%)$ & 0.62 \\
EEG start time $(\mathrm{h}$ after cardiac arrest) & $11(6-19)$ & $11(6-19)$ & 0.70 \\
EEG stop time $(\mathrm{h}$ after cardiac arrest) & $62(42-93)$ & $52(41-78)$ & 0.01 \\
Treatment with propofol & $378(85 \%)$ & $352(91 \%)$ & 0.01 \\
$\quad$ Max. dose in first 24h $(\mathrm{mg} / \mathrm{kg} / \mathrm{h})$ & $2.7(2.0-3.6)$ & $3.1(2.3-3.9)$ & $<0.001$ \\
Treatment with midazolam & $132(30 \%)$ & $105(27 \%)$ & 0.43 \\
$\quad$ Max. dose in first 24h $(\mu \mathrm{gg} / \mathrm{kg} / \mathrm{h})$ & $99(57-162)$ & $96(67-164)$ & 0.77 \\
Treatment with fentanyl & $204(48 \%)$ & $155(40 \%)$ & 0.10 \\
$\quad$ Max. dose in first $24 \mathrm{~h}(\mu \mathrm{gg} / \mathrm{kg} / \mathrm{h})$ & $1.3(1.0-1.8)$ & $1.5(1.2-2.2)$ & 0.002 \\
Treatment with remifentanil & $33(7 \%)$ & $21(5 \%)$ & 0.25 \\
$\quad$ Max. dose in first 24h $(\mu \mathrm{gg} / \mathrm{kg} / \mathrm{h})$ & $3.6(2.5-5.6)$ & $6.6(3.3-11.4)$ & 0.02 \\
Treatment with morphine & $174(39 \%)$ & $192(50 \%)$ & $<0.001$ \\
$\quad$ Max. dose in first 24h $(\mu \mathrm{kg} / \mathrm{kg} / \mathrm{h})$ & $25(22-31)$ & $25(21-29)$ & 0.26 \\
Treatment with sevoflurane & $30(7 \%)$ & $21(5 \%)$ & 0.43 \\
$\quad$ Max. end-tidal volume $\%$ & $1.2(1.1-1.4)$ & $1.4(1.2-1.6)$ & 0.03 \\
SSEP performed & $276(61 \%)$ & $43(11 \%)$ & $<0.001$ \\
$\quad$ N20 bilaterally absent & $123(27 \%)$ & $0(0 \%)$ & $<0.001$ \\
\hline
\end{tabular}

Data are shown as number (percentage) or median (interquartile range). SSEP: somatosensory evoked potential, VF: ventricular fibrillation. 
Time after cardiac arrest

\begin{tabular}{|c|c|c|c|c|c|c|c|c|c|}
\hline & & $\begin{array}{c}6 \mathrm{~h} \\
(\mathrm{~N}=340)\end{array}$ & $\begin{array}{c}12 \mathrm{~h} \\
(\mathrm{~N}=469)\end{array}$ & $\begin{array}{c}24 \mathrm{~h} \\
(\mathrm{~N}=742)\end{array}$ & $\begin{array}{c}36 \mathrm{~h} \\
(\mathrm{~N}=673)\end{array}$ & $\begin{array}{c}48 \mathrm{~h} \\
(\mathrm{~N}=517)\end{array}$ & $\begin{array}{c}72 \mathrm{~h} \\
(\mathrm{~N}=298)\end{array}$ & $\begin{array}{c}96 \mathrm{~h} \\
(\mathrm{~N}=133)\end{array}$ & $\begin{array}{c}120 \mathrm{~h} \\
(\mathrm{~N}=60)\end{array}$ \\
\hline 윽 & Suppression & $\begin{array}{l}0 \% \\
(0-2)\end{array}$ & $\begin{array}{c}0 \% \\
(0-1)\end{array}$ & $\begin{array}{c}0 \% \\
(0-1)\end{array}$ & $\begin{array}{c}0 \% \\
(0-1)\end{array}$ & $\begin{array}{l}0 \% \\
(0-1)\end{array}$ & $\begin{array}{c}0 \% \\
(0-2)\end{array}$ & & \\
\hline \multirow{2}{*}{ 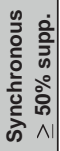 } & BS (synchronous) & $\begin{array}{l}0 \% \\
(0-2)\end{array}$ & $\begin{array}{c}0 \% \\
(0-1)\end{array}$ & $\begin{array}{l}0 \% \\
(0-1)\end{array}$ & $\begin{array}{l}0 \% \\
(0-1)\end{array}$ & $\begin{array}{l}0 \% \\
(0-1)\end{array}$ & $\begin{array}{l}0 \% \\
(0-2)\end{array}$ & $\begin{array}{l}0 \% \\
(0-3)\end{array}$ & \\
\hline & GPDs (supp. bg.) & & $\begin{array}{l}0 \% \\
(0-1)\end{array}$ & $\begin{array}{c}0 \% \\
(0-1)\end{array}$ & $\begin{array}{c}0 \% \\
(0-1)\end{array}$ & $\begin{array}{c}0 \% \\
(0-1)\end{array}$ & $\begin{array}{l}0 \% \\
(0-2)\end{array}$ & $\begin{array}{c}0 \% \\
(0-3)\end{array}$ & $\begin{array}{l}0 \% \\
(0-6)\end{array}$ \\
\hline \multirow{3}{*}{ 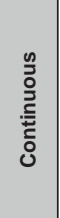 } & Continuous (delta) & $\begin{array}{c}67 \% \\
(61-72)\end{array}$ & $\begin{array}{c}82 \% \\
(77-86)\end{array}$ & $\begin{array}{c}50 \% \\
(46-54)\end{array}$ & $\begin{array}{c}47 \% \\
(42-51)\end{array}$ & $\begin{array}{c}56 \% \\
(51-61)\end{array}$ & $\begin{array}{c}58 \% \\
(52-64)\end{array}$ & $\begin{array}{c}48 \% \\
(39-58)\end{array}$ & $\begin{array}{c}50 \% \\
(36-64)\end{array}$ \\
\hline & Continuous (theta) & $\begin{array}{c}82 \% \\
(77-87)\end{array}$ & $\begin{array}{c}79 \% \\
(74-83)\end{array}$ & $\begin{array}{c}77 \% \\
(73-80)\end{array}$ & $\begin{array}{c}71 \% \\
(66-74)\end{array}$ & $\begin{array}{c}64 \% \\
(59-69)\end{array}$ & $\begin{array}{c}59 \% \\
(53-65)\end{array}$ & $\begin{array}{c}38 \% \\
(30-48)\end{array}$ & $\begin{array}{l}13 \% \\
(5-24)\end{array}$ \\
\hline & Continuous ( $\geq$ alpha) & $\begin{array}{l}82 \% \\
(77-87)\end{array}$ & $\begin{array}{c}91 \% \\
(87-94)\end{array}$ & $\begin{array}{c}92 \% \\
(89-94)\end{array}$ & $\begin{array}{l}93 \% \\
(90-95)\end{array}$ & $\begin{array}{l}79 \% \\
(74-82)\end{array}$ & $\begin{array}{c}78 \% \\
(72-83)\end{array}$ & $\begin{array}{l}75 \% \\
(66-82)\end{array}$ & \\
\hline \multirow{4}{*}{ 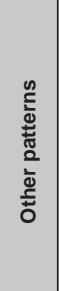 } & Low-voltage & $\begin{array}{c}19 \% \\
(14-24)\end{array}$ & $\begin{array}{c}16 \% \\
(13-20)\end{array}$ & $\begin{array}{l}0 \% \\
(0-1)\end{array}$ & $\begin{array}{c}10 \% \\
(7-13)\end{array}$ & $\begin{array}{l}0 \% \\
(0-1)\end{array}$ & $\begin{array}{l}0 \% \\
(0-2)\end{array}$ & $\begin{array}{l}0 \% \\
(0-3)\end{array}$ & $\begin{array}{l}0 \% \\
(0-6)\end{array}$ \\
\hline & Epileptiform (other) & $\begin{array}{l}0 \% \\
(0-2)\end{array}$ & $\begin{array}{l}0 \% \\
(0-1)\end{array}$ & $\begin{array}{c}13 \% \\
(10-16)\end{array}$ & $\begin{array}{c}16 \% \\
(13-20)\end{array}$ & $\begin{array}{c}14 \% \\
(11-18)\end{array}$ & $\begin{array}{c}18 \% \\
(13-23)\end{array}$ & $\begin{array}{c}26 \% \\
(18-35)\end{array}$ & $\begin{array}{c}25 \% \\
(15-38)\end{array}$ \\
\hline & BS (heterogeneous) & $\begin{array}{c}30 \% \\
(25-36)\end{array}$ & $\begin{array}{c}37 \% \\
(32-42)\end{array}$ & $\begin{array}{c}13 \% \\
(10-16)\end{array}$ & $\begin{array}{c}25 \% \\
(21-29)\end{array}$ & $\begin{array}{c}20 \% \\
(16-24)\end{array}$ & $\begin{array}{l}0 \% \\
(0-2)\end{array}$ & $\begin{array}{c}0 \% \\
(0-3)\end{array}$ & $\begin{array}{l}0 \% \\
(0-6)\end{array}$ \\
\hline & Discontinuous & $\begin{array}{c}80 \% \\
(75-85)\end{array}$ & $\begin{array}{c}70 \% \\
(66-75)\end{array}$ & $\begin{array}{c}46 \% \\
(42-50)\end{array}$ & $\begin{array}{c}30 \% \\
(26-34)\end{array}$ & $\begin{array}{c}25 \% \\
(21-29)\end{array}$ & $\begin{array}{c}35 \% \\
(30-42)\end{array}$ & $\begin{array}{l}14 \% \\
(8-21)\end{array}$ & $\begin{array}{l}0 \% \\
(0-6)\end{array}$ \\
\hline
\end{tabular}

Figure 2.2: EEG-patterns and their time-dependent positive predictive value (PPV) for good outcome. Each cell shows the PPV (95\% confidence interval) for a combination of EEG pattern and timing relative to the cardiac arrest BG: background pattern, BS: burst-suppression, GPDs: generalized periodic discharges, Supp: suppression. 


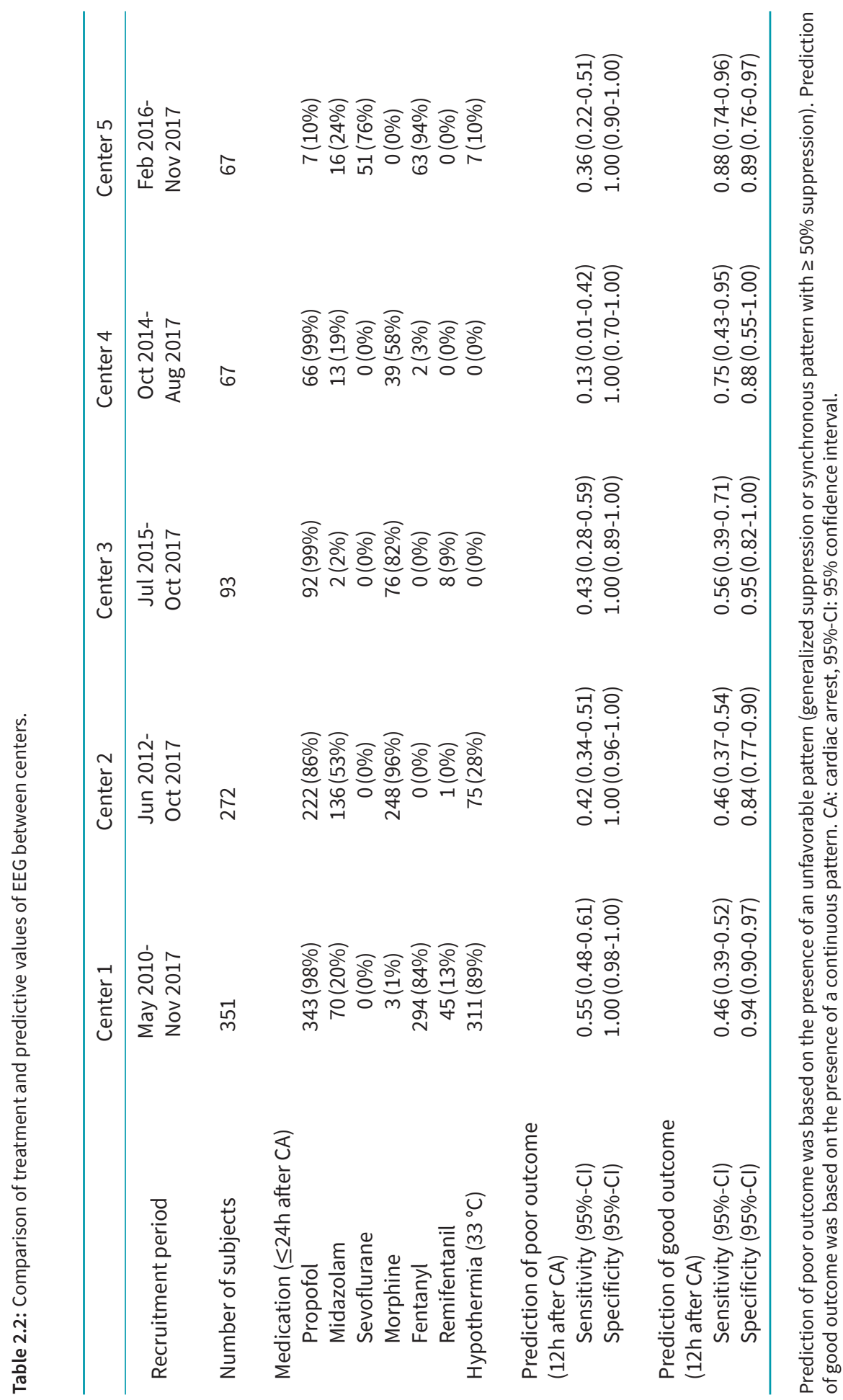



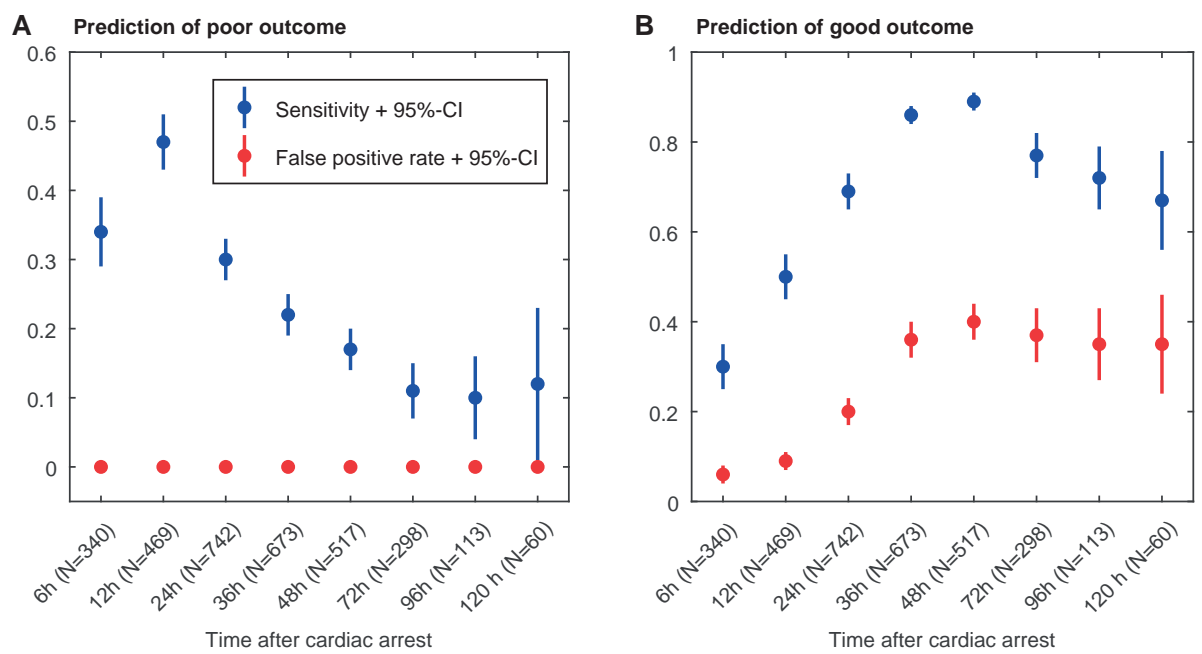

Figure 2.3: Predictive value of the EEG as a function of time after cardiac arrest. A: test characteristics for the prediction of poor outcome based on 'suppression' or 'synchronous pattern with $\geq 50 \%$ suppression'. B: test characteristics for the prediction of good outcome based on 'continuous' EEG pattern. Error bars indicate the $95 \%$ confidence intervals. Numbers $(\mathrm{N})$ refer to the total number of patients with an EEG epoch available at the indicated time point.

With repeated EEG evaluation, the proportion of patients in whom reliable prediction of outcome was possible increased (Figure 4). Having at least one unfavorable EEG ('suppression' or 'synchronous pattern with $\geq 50 \%$ suppression') at 6,12 , or $24 \mathrm{~h}$ after cardiac arrest yielded a sensitivity of 0.52 (95\%-Cl: $0.47-0.58)$ at specificity of 1.00 (95\%-Cl: 0.98-1.00). By including the information obtained between $36 \mathrm{~h}$ and 5 days after cardiac arrest, prediction of poor outcome improved only marginally. Sensitivity for good outcome improved by assessment of the EEG at more than one point in time. Since the proportion of patients with continuous EEG patterns and poor outcome also increased over time, this was at the cost of specificity. The presence of at least one continuous EEG pattern at $6 \mathrm{~h}$ or $12 \mathrm{~h}$ yielded a sensitivity of $63 \%$ (95\%-Cl: $57-68)$, at specificity of $90 \%$ (95\%-Cl: $86-93)$. The cumulative sensitivity for prediction of good outcome at $120 \mathrm{~h}$ was $98 \%$ (95\%-Cl: 96-99), at a specificity of $69 \%$ (95\%-Cl: $64-74)$. None of the patients with a continuous EEG at $12 \mathrm{~h}$ showed 'suppression' or 'synchronous pattern with $\geq 50 \%$ suppression' throughout the remainder of the EEG recording.

At $12 \mathrm{~h}$ after cardiac arrest, the interrater reliability was 0.80 (95\%-Cl: $0.74-0.86)$ for discrimination between continuous and other patterns and 0.78 (95\%-Cl: 0.72-0.85) for discrimination between unfavorable ('suppression' or 'synchronous pattern with $\geq 50 \%$ suppression') and other patterns. The IRR was 0.85 (95\%-Cl: $0.77-0.92)$ for the distinction between 'synchronous burst-suppression' and other burst-suppression patterns.

In combination, SSEP and early EEG identified more patients with a poor outcome than EEG alone. Of those with EEG available within the first $24 \mathrm{~h}$ after cardiac arrest, an unfavorable pattern ('suppression' or 'synchronous pattern with $\geq 50 \%$ suppression') at 6 , 
A

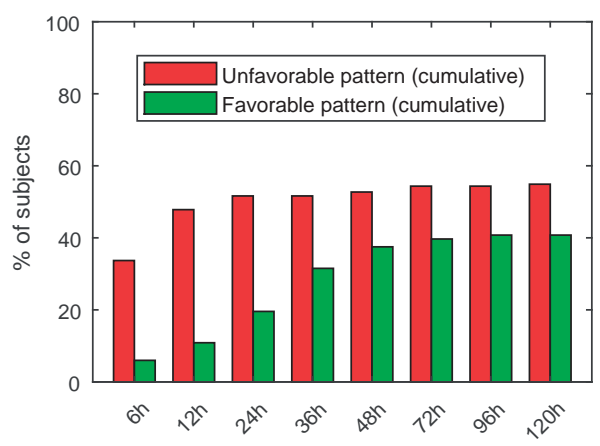

B Patients with good outcome ( $\mathrm{N}=155)$

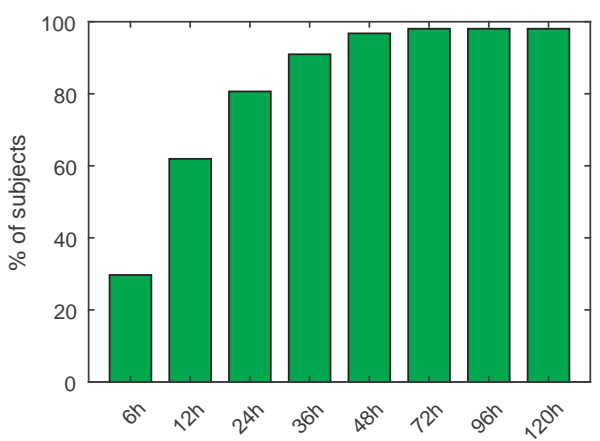

Figure 2.4: Prognostic yield of repeated EEG assessment. This analysis includes only patients with an EEG recording started within 6 hours after cardiac arrest. Red bars indicate the fraction of subjects in which an unfavorable EEG pattern ('suppression' or a 'synchronous pattern with $\geq 50 \%$ suppression') was observed up to the indicated time point. Green bars indicate the fraction of subjects in which a favorable ('continuous') EEG pattern was observed up to the indicated time point. A: results for all 185 patients with poor outcome. B: results for all 155 patients with good outcome.

12 , or $24 \mathrm{~h}$ identified 181 of 420 (43\%) patients with a poor outcome. In the same group, absent SSEP responses allowed for reliable prediction of outcome in an additional 31 patients (7\%).

\subsection{Discussion}

With this prospective cohort study, including nearly 900 patients from five hospitals, we provide high quality evidence confirming that early EEG allows reliable prediction of outcome of comatose patients after cardiac arrest. Generalized suppression or 'synchronous patterns with at least $50 \%$ suppression' were invariably associated with a poor outcome between $6 \mathrm{~h}$ and 5 days after cardiac arrest. A continuous background pattern at 6 or $12 \mathrm{~h}$ was strongly associated with a good recovery. Predictive values were highest at $12-24 \mathrm{~h}$ after cardiac arrest. Predictors were equally specific among five centers, despite differences in treatment regimes. We confirm that unfavorable EEG patterns and absent SSEP responses have complementary value for the prediction of poor outcome.

Our results validate previous findings on reliability and time dependency of EEG patterns ${ }^{2}$. The achieved improvement of sensitivity for reliable prediction of poor outcome, from 0.29 to 0.47 , was achieved by lumping previously identified unfavorable EEG categories ${ }^{2,8,18}$, and by aligning definitions with standardized terminology ${ }^{17}$. Studies reporting higher sensitivities were either retrospective, inheriting the risk of selection bias ${ }^{8,10}$, or not without false positives ${ }^{1}$. Studies showing conflicting results did not account for time dependency ${ }^{5}$. In line with international terminology ${ }^{17}$, we now used a suppressed background pattern (indicating $<10 \mu \mathrm{V}$ ) as hallmark. The previously reported low voltage criterion (indicating $<20 \mu \mathrm{V}$ ) EEG was not $100 \%$ specific for the prediction of poor outcome, since two patients with a low voltage patterns at $36 \mathrm{~h}$ eventually recovered. One group reported a few cases that recovered 
despite a suppressed EEG at 12 or $24 \mathrm{~h}$ after cardiac arrest, but in their definition recovery of consciousness was sufficient for 'good outcome' ${ }^{10}$.

We show that repeated assessment of the EEG within the first $24 \mathrm{~h}$ after cardiac arrest improves the sensitivity for reliable detection of either good or poor outcome. These results contradict findings of a smaller study, which concluded that continuous EEG does not have additional value over routine spot EEGs during hypothermia ${ }^{22}$. However, this previous work did not account for evolution of the EEG during the first $24 \mathrm{~h}$ after cardiac arrest. With the current study, the prognostic yield of prolonging continuous EEG beyond $24 \mathrm{~h}$ was limited. However, diagnosis of epileptiform patterns, which might warrant treatment, was not taken into account.

We confirm the reliability for prediction of poor outcome 'synchronous patterns with $\geq 50 \%$ suppression'. One of its subgroups is burst-suppression with abrupt onset, generalized bursts on a suppressed background, with at least $50 \%$ of the record consisting of suppression. Sixty-five percent of these patterns showed identical bursts ${ }^{20}$. The second subgroup generalized periodic discharges on a suppressed background ${ }^{8,11}$. These results are in line with findings of our recent quantitative analysis, in which we showed that an amplitude ratio between non-suppressed and suppressed segments of $\geq 6.12$ is invariably associated with a poor outcome ${ }^{23}$.Some authors have claimed that all burst-suppression patterns predicts a poor outcome, regardless of the burst type ${ }^{8,9}$, 11,24 . This was typically with studies starting $>72 \mathrm{~h}$ after cardiac arrest ${ }^{9,11}$. One study that included burst-suppression as predictor of poor outcome in early EEG, and did not specify burst-types, was not without false positives ${ }^{1}$. Our definition of 'synchronous burst-suppression' seems robust, since interrater agreement for the distinction of 'synchronous burst-suppression' and other burst-suppression patterns was good.

We only investigated spontaneous EEG patterns and did not assess background reactivity of the EEG. The presence of reactivity seems very sensitive for prediction of a good outcome, but lacks specificity to make relevant predictions of outcome ${ }^{1}$. Results on absent reactivity for the prediction of poor outcome are conflicting ${ }^{1,8,10,11,25}$, most likely resulting from a lack of standardization of stimulus protocols and quantitative definitions of reactivity ${ }^{26}$. Studies combing analysis of background EEG pattern and reactivity for prediction of outcome after cardiac arrest are lacking.

EEG interpretation in this study may have been influenced by the use of sedative medication. However, typical doses used were much lower than those needed to induce burst-suppression in healthy brains ${ }^{27,28}$. Additionally, quantitative studies suggest that the effects of sedation on the EEG are small as compared to the effects of the anoxic encephalopathy ${ }^{23,29}$.

Although this study meets the Standards for the Reporting of Diagnostic accuracy studies (STARD) criteria (www.stard-statement.org), it has limitations. Like most studies on prognostication of comatose patients after cardiac arrest, we cannot exclude the potential bias of self-fulfilling prophecy ${ }^{30}$. To minimize this risk, decisions on treatment withdrawal were based on international guidelines including bilaterally absent SSEP, absent or extensor motor responses, and absent brain stem reflexes ${ }^{3,4}$. The EEG within the first $72 \mathrm{~h}$ after cardiac arrest was not taken into account. 
Second, outcome for some of the patients may have been influenced by causes unrelated to the postanoxic encephalopathy. Because we aimed for a realistic patient sample, not biased by selection, we did not exclude patients that died from other organ failure, such as a second cardiac arrest. This may have limited the specificity of our predictions of good outcome. Finally, visual assessment of EEG is subject to interrater variability. Nevertheless, the interrater reliability for the distinction between 'generalized suppression or synchronous patterns with $>50 \%$ suppression' and 'continuous EEG' from other patterns was good (IRR=0.78-0.85), and probably better than those reported for absent SSEP responses (IRR=0.2-0.76) ${ }^{31,32}$.

\section{References}

1. Rossetti AO, Tovar Quiroga DF, Juan E, Novy J, White RD, Ben-Hamouda N, et al. Electroencephalography Predicts Poor and Good Outcomes After Cardiac Arrest. Critical Care Medicine 2017; 45: e674-e682.

2. Sondag L, Ruijter BJ, Tjepkema-Cloostermans MC, Beishuizen A, Bosch FH, van Til JA, et al. Early EEG for outcome prediction of postanoxic coma: prospective cohort study with costminimization analysis. Critical Care 2017; 21: 111.

3. Sandroni C, Cariou A, Cavallaro F, Cronberg T, Friberg H, Hoedemaekers C, et al. Prognostication in comatose survivors of cardiac arrest: An advisory statement from the European Resuscitation Council and the European Society of Intensive Care Medicine. Resuscitation 2014; 85: 1779-1789.

4. Wijdicks EFM, Hijdra A, Young GB, Bassetti CL, Wiebe S. Practice parameter: prediction of outcome in comatose survivors after cardiopulmonary resuscitation (an evidence-based review): report of the Quality Standards Subcommittee of the American Academy of Neurology. Neurology 2006; 67: 203-210.

5. Sandroni C, Cavallaro F, Callaway CW, D’Arrigo S, Sanna T, Kuiper Ma, et al. Predictors of poor neurological outcome in adult comatose survivors of cardiac arrest: a systematic review and meta-analysis. Part 2: Patients treated with therapeutic hypothermia. Resuscitation 2013; 84: 1324-1338.

6. Oddo M, Rossetti AO. Early multimodal outcome prediction after cardiac arrest in patients treated with hypothermia. Critical Care Medicine 2014; 42: 1340-1347.

7. Fugate JE, Wijdicks EFM, Mandrekar J, Claassen DO, Manno EM, White RD, et al. Predictors of neurologic outcome in hypothermia after cardiac arrest. Annals of Neurology 2010; 68: 907-914.

8. Sivaraju A, Gilmore EJ, Wira CR, Stevens A, Rampal N, Moeller JJ, et al. Prognostication of post-cardiac arrest coma: early clinical and electroencephalographic predictors of outcome. Intensive Care Medicine 2015; 41: 1264-1272.

9. Søholm H, Kjær TW, Kjaergaard J, Cronberg T, Bro-Jeppesen J, Lippert FK, et al. Prognostic value of electroencephalography (EEG) after out-of-hospital cardiac arrest in successfully resuscitated patients used in daily clinical practice. Resuscitation 2014; 85: 1580-1585.

10. Spalletti M, Carrai R, Scarpino M, Cossu C, Ammannati A, Ciapetti M, et al. Single electroencephalographic patterns as specific and time-dependent indicators of good and poor outcome after cardiac arrest. Clinical Neurophysiology 2016; 127: 2610-2617.

11. Westhall E, Rossetti AO, van Rootselaar AF, Wesenberg Kjaer T, Horn J, Ullén S, et al. Standardized EEG interpretation accurately predicts prognosis after cardiac arrest. Neurology 2016; 86: 1482-1490.

12. Amorim E, Rittenberger JC, Zheng JJ, Westover MB, Baldwin ME, Callaway CW, et al. Continuous EEG monitoring enhances multimodal outcome prediction in hypoxic-ischemic brain injury. Resuscitation 2016; 109: 121-126. 
13. Crepeau AZ, Rabinstein AA, Fugate JE, Mandrekar J, Wijdicks EF, White RD, et al. Continuous EEG in therapeutic hypothermia after cardiac arrest: Prognostic and clinical value. Neurology 2013; 80: 339-344.

14. Rundgren M, Westhall E, Cronberg T, Rosén I, Friberg H. Continuous amplitude-integrated electroencephalogram predicts outcome in hypothermia-treated cardiac arrest patients. Critical Care Medicine 2010; 38: 1838-1844.

15. Youn CS, Callaway CW, Rittenberger JC. Combination of initial neurologic examination, quantitative brain imaging and electroencephalography to predict outcome after cardiac arrest. Resuscitation 2017; 110: 120-125.

16. Ruijter BJ, van Putten MJAM, Hofmeijer J. Generalized epileptiform discharges in postanoxic encephalopathy: Quantitative characterization in relation to outcome. Epilepsia 2015; 56 : $1845-1854$.

17. Hirsch LJ, LaRoche SM, Gaspard N, Gerard E, Svoronos A, Herman ST, et al. American Clinical Neurophysiology Society's Standardized Critical Care EEG Terminology. Journal of Clinical Neurophysiology 2013; 30: 1-27.

18. Ruijter BJ, Hofmeijer J, Tjepkema-Cloostermans MC, van Putten MJAM. The prognostic value of discontinuous EEG patterns in postanoxic coma. Clinical Neurophysiology 2018; 129 : 1534-1543.

19. Tjepkema-Cloostermans MC, Hofmeijer J, Beishuizen A, Hom HW, Blans MJ, Bosch FH, et al. Cerebral Recovery Index: Reliable help for prediction of neurologic outcome after cardiac arrest. Critical Care Medicine 2017; 45: 789-797.

20. Hofmeijer J, Tjepkema-Cloostermans MC, van Putten MJAM. Burst-suppression with identical bursts: A distinct EEG pattern with poor outcome in postanoxic coma. Clinical Neurophysiology 2014; 125: 947-954.

21. Jennett $B$, Bond M. Assessment of outcome after severe brain damage: A practical scale. The Lancet 1975; 305: 480-484.

22. Alvarez V, Sierra-Marcos A, Oddo M, Rossetti AO. Yield of intermittent versus continuous EEG in comatose survivors of cardiac arrest treated with hypothermia. Critical Care 2013; 17: R190.

23. Ruijter BJ, Hofmeijer J, Tjepkema-Cloostermans MC, van Putten MJAM. The prognostic value of discontinuous EEG patterns in postanoxic coma. Clinical Neurophysiology 2018; 129: 1534-1543.

24. Lamartine Monteiro M, Taccone FS, Depondt C, Lamanna I, Gaspard N, Ligot N, et al. The Prognostic Value of 48-h Continuous EEG During Therapeutic Hypothermia After Cardiac Arrest. Neurocritical Care 2016; 24: 153-162.

25. Amorim E, Rittenberger JC, Zheng JJ, Westover MB, Baldwin ME, Callaway CW, et al. Continuous EEG monitoring enhances multimodal outcome prediction in hypoxic-ischemic brain injury HHS Public Access. Resuscitation 2016; 109: 121-126.

26. Admiraal MM, van Rootselaar AF, Horn J. Electroencephalographic reactivity testing in unconscious patients: a systematic review of methods and definitions. European Journal of Neurology 2017; 24: 245-254.

27. Huotari AM, Koskinen M, Suominen K, Alahuhta S, Remes R, Hartikainen KM, et al. Evoked EEG patterns during burst suppression with propofol. British Journal of Anaesthesia 2004; 92: 18-24.

28. Scheller MS, Nakakimura K, Fleischer JE, Zornow MH. Cerebral effects of sevoflurane in the dog: Comparison with isoflurane and enflurane. British Journal of Anaesthesia 1990; 65: 388-392.

29. Drohan CM, Cardi Al, Rittenberger JC, Popescu A, Callaway CW, Baldwin ME, et al. Effect of sedation on quantitative electroencephalography after cardiac arrest. Resuscitation 2018; 124: 132-137. 
30. Geocadin RG, Peberdy MA, Lazar RM. Poor survival after cardiac arrest resuscitation. Critical Care Medicine 2012; 40: 979-980.

31. Pfeifer R, Weitzel S, Günther A, Berrouschot J, Fischer M, Isenmann S, et al. Investigation of the inter-observer variability effect on the prognostic value of somatosensory evoked potentials of the median nerve (SSEP) in cardiac arrest survivors using an SSEP classification. Resuscitation 2013; 84: 1375-1381.

32. Zandbergen EGJ, Hijdra A, de Haan RJ, van Dijk JG, Ongerboer de Visser BW, Spaans F, et al. Interobserver variation in the interpretation of SSEPs in anoxic-ischaemic coma. Clinical Neurophysiology 2006; 117: 1529-1535. 



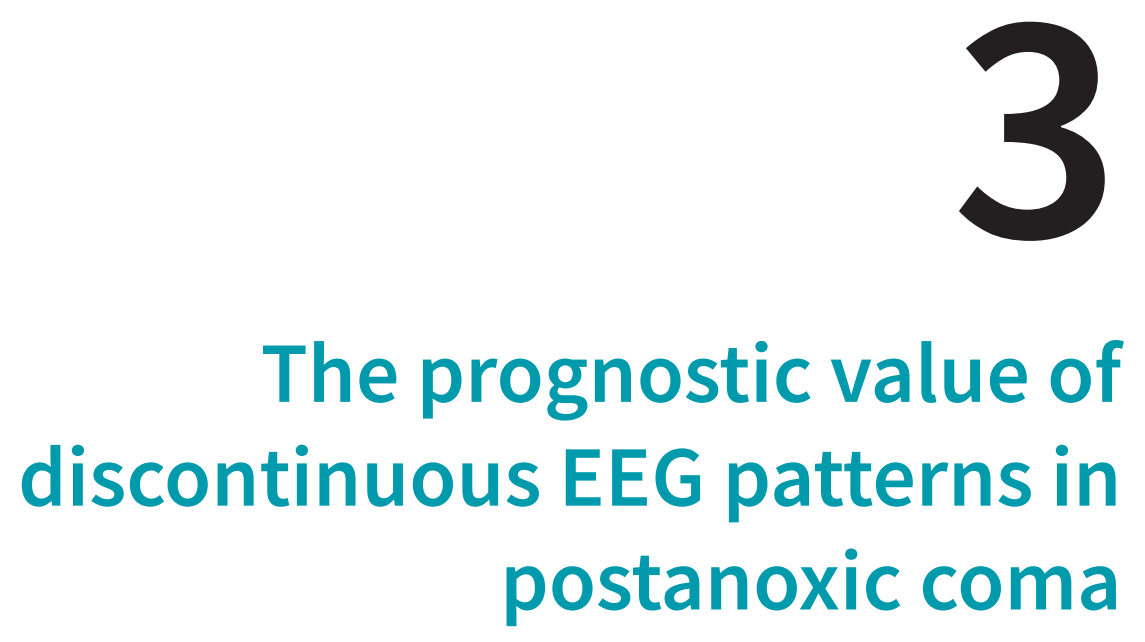

Published as: B.J. Ruijter, J. Hofmeijer, M.C. Tjepkema-Cloostermans, M.J.A.M. van Putten, The prognostic value of discontinuous EEG patterns in postanoxic coma, Clinical Neurophysiology 2018; 129: 1534-1543. 


\section{Abstract}

Objective

Methods

Results

Conclusions

Significance
To assess the value of background continuity and amplitude fluctuations of the EEG for the prediction of outcome of comatose patients after cardiac arrest.

In a prospective cohort study, we analyzed EEGs recorded in the first 72 hours after cardiac arrest. We defined the background continuity index $(\mathrm{BCl})$ as the fraction of EEG not spent in suppressions (amplitudes $<10 \mu \mathrm{V}$ for $\geq 0.5 \mathrm{~s}$ ), and the burst-suppression amplitude ratio (BSAR) as the mean amplitude ratio between non-suppressed and suppressed segments. Outcome was assessed at 6 months and categorized as "good" (Cerebral Performance Category 1-2) or "poor" (CPC 3-5).

Of the 559 patients included, $46 \%$ had a good outcome. Combinations of $\mathrm{BCl}$ and $\mathrm{BSAR}$ resulted in the highest prognostic accuracies. Good outcome could be predicted at 24 hours with $57 \%$ sensitivity (95\% confidence interval (Cl): 48-67) at 90\% specificity (95\%-Cl: 86-95). Poor outcome could be predicted at 12 hours with $50 \%$ sensitivity $(95 \%-\mathrm{Cl}: 42-56)$ at $100 \%$ specificity $(95 \%-\mathrm{Cl}$ : 99-100).

EEG background continuity and the amplitude ratio between bursts and suppressions reliably predict the outcome of postanoxic coma.

The presented features provide an objective, rapid, and reliable tool to assist in EEG interpretation in the Intensive Care Unit. 


\subsection{Introduction}

Early outcome prediction of comatose patients after cardiac arrest remains a challenge. The EEG is a sensitive and reliable tool, especially within the first 24 hours after cardiac arrest ${ }^{1}$. A timely restoration of continuous, normal amplitude background activity is essential for good neurological recovery. Consistently among various studies, a continuous EEG pattern within 12 hours after cardiac arrest predicts a good outcome, whereas a persistent isoelectric or low-voltage EEG at 24 hours predicts a poor outcome ${ }^{2-4}$.

In addition to the EEG background continuity, the degree of amplitude fluctuation seems to be a powerful indicator of the severity of postanoxic encephalopathy. EEG patterns with pronounced alternations in amplitudes, such as burst-suppression with identical bursts ${ }^{5}$, and generalized periodic discharges (GPDs) on a suppressed background $^{6}$, have a strong association with poor outcome.

Although both background continuity and amplitude fluctuations play an important role in the visual assessment of the postanoxic EEG, they remain hard to quantify by a human observer. Continuity and relative amplitude are usually treated as categorical variables ${ }^{7}$, for which interrater agreement is far from perfect ${ }^{8,9}$. Therefore, visual analysis alone has probably not been able to elucidate the discriminative power of these features.

The assessment of discontinuous EEG patterns can be improved by the use of quantitative EEG (qEEG). Quantitative measures do not suffer from interrater variability, can be applied by non-experts, and save reviewing time ${ }^{10-12}$. Quantitative measures for EEG background continuity have proven to be valuable for the prediction of outcome in postanoxic coma, but have only been applied to small case series ${ }^{13,14}$ or selected EEG patterns ${ }^{6}$, so far. The prognostic value of the amplitude ratio between bursts and suppressions has, to the best of our knowledge, never been investigated in any visual or quantitative analysis.

In this study, we relate outcome of postanoxic coma to quantitative measures of EEG background continuity and the amplitude ratio between bursts and suppressions. We will show that these straightforward measures are reliable predictors of outcome, with sensitivities higher than reported for visual EEG analysis. Since the presented features remain closely related to key visual observations, they will also provide insights to be of value in routine EEG assessment.

\subsection{Methods}

\subsubsection{Patients}

This study was designed as a prospective cohort study on continuous EEG monitoring for outcome prediction of comatose patients after cardiac arrest. Data were collected between June 2010 and April 2017 in Medisch Spectrum Twente and Rijnstate, two large teaching hospitals in the Netherlands. All consecutive adult patients that were admitted comatose after cardiac arrest to the Intensive Care Unit (ICU) were included. Parts of the EEG data up to December 2015 were used previously for studies on outcome prediction after cardiac arrest using visual ${ }^{15-17}$ or quantitative analysis ${ }^{11,18}$. The Medical Research Ethics Committee Twente waived the need for informed consent for EEG monitoring during the ICU stay and clinical follow-up. 


\subsubsection{Standard of care}

Patients were treated according to standard protocols for comatose patients after cardiac arrest. A target temperature of $33^{\circ} \mathrm{C}$ or $36^{\circ} \mathrm{C}$ was induced as soon as possible after arrival on the ICU and maintained for 24 hours. In Medisch Spectrum Twente, propofol and either fentanyl or remifentanil were used for sedation and analgesia. In Rijnstate hospital, patients received a combination of propofol, midazolam, and morphine. Continuous EEG recordings were started as soon as possible after admission to the ICU, and for practical reasons always between 8 AM and 8 PM. Twenty-one silver/silver chloride cup electrodes were placed on the scalp according to the international 10-20 system. EEG recordings were continued until patients were awake or until the decision to withdraw treatment was made, with a maximum of five days.

\subsubsection{Decisions on withdrawal of care}

Withdrawal of treatment was considered $\geq 72$ hours after cardiac arrest, during normothermia and off sedation. Decisions on treatment withdrawal were based on international guidelines including bilateral absence of the somatosensory evoked potential (SSEP), absent or extensor motor responses, and incomplete return of brainstem reflexes ${ }^{19,20}$. Decisions on treatment withdrawal were sporadically taken between 48 and 72 hours in case of absent SSEP responses. EEG data were not used for decisions regarding treatment withdrawal. However, physicians were not blinded to the EEG and treatment of electrographic seizures was left to the discretion of the treating physician.

\subsubsection{Outcome}

We considered outcome good if patients were alive and independent in activities of daily living at six months. Therefore, the primary outcome measure was neurological outcome expressed as the score on the five-point Glasgow-Pittsburgh Cerebral Performance Category (CPC) ${ }^{21}$, dichotomized between good (CPC 1 or 2) and poor (CPC 3, 4, or 5). Outcome was assessed during a standardized telephone interview at six months by one of two investigators (BR or MT-C). CPC scores were based on a Dutch translation of the EuroQol-6D questionnaire.

\subsubsection{Clinical characteristics}

Additionally collected clinical data included age, gender, resuscitation details, and maximum doses of sedative medication. These characteristics were compared between patients with good and poor outcomes. Categorical variables were analyzed using Pearson's $\chi^{2}$-test, continuous variables using the Mann-Whitney test. P-values $<0.05$ were considered statistically significant. All tests were performed using Matlab Statistics Toolbox software (MATLAB and Statistics Toolbox Release R2016b, The MathWorks, Inc., Natick, Massachusetts, United States).

\subsubsection{EEG preprocessing and artifact rejection}

Analysis of the EEG data was performed offline. All routines were implemented in Matlab (MATLAB Release R2016b, The MathWorks, Inc.). Only EEG recorded in the first 72 hours after cardiac arrest was used for the analysis. Epochs were created by selecting the first five minutes of each consecutive hour of the recording, with timing relative to the cardiac arrest. Before any analysis, the EEG was transformed to the longitudinal 
Table 3.1: Definition of artifact parameters and threshold values for exclusion.

\begin{tabular}{|c|c|c|c|}
\hline $\begin{array}{l}\text { Type of } \\
\text { artifacts }\end{array}$ & Preprocessing steps & Parameter definition & $\begin{array}{l}\text { Threshold } \\
\text { value }\end{array}$ \\
\hline $\begin{array}{l}\text { Muscle } \\
\text { artifacts }\end{array}$ & $\begin{array}{l}\text { Calculation of } \\
\text { power spectral den- } \\
\text { sity using Welch's } \\
\text { averaged peri- } \\
\text { odogram. }\end{array}$ & $\begin{array}{l}\text { Mean power in high frequency band } \\
(25-40 \mathrm{~Hz}) \text { divided by mean power in } \\
\text { low frequency band }(4-12 \mathrm{~Hz}) \text {. Only } \\
\text { calculated if mean power in high } \\
\text { frequency band }>1 \mu \mathrm{V}^{2} \text {. }\end{array}$ & $>0.5$ \\
\hline $\begin{array}{l}\text { High- } \\
\text { amplitude } \\
\text { peaks } \\
\text { (absolute) }\end{array}$ & $\begin{array}{l}\text { Butterworth high } \\
\text { pass filter (cutoff } \\
\text { frequency: } 0.5 \mathrm{~Hz} \text { ) }\end{array}$ & Peak amplitude in 5-min epoch & $\begin{array}{l}>1000 \mu \mathrm{V} \\
\text { or } \leq 1000 \mu \mathrm{V} \\
\text { with simulta- } \\
\text { neous ampli- } \\
\text { tude }>1000 \\
\mu \mathrm{V} \text { in other } \\
\text { channel }\end{array}$ \\
\hline $\begin{array}{l}\text { High- } \\
\text { amplitude } \\
\text { peaks } \\
\text { (relative) }\end{array}$ & $\begin{array}{l}\text { Butterworth high } \\
\text { pass filter (cutoff } \\
\text { frequency: } 0.5 \mathrm{~Hz} \text { ), } \\
\text { followed by mov- } \\
\text { ing average filter } \\
\text { with window length } \\
\text { 1s over absolute } \\
\text { values. }\end{array}$ & $\begin{array}{l}\text { Maximum ratio between amplitude } \\
\text { and mean amplitude of other chan- } \\
\text { nels. Only calculated if amplitude } \\
\text { was } \geq 20 \mu \mathrm{V} \text { and after excluding } \\
\text { channels based on parameter AMP } 1 \text {. }\end{array}$ & $>5$ \\
\hline $\begin{array}{l}\text { Flat } \\
\text { channels }\end{array}$ & $\begin{array}{l}\text { Unfiltered EEG } \\
\text { divided into one- } \\
\text { second segments. }\end{array}$ & $\begin{array}{l}\text { Fraction of segments with standard } \\
\text { deviation smaller than } 0.1 \mu \mathrm{V} \text {. }\end{array}$ & $>0.01$ \\
\hline
\end{tabular}

bipolar montage. Subsequently, an artifact detection algorithm was applied to reject three types of artifacts: flat channels, muscle artifacts, and high-amplitude artifacts resulting from head movements or loose electrodes. Definitions of the artifact parameters are given in Table 3.1. Artifact detection was performed per channel. Channels were excluded if at least one of the artifact parameters exceeded a threshold value. If more than 6 of the 18 recording channels were excluded by the artifact rejection algorithm, the epoch was considered unreliable and not used for further analysis. Thresholds for the artifact parameters had been determined after testing them on all available epochs of 50 randomly selected patients. Epochs and channels that passed the artifact rejection algorithm were filtered using a sixth order zero-phase Butterworth bandpass filter (frequency range: $0.5-30 \mathrm{~Hz}$ ).

\subsubsection{Definition of quantitative EEG (qEEG) features}

'Suppressions' were defined as segments with amplitudes $<10 \mu \mathrm{V}$ for at least $500 \mathrm{~ms}^{6}$. The amplitude threshold value is based on the commonly used definition of a suppressed EEG background ${ }^{7}$. Since standard definitions for the minimum duration of suppressions are lacking, we chose $500 \mathrm{~ms}$ as the best compromise between the need 
to detect suppressions in between GPDs and the need to avoid the classification of spontaneous fluctuations in amplitude as suppressions. The background continuity index $(\mathrm{BCl})$ was defined as the fraction of EEG not spent in suppressions, and could take values between 0 and 1 .

The burst-suppression amplitude ratio (BSAR) was subsequently defined as the standard deviation of signal outside suppressions divided by the standard deviation of signal within suppressions, resulting in BSAR $\geq 1$. The BSAR was calculated only if 0.01 $\leq \mathrm{BCl} \leq 0.99$, otherwise, $B S A R=1$. Since high-amplitude artifacts can have a large influence on the BSAR, all epochs with BSAR $\geq 4$ were checked visually on any remaining artifacts and excluded if necessary. Features were calculated per channel, after which mean values were used for further analysis.

\subsubsection{Influence of sedative medication}

Based on their pharmacological properties, we expected potential effects of propofol and midazolam on our qEEG features. Propofol is well known to induce burstsuppression patterns at higher doses (San-Juan et al., 2010). Midazolam reduces the EEG amplitude ${ }^{22}$ and may also induce burst-suppression patterns in critically ill patients ${ }^{23}$. These effects may decrease the $\mathrm{BCl}$ and increase the BSAR, and therefore lead to falsely pessimistic predictions of the outcome of patients under sedation.

To investigate the effects of propofol, midazolam, and other sedative medication used, we performed a linear regression analysis relating their maximum doses in the first 24 hours to the extracted qEEG parameters in the same period. To give each patient equal weight in the regression analysis, individual data points were weighted by the total number of data points included for the corresponding patient.

\subsubsection{Evaluation of value of qEEG features for outcome prediction}

The predictive values of the quantitative measures were evaluated for any of the first 72 hours after cardiac arrest. If for some patient a particular hour was unavailable, we used the closest available value in the range \pm 2 hours. Receiver Operating Characteristic $(\mathrm{ROC})$ curves were plotted to determine the optimal threshold values for prediction of either good or poor outcome. For the prediction of good outcome, we allowed for a maximum of $10 \%$ false-positives. For the prediction of poor outcome, we only considered threshold values without false-positive predictions in our data set.

\subsubsection{Prediction model}

To investigate the joint value of $\mathrm{BCl}$ and $\mathrm{BSAR}$ as outcome predictors of postanoxic coma, we defined the following prediction model:

$$
\mathrm{P}_{\text {good }}=\frac{\mathrm{BCl}}{1+\exp \left(k \cdot\left(\mathrm{BSAR}_{-} \mathrm{BSAR}_{0}\right)\right)},
$$

where $\mathrm{P}_{\text {good }}$ represents the chance of a good outcome. The model coefficients $B_{S A R_{0}}$ and $k$ were obtained by fitting model predictions to the binary outcome measure. In the prediction model, the chance of a good outcome linearly increases with the $\mathrm{BCl}$, yielding maximum values for continuous EEG patterns $(\mathrm{BCl}=1)$ and zero for suppressed EEG $(\mathrm{BCl}=0)$. The chance of a good outcome decreases exponentially for increasing 
BSAR values. The coefficient $\mathrm{BSAR}_{0}$ represents the value for which the maximally achievable $\mathrm{P}_{\text {good }}$ is 0.5 , and $k$ determines how rapidly $\mathrm{P}_{\text {good }}$ approaches zero for BSAR values above $\mathrm{BSAR}_{0}$. Since we expected a strong time-dependency of the coefficients, a separate model was constructed for each of the first 72 hours after cardiac arrest.

We validated the models using 10 -fold cross-validation. In this procedure, datasets were partitioned into 10 equal subsets, each called a 'fold'. Subsequently, one fold was used for validation and the remaining data for fitting the model. By repeating this procedure ten times, each entry was used for validation once. Test accuracies were calculated by averaging accuracies for each of the ten folds, and corresponding 95\% confidence intervals by using bootstrap estimates ( $N=2000)$.

\subsection{Results}

\subsubsection{Patients}

EEG recordings were started in 582 patients. Twenty-three patients were lost to followup, leaving 559 patients for the analysis. Outcome was good in 46\%. Baseline characteristics are listed in Table 3.2 and were as expected: patients with good outcome were younger ( 60 vs. 68 years, $p<0.001$ ), more often had a primary cardiac cause of the arrest $(94 \%$ vs. $76 \%, p<0.001)$, ventricular fibrillation as initial rhythm ( $92 \%$ vs. $58 \%$, $\mathrm{p}<0.001$ ), and required higher doses of propofol ( $2.95 \mathrm{vs} .2 .67 \mathrm{mg} / \mathrm{kg} / \mathrm{h}, \mathrm{p}=0.03$ ), fentanyl ( 1.72 vs. $1.38 \mu \mathrm{g} / \mathrm{kg} / \mathrm{h}, \mathrm{p}=0.002)$, and remifentanil (6.67 vs. $3.23 \mu \mathrm{g} / \mathrm{kg} / \mathrm{h}, \mathrm{p}=0.01$ ) for sedation.

\subsubsection{Artifact rejection}

Thresholds for the artifact rejection algorithm were based on visual inspection of the algorithm performance in a random selection of 50 patients ( 2156 epochs). Of the 24447 epochs available, 6157 (25\%) were excluded by the artifact rejection algorithm. Exclusion most often resulted from muscle artifacts (13\%), followed by high-amplitude artifacts (9.9\%) and flat channels (2.6\%). Illustrative examples for each type of artifact are shown in Figure 3.1. An additional 69 out of 2549 epochs with BSAR $\geq 4$ were excluded after a visual check on high-amplitude artifacts.

\subsubsection{Continuity and burst suppression amplitude ratio calculation}

Examples for the calculation of $\mathrm{BCl}$ and $\mathrm{BSAR}$ are shown in Figure 3.2. Note that the BSAR clearly distinguishes patterns with gradual transitions between bursts and suppressions (panels A, C) from burst-suppression with epileptiform bursts (panel B) and burst-suppression with identical bursts (panel D). The highest BSAR value found in patients with a good outcome was 6.11.

\subsubsection{Evolution of qEEG features}

As known from visual analyses, shortly after cardiac arrest, almost all patients have discontinuous traces ${ }^{1}$. In $96 \%$ patients that eventually had a good outcome, the EEG improved towards a (nearly) continuous pattern $(\mathrm{BCl} \geq 0.9)$ within 72 hours, whereas this occurred in only $65 \%$ of cases with poor outcome. If reached, time to continuity $(\mathrm{BCl}$ $\geq 0.9$ ) was longer in the poor outcome group ( $34 \pm 14$ hrs. vs. $23 \pm 13$ hrs., $p<0.001$ ). 
Table 3.2: Clinical characteristics of patients included.

\begin{tabular}{lccc}
\hline Characteristic & $\begin{array}{c}\text { Good outcome } \\
\text { (CPC 1-2) }\end{array}$ & $\begin{array}{c}\text { Poor outcome } \\
\text { (CPC 3-5) }\end{array}$ & P-value \\
\hline Number of patients & $259(46 \%)$ & $300(54 \%)$ & \\
Age & $60(29-88)$ & $68(19-93)$ & $<0.001$ \\
Female & $60(23 \%)$ & $80(27 \%)$ & 0.34 \\
Out of hospital cardiac arrest & $238(92 \%)$ & $263(88 \%)$ & 0.10 \\
Primary cardiac cause of arrest & $230(94 \%)$ & $203(76 \%)$ & $<0.001$ \\
VF as initial rhythm & $233(92 \%)$ & $160(58 \%)$ & $<0.001$ \\
Treated with hypothermia $\left(33^{\circ} \mathrm{C}\right)$ & $165(64 \%)$ & $207(69 \%)$ & 0.17 \\
Treated with propofol & $240(95 \%)$ & $260(89 \%)$ & 0.01 \\
$\quad$ Max. propofol rate $(\mathrm{mg} / \mathrm{kg} / \mathrm{h})$ & $2.95(0.18-7.14)$ & $2.67(0.53-6.15)$ & 0.03 \\
Treated with midazolam & $85(34 \%)$ & $106(36 \%)$ & 0.51 \\
$\quad$ Max. midazolam rate $(\mu \mathrm{gg} / \mathrm{kg} / \mathrm{h})$ & $92(27-356)$ & $105(22-556)$ & 0.70 \\
Treated with fentanyl & $117(46 \%)$ & $154(53 \%)$ & 0.13 \\
$\quad$ Max. fentanyl rate $(\mu \mathrm{g} / \mathrm{kg} / \mathrm{h})$ & $1.72(0.67-5.18)$ & $1.38(0.56-4.71)$ & 0.002 \\
Treated with remifentanil & $17(7 \%)$ & $28(10 \%)$ & 0.23 \\
$\quad$ Max. remifentanil rate $(\mu \mathrm{gg} / \mathrm{kg} / \mathrm{h})$ & $6.67(2.5-15)$ & $3.2(1.2-14)$ & 0.01 \\
Treated with morphine & $110(44 \%)$ & $103(35 \%)$ & 0.05 \\
$\quad$ Max. morphine rate $(\mu \mathrm{g} / \mathrm{kg} / \mathrm{h})$ & $25(9-113)$ & $26(12-65)$ & 0.02 \\
Median nerve SSEP & $39(15 \%)$ & $198(66 \%)$ & $<0.001$ \\
Bilaterally absent N20 response & $0(0 \%)$ & $91(30 \%)$ & $<0.001$ \\
EEG start time (hours) & $9(2-28)$ & $9(1-63)$ & 0.90 \\
EEG end time (hours) & $64(7-260)$ & $65(3-223)$ & 0.51 \\
\hline
\end{tabular}

Numbers are displayed as median (range), unless otherwise indicated. EEG start and end times are relative to the time of cardiac arrest. SSEP: somatosensory evoked potential, VF: ventricular fibrillation. 
A

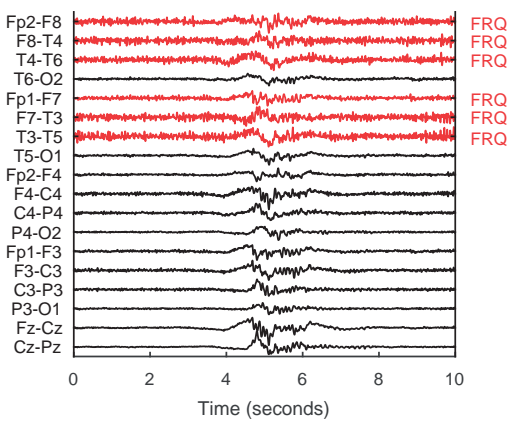

C

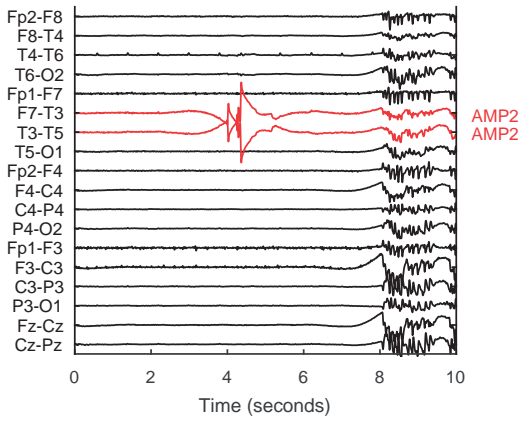

B

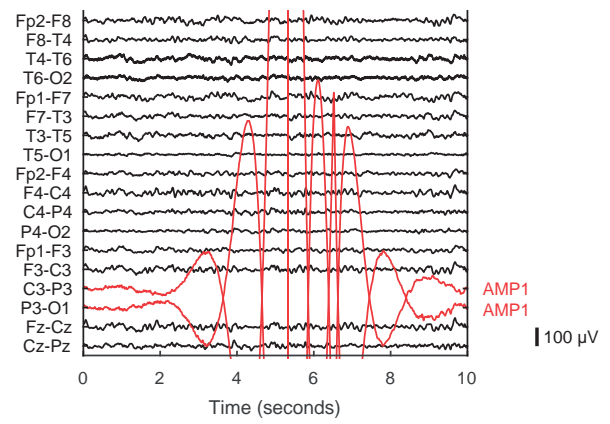

D

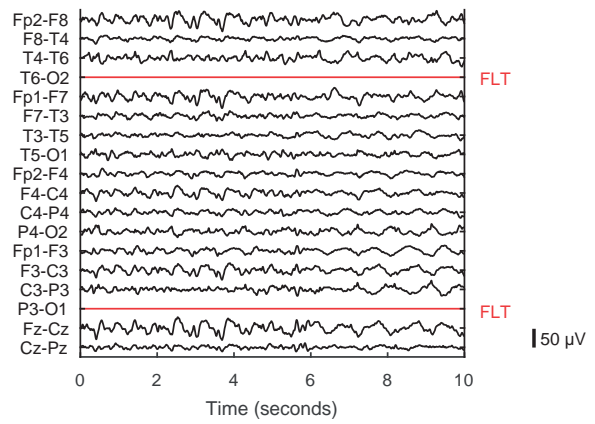

Figure 3.1: Examples of artifact rejection algorithm. Ten-second samples taken from five-minute epochs are shown. Excluded channels are displayed as red lines, where the type of artifact is indicated by the text on the right. A: Muscle artifacts (FRQ) in 6 channels. B: Two channels with high-amplitude artifacts above the absolute threshold of $500 \mu \mathrm{V}$ (AMP1). C: Two channels with high-amplitude artifacts above the relative threshold (AMP2). D: Two flat channels (FLT). Since all of these examples have at least 12 artifact-free channels remaining, none of these epochs were excluded completely from further analysis. 

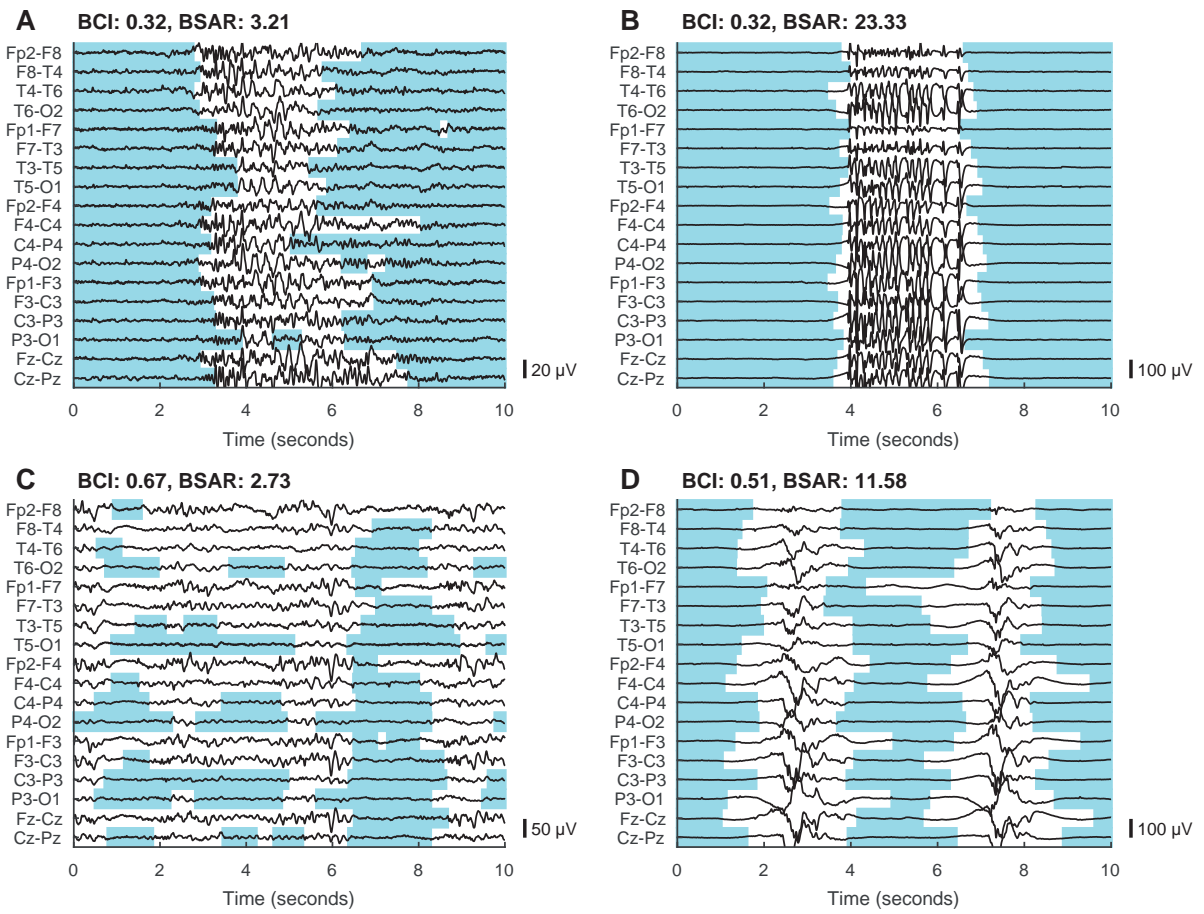

Figure 3.2: Four examples of EEG patterns with corresponding $\mathrm{BCl}$ and $\mathrm{BSAR}$ values. Ten-second samples taken from five-minute epochs are shown. The indicated qEEG features were extracted from the ten-second sample only. Segments classified as ‘suppression' are indicated by blue highlights. A: Burst with physiological bursts content, where the burst amplitude is low relative to the suppressions. This is reflected by the low BSAR value (3.21). B: Burst with epileptiform morphology on a suppressed background. Note that $\mathrm{BCl}$ is the same as for example A, but with significantly higher BSAR (23.33). C: Pattern with gradual transitions between suppressions and bursts and relatively low burst amplitude. In this case, BSAR is low (2.73). D: Burst-suppression with identical bursts, again with a high BSAR (11.58). 
Of all patients with a poor outcome, 97 (32\%) had a BSAR $\geq 6.12$ during their EEG recording. In $85 \%$ of these patients, a value above the threshold was observed in the first 24 hours after cardiac arrest, and $66 \%$ reached their maximum BSAR within 24 hours. Figure 3.3 shows the evolution of $\mathrm{BCl}$ and $\mathrm{BSAR}$ in the first 72 hours after cardiac arrest.

\subsubsection{Single features predicting good or poor outcome}

The single feature that best predicted good outcome was the background continuity index. At a specificity of $90 \%, \mathrm{BCl} \geq 0.92$ at 24 hours predicted good outcome with a sensitivity of $53 \%$, which differed not statistically significant from any other time point between 3 and 30 hours after cardiac arrest. Relevant discrimination for good outcome prediction using $\mathrm{BCl}$ was only achieved within 47 hours. Thereafter, specificity was never higher than $90 \%$.

The single feature that best predicted a poor outcome, without false positives, was the BSAR. Its sensitivity was $38 \%$ at 24 hours, which was not significantly different from any other time point between 2 and 34 hours, and decreased for later time points. Irrespective of timing with respect to cardiac arrest, taking all EEG epochs for all patients into account, a BSAR $\geq 6.12$ always predicted a poor outcome. At 11 hours or later, a $\mathrm{BCl}$ $<0.014$ always predicted a poor outcome.

Test characteristics for the individual predictors and their combinations at $12,24,48$, and 72 hours are summarized in Table 3.3.

\subsubsection{Combinations of features for prediction of outcome}

Combination of features allowed detection of more patients with good or poor outcome than single features, at equal specificity. Sensitivity for the prediction of good outcome at 24 hours could be improved from $53 \%$ to $57 \%$ by using the prediction model instead of the $\mathrm{BCl}$.

The prediction of poor outcome using either $\mathrm{BCl}<0.014$ or $\mathrm{BSAR} \geq 6.11$ was without false positives for each time point between 11 and 72 hours after cardiac arrest. Their combined sensitivity at 12 hours after cardiac arrest was $46 \%$. By using the prediction model instead of the individual features, sensitivity at 12 hours improved to $50 \%$, at $100 \%$ specificity.

Just like the individual features, the model predictions suggests that the EEG has its highest prognostic value in in the first two days after cardiac arrest. The AUC of the ROC curves of the model predictions differed with no statistical significance between 1 and 42 hours after cardiac arrest, and decreased thereafter. The AUC of the ROC curve was $0.86(0.82-0.90)$ at 12 hours, and $0.87(0.83-0.91)$ at 24 hours, as shown in Figure 3.4.

\subsubsection{Influence of sedative medication}

The association of propofol and midazolam doses with the quantitative EEG features was the opposite of what could be expected based on their pharmacological characteristics (Figure 3.5). For both propofol and midazolam doses, there was a positive correlation with the background continuity index and no statistically significant correlation with the burst-suppression amplitude ratio. Also for fentanyl, remifentanil, and morphine, increasing doses were associated with more favorable values of $\mathrm{BCl}$ and $\mathrm{BSAR}$, if 
A $\quad \mathrm{BCl}$, all patients with good outcome (CPC 1-2)

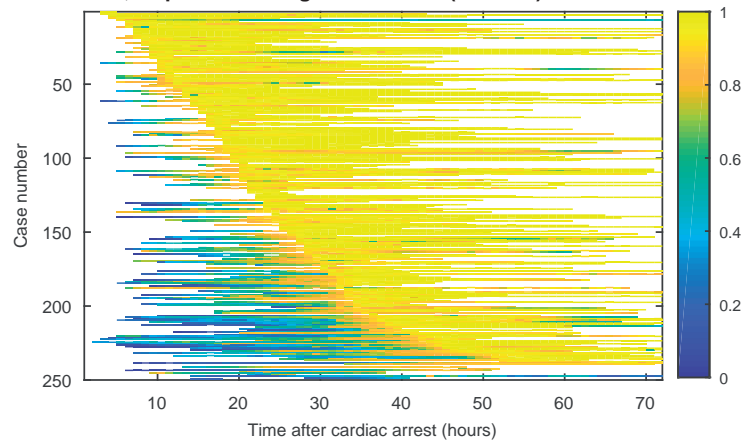

B $\quad \mathrm{BCl}$, all patients with poor outcome (CPC 3-5)

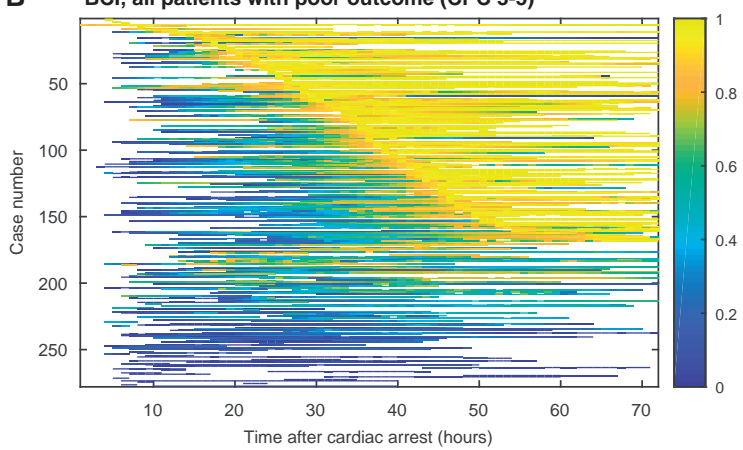

C BSAR, all patients with value $\geq 6.12$

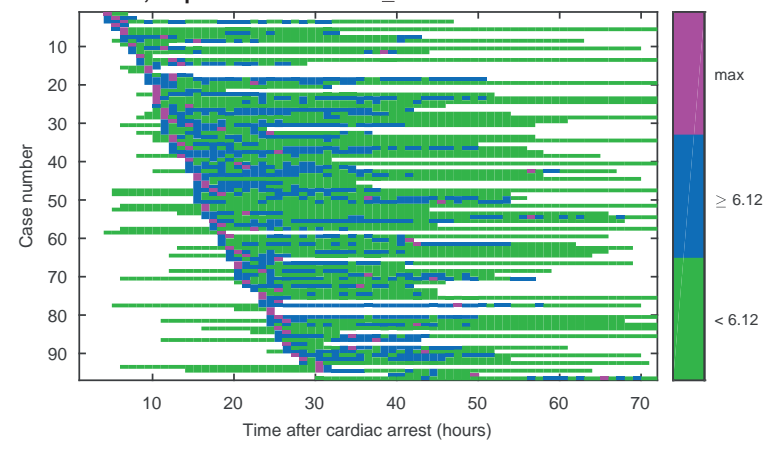

Figure 3.3: Evolution of qEEG features in the first 72 hours after cardiac arrest. A: Evolution of $\mathrm{BCl}$ for all cases with a good outcome (CPC 1-2). Each horizontal line represents one case. Dark blue color indicates a suppressed EEG $(\mathrm{BCl}=0)$, and bright yellow indicates continuous, normal amplitude background $(\mathrm{BCl}=1)$, as follows from the color bar on the right. For values missing due to excluded epochs, linear interpolation was applied. Cases were primarily sorted by the time at which the EEG background was nearly continuous for the first time $(\mathrm{BCl} \geq 0.9)$, and secondarily by their maximally achieved $\mathrm{BCl}$ value. $\mathrm{B}$ : Evolution of $\mathrm{BCl}$ for all cases with a poor outcome (CPC 3-5). C: evolution of BSAR for all patients with a value above the threshold for poor outcome (6.11). Green color indicates values below the threshold, blue color values above the threshold, and purple color the maximum value per patient. Cases were primarily sorted by the timing of the first BSAR value above the threshold, and secondarily by the maximally achieved BSAR value. 


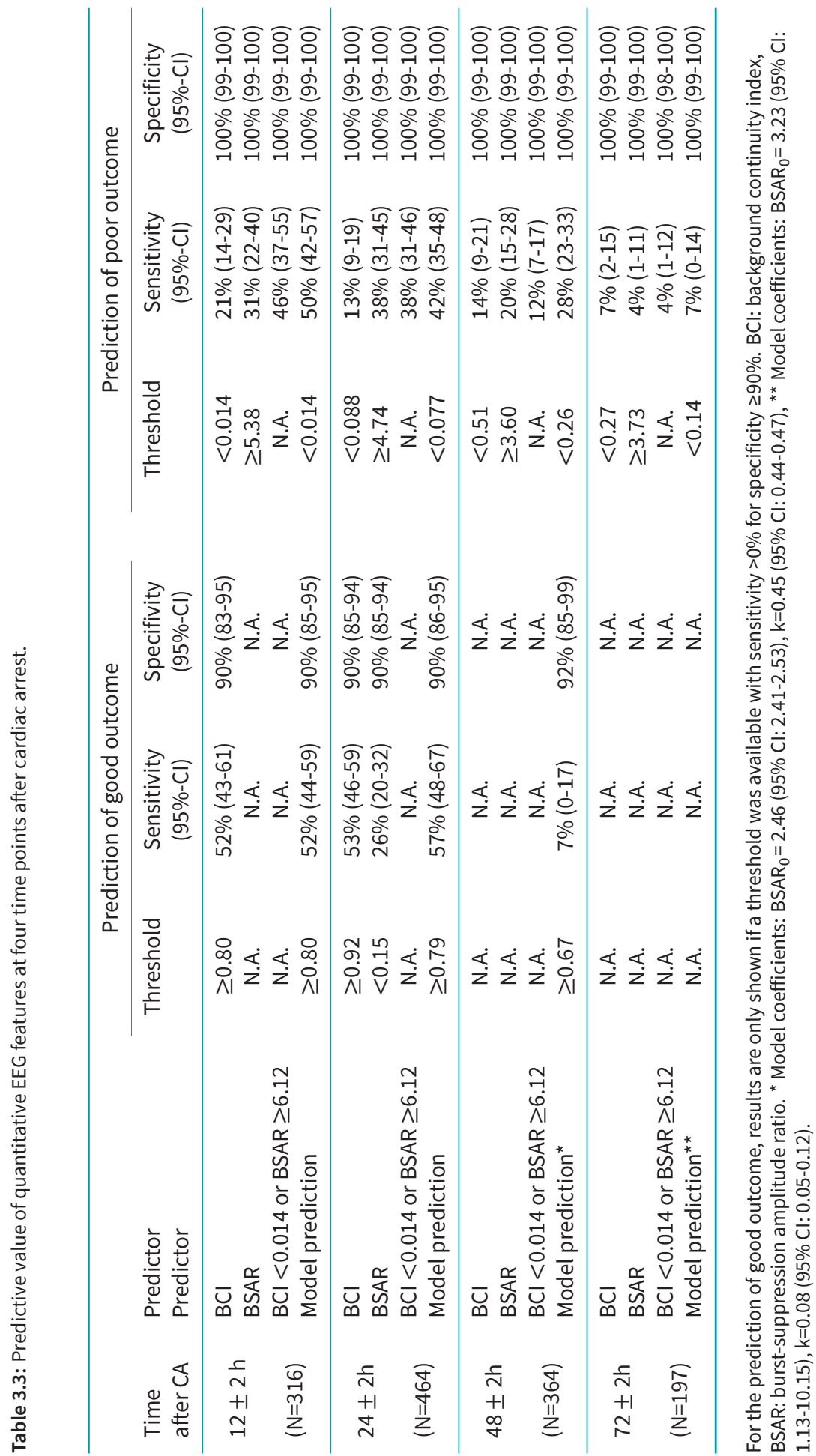


A Time: $12 \pm 2$ hours after cardiac arrest

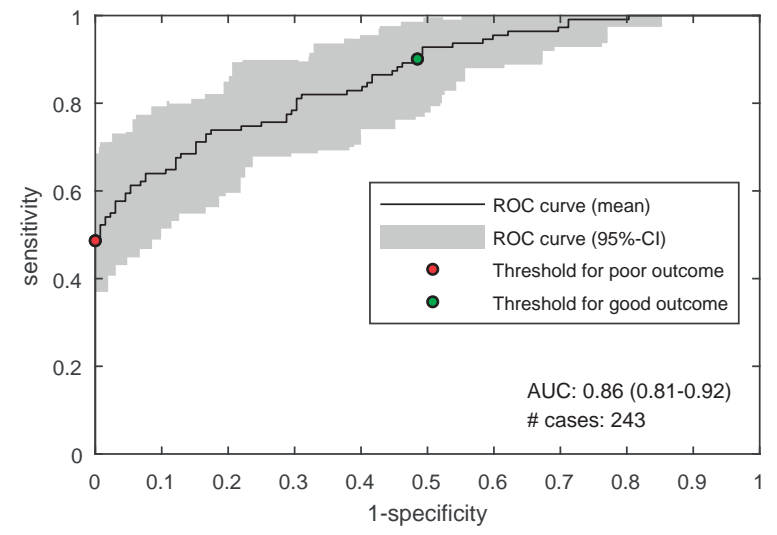

B Time: $24 \pm 2$ hours after cardiac arrest

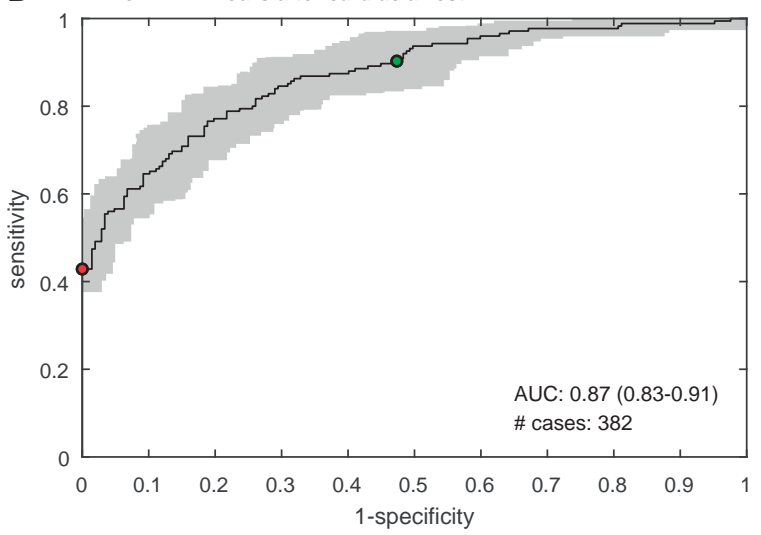

Figure 3.4: ROC-curves for the prediction model including both $\mathrm{BCl}$ and BSAR. Green dots indicate the threshold value for prediction of good outcome, with maximized sensitivity at $90 \%$ specificity. Red dots indicate the threshold values for prediction of poor outcome, with maximized sensitivity for $100 \%$ specificity. The curves shown were constructed by threshold averaging of the ROC curves resulting from each fold of the cross-validation procedure. Confidence bands were calculated using bootstrapping estimates ( $N=2000)$. A: ROC-curve for $12 \pm 2$ hours after cardiac arrest. Model coefficients are $\mathrm{BSAR}_{0}=5.43$ (95\% Cl: 5.39-5.53), $\mathrm{k}=264$ (95\% Cl: 166-377). B: ROC-curve for $24 \pm 2$ hours after cardiac arrest. Model coefficients are BSAR $=4.49(95 \%$ Cl: 4.45-4.55), $\mathrm{k}=1.32$ (95\% Cl: 1.23-1.40).). AUC: area under curve. 

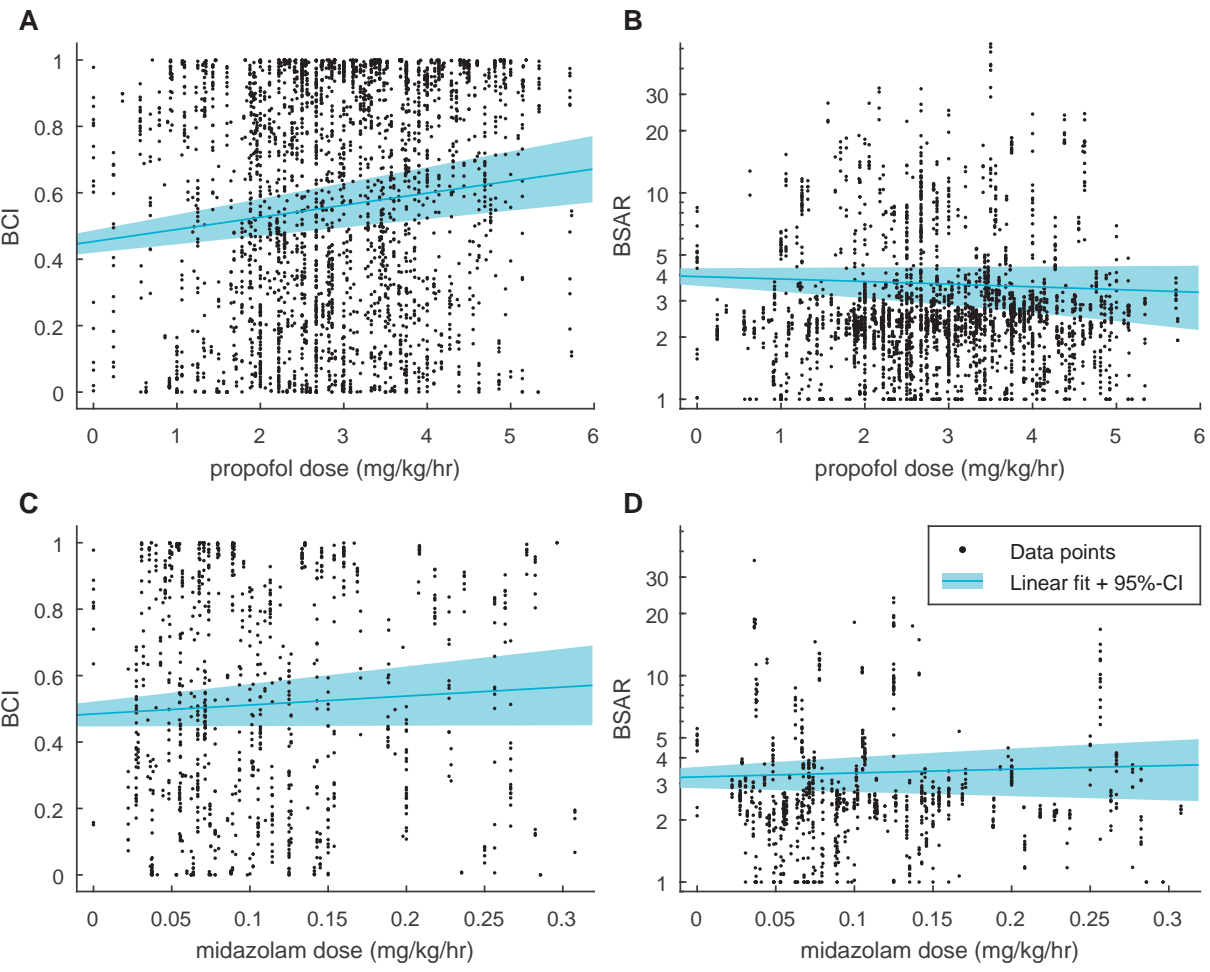

Figure 3.5: Association between doses of sedative medication and qEEG features in the first 24 hours. Quantitative EEG features plotted as function of the dose of sedative drugs in $\mathrm{mg} / \mathrm{kg} / \mathrm{h}$. Each subplot includes a linear fit with $95 \%$ confidence intervals. Note the apparent vertical lines in each of the subplots, caused by multiple values extracted in the same patient for constant level of sedation. A: $\mathrm{BCl}$ versus propofol dose $\left(\beta=3.6 \pm 1.1 \cdot 10^{-2}, R^{2}=1.3 \cdot 10^{-2}\right)$. B: BSAR versus propofol dose $\left(\beta=-1.1 \pm 1.3 \cdot 10^{-1}, R^{2}=8.8 \cdot 10^{-4}\right)$. $\mathrm{C}$ : $\mathrm{BCl}$ versus midazolam dose $\left(\beta=2.7 \pm 2.6 \cdot 10^{-1}, R^{2}=4.1 \cdot 10^{-3}\right)$. D: BSAR versus midazolam dose $\left(\beta=1.46 \pm 2.66, R^{2}=1.1 \cdot 10^{-3}\right)$.

statistically significant (Supplementary Figure 3.A.1). The proportion of variance in the quantitative EEG parameters explained by the dose of sedative medication was very low $\left(R^{2} \leq 0.15\right.$ in any case).

\subsection{Discussion}

We show that the background continuity index and the burst-suppression amplitude ratio are straightforward, quantitative EEG-measures that assist in the prediction of outcome after cardiac arrest. Our results confirm that return of EEG background continuity within 24 hours indicates a good outcome. Otherwise, a lack of background continuity predicts poor outcome without false positives, except for the first 10 hours after cardiac arrest. An amplitude ratio between 'bursts' and suppressions of 6.12 and above is invariably associated with a poor outcome, irrespective of timing with respect to cardiac arrest. The accuracy of outcome prediction increases significantly by using combinations of both features. We found no evidence that the effects of sedative med- 
ication led to falsely pessimistic outcome predictions.

\subsubsection{Comparison with previous work}

We quantify and generalize key findings of studies that used visual assessment of the EEG for the prediction of outcome after cardiac arrest ${ }^{2-4}$. A high BSAR value captures burst-suppression with identical bursts ${ }^{5}$, GPDs on a suppressed background ${ }^{6}$, and "highly malignant" EEG patterns of the continuum in between ${ }^{24,25}$. For the remainder of EEG patterns, the $\mathrm{BCl}$ allows for reliable outcome predictions for various degrees of background continuity, whereas visual assessment seem limited to cases with either absent $(\mathrm{BCl}=0)$ or continuous $(\mathrm{BCl}=1)$ background activity ${ }^{1}$.

Although various outcome predictors of postanoxic coma have been proposed, only EEG-related measures have been shown to be valuable in prediction of good outcome ${ }^{1,}$ ${ }^{24}$. For the prediction of good outcome at 12 hours, the background continuity index as single feature performs at least as good as visual EEG analysis in terms of sensitivity and specificity ${ }^{3}$.

Our quantitative EEG features are suitable for the prediction of poor outcome in clinical practice, given their zero false positive rates. Their sensitivities, reaching $45-57 \%$ in combination, are good as compared to other highly-specific predictors, such as bilaterally absent N20-responses (25-57\%), absent corneal reflexes (22-44\%), absent pupillary light reflexes (19-36\% ${ }^{26}$, and an unfavorable EEG pattern identified by visual assessment $(22-26 \%)^{3}$. By using only two intuitively clear qEEG features, the accuracy of our prediction model is essentially the same as the Cerebral Recovery Index, the most accurate quantitative EEG index for the prediction of outcome after cardiac arrest reported so far ${ }^{11}$.

\subsubsection{Background continuity and recovery from anoxic brain injury}

In neonates with hypoxic-ischemic encephalopathy, the prognostic value of EEG background continuity has been well established ${ }^{27,28}$. Although some studies have stressed the importance of restoration of EEG background continuity for recovery from postanoxic encephalopathy in adults ${ }^{14,29,30}$, this is the first systematic analysis in a large prospectively collected cohort. Since EEG activity is mainly a reflection of cortical synaptic activity ${ }^{31}$, which is very sensitive to effects of ischemia ${ }^{32}$, interruption of EEG activity immediately after cardiac arrest results from massive cortical synaptic arrest. The return of EEG background continuity reflects a gradual recovery of synaptic activity ${ }^{33}$. Our results suggest that restoration of synaptic functioning within 24 hours is a necessary condition for regaining consciousness and a good neurologic outcome. This is in agreement with studies in in vitro models, showing that absence of synaptic recovery within 24 hours after an hypoxic insult is strongly associated with irreversible neuronal damage ${ }^{34}$.

\subsubsection{Role of the burst-suppression amplitude ratio}

The amplitude ratio between bursts and suppressions was a reliable predictor for poor outcome at each of the investigated time points, with highest sensitivity at 24 hours after cardiac arrest. Certain burst-suppression patterns in postanoxic encephalopathy have been recognized as invariably associated with poor outcome ${ }^{2,5}$. Not only does 
the amplitude ratio identify malignant burst patterns, it also captures generalized periodic discharges on a suppressed background, which are equally associated with poor outcome $^{6}$. Since suppressions are easy to detect quantitatively, the amplitude ratio between 'bursts' and suppressions is a straightforward measure, avoiding well known challenges in detection of bursts ${ }^{35}$ and epileptiform discharges ${ }^{36,37}$. Our results indicate that a BSAR $\geq 6.12$ always leads to a poor outcome, irrespective of the time relative to the anoxic event.

Our study indicates that patients with severe postanoxic brain injury may have large amplitude fluctuations in their EEG-patterns, but this does not explain the underlying mechanisms. A candidate mechanism for these patterns is a change in the synaptic excitation-inhibition ratio. A transient period of anoxia can induce long-term potentiation of excitatory neurotransmission ${ }^{38}$. We recently incorporated this effect in a computational model, and showed that this resulted in generalized periodic discharges or burst-suppression patterns with high amplitudes on a suppressed background ${ }^{33}$. The increased excitation-inhibition ratio may subsequently contribute to excitotoxicity, massive secondary cell death, and poor outcome ${ }^{38}$.

\subsubsection{Influence of sedative medication}

Based on their pharmacological effects, we expected propofol and midazolam to decrease continuity and to increase the burst-suppression amplitude ratio, which both could lead to false prediction of poor outcome. We observed otherwise: increasing doses of sedative medication were associated with more favorable values of $\mathrm{BCl}$ and BSAR. Most likely, the severity of postanoxic brain injury is a confounding variable in this respect. Patients without severe brain injury are more likely to have a continuous EEG, with BSAR close to one, but are also more likely to have arousals and higher sedative requirements. Besides, the variance in the quantitative EEG features explained by the doses of sedatives was very low. These findings indicate that the influence of the severity of postanoxic injury on the EEG is much stronger than those for sedative drugs, in the typical doses administered.

\subsubsection{Limitations}

An important limitation of prognostication studies in comatose patients after cardiac arrest is the risk of a self-fulfilling prophecy ${ }^{39}$. This is the bias occurring when the treating physicians are not blinded to the results of the outcome predictor and use it to make a decision to withdraw life-sustaining treatment. To minimize this risk, decisions on treatment withdrawal were based on international guidelines including bilaterally absent SSEP, absent or extensor motor responses, and absent brain stem reflexes ${ }^{19,20}$, and the EEG within the first 72 hours after cardiac arrest was not taken into account. Furthermore, the analysis of quantitative EEG features in this study was performed offline, and treating physicians did not have these parameters available when making decisions on withdrawal of treatment.

To some degree, our definition of 'suppressions' is arbitrary. Standardized critical care EEG terminology defines the amplitude cutoff value of $10 \mu \mathrm{V}^{7}$, but does not provide a minimum duration for suppressions. The choice for a minimum suppression duration is a trade-off between false detection of segments with spontaneous reductions in am- 
plitude and not detecting brief suppressions in between GPDs. Doubling the minimum suppression duration would decrease sensitivity for the prediction of poor outcome, since GPDs on a suppressed background with frequency $1-2 \mathrm{~Hz}$ would then be classified as 'continuous'. A reduction of the minimum suppression duration would increase the fraction of patterns classified by quantitative analysis as 'discontinuous' $(\mathrm{BCl}<0.9)$ that would have been classified as 'continuous' by visual analysis. It should be noted that lowering the minimum suppression duration to $250 \mathrm{~ms}$, as tested during development of the algorithm, did not significantly influence the accuracy for the prediction of poor outcome.

The presented qEEG features critically depend on the rejection of artifacts. Despite the use of an artifact rejection algorithm, we cannot exclude the possibility that our results have been influenced by remaining artifacts. The most important risk is a false prediction of poor outcome. The risk of an underestimation of $\mathrm{BCl}$ seems negligible, since the algorithm rejects flat channels efficiently. Overestimation of the BSAR, however, is still possible, since the artifact rejection algorithm does not exclude all high-amplitude artifacts. For this reason, all epochs with BSAR $\geq 4$ were checked visually, after which another $2.5 \%$ were excluded from further analysis. Therefore, we stress that our qEEG measures should be used as a tool to assist in visual interpretation of the EEG, allowing for assessment of any remaining artifacts.

\section{References}

1. Hofmeijer J, Van Putten MJAM. EEG in postanoxic coma: Prognostic and diagnostic value. Clinical Neurophysiology 2016; 127: 2047-2055.

2. Sivaraju A, Gilmore EJ, Wira CR, Stevens A, Rampal N, Moeller JJ, et al. Prognostication of post-cardiac arrest coma: early clinical and electroencephalographic predictors of outcome. Intensive Care Medicine 2015; 41: 1264-1272.

3. Sondag L, Ruijter BJ, Tjepkema-Cloostermans MC, Beishuizen A, Bosch FH, van Til JA, et al. Early EEG for outcome prediction of postanoxic coma: prospective cohort study with costminimization analysis. Critical Care 2017; 21: 111.

4. Spalletti M, Carrai R, Scarpino M, Cossu C, Ammannati A, Ciapetti M, et al. Single electroencephalographic patterns as specific and time-dependent indicators of good and poor outcome after cardiac arrest. Clinical Neurophysiology 2016; 127: 2610-2617.

5. Hofmeijer J, Tjepkema-Cloostermans MC, van Putten MJAM. Burst-suppression with identical bursts: A distinct EEG pattern with poor outcome in postanoxic coma. Clinical Neurophysiology 2014; 125: 947-954.

6. Ruijter BJ, van Putten MJAM, Hofmeijer J. Generalized epileptiform discharges in postanoxic encephalopathy: Quantitative characterization in relation to outcome. Epilepsia 2015; 56: 1845-1854.

7. Hirsch LJ, LaRoche SM, Gaspard N, Gerard E, Svoronos A, Herman ST, et al. American Clinical Neurophysiology Society's Standardized Critical Care EEG Terminology. Journal of Clinical Neurophysiology 2013; 30: 1-27.

8. Gaspard N, Hirsch LJ, LaRoche SM, Hahn CD, Westover MB, C C E M R Consortium. Interrater agreement for Critical Care EEG Terminology. Epilepsia 2014; 55: 1366-1373.

9. Westhall E, Rosén I, Rossetti AO, van Rootselaar AF, Wesenberg Kjaer T, Friberg H, et al. Interrater variability of EEG interpretation in comatose cardiac arrest patients. Clinical Neurophysiology 2015; 126: 2397-2404. 
10. Swisher CB, Sinha SR. Utilization of Quantitative EEG Trends for Critical Care Continuous EEG Monitoring. Journal of Clinical Neurophysiology 2016; 33: 538-544.

11. Tjepkema-Cloostermans MC, Hofmeijer J, Beishuizen A, Hom HW, Blans MJ, Bosch FH, et al. Cerebral Recovery Index: Reliable help for prediction of neurologic outcome after cardiac arrest. Critical Care Medicine 2017; 45: 789-797.

12. Zubler F, Bandarabadi M, Kurmann R, Steimer A, Gast H, Schindler KA. Quantitative EEG in the Intensive Care Unit. Epileptologie 2016; 33: 166-172.

13. Noirhomme Q, Lehembre R, del Rosario Lugo Z, Lesenfants D, Luxen A, Laureys S, et al. Automated analysis of background EEG and reactivity during therapeutic hypothermia in comatose patients after cardiac arrest. Clinical EEG and Neuroscience 2014; 45: 6-13.

14. Wennervirta JE, Ermes MJ, Tiainen SM, Salmi TK, Hynninen MS, Särkelä MOK, et al. Hypothermia-treated cardiac arrest patients with good neurological outcome differ early in quantitative variables of EEG suppression and epileptiform activity. Critical Care Medicine 2009; 37: 2427-2435.

15. Cloostermans MC, van Meulen FB, Eertman CJ, Hom HW, van Putten MJAM. Continuous electroencephalography monitoring for early prediction of neurological outcome in postanoxic patients after cardiac arrest: A prospective cohort study. Critical Care Medicine 2012; 40: 2867-2875.

16. Hofmeijer J, Beernink TMJ, Bosch FH, Beishuizen A, Tjepkema-Cloostermans MC, Van Putten MJAM. Early EEG contributes to multimodal outcome prediction of postanoxic coma. Neurology 2015; 85: 137-143.

17. Tjepkema-Cloostermans MC, Hofmeijer J, Trof RJ, Blans MJ, Beishuizen A, van Putten MJAM. Electroencephalogram predicts outcome in patients with postanoxic coma during mild therapeutic hypothermia. Critical Care Medicine 2015; 43: 159-167.

18. Tjepkema-Cloostermans MC, van Meulen FB, Meinsma G, Van Putten MJAM. A Cerebral Recovery Index (CRI) for early prognosis in patients after cardiac arrest. Critical Care 2013; 17: R252.

19. Sandroni C, Cariou A, Cavallaro F, Cronberg T, Friberg H, Hoedemaekers C, et al. Prognostication in comatose survivors of cardiac arrest: An advisory statement from the European Resuscitation Council and the European Society of Intensive Care Medicine. Resuscitation 2014; 85: 1779-1789.

20. Wijdicks EFM, Hijdra A, Young GB, Bassetti CL, Wiebe S. Practice parameter: prediction of outcome in comatose survivors after cardiopulmonary resuscitation (an evidence-based review): report of the Quality Standards Subcommittee of the American Academy of Neurology. Neurology 2006; 67: 203-210.

21. Jennett $B$, Bond $M$. Assessment of outcome after severe brain damage: A practical scale. The Lancet 1975; 305: 480-484.

22. Veselis RA, Reinsel R, Marino P, Sommer S, Carlon GC. The effects of midazolam on the EEG during sedation of critically ill patients. Anaesthesia 1993; 48: 463-470.

23. ter Horst $\mathrm{H}$, Brouwer O, Bos A. Burst suppression on amplitude-integrated electroencephalogram may be induced by midazolam: a report on three cases. Acta Paediatrica 2004; 93 : 559-563.

24. Rossetti AO, Tovar Quiroga DF, Juan E, Novy J, White RD, Ben-Hamouda N, et al. Electroencephalography Predicts Poor and Good Outcomes After Cardiac Arrest. Critical Care Medicine 2017; 45: e674-e682.

25. Westhall E, Rossetti AO, van Rootselaar AF, Wesenberg Kjaer T, Horn J, Ullén S, et al. Standardized EEG interpretation accurately predicts prognosis after cardiac arrest. Neurology 2016; 86: 1482-1490.

26. Golan E, Barrett K, Alali AS, Duggal A, Jichici D, Pinto R, et al. Predicting Neurologic Outcome After Targeted Temperature Management for Cardiac Arrest. Critical Care Medicine 2014; 42: 
1919-1930.

27. Menache CC, Bourgeois BFD, Volpe JJ. Prognostic value of neonatal discontinuous EEG. Pediatric Neurology 2002; 27: 93-101.

28. Walsh BH, Murray DM, Boylan GB. The use of conventional EEG for the assessment of hypoxic ischaemic encephalopathy in the newborn: A review. Clinical Neurophysiology 2011; 122: 1284-1294.

29. Jørgensen E, Holm S. The natural course of neurological recovery following cardiopulmonary resuscitation. Resuscitation 1998; 36: 111-122.

30. Oh SH, Park KN, Shon YM, Kim YM, Kim HJ, Youn CS, et al. Continuous amplitude-integrated electroencephalographic monitoring is a useful prognostic tool for hypothermia-treated cardiac arrest patients. Circulation 2015; 132: 1094-1103.

31. Buzsáki G, Anastassiou CA, Koch C. The origin of extracellular fields and currents - EEG, ECoG, LFP and spikes. Nature Reviews Neuroscience 2012; 13: 407-420.

32. Hofmeijer J, van Putten MJAM. Ischemic cerebral damage: an appraisal of synaptic failure. Stroke 2012; 43: 607-615.

33. Ruijter BJ, Hofmeijer J, Meijer HGE, van Putten MJAM. Synaptic damage underlies EEG abnormalities in postanoxic encephalopathy: A computational study. Clinical Neurophysiology 2017; 128: 1682-1695.

34. Le Feber J, Erkamp N, van Putten MJAM, Hofmeijer J. Loss and recovery of functional connectivity in cultured cortical networks exposed to hypoxia. Journal of Neurophysiology 2017; 118: 394-403.

35. Westover MB, Shafi MM, Ching S, Chemali JJ, Purdon PL, Cash SS, et al. Real-time segmentation of burst suppression patterns in critical care EEG monitoring. Journal of Neuroscience Methods 2013; 219: 131-141.

36. Haider HA, Esteller R, Hahn CD, Westover MB, Halford JJ, Lee JW, et al. Sensitivity of quantitative EEG for seizure identification in the intensive care unit. Neurology 2016; 87: 935-944.

37. Halford JJ. Computerized epileptiform transient detection in the scalp electroencephalogram: Obstacles to progress and the example of computerized ECG interpretation. Clinical Neurophysiology 2009; 120: 1909-1915.

38. Szatkowski M, Attwell D. Triggering and execution of neuronal death in brain ischaemia: Two phases of glutamate release by different mechanisms. Trends in Neurosciences 1994; 17: 359-365.

39. Geocadin RG, Peberdy MA, Lazar RM. Poor survival after cardiac arrest resuscitation. Critical Care Medicine 2012; 40: 979-980. 


\section{A Supplementary figure}

A

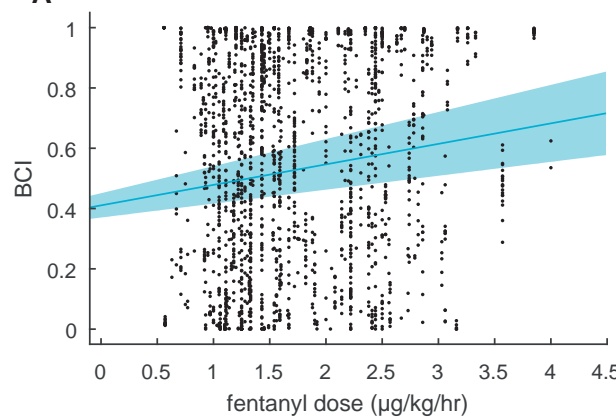

C

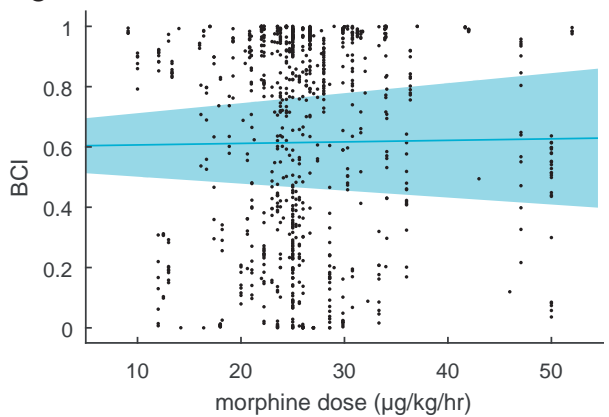

E

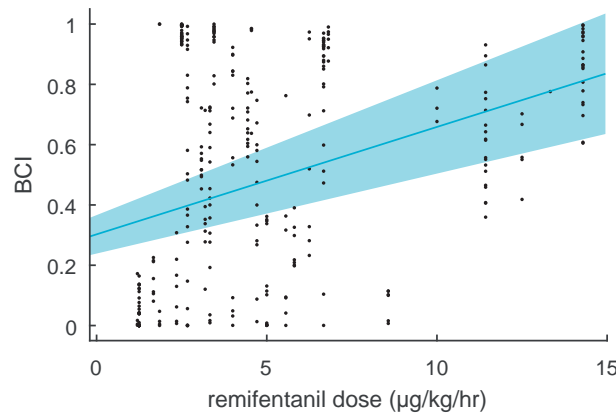

B

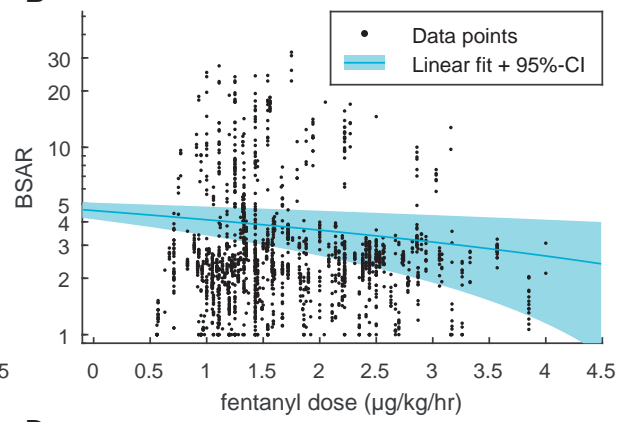

D

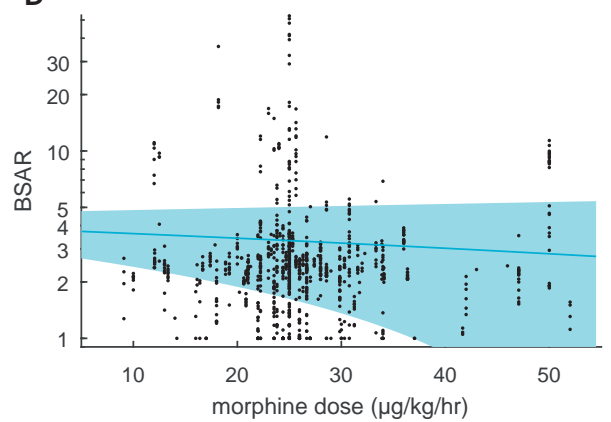

$\mathbf{F}$

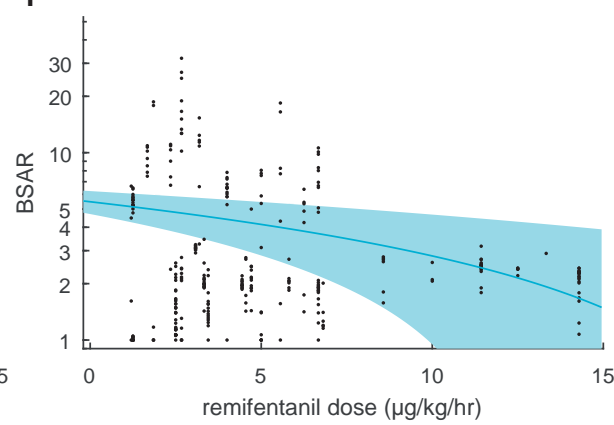

Figure 3.A.1: Association between doses of sedative medication and qEEG features in the first 24 hours (part 2). Quantitative EEG features plotted as function of the dose of sedative drugs in $\mu \mathrm{g} / \mathrm{kg} / \mathrm{h}$. Each subplot includes a linear fit with $95 \%$ confidence intervals. Note the apparent vertical lines in each of the subplots, caused by multiple values extracted in the same patient for constant level of sedation. A: $\mathrm{BCl}$ versus fentanyl dose $\left(\beta=6.8 \pm 2.2 \cdot 10^{-2}, R^{2}=1.9 \cdot 10^{-2}\right)$. B: BSAR versus fentanyl dose $\left(\beta=-4.9 \pm 2.5 \cdot 10^{-1}, R^{2}=\right.$ $\left.7.4 \cdot 10^{-3}\right)$. C: $\mathrm{BCl}$ versus morphine dose $\left(\beta=0.5 \pm 2.8 \cdot 10^{-3}, R^{2}=1.3 \cdot 10^{-4}\right)$. D: BSAR versus morphine dose $\left(\beta=-2.0 \pm 3.2 \cdot 10^{-2}, R^{2}=1.6 \cdot 10^{-3}\right)$. E: $\mathrm{BCl}$ versus remifentanil dose $\left(\beta=3.6 \pm 0.9 \cdot 10^{-2}, R^{2}=\right.$ $\left.1.5 \cdot 10^{-1}\right)$. F: BSAR versus remifentanil dose $\left(\beta=-2.7 \pm 0.9 \cdot 10^{-1}, R^{2}=6.5 \cdot 10^{-2}\right)$. 



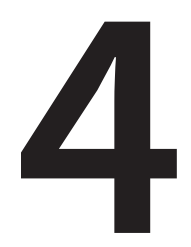

Generalized epileptiform
discharges in postanoxic encephalopathy: quantitative characterization in relation to outcome 


\section{Abstract}

Objective

Methods

Results

Significance

Electrographic status epilepticus is observed in 10 to $35 \%$ of patients with postanoxic encephalopathy. It remains unclear which electrographic seizure patterns indicate possible recovery, and which are a mere reflection of severe ischemic encephalopathy, where treatment would be futile. We aimed to identify quantitative EEG features with prognostic significance.

From continuous EEG recordings of 47 patients with generalized electrographic status epilepticus after cardiac arrest, five-minute epochs were selected every hour. Epochs were visually assessed and categorized into seven categories, including epileptiform discharges. Five quantitative measures were extracted, reflecting background continuity, discharge frequency, discharge periodicity, relative discharge power, and inter-discharge waveform correlation. The best achieved outcome within six months after cardiac arrest was categorized as 'good' (Cerebral Performance Category 1 to 2, i.e. no or moderate neurological disability) or 'poor' (CPC 3 to 5, i.e. severe disability, coma, or death).

Ten (22\%) patients had a good outcome. Status epilepticus in patients with good outcome started later (median 45 vs. 29 hours after cardiac arrest, $p<0.001$ ), more often ceased for at least 12 hours $(90 \%$ vs. $16 \%, p=0.02)$, and was less often treated with antiepileptic drugs ( $30 \%$ vs. $73 \%, p=0.02)$. Status epilepticus in patients with a good outcome always evolved from a continuous background pattern, as opposed to evolution from a discontinuous background pattern in 14 patients (38\%) with a poor outcome. Epileptiform patterns of patients with good outcome had higher background continuity ( 1.00 vs. $0.83, p<0.001$ ), higher discharge frequency ( 1.63 vs. $0.90 \mathrm{~Hz}, \mathrm{p}=0.002$ ), lower relative discharge power $(0.29$ vs. $0.40, p=0.01)$, and lower discharge periodicity ( 0.32 vs. $0.45, p=0.04)$.

We present electrographic characteristics of status epilepticus after cardiac arrest that can be used to identify patients with possible recovery. Our results indicate that restoration of continuous background activity before the onset of status epilepticus is essential for a good outcome. 


\subsection{Introduction}

Electrographic status epilepticus occurs in 10 to $35 \%$ of comatose survivors of cardiac arrest $^{1-6}$ and has a strong association with poor outcome ${ }^{1,3,5,7,8}$. Due to the lack of widely accepted diagnostic criteria, several electrographic abnormalities may qualify as status epilepticus in postanoxic encephalopathy. It is unclear whether these various patterns reflect various grades of encephalopathy and prognosis, and for which of these patterns treatment with anticonvulsants may improve patients' outcome ${ }^{9,10}$. Although these have not been investigated systematically, certain differences between electrographic seizure patterns of patients with good and poor outcome have been observed. For example, generalized periodic discharges (GPDs) on a completely suppressed background are strongly associated with a poor outcome ${ }^{11}$, whereas patients with GPDs on a continuous, normal amplitude background may have a chance to survive $^{12}$. Other possible determinants of poor outcome are based on case studies and include a very regular inter-discharge interval, a constant discharge morphology, high spatial synchronization, and high relative power in the discharges $8,11,13-15$. In few small case series of survivors, opposite characteristics were observed ${ }^{4,16}$.

Besides the seizure pattern itself, its temporal evolution may indicate possibility for recovery. Prolonged seizure duration is associated with a poor outcome in both convulsive and non-convulsive status epilepticus ${ }^{17}$. Evolution of status epilepticus from a burst-suppression background, without preceding resoration of continuity, always resulted in a poor outcome in an observational study in 26 patients with postanoxic encephalopathy, whereas from patients with status epilepticus evolving from a continuous background two survived ${ }^{5}$. Seizure patterns that eventually lead to poor outcome appear to have a poor electrographic response to antiepileptic treatment ${ }^{1,14}$. Possible explanations are twofold. On one hand, electrographic seizures not responding to therapy tend to continue and may lead to secondary neuronal damage. On the other hand, not responding to medication may indicate severe encephalopathy instead of a true epileptic, reversible phenomenon.

Visual analysis of EEG in critical care suffers from poor interobserver agreement, despite efforts to introduce unified criteria ${ }^{18}$. Quantitative EEG overcomes this problem, and helps to save time in assessment of lengthy continuous EEG recordings. Besides, quantitative EEG allows for statistical analysis, which is an advantage for research purposes.

We aim to identify and quantify EEG characteristics of generalized postanoxic status epilepticus that help to select patients with or without possible recovery. We focus on seizure patterns and their temporal evolution, and use quantitative EEG features that reflect key features used in visual interpretation. We hypothesize that seizure onset before improvement to a continuous background, the absence of background continuity during seizures, a low discharge frequency, high periodicity, and high correlation between consecutive discharges indicate a poor prognosis. 


\subsection{Methods}

\subsubsection{Patients}

Data were collected from two teaching hospitals in The Netherlands. Between July 2010 and September 2014, all subsequent comatose patients that were admitted to the Intensive Care Unit (ICU) after cardiac arrest were included in a prospective cohort study on the predictive value of cEEG on outcome ${ }^{6}$. The Medical Ethics Committee Twente approved the protocol and waived the need for informed consent for EEG monitoring during the ICU stay and clinical follow-up. Patients were included in the current analysis if the EEG showed generalized electrographic seizures during the recording on visual analysis. We defined electrographic seizures as generalized spike-wave discharges at $3 \mathrm{~Hz}$ or faster, or clearly evolving discharges of any type at $4 \mathrm{~Hz}$ or faster, in accordance with standardized Critical Care EEG terminology ${ }^{18}$. Also, generalized periodic discharges with a minimum frequency of $0.5 \mathrm{~Hz}$ were included. For continuous seizure activity, the minimum duration to qualify as status epilepticus was 30 minutes. Intermittent seizures of 5 minutes and longer, with maximum inter-seizure interval of 5 minutes were also included, if the total seizure duration was more than 30 minutes. Rhythmic delta activity or burst-suppression patterns with epileptiform burstshapes were not included. Patients were excluded from the analysis if cEEG started later than 24 hours after cardiac arrest or if they had any other concommittant severe brain injury (for example trauma, stroke).

\subsubsection{Outcome}

The primary outcome measure was the best achieved Cerebral Performance Category $(\mathrm{CPC}){ }^{19}$ score in the first six months after cardiac arrest, assessed by telephone interviews at three and six months. This ordinal scale ranges from 1 , indicating full recovery, to 5, indicating death. CPC scores were dichotomized into 'good' (CPC 1-2, i.e. no or moderate neurological disability) and 'poor' (CPC 3-5, i.e. severe disability, coma, or death).

Other prospectively collected data were: gender, age, results of clinical neurological evaluation (Glasgow Coma Scale scores, brain stem reflexes, myoclonus), serum lactate levels, median nerve somatosensory evoked potentials, all sedative medication, and medication for treatment of status epilepticus.

\subsubsection{EEG recordings}

EEG recordings were started as soon as possible within 24 hours after patients' admission to the ICU, and continued until patients were awake or until the decision to withdraw treatment was made, with a maximum of five days. Twenty-one silver/silver chloride cup electrodes were placed on the scalp according to the international 1020 system. Quantitative EEG analysis was performed offline. EEG data were not used for decisions regarding treatment withdrawal. However, treating physicians were not blinded to the EEG. Treatment of electrographic seizures was left to the discretion of the treating physician. 


\subsubsection{Selection of EEG epochs}

Five minute EEG epochs were selected automatically every hour during the complete recordings. A computer algorithm, as used in a previous quantitative EEG study ${ }^{20}$, was applied to select only epochs that were free of artifacts. A movement artifact parameter was calculated as the relative fraction of high voltage signal ( $>200 \mu \mathrm{V})$, a muscle artifact parameter as the power ratio between high $(25-40 \mathrm{~Hz})$ and low $(2-25 \mathrm{~Hz})$ frequencies, and a loose electrode artifact parameter as the relative amount of zero signal (with standard deviation lower than $1 \mu \mathrm{V}$ for at least 1 second). A global artifact parameter was calculated from these three measures. Data from 10 minutes before and 10 minutes after a selected time point were assessed and divided into segments of 30 seconds. The 10 consecutive segments with the lowest global artifact parameter were selected as final epoch. If the individual or global artifact parameters exceeded a predefined value, no epoch was selected for that hour. Before any further qualitative or quantitative analysis, all epochs were filtered by a zero-phase, sixth order Butterworth bandpass filter $(0.530 \mathrm{~Hz})$ and transformed to the longitudinal bipolar montage.

\subsubsection{Qualitative categorization of EEG epochs}

All epochs with raw EEG data were presented to a reviewer by the computer, in random order. The reviewer was blinded for the patients' clinical condition during the registration, the recording time of the epoch, and the patient's outcome. Epochs were visually inspected and placed in one the following categories: isoelectric, low voltage, burst-suppression, diffusely slowed, normal, or epileptiform. Isoelectric epochs were defined as epochs without visible EEG activity. Low voltage epochs were defined as epochs with visible EEG activity, but with all amplitudes below 20 $\mu \mathrm{V}$. Burst-suppression was defined as the presence of clear increases in amplitude (bursts), followed by interburst intervals of at least 1s with low-voltage activity (suppressions). Bursts were required to have EEG amplitudes $>20 \mu \mathrm{V}$, otherwise the epoch was categorized as low voltage. Burst-suppression was further subdivided into 'burst-suppression with identical bursts' and 'burst-suppression without identical bursts'. 'Burst-suppression with identical bursts' was defined as burst-suppression in which shapes of subsequent bursts are identical ${ }^{21}$. Diffusely slowed epochs were defined as epochs with normal amplitude $(>20 \mu \mathrm{V})$ for $>90 \%$ of the time and dominant frequency in the delta or theta range $(1-8 \mathrm{~Hz})$. An epoch was categorized as normal if amplitudes were higher than $20 \mu \mathrm{V}$ and the dominant frequency was $8 \mathrm{~Hz}$ or higher, irrespective of reactivity and anterior-posterior differentiation. Epileptiform epochs included electrographic seizures and GPDs. Electrographic seizures were defined as generalized spike-wave discharges or clearly evolving discharges of any type, without quantifiable inter-discharge interval. GPDs were defined as a bilateral synchronous repetitions of a waveform with relatively uniform morphology and duration with a quantifiable inter-discharge interval between consecutive waveforms and recurrence of the waveform at nearly regular intervals, with minimum frequency $0.5 \mathrm{~Hz}$. We did not analyze electrographic seizures and GPDs separately, as the distinction between the two is often arbitrary in postanoxic encephalopathy. The visual analysis resulted in a time line of the temporal evolution of the EEG for every patient. The first epileptiform epoch on this time line defined the onset time of status epilepticus. As a measure for the tendency of epileptic discharges to disappear, we determined whether or 
not epileptiform discharges ceased for at least 12 consecutive hours. We did not analyze the duration of status epilepticus, as we considered this problematic. First, epileptiform discharges might reappear after a seizure-free interval. Second, because of the decision to withdraw treatment, in many cases EEG recordings were terminated during ongoing status epilepticus. To indicate the reliability of the visual assessment, a random set consisting of $15 \%$ of all epochs were assessed by a second reviewer using the same protocol.

\subsubsection{Quantitative EEG analysis}

Quantitative EEG analysis and statistical analysis were performed with MATLAB (MATLAB and Statistics Toolbox Release R2013b, The MathWorks, Inc., Natick, Massachusetts, United States).

\section{Assessment of background continuity}

A background continuity parameter was applied to all epochs to assess evolution of background continuity and to characterize epileptiform EEG patterns. The background continuity parameter was defined as follows:

$$
C=\frac{\mathrm{T}_{\text {norm }}}{\mathrm{T}_{\text {norm }}+\mathrm{T}_{\text {supp }}},
$$

where $\mathrm{T}_{\text {norm }}$ is the amount of time for which the EEG has amplitude $10 \mu \mathrm{V}$, and $\mathrm{T}_{\text {supp }}$ the amount of time for which the EEG is suppressed $(<10 \mu \mathrm{V})$, with a minimum suppression duration of $0.5 \mathrm{~s}$. In fact, this continuity parameter is the inverse of the burstsuppression ratio ${ }^{22}$. This calculation was performed for all bipolar derivations individually and then averaged. The continuity index equals 0 for low-voltage or isoelectric EEG and 1 for continuous EEG activity. Burst-suppression patterns patterns have values between 0 and 1 . For the assessment of background continuity evolution in relation to the onset time of status epilepticus, epochs were classified as continuous if the continuity index exceeded 0.9 . This indicates that we accepted a maximum suppression time of $10 \%$ resulting from spontaneous fluctuations.

\section{Quantitative analysis of epileptiform patterns}

All EEG epochs that were marked as epileptiform during visual assessment were further analyzed with regard to four quantitative EEG measures, all closely related to visual observations: discharge frequency, discharge periodicity, relative discharge power, and discharge correlation.. Calculations were performed per epoch and then averaged over all epileptiform epochs per patient.

All measures started with a detection algorithm for generalized epileptiform discharges. This algorithm is based on a method for detecting spike trains in neonatal seizures $^{23}$, and is especially useful for detecting epileptiform discharges with a quantifiable inter-discharge interval, as is the case for GPDs. It consists of a nonlinear energy operator to enhance signal with high amplitude and high frequency,defined as

$$
\phi(n)=\left|\left(x_{n-1} \cdot x_{n-2}\right)-\left(x_{n} \cdot x_{n-3}\right)\right|
$$


where $\phi(n)$ denotes the filtered signal, $x_{n}$ the current sample of signal $x, x_{n-1}$ the first sample before $x_{n}$, and so on. On the output of the nonlinear energy operator, we applied a moving average filter with a window size of $120 \mathrm{~ms}$. Next, the signal was divided into epochs of 5 seconds, with overlap of 4 seconds. To each epoch, an adaptive threshold was applied that was defined as

$$
T=0.6 \cdot\left[\sigma+q_{3}\right]
$$

with $\sigma$ and $q_{3}$ denoting the standard deviation and 75th percentile of the epoch, respectively. Unlike Deburchgraeve et al., we did not apply an additional 'spikiness criterion' to further reduce the number the high-energetic segments. This was left out, because periodic discharges in postanoxic encephalopathy often have sharp instead of spiky waveforms ${ }^{18}$, and the 'spikiness criterion' would exclude these types of discharges. If, after applying the adaptive threshold, the remaining high-energy segments are present in nine or more bipolar derivations at the same time, they were considered as 'generalized discharges'. As most epileptiform discharges in postanoxic encephalopathy are generalized, we accepted lateralized periodic discharges not being detected. The minimum required duration of discharges was $60 \mathrm{~ms}$ and the minimum interval between subsequent discharges was $200 \mathrm{~ms}$. The maximum duration of discharges was $0.5 \mathrm{~s}$, because otherwise they would qualify as bursts, by definition ${ }^{18}$. A minimum amplitude for discharges of $20 \mu \mathrm{V}$ was chosen to avoid electrocardiography (ECG) artifacts from being detected as epileptic discharges. The four features extracted are listed and explained in Table 4.1.

\subsubsection{Statistical analysis}

To test for differences in categorical variables between patients with good and poor outcome, we applied the Chi-square test or Fisher's exact test, depending on the distribution. To test between-group differences for continuous variables, we applied Student's t-tests or Mann-Whitney U tests, where applicable. Agreement between the two observers in visual categorization of EEG epochs was tested with Cohen's Kappa. All statistical testing was two-tailed and P-values $\leq 0.05$ were considered statistically significant.

\subsection{Results}

\subsubsection{Patient characteristics}

Of 288 patients that were monitored with cEEG after cardiac arrest, 47 had generalized electrographic status epilepticus and met the criteria for inclusion in this study. Patient characteristics are listed in Table 4.2, grouped by outcome. Patients with poor outcome did not differ from patients with good outcome in terms of age, gender, lactate level, timing and duration of cEEG recording, resuscitation characteristics, and sedation requirements. None of the patients with good outcome had a GCS-score of 3 or absent pupillary light reflexes after 72 hours. Myoclonus was observed in a majority (82\%) of patients with poor outcome in the first 72 hours after cardiac arrest, while only one patients with good outcome had myoclonus. A majority of patients was treated with antiepileptic drugs. More patients with poor outcome were treated ( 73 vs. $30 \%, P=0.02$ ) 
Table 4.1: Definitions of quantitative EEG features, extracted after applying the epileptiform discharge detection algorithm.

\begin{tabular}{|c|c|}
\hline Quantitative feature & Definition \\
\hline Discharge frequency & $\begin{array}{l}1 / \text { (median inter-discharge interval). The inter- } \\
\text { discharge interval is the difference in onset time } \\
\text { between two consecutive generalized discharge } \\
\text { segments. }\end{array}$ \\
\hline Relative discharge power & $\begin{array}{l}\text { The summed power in generalized discharge seg- } \\
\text { ments, divided by the total signal power. Calculated } \\
\text { for each channel individually and then averaged over } \\
\text { channels. Power was denoted as the square of the } \\
\text { signal amplitude, averaged over time. Value [0-1]. }\end{array}$ \\
\hline Discharge periodicity & $\begin{array}{l}\text { Fraction of all inter-discharge intervals whose dura- } \\
\text { tion is no more than } 25 \% \text { shorter or longer than the } \\
\text { median inter-discharge interval. This feature was } \\
\text { only calculated if the discharge frequency was higher } \\
\text { than } 0.2 \mathrm{~Hz} \text {. Value [0-1]. }\end{array}$ \\
\hline Discharge correlation & $\begin{array}{l}\text { The average cross-correlation coefficient for each } \\
\text { generalized discharge with its } 10 \text { preceding general- } \\
\text { ized discharges. Calculated for each channel individ- } \\
\text { ually and then averaged over channels. Value [0-1]. } \\
\text { This feature was only calculated if the discharge fre- } \\
\text { quency was higher than } 0.2 \mathrm{~Hz} \text {. }\end{array}$ \\
\hline
\end{tabular}


and if so, more different anti-epileptic drugs were used, as compared with patients with a good outcome.

\subsubsection{Qualitative assessment of EEG epochs}

In total, 3306 epochs were assessed by visual inspection. Of these epochs, 500 were assessed by a second reviewer. Cohen's Kappa was 0.74 for the distinction between epileptiform and non-epileptiform epochs.

\subsubsection{Temporal evolution of electrographic status epilepticus}

Figures 4.1 and 4.2 show typical examples of the EEG evolution in patients with a poor and a good outcome, respectively. The patient in Figure 4.1 develops status epilepticus from a burst-suppression pattern, without preceding improvement to a continuous background. This is reflected by both the qualitative assessment and the quantitative continuity parameter. Note that background continuity improves with time, but never reaches values higher than 0.9 . Improvement of continuity is only caused by the increase in discharge frequency. Figure 4.2 shows the EEG evolution for a patient with good outcome. Note that, in contrast to Figure 4.1, the EEG readily improves to a continuous background, and remains continuous thereafter. Epileptiform discharges appear after background continuity has been reached. More clearly than for the patient in Figure 4.1, discharges appear shortly after tapering sedative medication, and disappear quickly after the administration of an anti-epileptic drug (phenytoin). The relative contribution of the discharges to the total signal power in this patient is low throughout time.

The upper part of Table 4.3 summarizes characteristics of the temporal evolution of electrographic status epilepticus for patients with poor and good outcome. All patients with good outcome improved towards a continuous EEG, defined as a background continuity parameter higher than 0.9 , before the onset of status epilepticus as compared with $62 \%$ of patients with a poor outcome. Only $84 \%$ of patients with poor outcome ever improved to a continuous EEG. The median onset time of status epilepticus was 29 hours after cardiac arrest for patients with poor outcome, and 45 hours for patients with good outcome $(P<0.001)$. In patients with a good outcome, status epilepticus never started within 39 hours after cardiac arrest. Epileptiform discharges we more likely to cease for at least 12 hours in patients with good outcome ( $90 \%$ vs. $16 \%, p=0.02)$. In $70 \%$ of patients with poor outcome, status epilepticus was preceded by burst-suppression with identical bursts as compared with none of the patients with a good outcome $(\mathrm{P}<0.001)$.

\subsubsection{Quantitative characterization of epileptiform epochs}

Figure 4.3 shows two typical examples of observed EEG patterns, the result of the discharge detection algorithm, and the extracted quantitative EEG features. Figure $4.3 \mathrm{~A}$ is a pattern that was observed in a patient with poor outcome. It shows generalized periodic discharges at regular intervals, with constant discharge morphology, high spatial synchronization, relative high power in the discharges, and a suppressed background, reflected by the extracted quantitative EEG features. The pattern in Figure 4.3B was observed in a patient with good outcome. It consists of periodic discharges that wax 
Table 4.2: Patient characteristics, grouped by outcome.

\begin{tabular}{|c|c|c|c|}
\hline & $\begin{array}{l}\text { Poor outcome } \\
\text { (CPC 3-5) }\end{array}$ & $\begin{array}{l}\text { Good outcome } \\
\text { (CPC 1-2) }\end{array}$ & P-value \\
\hline Number of patients & 37 & 10 & \\
\hline Male & $23(62 \%)$ & $7(70 \%)$ & 0.727 \\
\hline Age & $68(27-82)$ & $68(51-88)$ & 0.848 \\
\hline Witnessed cardiac arrest & $25(68 \%)$ & $7(70 \%)$ & 1.000 \\
\hline Resuscitation outside hospital & $33(89 \%)$ & $9(90 \%)$ & 1.000 \\
\hline Initial rhythm VT/VF & $19(51 \%)$ & $9(90 \%)$ & 0.083 \\
\hline No. of shocks & $1(0-16)$ & $2(0-5)$ & 0.434 \\
\hline Cause of non-cardiac origin & $9(24 \%)$ & $1(10 \%)$ & 0.617 \\
\hline Lactate level $(\mathrm{mmol} / \mathrm{L})$ & $2.5(0.6-9.7)$ & $1.5(0.4-4.6)$ & 0.177 \\
\hline Therapeutic hypothermia $\left(33^{\circ} \mathrm{C}\right)$ & $36(97 \%)$ & $9(90 \%)$ & 0.348 \\
\hline Sedation with propofol & $34(92 \%)$ & $10(100 \%)$ & 1.000 \\
\hline Maximum dose (mg/kg/h) & $2.7(1.5-5.3)$ & $3.6(1.7-4.3)$ & 0.130 \\
\hline Sedation with midazolam & $16(43 \%)$ & $5(50 \%)$ & 0.734 \\
\hline Maximum dose (mg/kg/h) & $0.14(0.03-0.89)$ & $0.14(0.06-0.36)$ & 0.924 \\
\hline Sedation with fentanyl & $19(51 \%)$ & $7(70 \%)$ & 0.475 \\
\hline Maximum dose $(\mu \mathrm{g} / \mathrm{kg} / \mathrm{h})$ & $1.7(1.0-2.9)$ & $1.8(1.3-2.6)$ & 0.497 \\
\hline Sedation with remifentanyl & $7(19 \%)$ & $0(0 \%)$ & 0.318 \\
\hline Maximum dose $(\mu \mathrm{g} / \mathrm{kg} / \mathrm{h})$ & $6.3(2.3-13)$ & & \\
\hline Sedation with morphine & $9(24 \%)$ & $2(20 \%)$ & 1.000 \\
\hline Maximum dose (mg/kg/h) & $0.27(0.20-0.65)$ & $0.40(0.22-0.58)$ & 0.667 \\
\hline Treatment with AEDs & $27(73 \%)$ & $3(30 \%)$ & 0.023 \\
\hline Number of AEDs & $1(0-3)$ & $0(0-2)$ & 0.040 \\
\hline Treatment with phenytoin & $17(46 \%)$ & $1(10 \%)$ & 0.065 \\
\hline Treatment with levetiracetam & $8(22 \%)$ & $0(0 \%)$ & 0.147 \\
\hline Treatment with valproic acid & $11(30 \%)$ & $3(30 \%)$ & 1.000 \\
\hline cEEG start time (hours) & $13(3-24)$ & $7(2-17)$ & 0.175 \\
\hline cEEG duration (hours) & $66(20-217)$ & $68(59-166)$ & 0.434 \\
\hline $\mathrm{GCS} \geq \mathrm{E} 1 \mathrm{M} 2$ at $72 \mathrm{~h}$ & $7(19 \%)$ & $10(100 \%)$ & $<0.001$ \\
\hline Present pupillary light reflexes at $72 \mathrm{~h}$ & $33(89 \%)$ & $10(100 \%)$ & 0.113 \\
\hline Bilateral absent $\mathrm{N} 20$ response & $16(47 \%)$ & $0(0 \%)$ & 0.066 \\
\hline Myoclonus observed within $72 \mathrm{~h}$ & $32(87 \%)$ & $1(10 \%)$ & $<0.001$ \\
\hline
\end{tabular}

Numbers given as median (range) unless otherwise indicated. AEDs: Antiepileptic drugs; GCS: Glasgow Coma Scale score; VF: Ventricular Fibrillation; VT: Ventricular Tachycardia. 
A

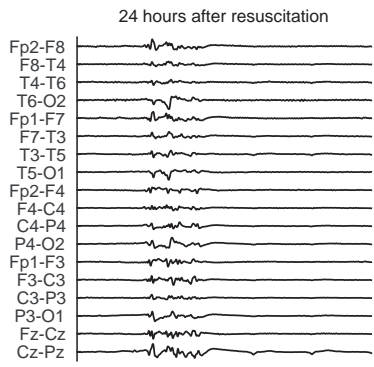

49 hours after resuscitation

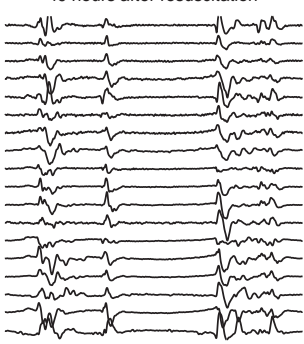

B

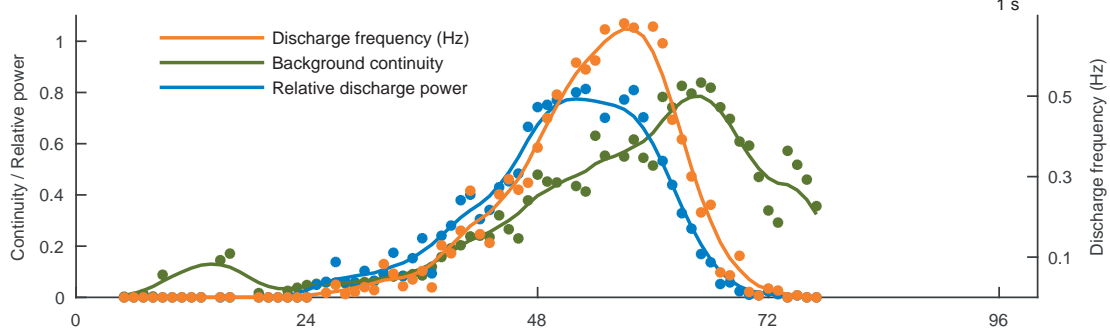

C

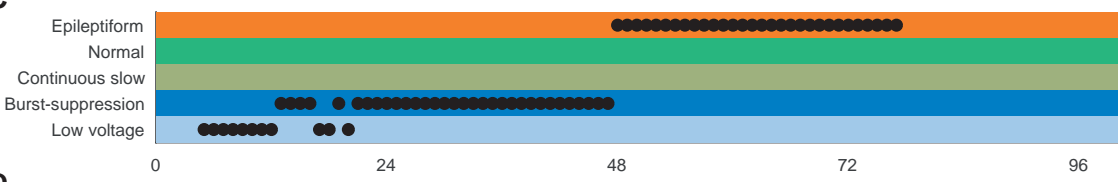

。

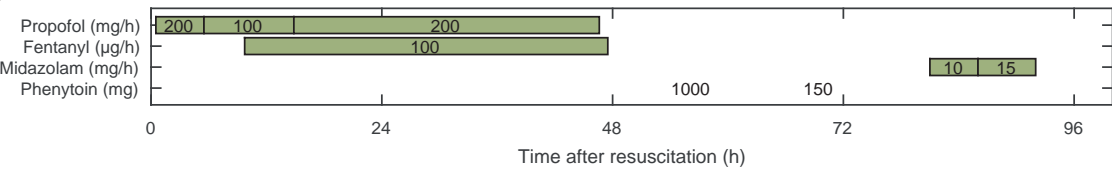

Figure 4.1: Temporal EEG evolution of a patient with poor outcome. A: Three samples of EEG at different time intervals. B: Evolution of background continuity, discharge frequency, and relative discharge power over time. The dots represent the actual values, the line results from subsequently applying linear interpolation and a moving average filter with window length 5 hours. C: Results of categorization after visual interpretation. D: administered sedatives and antiepileptic drugs. Note: between 68 and 77 hours, the detected discharge frequency drops suddenly. This is caused by the fact that, during these hours, the amplitude and spatial generalization of discharges had fallen below the detection threshold. 
A

C

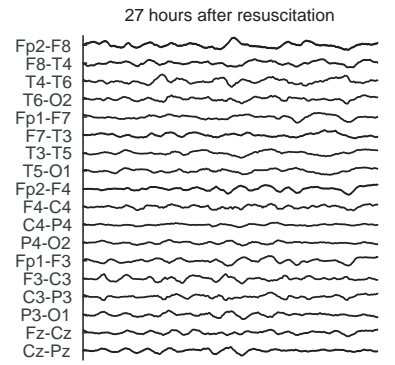

B
48 hours after resuscitation

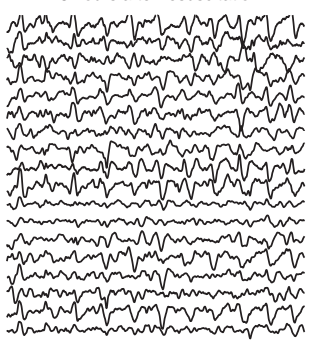

67 hours after resuscitation

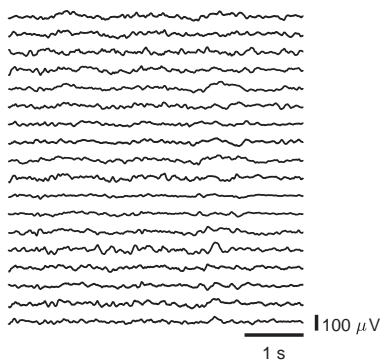

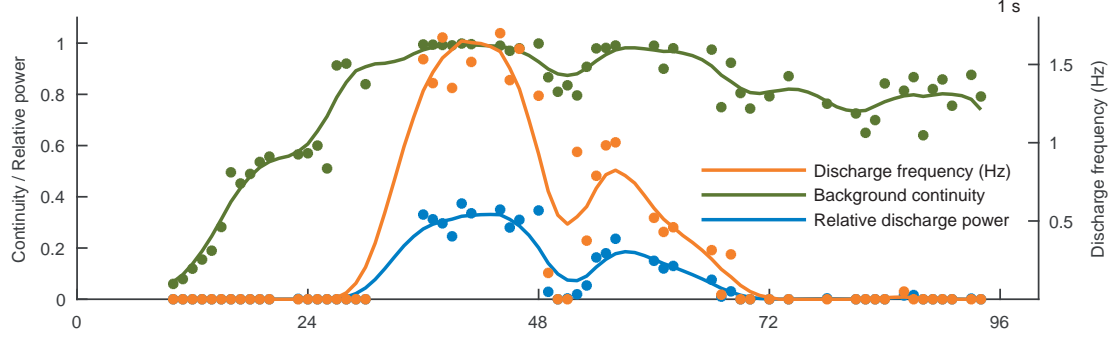

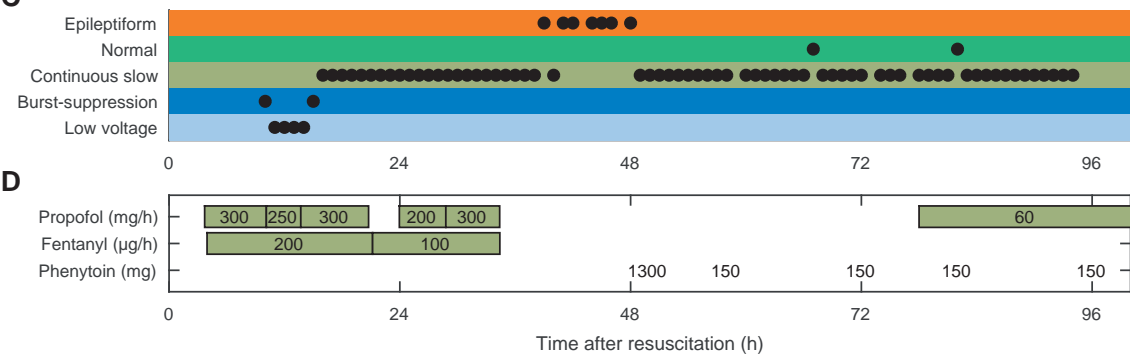

Figure 4.2: Temporal EEG evolution of a patient with good outcome. A: EEG samples at three instances of time. B: Evolution of background continuity, discharge frequency, and relative discharge power over time. The dots represent the actual values, the line results from subsequently applying linear interpolation and a moving average filter with window length 5 hours. C: Results of categorization after visual interpretation. D: Administered sedatives and antiepileptic drugs. 
Table 4.3: Evolution of electrographic status epilepticus and quantitative EEG features of epileptiform epochs, grouped by outcome.

\begin{tabular}{lccc}
\hline & $\begin{array}{c}\text { Poor outcome } \\
\text { (CPC 3-5) } \\
\mathrm{N}=37\end{array}$ & $\begin{array}{c}\text { Good outcome } \\
\text { (CPC 1-2) } \\
\mathrm{N}=10\end{array}$ & $\mathrm{P}$ \\
\hline Evolution of status epilepticus & & & \\
$\quad$ Background continuity $>0.9$ before SE & $23(62 \%)$ & $10(100 \%)$ & 0.02 \\
Background continuity never $>0.9$ & $6(16 \%)$ & $0(0 \%)$ & 0.22 \\
Time to onset of SE (hours) & $29(13-56)$ & $45(39-62)$ & $<0001$ \\
Cessation of SE for $\geq 12 \mathrm{~h}$ & $6(16 \%)$ & $9(90 \%)$ & 0.02 \\
SE preceded by identical bursts & $26(70 \%)$ & $0(0 \%)$ & $<0001$ \\
& & & \\
qEEG features of epileptiform epochs & & & \\
Background continuity & $0.83(0.43-1.00)$ & $1.00(0.92-1.00)$ & $<0001$ \\
Discharge frequency & $0.90(0.26-1.75)$ & $1.63(0.82-2.29)$ & 0.002 \\
Relative discharge power & $0.40(0.16-0.80)$ & $0.29(0.17-0.42)$ & 0.01 \\
Discharge periodicity & $0.45(0.08-0.85)$ & $0.32(0.20-0.57)$ & 0.04 \\
Discharge correlation & $0.66(0.30-0.87)$ & $0.59(0.30-0.64)$ & 0.16 \\
\hline
\end{tabular}

Numbers given as median (range) unless otherwise indicated. CPC: Cerebral Performance Category; SE: status epilepticus; qEEG: quantitative electroencephalography.

and wane, with evolving discharge morphology on a continuous, normal voltage background pattern. The inter-discharge intervals are more variable, and spatial generalization is less obvious as compared with the pattern in Figure 4.3A. One can loosely state that pattern 4.3A is less 'complex' and more 'predictable' than pattern 4.3B.

In general, the discharge detection algorithm worked well, as illustrated in Figure 4.3. If we would define epochs as epileptiform if the detected discharge frequency was 0.5 $\mathrm{Hz}$ or higher, $78 \%$ of 3306 epochs would be classified correctly, as compared with visual categorization. In the lower part of Table 4.3, comparisons of quantitative EEG features of epileptiform epochs of patients with poor and good outcome are presented. Epileptiform epochs of patients with good outcome had higher background continuity (1.00 vs. $0.83, p<0.001$ ), higher discharge frequency (1.63 vs. $0.90 \mathrm{~Hz}, \mathrm{p}=0.002)$, lower relative discharge power $(0.29$ vs. $0.40, p=0.01)$, and lower discharge periodicity $(0.32$ vs. $0.45, \mathrm{p}=0.04)$. Mean background continuity was never below 0.92 and mean discharge frequency was never below $0.82 \mathrm{~Hz}$ in patients with a good outcome. There was no statistically significant difference between patients with poor and good outcome in correlation between subsequent discharge waveforms ( 0.59 vs. $0.66, p=0.16)$.

\subsection{Discussion}

In this study, we present quantitative EEG features of generalized status epilepticus in postanoxic encephalopathy in relation to possible recovery. While other studies relied on qualitative descriptions, quantitative EEG features overcome problems with interobserver agreement and allow for statistical analysis. We introduce five quantitative 
A

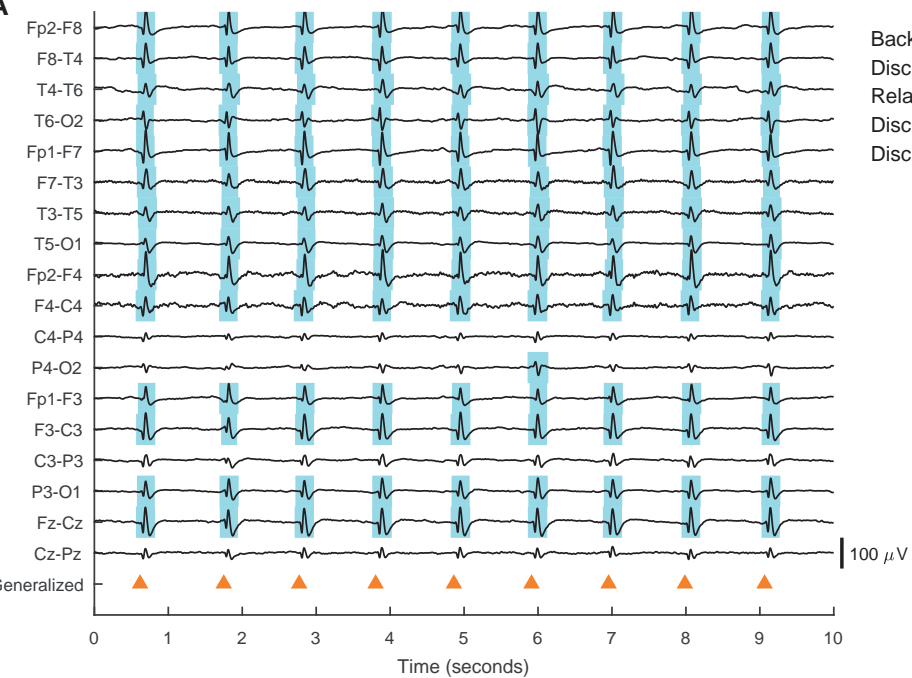

B

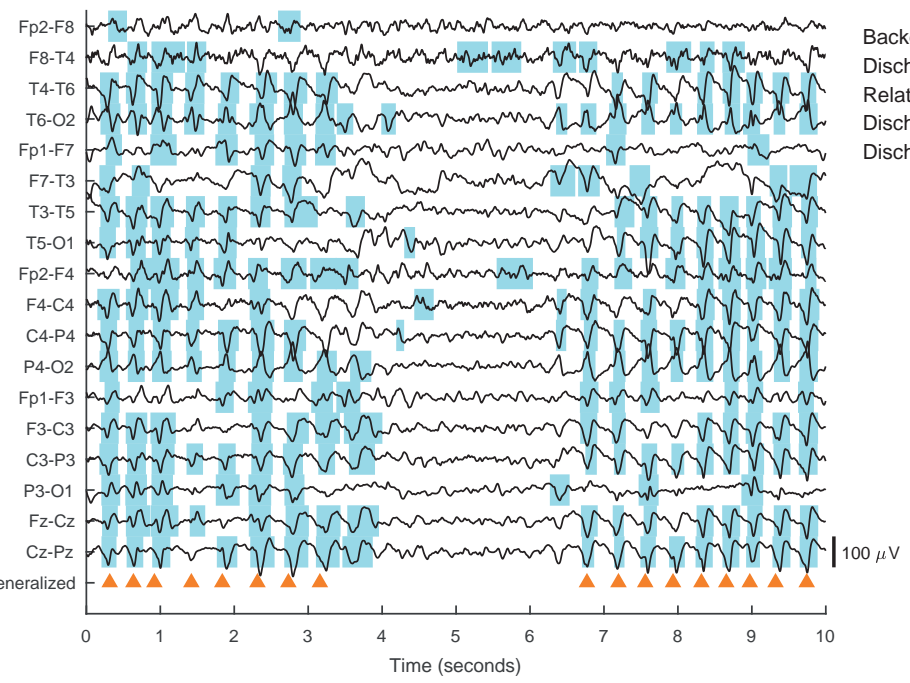

Background continuity $\quad 1.00$ Discharge frequency $(\mathrm{Hz}) \quad 2.16$ Relative discharge power $\quad 0.29$ Discharge periodicity $\quad 0.52$ Discharge correlation $\quad 0.54$

Figure 4.3: Two examples of electrographic seizure patterns, as observed in study subjects. High-energetic segments selected by the detection algorithm are highlighted. Orange triangles at the bottom indicate when a generalized discharge is detected. A: Typical example of electrographic seizure pattern as recorded from a patient with poor outcome. The pattern consists of generalized periodic discharges at regular intervals, with a constant morphology, high spatial synchronization, relative high power in the discharges, on a suppressed background. Note that all high-energetic segments are part of a generalized discharge. B: Typical example of electrographic seizure pattern as recorded from a patient with good outcome. It consists of discharges that show evolving discharge morphology on a continuous, normal voltage background, where the inter-discharge intervals are more variable, and spatial generalization is less obvious as compared with the pattern in panel A. Note that not all selected high-energy segments are discharges, and discharges with a low amplitude $(<20 \mu \mathrm{V})$ are not detected. 
EEG features closely related to aspects of visual assessment, which helps translating our results to clinical practice.

Unlike patients with a poor outcome, all patients with a good outcome improved towards a continuous EEG background pattern before the onset of status epilepticus. Outcome was always poor if status epilepticus developed during the first 36 hours after cardiac arrest, the period with targeted temperature management and the associated sedative medication. Outcome was also invariably poor if electrographic status epilepticus was preceded by burst suppression with identical bursts. Seizure patterns of patients with good outcome showed higher background continuity, higher epileptic discharge frequency, lower relative discharge power, and lower discharge periodicity as compared with patients with poor outcome. Outcome was invariably poor when more than two antiepileptic drugs were needed to suppress seizure activity.

As compared with previous literature, the incidence of electrographic status epilepticus was high in our cohort. Also, relatively many patients had a good outcome. In previous studies, the diagnosis of status epilepticus was often based on repetitive routine EEGs, typically applied later than 72 hours after cardiac arrest, and the decision to perform EEG was often based on the presence of myoclonus ${ }^{3,4,8}$. In our study, electrographic seizure patterns leading to poor outcome had a longer duration and were more often accompanied by myoclonus, as compared with seizure patterns of patients with good outcome. Therefore, previous case series may have included a subsample of patients with a high probability of poor outcome. Early, self-limiting status epilepticus with a good outcome was probably not diagnosed. Another explanation for the relatively large fraction of patients with good outcome is the broad range of EEG patterns included in our study, as compared to previous work.

None of the 14 patients who developed status epilepticus before improvement to a continuous pattern survived. Moreover, $16 \%$ of patients with a poor outcome never improved towards a continuous background pattern. These results are in agreement with previous findings in a smaller case series of postanoxic status epilepticus ${ }^{5}$ and with our own findings in an unselected population of postanoxic encephalopathy ${ }^{6}$. Apparently, early improvement towards a continuous background EEG is a key feature of recovery from postanoxic encephalopathy.

We chose to characterize electrographic seizure patterns by quantitative EEG features that closely resemble features assessed by visual analysis. Accordingly, the results can be used in clinical practice, where visual assessment is the gold standard and real time quantitative EEG is often not available. However, for clinical decision making, differences between groups of patients with good and poor outcome should ideally be translated into predictive values for individual patients. This would require a clear definition of threshold values, to be confirmed in a separate test set. Seizure patterns of patients with a poor outcome differed from those with good outcome quantitatively, with more background suppression, lower discharge frequency, and higher periodicity of discharges. These findings demonstrate that the damaged neural networks underlying electrographic seizures have a lower complexity in patients with poor outcome as compared with patients with good outcome. A lower network complexity reflects a greater extent of ischemic damage, indicating that, especially in patients with a poor outcome, 
'epileptiform' patterns are an expression of severe ischemic damage, rather than true epilepsy, which is potentially reversible by treatment with antiepileptic drugs. In a computer model, we have demonstrated that generalized periodic discharges may result from selective damage to excitatory synapses to inhibitory interneurons ${ }^{9}$, disrupting the essential balance between excitatory and inhibitory input in this microcircuit ${ }^{24}$. We speculate that in some patients this failure of excitatory input to inhibitory interneurons is irreversible; such persistent failure of synaptic transmission has indeed been suggested by Bolay and others ${ }^{25,26}$.

Patients with electrographic status epilepticus and poor outcome were more often treated with antiepileptic drugs and, if so, with more different drugs than patients with a good outcome. Still, seizures did not disappear in the majority of these patients. Many physicians treat electrographic status epilepticus after cardiac arrest, despite lack of evidence for a beneficial effect on patients' outcome. However, only one third of epilepsy experts treats these patients equally intensive as patients with overt status epilepticus ${ }^{27}$. Paradoxically, for most neurologists the threshold to treat patients with overt myoclonus is lower than for patients with non-convulsive electroencephalographic seizures, whereas irreversible damage and a poor outcome are more likely in patients with myoclonus ${ }^{10}$. The effect of intensive antiepileptic treatment is currently under investigation in a randomized multicenter trial (NCT02056236) ${ }^{28}$.

This study has limitations. First, all our findings are based on 5 minute epochs that were automatically selected every hour during the first 72 hours after cardiac arrest. Recurrent seizures lasting less than 60 minutes may therefore have been missed. However, epileptiform discharges in postanoxic encephalopathy usually do not disappear spontaneously within hours and will therefore most likely be detected, even using our limited temporal sampling. Second, the discharge detection algorithm was unable to classify $22 \%$ of all epochs correcly, as compared to visual analysis. However, our algorithm performed equally well as previously published seizure detection algorithms ${ }^{23,29,30}$, despite heterogenity in discharge waveforms and background patterns. We considered the algorithm sufficiently reliable for adequate quantification and comparison of the studied parameters. Finally, our results may have been influenced by sedative agents. For example, propofol may induce discontinuities and - with high doses burst-suppression patterns. However, in healthy brains, with the dosages that were used in our patients, the EEG remains continuous, with anteriorization of the alpha rhythm ${ }^{31}$. Moreover, maximum propofol doses were not significantly different in both outcome groups.

\section{References}

1. Legriel S, Bruneel F, Sediri H, Hilly J, Abbosh N, Lagarrigue MH, et al. Early EEG monitoring for detecting postanoxic status epilepticus during therapeutic hypothermia: a pilot study. Neurocritical Care 2009; 11: 338-344.

2. Rittenberger JC, Popescu A, Brenner RP, Guyette FX, Callaway CW. Frequency and timing of nonconvulsive status epilepticus in comatose post-cardiac arrest subjects treated with hypothermia. Neurocritical Care 2012; 16: 114-122.

3. Rossetti AO, Logroscino G, Liaudet L, Ruffieux C, Ribordy V, Schaller MD, et al. Status epilepticus: An independent outcome predictor after cerebral anoxia. Neurology 2007; 69: 255-260. 
4. Rossetti AO, Oddo M, Liaudet L, Kaplan PW. Predictors of awakening from postanoxic status epilepticus after therapeutic hypothermia. Neurology 2009; 72: 744-749.

5. Rundgren M, Westhall E, Cronberg T, Rosén I, Friberg H. Continuous amplitude-integrated electroencephalogram predicts outcome in hypothermia-treated cardiac arrest patients. Critical Care Medicine 2010; 38: 1838-1844.

6. Tjepkema-Cloostermans MC, Hofmeijer J, Trof RJ, Blans MJ, Beishuizen A, van Putten MJAM. Electroencephalogram predicts outcome in patients with postanoxic coma during mild therapeutic hypothermia. Critical Care Medicine 2015; 43: 159-167.

7. Hofmeijer J, Tjepkema-Cloostermans MC, Blans MJ, Beishuizen A, van Putten MJAM. Unstandardized Treatment of Electroencephalographic Status Epilepticus Does Not Improve Outcome of Comatose Patients after Cardiac Arrest. Frontiers in Neurology 2014; 5: 39.

8. San-Juan OD, Chiappa KH, Costello DJ, Cole AJ. Periodic epileptiform discharges in hypoxic encephalopathy: BiPLEDs and GPEDs as a poor prognosis for survival. Seizure 2009; 18: 365-368.

9. Tjepkema-Cloostermans MC, Hindriks R, Hofmeijer J, van Putten MJAM. Generalized periodic discharges after acute cerebral ischemia: Reflection of selective synaptic failure? Clinical Neurophysiology 2014; 125: 255-262.

10. Young GB, Gilbert JJ, Zochodne DW. The significance of myoclonic status epilepticus in postanoxic coma. Neurology 1990; 40: 1843-1848.

11. Bauer G, Trinka E, Kaplan PW. EEG Patterns in Hypoxic Encephalopathies (Post-Cardiac Arrest Syndrome). Journal of Clinical Neurophysiology 2013; 30: 477-489.

12. Milani P, Malissin I, Tran-Dinh YR, Deye N, Baud F, Lévy BI, et al. Prognostic EEG patterns in patients resuscitated from cardiac arrest with particular focus on Generalized Periodic Epileptiform Discharges (GPEDs). Neurophysiologie Clinique 2014; 44: 153-164.

13. Celesia GG, Grigg MM, Ross E. Generalized status myoclonicus in acute anoxic and toxicmetabolic encephalopathies. Archives of Neurology 1988; 45: 781-784.

14. Friberg H, Westhall E, Rosén I, Rundgren M, Nielsen N, Cronberg T. Clinical review: Continuous and simplified electroencephalography to monitor brain recovery after cardiac arrest. Critical Care 2013; 17: 233.

15. Thömke F, Marx JJ, Sauer O, Hundsberger T, Hägele S, Wiechelt J, et al. Observations on comatose survivors of cardiopulmonary resuscitation with generalized myoclonus. BMC neurology 2005; 5: 14.

16. Hovland A, Nielsen EW, Klüver J, Salvesen R. EEG should be performed during induced hypothermia. Resuscitation 2006; 68: 143-6.

17. Young GB, Claassen J. Nonconvulsive status epilepticus and brain damage: further evidence, more questions. Neurology 2010; 75: 760-1.

18. Hirsch LJ, LaRoche SM, Gaspard N, Gerard E, Svoronos A, Herman ST, et al. American Clinical Neurophysiology Society's Standardized Critical Care EEG Terminology. Journal of Clinical Neurophysiology 2013; 30: 1-27.

19. Jennett $B$, Bond M. Assessment of outcome after severe brain damage: A practical scale. The Lancet 1975; 305: 480-484.

20. Tjepkema-Cloostermans MC, van Meulen FB, Meinsma G, Van Putten MJAM. A Cerebral Recovery Index (CRI) for early prognosis in patients after cardiac arrest. Critical Care 2013; 17: R252.

21. Hofmeijer J, Tjepkema-Cloostermans MC, van Putten MJAM. Burst-suppression with identical bursts: A distinct EEG pattern with poor outcome in postanoxic coma. Clinical Neurophysiology 2014; 125: 947-954.

22. Rampil IJ, Weiskopf RB, Brown JG, Eger El, Johnson BH, Holmes MA, et al. 1653 and isoflurane produce similar dose-related changes in the electroencephalogram of pigs. Anesthesiology 1988; 69: 298-302. 
23. Deburchgraeve W, Cherian PJ, De Vos M, Swarte RM, Blok JH, Visser GH, et al. Automated neonatal seizure detection mimicking a human observer reading EEG. Clinical Neurophysiology 2008; 119: 2447-2454.

24. Paz JT, Huguenard JR. Microcircuits and their interactions in epilepsy: is the focus out of focus? Nature Neuroscience 2015; 18: 351-359.

25. Bolay H, Gursoy-Ozdemir Y, Sara Y, Onur R, Can A, Dalkara T. Persistent Defect in Transmitter Release and Synapsin Phosphorylation in Cerebral Cortex After Transient Moderate Ischemic Injury. Stroke 2002; 33: 1369-1375.

26. Hofmeijer J, Mulder ATB, Farinha AC, Van Putten MJAM, Le Feber J. Mild hypoxia affects synaptic connectivity in cultured neuronal networks. Brain Research 2014; 1557: 180-189.

27. Bouwes A, Kuiper MA, Hijdra A, Horn J. Induced hypothermia and determination of neurological outcome after CPR in ICUs in the Netherlands: results of a survey. Resuscitation 2010; 81: 393-397.

28. Ruijter BJ, van Putten MJ, Horn J, Blans MJ, Beishuizen A, van Rootselaar AF, et al. Treatment of electroencephalographic status epilepticus after cardiopulmonary resuscitation (TELSTAR): study protocol for a randomized controlled trial. Trials 2014; 15: 433.

29. van Putten MJAM, Kind T, Visser F, Lagerburg V. Detecting temporal lobe seizures from scalp EEG recordings: A comparison of various features. Clinical Neurophysiology 2005; 116: 24802489.

30. Sierra-Marcos A, Scheuer ML, Rossetti AO. Seizure detection with automated EEG analysis: A validation study focusing on periodic patterns. Clinical Neurophysiology 2014; 126: 456-462.

31. Hindriks R, van Putten MJaM. Meanfield modeling of propofol-induced changes in spontaneous EEG rhythms. Neurolmage 2012; 60: 2323-2334. 


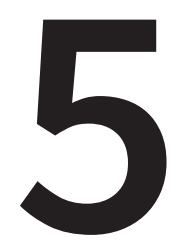

\section{Propofol does not affect the} reliability of early EEG for outcome prediction of comatose patients after cardiac arrest 


\section{Abstract}

Objective

Methods

Results

Conclusions

During the first 24h after cardiac arrest, EEG is a sensitive tool for the prediction of neurological outcome. In this period, patients usually receive sedative medication, such as propofol. We aim to quantify the effects of propofol on the postanoxic EEG and to assess their consequences for the reliability of outcome predictions.

In a prospective cohort study on postanoxic coma, we analyzed the EEG within the first $72 \mathrm{~h}$ after cardiac arrest. At six time points, EEG patterns were classified as favorable (continuous), unfavorable (generalized suppression or synchronous patterns with $\geq 50 \%$ suppression), or intermediate. Quantitative EEG analysis was performed every $2 \mathrm{~h}$ and included measures for amplitude, background continuity, burst-suppression amplitude ratio, and dominant frequency. Qualitative and quantitative measures were compared before and after the first propofol interruption. Additionally, the effect of propofol on each measure was estimated using linear mixed effects models, in which age, sex, time since cardiac arrest, resuscitation characteristics, and midazolam doses were included as covariates.

We included 496 patients, in whom the first propofol interruption took place at a median of $41 \mathrm{~h}$ after cardiac arrest. After interruption of propofol, the specificity of favorable EEG patterns for good outcome decreased, and the predictive value of unfavorable EEG patterns for poor outcome remained unchanged. Quantitatively, propofol was associated with decreases in EEG amplitude, background continuity and dominant frequency, and an increase of burst-suppression amplitude ratio. Despite these effects, propofol did neither increase the chance of an unfavorable EEG pattern (adjusted odds ratio (aOR) 0.92 per increase of 2 $\mathrm{mg} / \mathrm{kg} / \mathrm{h}, 95 \%-\mathrm{Cl}: 0.81-1.11)$ nor decrease the chance of a favorable EEG pattern (aOR 0.98 per increase of $2 \mathrm{mg} / \mathrm{kg} / \mathrm{h}, 95 \%-\mathrm{Cl}$ : 0.81-1.09).

We show that propofol changes the postanoxic EEG. However, its effects are too small to affect the reliability of EEG-based outcome predictions. 


\subsection{Introduction}

Postanoxic brain injury after cardiac arrest is among the most frequent causes of coma at the Intensive Care Unit (ICU). In approximately half of all patients, severe encephalopathy impedes recovery of consciousness. Patients are usually treated with targeted temperature management (TTM) at $32-36{ }^{\circ} \mathrm{C}$ and sedative medication for at least 24 hours. Although this therapy may improve neurological outcome, it interferes with most methods for prognostication, including the neurological examination ${ }^{1}$.

Electroencephalography (EEG) is a reliable tool for prediction of outcome within the first 24 hours after cardiac arrest, despite TTM and sedation ${ }^{2-4}$. Generalized suppression and synchronous patterns with $\geq 50 \%$ suppression (including generalized periodic discharges on a suppressed background and burst-suppression with generalized bursting and abrupt burst onsets, such as identical bursts) reliably predict a poor outcome. EEG patterns with a continuous background, in the absence of rhythmic or periodic activity, have a strong association with good recovery ${ }^{5}$. These predictors are equally reliable in centers using different sedation regimes ${ }^{6}$.

Sedative medication is well-known to change of the EEG. For example, propofol may induce burst-suppression patterns ${ }^{7}$, and midazolam may reduce the EEG amplitude ${ }^{8}$ and can also induce burst-suppression ${ }^{9}$. However, these changes of the EEG are typically induced at much higher doses than those used for sedation in the ICU ${ }^{10}$. Also, medication induced burst suppression has a different appearance than ischemia induced patterns ${ }^{11}$.

A recent explorative study investigated quantitative EEG ( $q E E G$ ) changes after sedation interruptions in relation to outcome ${ }^{12}$. Sedation altered EEG amplitude and continuity but did not importantly affect the relation between EEG parameters and neurological outcome. The sample size of this study was small, and the type of sedative medication and timing of the EEG varied widely.

In the current study, we focus on the effects of propofol, a commonly applied sedative drug on the ICU. We aim to quantify its effects on EEG patterns of comatose patients after cardiac arrest, and the consequent possible interference with outcome predictions. The analysis consists of two parts. In the first part, we quantify changes of the EEG shortly after interruption of propofol sedation and assess whether this information changes the predictive value of the EEG. In the second part, we estimate the effects of propofol on qualitative and quantitative EEG measures in multivariate models.

\subsection{Methods}

\subsubsection{Study design and participants}

This is a retrospective analysis of a prospective cohort study conducted in five centers in The Netherlands. We used data from three centers for which continuous data on intravenously administered medication were available (Medisch Spectrum Twente, St. Antonius Hospital, University Medical Center Groningen). Consecutive, comatose (Glasgow Coma Scale <8), adult patients, admitted to the ICU after cardiac arrest, were considered eligible for inclusion. In all three centers, continuous EEG monitoring is considered standard care for comatose patients after cardiac arrest. Part of the EEG 
data were previously used for studies on outcome prediction after cardiac arrest using visual $^{6}$ and quantitative analysis ${ }^{13}$. The Medical Research Ethics Committee Twente waived the need for informed consent for the EEG monitoring. Informed consent was obtained from surviving patients at time of follow up.

\subsubsection{Standard of care}

Patients were treated according to standard protocols for comatose patients after cardiac arrest. A target temperature of $33^{\circ} \mathrm{C}$ or $36^{\circ} \mathrm{C}$ was induced as soon as possible after arrival on the ICU and maintained for 24 hours. Patients were sedated with propofol, midazolam, or both, and received morphine, fentanyl, or remifentanil for analgesia. Propofol sedation was usually interrupted after the period of TTM, and restarted in case of severe arousals, prolonged cooling to suppress fever, myoclonus, or seizures.

\subsubsection{Decisions on withdrawal of life-sustaining treatment}

Withdrawal of treatment was considered $\geq 72$ hours after cardiac arrest, during normothermia and off sedation. Decisions on treatment withdrawal were based on international guidelines including bilateral absence of somatosensory evoked potentials (SSEP), absent or extensor motor responses, and absence of brainstem reflexes ${ }^{14,15}$. Decisions on treatment withdrawal were sporadically taken between 48 and 72 hours in case of absent SSEP responses. EEG data were not used for decisions regarding treatment withdrawal. However, physicians were not blinded to the EEG in order to allow for early detection and treatment of electrographic seizure activity.

\subsubsection{EEG recordings and analysis}

EEG recordings were started as soon as possible after admission to the ICU and, for practical reasons, only between 8 A.M. and 8 P.M. in each center, and not during weekend days in one center. Twenty-one cup electrodes were placed on the scalp according to the international 10-20 system. EEG recordings were continued until patients recoved consciousnes or died, with a maximum of five days.

All EEG analyses were performed offline, after the recordings. Visual analysis of EEG data was performed in a longitudinal bipolar montage, after band pass filtering ( 0.5 to $35 \mathrm{~Hz}$ ). Five-minute EEG epochs were assessed at 6, 12, 24, 36, 48, and $72 \mathrm{~h}$ after cardiac arrest, as described before ${ }^{6}$. EEG epochs were presented in random order to reviewers who were blinded to the timing of the epoch, the clinical condition of the patients, medication, and outcome. All EEG epochs were assessed by two experienced reviewers from a pool of six (B.R., M.T-C, M.v.P., H.K., A.G., or J.H.), independently. If the two reviewers disagreed, the final classification was determined by consensus. If necessary, a third reviewer was consulted. Reviewers were allowed to choose the option "no classification possible" if the epoch was considered unreliable due to artifacts.

On visual assessment, EEG patterns were classified as one of the following categories: generalized suppression (all activity $<10 \mu \mathrm{V}$ ), synchronous patterns with $\geq 50 \%$ suppression $(<10 \mu \mathrm{V})$, continuous, or intermediate. 'Synchronous patterns with $\geq 50 \%$ suppression' included generalized periodic discharges on a suppressed background and burst-suppression with generalized bursting and abrupt burst onsets. 'Continuous' included normal amplitude $(>20 \mu \mathrm{V})$ patterns with $<10 \%$ suppression, without peri- 
odic activity. 'Intermediate' patterns were subdivided in low voltage (maximum amplitude $10-20 \mu \mathrm{V}$ ), discontinuous (10-49\% suppression or attenuation), heterogeneous burst-suppression ( $\geq 50 \%$ suppression or attenuation, without generalized bursting or abrupt burst onsets), and epileptiform patterns other than GPDs on a suppressed background.

Quantitative analysis of the EEG was performed every two hours between 2 and 72 hours after cardiac arrest. Five-minute, artifact-free epochs were extracted automatically by a computer algorithm at the desired time $\pm 0.5 \mathrm{~h}$. The algorithm selected the five consecutive minutes closest to the reference time with artifact-parameters below a desired threshold, as described before ${ }^{5}$. No epoch was created if no artifact-free segment was found. For each EEG epoch, four qEEG features were extracted, after application of a 6-th order Butterworth band pass filter with range 0.5 to $30 \mathrm{~Hz}$ : power (POW), defined as the root mean squared amplitude, background continuity index $(\mathrm{BCl})$, defined as the fraction of EEG not spent in suppression (amplitude $<10 \mu \mathrm{V}$ for $\geq 0.5 \mathrm{~s}$ ), burst-suppression amplitude ratio (BSAR), defined as the mean amplitude ratio between non-suppressed and suppressed segments ${ }^{5}$, and alpha-delta ratio (ADR), defined as the power ratio between the alpha $(8-12 \mathrm{~Hz})$ and delta band $(1-4 \mathrm{~Hz})$. The BSAR was only calculated for $\mathrm{BCl}$ values between 0.05 and 0.95 . Power spectral density was calculated using Welch's averaged periodogram with a 50\% overlapping Hamming window of 10 s.

For the quantification of EEG changes at time of propofol interruption, two additional EEG epochs were extracted at $1 \mathrm{~h} \pm 0.5 \mathrm{~h}$ before and after the first propofol interruption.

\subsubsection{Additionally collected data}

For each patient, information on timing and dosage of continuously administered medication in the first five days after cardiac arrest was extracted semi-automatically from the patient data management system (Metavision). Additionally collected data include age, sex, and resuscitation details.

\subsubsection{Outcome}

The primary outcome measure was neurological functional recovery at six months, expressed as the score on the five-point Glasgow-Pittsburgh Cerebral Performance Category (CPC) (Jennett and Bond, 1975) dichotomized as good (CPC 1 or 2) or poor (CPC 3,4 , or 5). Outcome was assessed during a standardized telephone interview by one of two investigators (BR or MT-C) or a trained research nurse. CPC scores were based on a Dutch translation of the EuroQol-6D questionnaire. In one center, CPC scores were assessed using the Short Form 36 (SF-36) questionnaire. ${ }^{16}$

\subsubsection{Statistical analysis}

In order to compare baseline characteristics of patients with good and poor outcomes, categorical variables were analyzed using Pearson's $\chi^{2}$-test, and continuous variables using the Mann-Whitney test.

To compare the predictive values of the EEG during propofol sedation and after its interruption, we calculated test sensitivity and specificity (including 95\% confidence inter- 
vals) at $12 \mathrm{~h}$ after cardiac arrest, at the time of last assessment before the first propofol interruption, and the first assessment without propofol.

To compare qEEG features before and after propofol interruptions on a group level, we used Mann-Whitney tests. Aditionally, we investigated changes at the individual level. Since qEEG measures may to some extent fluctuate randomly over time, we tested each change for statistical significance. We defined a substantial increase as an increase greater than the 95th percentile value of changes between pairs of consecutive measurements not involved in a sedation transition. To compensate for ceiling and floor effects for the $\mathrm{BCl}$, which can only take values between 0 to 1 , we divided each change in the $\mathrm{BCl}$ by the maximum possible change. Similarly, to account for floor effects of the BSAR (with minimum value 1 ) we expressed decreases as the maximum possible decrease.

For analysis of the relation between propofol dose and the chance of a favorable (continuous) or unfavorable (suppression or synchronous pattern with $\geq 50 \%$ suppresssion) EEG pattern, we used mixed-effects logistic regression. In the regression models, propofol dose at time of extraction of the EEG epoch plus the following covariates were included as fixed terms: time after cardiac arrest, age, sex, location of cardiac arrest (in-hospital or out-of hospital), initial cardiac rhythm (ventricular fibrillation (VF) or other), cause of cardiac arrest (cardiac or noncardiac), time after cardiac arrest, midazolam dose at time of extraction of the EEG epoch, and an interaction term for midazolam and propofol doses. We included constant random effect terms for "patient" and "center".

For quantification of the effect of propofol on qEEG measures, we used generalized linear mixed-effects regression with the same set of covariates as used in the logistic regression analysis. In this analysis, we assumed a normal distribution of $\mathrm{BCl}$ and $\mathrm{BSAR}$ values, and gamma distributions for POW and ADR values. We used a logit link function to relate predictors to the $\mathrm{BCl}$, and a log link function to relate predictors to POW, BSAR, and ADR. For each model, we checked that residuals were normally distributed.

P-values $<0.05$ were considered statistically significant. All tests were performed using Matlab Statistics Toolbox software (MATLAB and Statistics Toolbox Release R2017b, The MathWorks, Inc., Natick, Massachusetts, United States).

\subsection{Results}

Between May 2010 and November 2017, EEG recordings were started in 506 patients. Ten had no artifact-free EEG at any of the investigated time points, leaving 496 patients for the analysis. Twenty patients without available outcome data were only used for part of the analysis.

\subsubsection{Clinical characteristics}

Clinical characteristics are shown in Table 5.1, grouped by outcome. Poor outcome occurred in 269 patients (57\%). Most differences were as expected: patients with poor outcome were older, more often had a non-cardiac cause of arrest, and less often had ventricular fibrillation (VF) as initial rhythm. Of note, patients with a good outcome required higher doses of propofol, fentanyl, and remifentanil, for sedation. 
Table 5.1: Patient characteristics, grouped by outcome.

\begin{tabular}{lccc}
\hline & $\begin{array}{c}\text { Poor outcome } \\
\text { (CPC 3-5) }\end{array}$ & $\begin{array}{c}\text { Good outcome } \\
(\text { CPC 1-2) }\end{array}$ & P-value \\
\hline Number & $269(57 \%)$ & $207(43 \%)$ & \\
Age & $67(55-74)$ & $59(50-69)$ & $<0.001$ \\
Female & $74(28 \%)$ & $49(24 \%)$ & 0.34 \\
Out-of-hospital cardiac arrest & $235(87 \%)$ & $192(93 \%)$ & 0.05 \\
Noncardiac cause of arrest & $60(26 \%)$ & $12(6 \%)$ & $<0.001$ \\
VF as initial cardiac rhythm & $135(55 \%)$ & $177(88 \%)$ & $<0.001$ \\
Hypothermia $\left(33^{\circ} \mathrm{C}\right)$ & $169(63 \%)$ & $138(67 \%)$ & 0.37 \\
EEG start time $(\mathrm{h}$ after cardiac arrest) & $10(5-18)$ & $9(5-16)$ & 0.177 \\
EEG stop time $(\mathrm{h}$ after cardiac arrest) & $56(41-96)$ & $52(43-76)$ & 0.686 \\
& & & \\
Treatment with propofol & $262(98 \%)$ & $204(99 \%)$ & 0.29 \\
$\quad$ Max. dose in first 24h $(\mathrm{mg} / \mathrm{kg} / \mathrm{h})$ & $3.0(2.3-3.7)$ & $3.5(2.7-4.1)$ & $<0.001$ \\
Treatment with midazolam & $53(20 \%)$ & $25(12 \%)$ & 0.03 \\
$\quad$ Max. dose in first $24 \mathrm{~h}(\mu \mathrm{g} / \mathrm{kg} / \mathrm{h})$ & $63(46-95)$ & $68(47-87)$ & 0.657 \\
Treatment with fentanyl & $160(60 \%)$ & $123(60 \%)$ & 1.00 \\
$\quad$ Max. dose in first $24 \mathrm{~h}(\mu \mathrm{g} / \mathrm{kg} / \mathrm{h})$ & $1.4(1.1-2.1)$ & $1.7(1.3-2.4)$ & 0.03 \\
Treatment with remifentanil & $33(12 \%)$ & $20(10 \%)$ & 0.37 \\
$\quad$ Max. dose in first $24 \mathrm{~h}(\mu \mathrm{g} / \mathrm{kg} / \mathrm{h})$ & $3.6(2.5-5.6)$ & $6.6(3.4-11.4)$ & 0.01 \\
Treatment with morphine & $54(20 \%)$ & $51(25 \%)$ & 0.23 \\
$\quad$ Max. dose in first $24 \mathrm{~h}(\mu \mathrm{g} / \mathrm{kg} / \mathrm{h})$ & $24(17-29)$ & $25(21-29)$ & 0.289 \\
SSEP performed & $177(66 \%)$ & $34(16 \%)$ & $<0.001$ \\
$\quad$ N20 bilaterally absent & $74(28 \%)$ & $0(0 \%)$ & $<0.001$ \\
& & & $<0.001$ \\
Propofol interruption $>1 \mathrm{~h}$ within $72 \mathrm{~h}$ & $170(63 \%)$ & $166(80 \%)$ & 0.09 \\
$\quad$ Time to interruption $(\mathrm{h})$ & $40(30-49)$ & $41(33-49)$ & 0.70 \\
$\quad$ Restart after interruption & $110(41 \%)$ & $81(39 \%)$ & \\
\hline
\end{tabular}

Data are shown as number (percentage) or median (interquartile range). SSEP: somatosensory evoked potential. P-value indicates differences between patients with poor and good outcome. VF: ventricular fibrillation. 
Patients with good outcome more often had an interruption of propofol sedation $\geq 1 \mathrm{~h}$ within the first $72 \mathrm{~h}$ than those with a poor outcome. In those with an interruption, the time to interruption and fraction in which propofol was restarted was not different between the outcome groups. For the quantification of EEG changes after propofol interruption, we excluded 20 patients with poor outcome (7\%) and 26 patients with a good outcome (13\%) due to artifacts.

All patients with generalized suppression or synchronous patterns with $\geq 50 \%$ suppression at $12 \mathrm{~h}$ had a poor outcome (CPC 3-5). In the remainder of the results, we will label these patterns as 'unfavorable'. Of those with a continuous pattern at $12 \mathrm{~h}, 92 \% \mathrm{had}$ a good outcome (CPC 1-2). In the remainder of the results, we will label these patterns as 'favorable'.

\subsubsection{Visual changes of the EEG after interruption of propofol}

Among patients with EEG started within $12 \mathrm{~h}$ after cardiac arrest, the EEG after interruption of propofol did not identify any new patients with poor outcome. Sensitivity for poor outcome was 0.18 (95\%-Cl: $0.12-0.26)$ after propofol interruption, as compared to $0.41(95 \%-\mathrm{Cl}: 0.32-0.49)$ at $12 \mathrm{~h}$, at equal reliability.

Propofol interruptions impaired the reliability of predictions of good outcome. The number of patients in whom at least one favorable EEG was observed increased after interruption of propofol, both in the good (96\% vs. $91 \%$ ) and poor outcome $(26 \%$ to $19 \%)$ group. The resulting increase of sensitivity for good outcome was not statistically significant $(0.81,95 \%-\mathrm{Cl}: 0.73-0.87$ vs. $0.78,0.70-0.84)$, but the loss of specificity was (0.63, $95 \%-\mathrm{Cl}: 0.54-0.70$ vs. $0.76,0.70-0.83)$. As compared to the EEG at $12 \mathrm{~h}$ after cardiac arrest,sensitivity for good outcome was higher after propofol interruptions (0.81, $95 \%-\mathrm{Cl}: 0.73-0.87$ vs. $0.42,95 \%-\mathrm{Cl}: 0.34-0.51)$, but specificity was much lower (0.63, 95\%-Cl: $0.54-0.70$ vs. $0.92,95 \%-C l: 0.86-0.96)$.

\subsubsection{Quantitative changes of the EEG after propofol interruptions}

Substantial changes in amplitude and background continuity after propofol interruption were associated with a good outcome (Figure 5.1). The median power of the EEG increased in patients with good outcome (POW: 10.7 vs. $9.0 \mu \mathrm{V}, \mathrm{p}<0.001$ ) but not in patients with poor outcome ( $8.5 \mathrm{vs} .5 .6 \mu \mathrm{V}, \mathrm{p}=0.07)$. Of note, patients with a good outcome more often had a substantial decrease of power, too ( $10 \%$ vs. $3 \%, p=0.02)$. Both a substantial increase (OR: $2.46,95 \%-\mathrm{Cl}: 1.36-4.44)$ and a substantial decrease in power (OR 6.15, 95\%-Cl: 1.70-22.2) after propofol interruption were associated with good recovery.

Background continuity was higher after propofol interruptions, both in patients with good outcome $(\mathrm{BCl}: 1.00$ vs. $0.98, \mathrm{p}<0.001)$ and in those with poor outcome (median $\mathrm{BCl}$ : 0.93 vs. $0.75, \mathrm{p}=0.04)$. Again, the fraction of patients with a substantial increase was higher in the good outcome group ( $50 \%$ vs. 33\%, $p=0.01$ ).

The median alpha-delta ratio did not change after propofol interruption in patients with good outcome (ADR: 0.12 vs. $0.08, p=0.09$ ) or poor outcome (0.09 vs. $0.09, p=0.59$ ). There was no difference in the fraction of patients with a substantial increase in ADR between the outcome groups ( $16 \%$ vs. $10 \%, p=0.14)$. Likewise, the amplitude ratio 

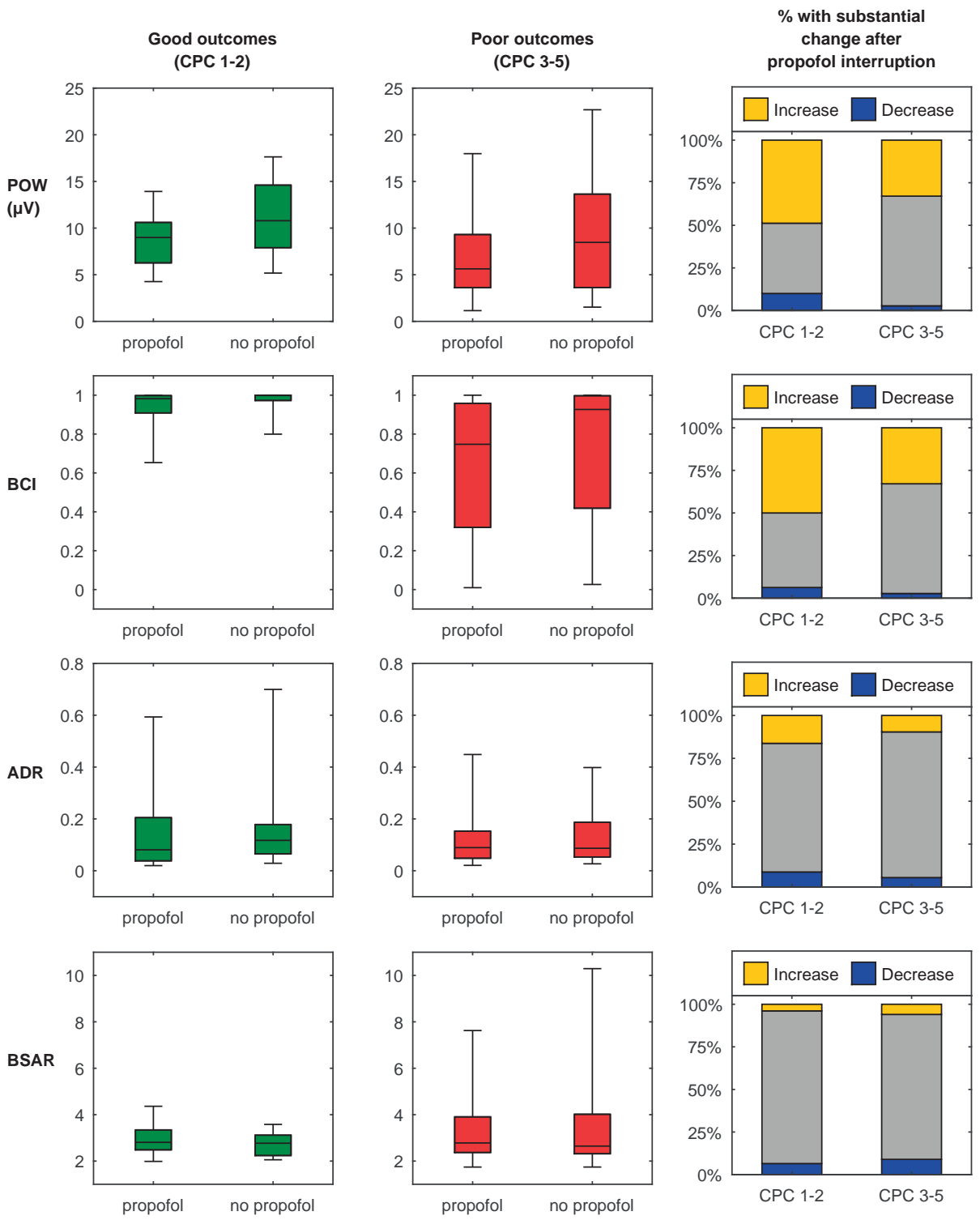

Figure 5.1: Quantitative EEG changes after interruption of propofol sedation. Each row shows changes for one of the qEEG features. The first two columns compare the distribution of qEEG values, grouped by outcome, at $1 \pm 0.5 \mathrm{~h}$ before the first propofol interruption ('propofol'), and $1 \pm 0.5 \mathrm{~h}$ after the first propofol interruption ('no propofol'). Horizontal lines indicate medians, boxes interquartile ranges, and error bars range from the 5 th to 95 th percentile values. The last column shows the fraction of patients with a substantial increase ( $>95$ th percentile of reference values) or decrease ( $<5$ th percentile of reference values). The fraction of patients without substantial change is shown in grey. POW: power, $\mathrm{BCl}$ : background continuity index, ADR: alpha-delta ratio, BSAR: burst-suppression amplitude ratio. 


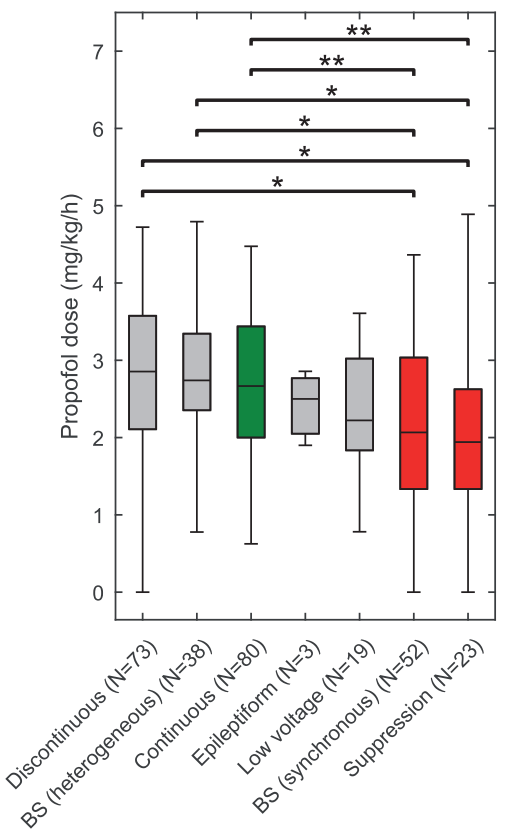

Figure 5.2: Distribution of propofol doses, given the EEG pattern at $12 \mathrm{~h}$ after cardiac arrest. EEG categories are sorted by the median propofol doses, in descending order. Green boxes indicate favorable categories, red boxes unfavorable categories, and grey boxes intermediate categories. In each boxplot, horizontal lines indicate medians, the box the interquartile range, and the error bars the 5th and 95th percentile values. In the comparison of propofol doses between categories, * indicates $p<0.05$, and ${ }^{* *}$ indicates $p<0.01$ for the difference, as determined by the Mann-Whitney test. BS: burst-suppression.

between bursts and suppressions (BSAR) did neither change in patients with good outcome (median BSAR: 2.77 vs. $2.81, \mathrm{p}=0.15$ ) nor in patients with poor outcome (2.65 vs. $2.78, p=0.56)$. The fraction of patients with a substantial increase or decrease was below $10 \%$ in both outcome groups.

\subsubsection{Relation between propofol dose and EEG category on visual assessment} In general, propofol dosages at $12 \mathrm{~h}$ after cardiac arrest were lower for patients with unfavorable EEG patterns as compared with patients with other patterns (Figure 5.2). The highest median doses were observed during discontinuous patterns, heterogeneous burst-suppression, and continuous patterns (2.85, 2.74, $2.67 \mathrm{mg} / \mathrm{kg} / \mathrm{h}$, respectively). The median propofol dose during these patterns was significantly higher than during synchronous burst-suppression ( $2.07 \mathrm{mg} / \mathrm{kg} / \mathrm{h}, \mathrm{p}=0.02, \mathrm{p}=0.02, \mathrm{p}=0.007$, respectively) and generalized suppression ( $1.94 \mathrm{mg} / \mathrm{kg} / \mathrm{h}, \mathrm{p}=0.01, \mathrm{p}=0.01, \mathrm{p}=0.01$, respectively).

In the mixed-effects logistic regression analysis, propofol did neither decrease the chance of a favorable EEG pattern (adjusted odds ratio (aOR): 0.98 per increase of 2 $\mathrm{mg} / \mathrm{kg} / \mathrm{h}, 95 \%-\mathrm{Cl}: 0.81-1.09)$ nor increase the chance of an unfavorable EEG pattern (aOR: 0. 95 per increase of $2 \mathrm{mg} / \mathrm{kg} / \mathrm{h}, 95 \%-\mathrm{Cl}$ : 0.81-1.11). Midazolam was also not 

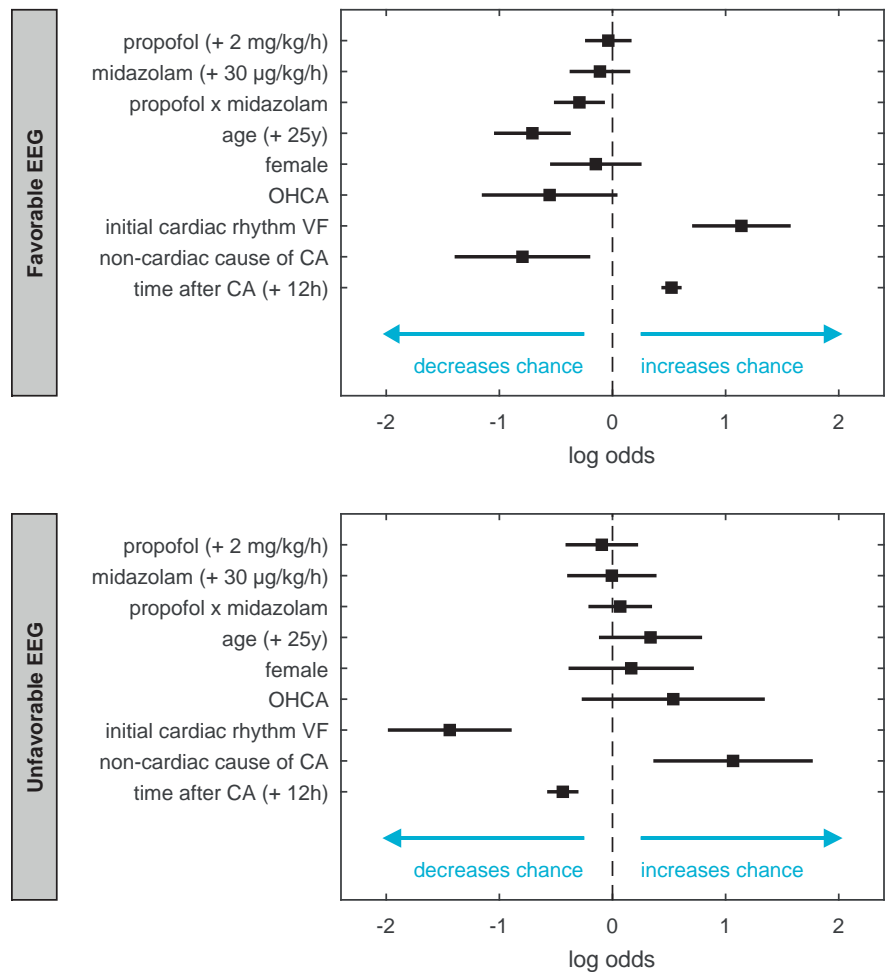

Figure 5.3: Effects of individual predictors in mixed-effects logistic regression on chance of favorable or unfavorable EEG pattern. Squares indicate beta coefficients of the predictors, horizontal lines the corresponding 95\% confidence intervals. CA: cardiac arrest, VF: ventricular fibrillation, OHCA: out of hospital cardiac arrest. Propofol $x$ midazolam indicates to the interaction effect between propofol and midazolam; the effect size refers to an increase in propofol dose with $2 \mathrm{mg} / \mathrm{kg} / \mathrm{h}$ plus an increase in midazolam dose with $30 \mu \mathrm{g} / \mathrm{kg} / \mathrm{h}$.

associated with the chance of a favorable or unfavorable EEG pattern. However, a combination of propofol and midazolam decreased the chance of a favorable EEG pattern (aOR (aOR: $7.7 \cdot 10^{3}, 95 \%-\mathrm{Cl}: 1.8 \cdot 10^{4}-0.33$ ) (Figure 5.3).

\subsubsection{Relation between propofol dose and quantitative EEG measures}

Higher propofol doses were associated with statistically significant changes of any of the qEEG measures (Figure 5.4). For the median propofol dose at $12 \mathrm{~h}$ after cardiac arrest $(2.66 \mathrm{mg} / \mathrm{kg} / \mathrm{h})$, the root mean squared power (POW) decreased with $23 \%$ (95\%$\mathrm{Cl}: 12-33 \%)$, the background continuity index (BCl) decreased with 16\% (95\%-Cl: 9$32 \%)$, the alpha delta ratio (ARD) decreased with 29\% (95\%-Cl: 16-39\%), and the burstsuppression amplitude ratio (BSAR) increased with $13 \%$ (95\%-Cl: 4-31\%). Note that these values, as estimated by linear mixed-effects regression, agree well with the order of magnitude of the actually observed changes in qEEG measures after the interruption of propofol (Figure 5.1). Results were qualitatively the same in both outcome groups, indicating lack of any association between propofol induced EEG changes and outcome. 

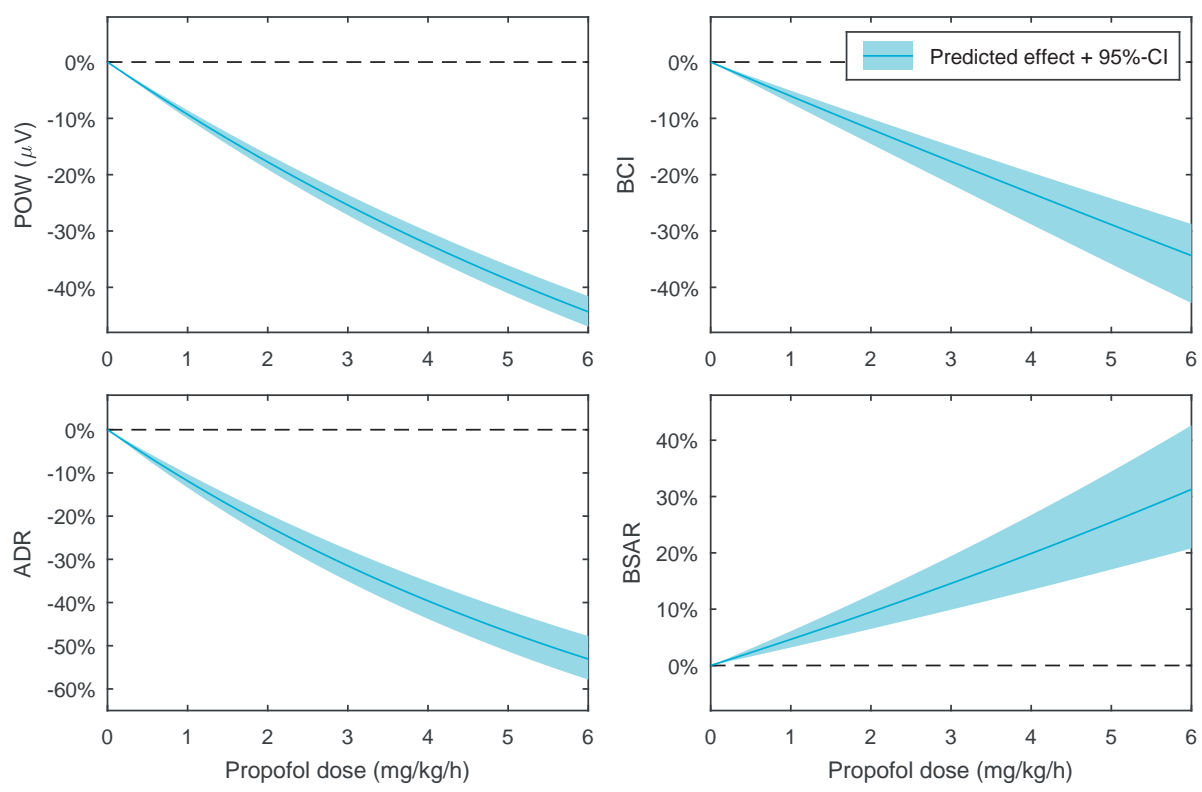

Figure 5.4: Change of quantitative EEG measures for increasing propofol doses, as estimated by linear mixedeffects regression. Blue solid lines represent the predicted change, purple areas the corresponding $95 \%$ confidence intervals. POW: power, $\mathrm{BCl}$ : background continuity index, BSAR: burst-suppression amplitude ratio, ADR: alpha-delta ratio

\subsection{Discussion}

We investigated the effects of propofol sedation on EEG patterns of comatose patients after cardiac arrest. We show that propofol does neither decrease the chance of visually adjudicated favorable EEG patterns, nor increase the chance of unfavorable patterns, despite small quantitative effects on amplitude, background continuity, dominant frequency, and burst-suppression amplitude ratio. The EEG after sedation propofol interruptions, which took place at a median of $41 \mathrm{~h}$ after cardiac arrest, did not add to prediction of poor outcome. Quantitatively, increases changes of the EEG after sedation interruption, including an increase in amplitude and background continuity after propofol interruption, were more pronounced in patients with a good outcome.

Several studies have shown that EEG-based predictors of poor and good outcome of comatose patients after cardiac arrest are robust, despite various treatment regimens with sedative medication ${ }^{2-4,6,17}$. However, since interpretation of the EEG critically depends on amplitude and background continuity, treatment with propofol or other sedative medication is often assumed to hamper the value of the EEG for outcome prediction. We now present direct evidence that propofol sedation does not affect the reliability of the EEG for prediction of poor outcome and may enhance predictions of good outcome. We could not demonstrate an association of midazolam sedation with a change in EEG-based outcome prediction, but numbers of patients treated with midazolam were small and confidence intervals were wide. When propofol and midazolam were given in combination, we observed a reduction of the change of a favorable EEG 
pattern, however without increasing the chance of an unfavorable EEG pattern.

The observed changes in the qEEG after propofol interruption confirm recent findings ${ }^{12}$, using a much larger sample with less heterogeneity in sedation regimes, better defined timing of EEG assessment, and a clear outcome measure. Our regression analysis allows for direction predictions of the effects of increasing propofol doses on qualitative and quantitative measures of EEG.

On a group level, propofol doses were lower in patients with unfavorable EEG patterns, as compared with other patients. Most likely, this follows from the fact that patients with severe brain injury have lower sedation requirements. Also, hemodynamic instability in severely affected patients may limit the maximum possible dose of propofol. To account for this effect, we included other predictors of the severity of brain injury as covariates when estimating the effects of propofol on the EEG. The agreement between predicted and observed effects in qEEG measures after interruption of propofol suggest that our analysis is accurate.

In the range from 0 to $6 \mathrm{mg} / \mathrm{kg} / \mathrm{h}$ propofol, the estimated decrease of background continuity was never more than $40 \%$. This suggests that propofol, in the typical doses used, will on its own not induce burst-suppression patterns. However, a combination of propofol and midazolam was associated with a reduction of the chance of a continuous EEG pattern. This is most likely the result of their additional effects on the EEG, but may partly be explained by the fact that epileptiform activity was the reason to use this combination in part of the patients.

This study has limitations. We cannot exclude that a self-fulfilling prophecy has biased our results related to the outcome of patients ${ }^{19}$. To minimize this risk, decisions on treatment withdrawal were based on international guidelines including bilaterally absent SSEP, absent or extensor motor responses, and absent brain stem reflexes ${ }^{14,15}$. EEG patterns observed in the first $72 \mathrm{~h}$ after cardiac arrest were not taken into account. In the regression analyses, where we related propofol doses to visual and quantitative EEG characteristics, we used medication doses during the recording of the epoch. Ongoing effects of medication administered before that time may have blurred our results to some extent. The fact that propofol interruptions for more than 1 hour were less often possible in patients with poor outcome may have biased our results on EEG changes after the interruption of propofol.

\section{References}

1. Sandroni C, Cavallaro F, Callaway CW, D'Arrigo S, Sanna T, Kuiper Ma, et al. Predictors of poor neurological outcome in adult comatose survivors of cardiac arrest: a systematic review and meta-analysis. Part 2: Patients treated with therapeutic hypothermia. Resuscitation 2013; 84: 1324-1338.

2. Sivaraju A, Gilmore EJ, Wira CR, Stevens A, Rampal N, Moeller JJ, et al. Prognostication of post-cardiac arrest coma: early clinical and electroencephalographic predictors of outcome. Intensive Care Medicine 2015; 41: 1264-1272.

3. Sondag L, Ruijter BJ, Tjepkema-Cloostermans MC, Beishuizen A, Bosch FH, van Til JA, et al. Early EEG for outcome prediction of postanoxic coma: prospective cohort study with costminimization analysis. Critical Care 2017; 21: 111. 
4. Spalletti M, Carrai R, Scarpino M, Cossu C, Ammannati A, Ciapetti M, et al. Single electroencephalographic patterns as specific and time-dependent indicators of good and poor outcome after cardiac arrest. Clinical Neurophysiology 2016; 127: 2610-2617.

5. Ruijter BJ, Hofmeijer J, Tjepkema-Cloostermans MC, van Putten MJAM. The prognostic value of discontinuous EEG patterns in postanoxic coma. Clinical Neurophysiology 2018; 129: 1534-1543.

6. Ruijter BJ, Tjepkema-Cloostermans MC, Tromp SC, Van den Bergh WM, Foudraine NA, Kornips $\mathrm{FH}$, et al. Early EEG for outcome prediction of postanoxic coma: a prospective multicenter cohort study. Submitted 2018; .

7. San-Juan D, Chiappa KH, Cole AJ. Propofol and the electroencephalogram. Clinical Neurophysiology 2010; 121: 998-1006.

8. Veselis RA, Reinsel R, Marino P, Sommer S, Carlon GC. The effects of midazolam on the EEG during sedation of critically ill patients. Anaesthesia 1993; 48: 463-470.

9. ter Horst $\mathrm{H}$, Brouwer $\mathrm{O}$, Bos A. Burst suppression on amplitude-integrated electroencephalogram may be induced by midazolam: a report on three cases. Acta Paediatrica 2004; 93 : 559-563.

10. Huotari AM, Koskinen M, Suominen K, Alahuhta S, Remes R, Hartikainen KM, et al. Evoked EEG patterns during burst suppression with propofol. British Journal of Anaesthesia 2004; 92: $18-24$.

11. Hofmeijer J, Tjepkema-Cloostermans MC, van Putten MJAM. Burst-suppression with identical bursts: A distinct EEG pattern with poor outcome in postanoxic coma. Clinical Neurophysiology 2014; 125: 947-954.

12. Drohan CM, Cardi AI, Rittenberger JC, Popescu A, Callaway CW, Baldwin ME, et al. Effect of sedation on quantitative electroencephalography after cardiac arrest. Resuscitation 2018; 124: $132-137$.

13. Tjepkema-Cloostermans MC, Hofmeijer J, Beishuizen A, Hom HW, Blans MJ, Bosch FH, et al. Cerebral Recovery Index: Reliable help for prediction of neurologic outcome after cardiac arrest. Critical Care Medicine 2017; 45: 789-797.

14. Sandroni C, Cariou A, Cavallaro F, Cronberg T, Friberg H, Hoedemaekers C, et al. Prognostication in comatose survivors of cardiac arrest: An advisory statement from the European Resuscitation Council and the European Society of Intensive Care Medicine. Resuscitation 2014; 85: 1779-1789.

15. Wijdicks EFM, Hijdra A, Young GB, Bassetti CL, Wiebe S. Practice parameter: prediction of outcome in comatose survivors after cardiopulmonary resuscitation (an evidence-based review): report of the Quality Standards Subcommittee of the American Academy of Neurology. Neurology 2006; 67: 203-210.

16. Ruijter BJ, van Putten MJ, Horn J, Blans MJ, Beishuizen A, van Rootselaar AF, et al. Treatment of electroencephalographic status epilepticus after cardiopulmonary resuscitation (TELSTAR): study protocol for a randomized controlled trial. Trials 2014; 15: 433.

17. Hofmeijer J, Beernink TMJ, Bosch FH, Beishuizen A, Tjepkema-Cloostermans MC, Van Putten MJAM. Early EEG contributes to multimodal outcome prediction of postanoxic coma. Neurology 2015; 85: 137-143.

18. Hofmeijer J, van Putten MJAM. Ischemic cerebral damage: an appraisal of synaptic failure. Stroke 2012; 43: 607-615.

19. Geocadin RG, Peberdy MA, Lazar RM. Poor survival after cardiac arrest resuscitation. Critical Care Medicine 2012; 40: 979-980. 


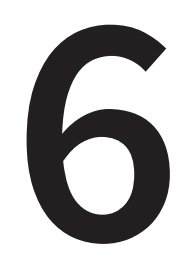

\section{Synaptic damage underlies EEG abnormalities in postanoxic encephalopathy: a computational study}

Published as: B.J. Ruijter, J. Hofmeijer, H.G.E. Meijer, M.J.A.M. van Putten, Synaptic damage underlies EEG abnormalities in postanoxic encephalopathy: a computational study, Clinical Neurophysiology 2017; 128: 16821695. 


\section{Abstract}

Objective

Methods

Results

Conclusions

Significance
In postanoxic coma, EEG patterns indicate the severity of encephalopathy and typically evolve in time. We aim to improve the understanding of pathophysiological mechanisms underlying these EEG abnormalities.

We used a mean field model comprising excitatory and inhibitory neurons, local synaptic connections, and input from thalamic afferents. Anoxic damage is modeled as aggravated short-term synaptic depression, with gradual recovery over many hours. Additionally, excitatory neurotransmission is potentiated, scaling with the severity of anoxic encephalopathy. Simulations were compared with continuous EEG recordings of 155 comatose patients after cardiac arrest.

The simulations agree well with six common categories of EEG rhythms in postanoxic encephalopathy, including typical transitions in time. Plausible results were only obtained if excitatory synapses were more severely affected by short-term synaptic depression than inhibitory synapses.

In postanoxic encephalopathy, the evolution of EEG patterns presumably results from gradual improvement of complete synaptic failure, where excitatory synapses are more severely affected than inhibitory synapses. The range of EEG patterns depends on the excitation-inhibition imbalance, probably resulting from long-term potentiation of excitatory neurotransmission.

Our study is the first to relate microscopic synaptic dynamics in anoxic brain injury to both typical EEG observations and their evolution in time. 


\subsection{Introduction}

Of all comatose survivors of cardiac arrest, $46-48 \%$ are alive and independent in activities of daily living after 6 months ${ }^{1,2}$. Continuous electroencephalography (cEEG) in the first 24 hours after resuscitation allows reliable identification of $50 \%$ of patients with either a good or poor outcome ${ }^{1}$. Not only the EEG abnormalities as such, but also their timing and evolution are crucial indicators of the severity of the ischemic injury and prognosis ${ }^{3}$. In the first days after an anoxic event, the EEG usually evolves in fixed sequences ${ }^{3-6}$, and some EEG patterns are highly specific for postanoxic encephalopathy ${ }^{7}$. Some of the transitions observed indicate good neurological recovery, whereas other have a strong association with a poor outcome. The common evolutional pathway for patients with a good outcome is from no measurable cortical activity ("isoelectric" EEG), via intermittent cortical activity ("burst-suppression EEG") to continuous activity $^{5}$. For patients with poor outcome, either a delayed evolution ${ }^{1}$, or highly specific EEG patterns are observed. Examples of the latter are "burst-suppression with identical bursts" ${ }^{7}$ and generalized periodic discharges (GPDs) on an isoelectric background ${ }^{8}$. While these various EEG patterns and transitions can be related to patients' outcome, the pathophysiologiccal mechanisms underlying these EEG abnormalities remain unclear.

EEG activity is mainly a reflection of cortical synaptic activity ${ }^{9}$. Therefore, EEG patterns observed in postanoxic encephalopathy reflect changes at the synaptic level, especially because synaptic failure is an early event observed in case of cerebral hypoxia ${ }^{10}$. A better understanding of synaptic mechanisms underlying the EEG in postanoxic encephalopathy will contribute to knowledge on the pathophysiology, and possibly open opportunities for treatment.

Neural mean field models can be used to relate microscopic properties of neurons to macroscopic network behavior, reflected by EEG rhythms ${ }^{11,12}$. In such models, individual cell properties and their interactions are replaced by continuous functions that depend on some form of spatial averaging. Neural mean field models have been successfully used, for example, to simulate effects of anesthetics on the EEG ${ }^{13}$, epileptic seizures ${ }^{14}$, intermittent spike-wave dynamics ${ }^{15}$, and high-frequency oscillations ${ }^{16}$. With respect to postanoxic encephalopathy, a neural mean field model revealed a possible mechanisms underlying GPDs ${ }^{17}$. However, other pathological EEG patterns, like burst suppression with identical bursts ${ }^{7}$, and pattern transitions as observed in postanoxic encephalopathy remain unexplained.

A relevant mechanism of synaptic failure in postanoxic encephalopathy is short-term synaptic depression, also called activity-dependent synaptic depression ${ }^{18}$ : synapses need time to recover after signal transmission, for example to restore ion and neurotransmitter gradients. High neural firing rates limit the maximally achievable postsynaptic currents. This mechanism plays a role in physiological situations, and is probably aggravated in postanoxic encephalopathy. Specifically, this may be the result of presynaptic mechnisms, such as a dysfunction of plasma membrane $\mathrm{Ca}^{2+}$ extrusion systems comprised of $\mathrm{Ca}^{2+}$ pumps and $\mathrm{Na}^{+} / \mathrm{Ca}^{2+}$ exchangers ${ }^{19}$ and a disturbed undocking of synaptic vesicles from the reserve pool attributable to impaired phosphorylation ${ }^{20}$. We assume that these effects are potentially reversible on a time scale of 24 
to 72 hours, in line with the typical recovery time of the EEG in postanoxic encephalopathy ${ }^{3-6}$.

A second mechanism, taking place in severe postanoxic encephalopathy, is potentiation of excitatory neurotransmission. This mechanism has been substantiated by experimental observations in hippocampal slices exposed to anoxia ${ }^{21,22}$, and is compatible with the apparent network hyperexcitability in patients with severe postanoxic encephalopathy, reflected by a frequent observation of epileptiform discharges ${ }^{8,23,24}$. The most likely cause is anoxic long-term potentiation (LTP) of excitatory cortical synapses following anoxic depolarization ${ }^{25-27}$. This effect is caused by an increase of extracellular glutamate concentrations, resulting from reversed uptake of glutamate in neurons and glia cells ${ }^{28}$. In combination with anoxic depolarization, this induces a long-term potentiaton of N-methyl-D-aspartate (NMDA)-receptor gated currents ${ }^{27}$.

In this study, we aim to identify candidate synaptic mechanisms underlying typical EEG patterns and transitions in postanoxic encephalopathy by means of a neural mean field model. We hypothesize that, if synaptic recovery occurs in postanoxic encephalopathy, this is driven by a gradual improvement of short-term synaptic depression on a time scale of 24 to 72 hours, and reflected by improvement of the EEG. Furthermore, we hypothesize that increasing severity of hypoxia leads to a stronger potentiation of excitatory synaptic transmission. We incorporated both these key mechanisms into our computational model. The simulated EEG patterns will be related to clinical EEG data of patients with postanoxic encephalopathy after cardiac arrest.

\subsection{Methods}

\subsubsection{Clinical data collection}

Continuous EEG recordings were collected between June 2010 and December 2015 in a large teaching hospital in The Netherlands (Medisch Spectrum Twente, Enschede). All patients who were admitted comatose after a cardiac arrest were included, if it was possible to start EEG recordings within 12 hours after resuscitation. Two-thirds of the selected patients were also included in previous studies on outcome prediction after cardiac arrest ${ }^{1,3,4}$. Twenty-one silver/silver chloride cup electrodes were placed on the scalp according to the international 10-20 system. EEG recordings were continued until patients were awake or until the decision to withdraw treatment was made, with a maximum of five days. Additionally collected clinical data include age, gender, resuscitation details, and maximum levels of sedative medication. EEG data were not used for decisions regarding treatment withdrawal. However, treating physicians were not blinded to the EEG and treatment of electrographic seizures was left to the discretion of the treating physician. The primary outcome measure was the Cerebral Performance Category (CPC) at six months after cardiac arrest, assessed by a telephone interview. These scores were dichotomized into "good" (CPC 1-2, i.e. no or moderate neurological disability) and "poor" (CPC 3-5, i.e. severe disability, coma, or death). The Medical Ethics Committee Twente approved the protocol and waived the need for informed consent for EEG monitoring during the ICU stay and clinical follow-up. 


\subsubsection{Statistical analysis}

In order to compare patients with good and poor outcomes, continuous variables were compared using independent samples t-tests and binary variables using $\chi^{2}$-tests. Pvalues $<0.05$ were considered statistically significant.

\subsubsection{Basic computational model}

We used an adapted, spatially homogeneous version of the "bursting Liley model" 13,29, ${ }^{30}$ for our simulations. This model was used previously to simulate burst-suppression patterns ${ }^{29}$. Figure 6.1A gives an overview of the model. It consists of one population of excitatory (pyramidal) neurons and one population of inhibitory (inter-)neurons. Excitatory neurons have synaptic projections to inhibitory neurons and to themselves. Similarly, inhibitory neurons have projections to excitatory neurons and to themselves. The spike rates of the neural populations depend on the mean membrane potential. In a hyperpolarized state, neurons will stop firing, and above a certain threshold potential firing rates will saturate to a maximum frequency (Figure 6.1B). All connections between neurons are synaptic connections. Postsynaptic membrane potentials are given by the convolution of the incoming firing rate with the "synaptic response function", as shown in Figures 6.1C and 6.1D. The peak amplitudes of the synaptic response functions are referred to as $\Gamma_{e}$ and $\Gamma_{i}$ for excitatory and inhibitory synapses, respectively.

The bursting Liley model includes short-term synaptic depression. This implies that the postsynaptic peak amplitudes $\Gamma_{e}$ and $\Gamma_{i}$ decrease as a function of presynaptic firing rates and recover with time constants $\tau_{e}^{r e c}$ and $\tau_{i}^{r e c}$, respectively. Without noisy input from thalamic fibers, the average membrane potentials remain at some equilibrium value. For presynaptic firing rates above this equilibrium value postsynaptic peak amplitudes will decrease, and for presynaptic firing rates below the equilibrium value postsynaptic peak amplitudes will increase (see Appendix A for model details).

\subsubsection{Adaptations to the Bursting Liley model}

To model the evolution of the EEG, we assumed the short-term synaptic recovery time constants $\tau_{e}^{r e c}$ and $\tau_{i}^{r e c}$ to vary slowly, on a time scale of hours. We hypothesize that, briefly after the anoxic event, these constants are very high ( $\geq 100$ times baseline value) and then slowly decay to their baseline values. This behaviour reflects the slow recovery of presynaptic metabolic processes in the postanoxic period. To model the effect of anoxic long-term potentiation of excitatory synapses, we increased the maximum amplitude of EPSPs $\left(\Gamma_{e}\right)$. The effect is shown in Figure 6.1D. Candidate mechanisms include an increased expression of NMDA receptors or long-lasting elevations of modulators that potentiate NMDA-receptor opening, induced by the brief anoxic period ${ }^{27}$. An alternative scenario, which we will not consider here, would be to increase the EPSP decay time, resulting in similar overall behaviour. In the following, we will indicate the fractional increase in $\Gamma_{e}$ as the "LTP-factor", with a higher LTP factor indicating more severe hypoxic damage. Note, that in the original bursting Liley model $\Gamma_{e}$ is reduced and may recover with time constant $\tau_{e}^{r e c}$ to its baseline value, while in our adaptation of the Liley model $\Gamma_{e}$ may increase above its baseline value, reflecting excitatory potentiation. See Appendix A for details on the model equations. 
A
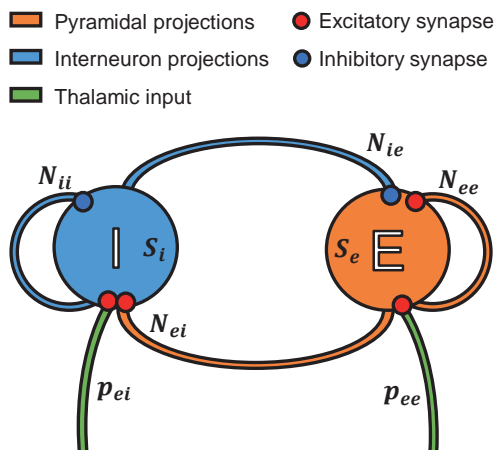

C

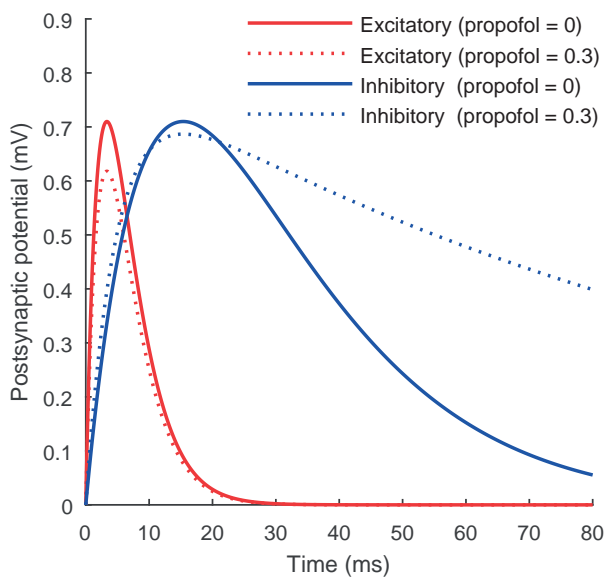

B

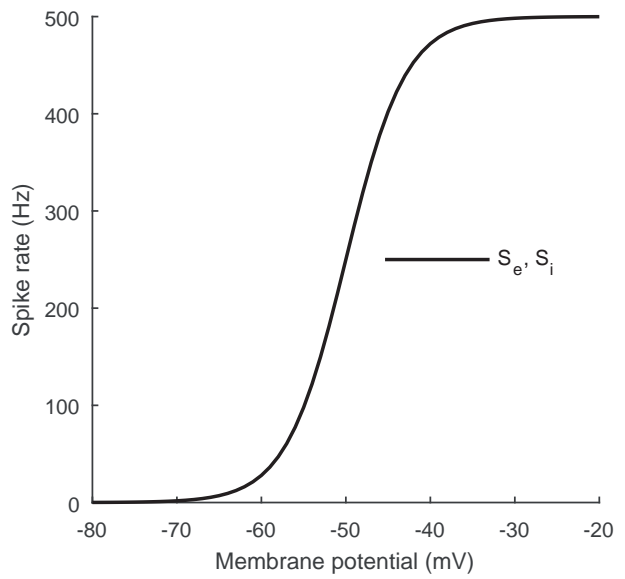

D

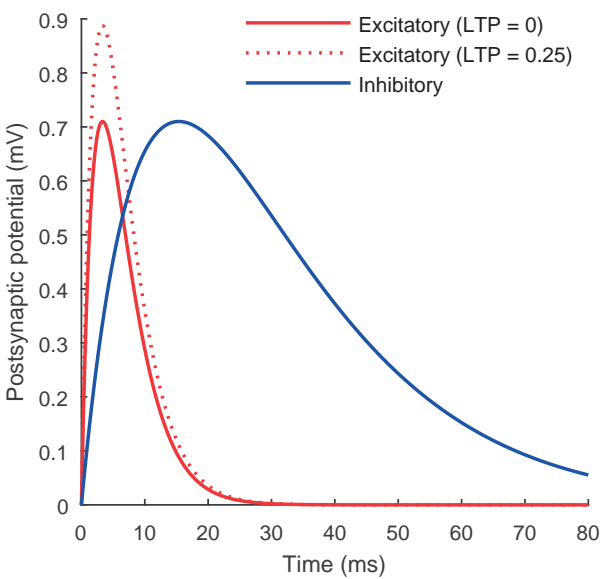

Figure 6.1: Overview of computational model, including activation function and synaptic responses. A: Sketch of the spatially homogeneous Bursting Liley model. It comprises one excitatory population (E) and one inhibitory neural population (I). Excitatory synapses are indicated with red disks, inhibitory synapses with blue disks. The green lines indicate input from thalamic fibers. The symbols indicate the various inputs to the excitatory and inhibitor population, respectively. Details on these parameters can be found in Table 6.A.1. B: The "activation function" for both the excitatory (E) and inhibitory (I) population, showing the relationship between mean membrane potential and population spiking rates. C: Synaptic response functions for excitatory (red) and inhibitory (blue) synapses. Curves represent the induced postsynaptic potential for a unit of presynaptic input. The continuous lines indicate the baseline response, the dashed lines the responses after application of a high dose of propofol. Note that we used a higher dose here $(0.25$ units) as compared to the simulations (0.15), in order to illustrate the effect. The peak values of the postsynaptic potential are referred to as $\Gamma_{e}$ or $\Gamma_{i}$, respectively. D: Same synaptic response functions as in C, but with the dashed line indicating the excitatory response in case of long-term potentiation (LTP). 


\subsubsection{Effects of anesthetic drugs}

Most patients admitted to the Intensive Care Unit after a cardiac arrest are treated with targeted temperature management and sedation ${ }^{2}$. In Medisch Spectrum Twente, patients are usually sedated with propofol. The bursting Liley model includes the effect of isoflurane, another general sedative agent. Figure 6.1C shows how sedation modifies the synaptic response function. Sedation increases the duration of inhibitory postsynaptic currents (IPSPs) and reduces the peak amplitude of excitatory postsynaptic currents (EPSPS). Although the model is quantitatively based on the effects of isoflurane, we assume propofol to have the same qualitative effects, since the $\mathrm{GABA}_{A}$ receptor is the most important target site of both drugs ${ }^{31}$.

\subsubsection{Numerical simulations}

Differential equations were solved using the Euler-Maruyama method with a time step of $0.1 \mathrm{~ms}$. For all simulations, we used the parameter values listed in Table 6.A.1, unless stated otherwise. The basic set of parameters was chosen to generate a physiological alpha-rhythm ${ }^{17,30}$. In all simulations, EEG signals of 75 seconds were generated. The first 15 seconds of each simulation were ignored to exclude transient effects. The membrane potential $V_{e}(t)$ of the excitatory population was used to represent the EEG signal, as was done for example in ${ }^{29}$. The simulations thus resulted in a single time series, reflecting a single channel representation of the EEG. We considered this to be sufficient, since EEG abnormalities in postanoxic encephalopathy are typicially spatially homogeneous.

Three parameters were varied in the simulations. Both the excitatory $\left(\tau_{e}^{r e c}\right)$ and inhibitory $\left(\tau_{i}^{r e c}\right)$ synaptic recovery time constants were varied between their baseline value $(500 \mathrm{~ms})$ and $10^{7} \mathrm{~ms}$. The chosen baseline value agrees with the physiological range of $250 \mathrm{~ms}$ to $1000 \mathrm{~ms}^{18}$. Because excitatory synapses probably recover slower than inhibitory synapses, we only considered simulations with $\tau_{e}^{r e c} \geq \tau_{i}^{r e c}$ as physiologically plausible ${ }^{17,32}$. The LTP-factor was varied between 0 and 5 . The order of magnitude for the LTP-factor was chosen based on experimental work in hippocampal slices after anoxia, where the maximum EPSP slope increased already twofold after 5 minutes of anoxia ${ }^{22}$. Simulations were performed both without the effect of propofol and with a moderate dose of propofol (0.15 units), using Matlab (MATLAB and Statistics Toolbox Release R2015b, The MathWorks, Inc., Natick, Massachusetts, United States).

\subsubsection{Categorization of clinical and simulated EEG data}

EEG analysis was performed offline. Before any analysis, clinical EEG data were transformed to the longitudinal bipolar montage. Five minute artifact-free epochs were selected automatically every hour, as described previously in ${ }^{33}$. Epochs were filtered using a sixth order zero-phase Butterworth bandpass filter with cutoff frequencies of 0.5 and $25 \mathrm{~Hz}$. Categorization was solely based on quantitative analysis. Epochs were categorized into one of six categories: normal, low-voltage, discontinuous, burst-suppression, irregular discharges, and periodic discharges. Categorization was based on four features: continuity, burst-suppression contrast ratio, frequency of epileptiform discharges, and regularity epileptiform of discharges. Continuity was defined as the fraction of signals free of "suppressions" ${ }^{8}$. Suppressions were defined 
as segments with duration of at least $0.5 \mathrm{~s}$ with all amplitudes below $10 \mu \mathrm{V}$. The burst-suppression contrast ratio was calculated if continuity was $10-90 \%$ and was defined as the power ratio between "bursts" and "suppressions". Quantitative values were derived per channel, after which the median value was calculated. Discharges were detected using the algorithm described in ${ }^{8}$. Only generalized discharges, occurring in more than 9 channels simultaneously, were taken into account. If the detected discharge frequency exceeded $0.5 \mathrm{~Hz}$, an irregularity index was calculated, defined as the standard deviation of the inter-discharge intervals divided by the mean inter-discharge interval. Epochs were classified as normal if continuity was higher than $90 \%$ and the discharge frequency was below $0.5 \mathrm{~Hz}$. Epochs with continuity below $10 \%$ were classified as low-voltage. Epochs with $10-90 \%$ continuity were classified as burst-suppression if the burst-suppression contrast ratio was 3.5 or higher, and as discontinuous if the burst-suppression contrast ratio was lower than 3.5. In case of epileptiform discharges with frequency of $0.5 \mathrm{~Hz}$ or higher, epochs were either classified as irregular discharges or as periodic discharges, depending on the irregularity parameter. Figure 6.2, in the results section, shows detailed criteria, including representative examples of clinical data and simulated EEG for each of the categories.

In order to facilitate a direct comparison with clinical EEG data, simulated EEG was filtered and categorized using the same algorithm. Simulated signals were treated as if they were single channel representations of EEG. Therefore, all detected suppressions and discharges in the simulated data were treated as if they were generalized.

\subsection{Results}

\subsubsection{Patients}

A total of 155 patients were included. Seventy-one had a good neurological outcome (CPC 1-2), 84 had a poor neurological outcome (CPC 3-5) after 6 months. Table 1 shows baseline characteristics of the included patients. As expected, patients with a good neurological outcome were younger ( 61 vs. 66 years, $p=0.025$ ), less often had a noncardiac cause of the arrest ( $6 \%$ vs. $26 \%, p<0.001$ ), and more often ventricular fibrillation (VF) as initial cardiac rhythm ( $93 \%$ vs. $60 \%, \mathrm{p}<0.001)$. Patients with good outcomes had higher sedative requirements with propofol ( $3.24 \mathrm{vs} 2.71 \mathrm{mg} / \mathrm{kg} / \mathrm{h}, \mathrm{p}=0.004)$, fentanyl (1.94 vs. $1.53 \mu \mathrm{g} / \mathrm{kg} / \mathrm{h}, \mathrm{p}=0.002$ ), and remifentanil (7.35 vs. $4.29 \mu \mathrm{g} / \mathrm{kg} / \mathrm{h}, \mathrm{p}=0.033$ ). Bilaterally absent N20 responses on a median nerve SSEP were found in $38 \%$ of patients with a poor outcome, and in none of the patients with a good outcome. There was no statistically significant difference between patients with good and poor outcomes with respect to gender, location of cardiac arrest, treatment with hypothermia, and EEG start or end times.

\subsubsection{Evolution of clinical EEG data}

Figure 6.3 schematically summarizes the EEG evolution of all patients. A few representative cases are shown in Figure 6.4. We have grouped patients according to similarities in their evolutional patterns. In 117 cases (75\%) the initial EEG was low-voltage. The largest subset (Figure 6.3A) includes 87 patients. These cases fit into a sequence from low-voltage, via discontinuous to normal EEG. Note that $63(72 \%)$ of these cases had a good outcome, and that the chance of a good outcome was higher if there was an early 


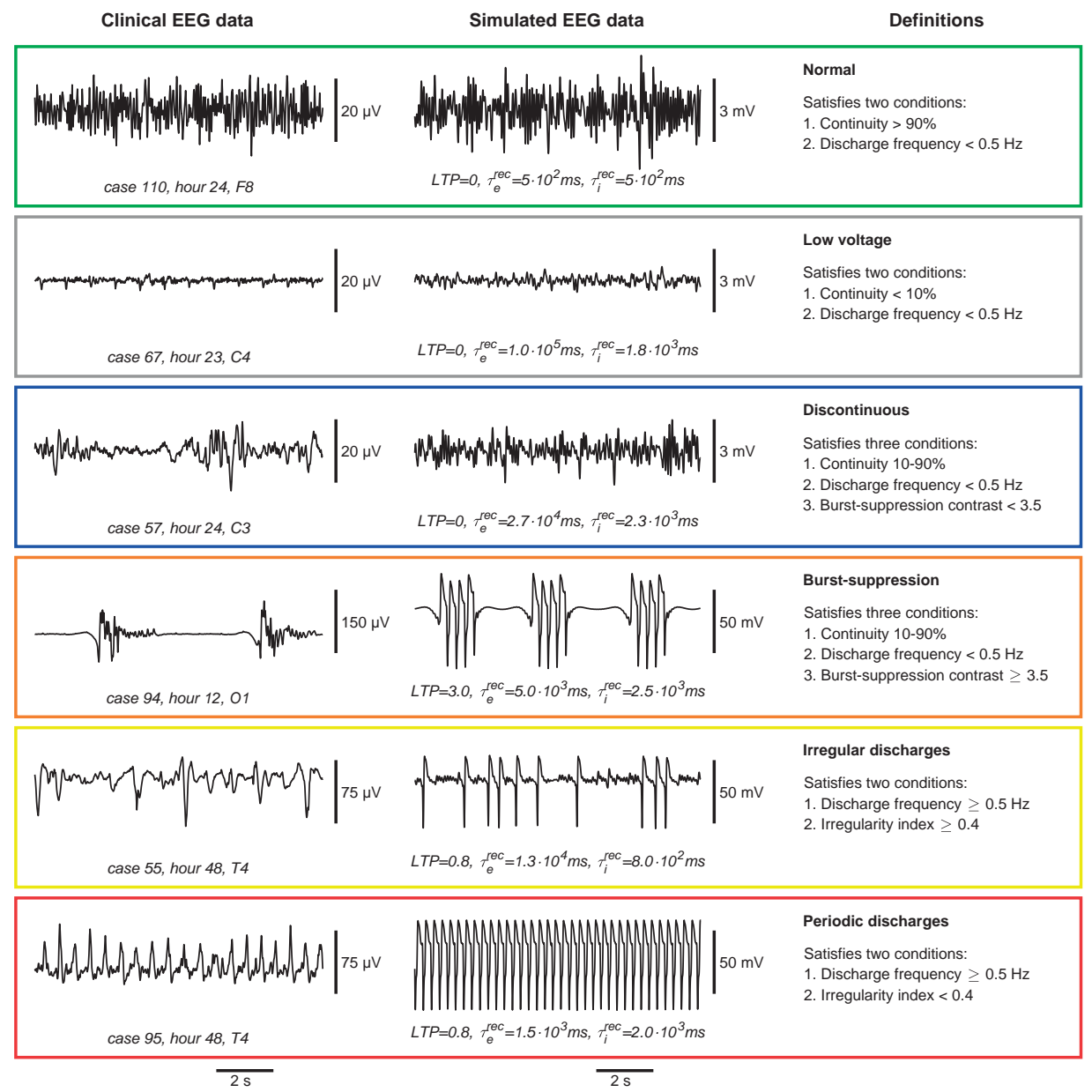

Figure 6.2: Overview of category definitions, including representative examples of clinical and simulated EEG data. The first column shows 10-second, one channel representations of clinical EEG data. Below each sample, the case number, hours since cardiac arrest, and bipolar derivation are shown. For each example, the best possible bipolar derivation was chosen based on visual inspection. The second column shows representative simulated EEG patterns. The parameters used are shown below each simulation. The third column provides category definitions. Colors shown correspond to the colors used in Figures 6.3-6.6. Note that we allowed for discharges of frequency $<0.5 \mathrm{~Hz}$ to occur in normal, low-voltage, or discontinuous patterns. 
Table 6.1: Baseline characteristics of the patients included.

\begin{tabular}{lccr}
\hline & $\begin{array}{c}\text { Poor outcome } \\
\text { (CPC 3-5) }\end{array}$ & $\begin{array}{c}\text { Good outcome } \\
\text { (CPC 1-2) }\end{array}$ & P-value \\
\hline Number of patients & $71(46 \%)$ & $84(54 \%)$ & \\
Age & $61(29-88)$ & $66(21-86)$ & 0.025 \\
Female & $21(30 \%)$ & $26(31 \%)$ & 0.850 \\
OHCA & $64(90 \%)$ & $67(80 \%)$ & 0.080 \\
Noncardiac cause & $4(6 \%)$ & $19(26 \%)$ & $<0.001$ \\
VF as initial cardiac rhythm & $63(93 \%)$ & $46(60 \%)$ & $<0.001$ \\
Treated with hypothermia $\left(33^{\circ} \mathrm{C}\right)$ & $71(100 \%)$ & $83(99 \%)$ & 0.360 \\
Patients treated with propofol & $70(99 \%)$ & $82(98 \%)$ & 0.669 \\
$\quad$ Max. propofol rate $(\mathrm{mg} / \mathrm{kg} / \mathrm{h})$ & $3.24(0.24-7.54)$ & $2.71(0.89-7.00)$ & 0.004 \\
Patients treated with midazolam & $17(24 \%)$ & $18(21 \%)$ & 0.710 \\
$\quad$ Max. midazolam rate $(\mu \mathrm{gg} / \mathrm{kg} / \mathrm{h})$ & $65(27-125)$ & $69(29-143)$ & 0.739 \\
Patients treated with fentanyl & $57(80 \%)$ & $63(75 \%)$ & 0.430 \\
$\quad$ Max. fentanyl rate $(\mu \mathrm{gg} / \mathrm{kg} / \mathrm{h})$ & $1.94(0.67-3.33)$ & $1.53(0.63-4.71)$ & 0.002 \\
Patients treated with remifentanil & $13(18 \%)$ & $18(21 \%)$ & 0.630 \\
$\quad$ Max. remifentanil rate $(\mu \mathrm{\mu g} / \mathrm{kg} / \mathrm{h})$ & $7.35(2.50-14.7)$ & $4.29(1.11-13.3)$ & 0.033 \\
Median nerve SSEP & $26(37 \%)$ & $63(75 \%)$ & $<0.001$ \\
$\quad$ Bilaterally absent $\mathrm{N} 20 \mathrm{response}$ & $0(0 \%)$ & $24(38 \%)$ & $<0.001$ \\
EEG start time (hours) & $6(2-11)$ & $6(3-11)$ & 0.795 \\
EEG end time (hours) & $68(27-142)$ & $66(5-223)$ & 0.661 \\
\hline
\end{tabular}

Numbers are displayed as mean (range), unless otherwise indicated. P-values were calculated with independent samples t-test for continuous variables and with $\chi^{2}$-test for binary variables. EEG start and end times are relative to the time of resuscitation. OHCA: out of hospital cardiac arrest. 
transition from low-voltage to a discontinuous and normal EEG. Cases in Figures 6.3B and $6.3 \mathrm{C}$ evolve from low-voltage to discontinuous EEG and, at some point to epileptiform discharges. Figure 6.3B includes patients with only irregular discharges, Figure $6.3 \mathrm{C}$ also includes patients with periodic discharges. Eight cases (40\%) in Figure 6.3B had a good outcome, while none of the patients in Figure $6.3 \mathrm{C}$ survived. For cases in Figures 6.3D and 6.3E the common transition is from low-voltage to burst-suppression EEG. Cases in Figure 6.3D do, at some point in time, improve beyond burst-suppression, and cases in Figure 6.3E do not evolve to other patterns. None of the cases in Figures 6.3D and 6.3E survived. Figure 6.3F includes cases that showed only a low-voltage EEG. None of these 11 patients had a good outcome. Note that, for all groups together, 33 of 34 patients with a 'normal' EEG within 30 hours after cardiac arrest had a good outcome. Case 143, who died as a result of respiratory problems, is the only exception. Further note that all 14 patients with no other EEG pattern than low-voltage in the first 35 hours had a poor outcome.

\subsubsection{Simulations}

Within the allowed range of parameters, the model generates all of the six categories of EEG patterns listed in Figure 6.2. The category of EEG pattern depends strongly on the synaptic recovery time constants, LTP-factor, and propofol dose. Figure 6.5A shows the spectrum of possible EEG patterns for various LTP-factors, with and without administration of propofol. The parameter planes are composed of the recovery time constants for excitatory synapses $\left(\tau_{e}^{r e c}\right)$ and inhibitory synapses $\left(\tau_{i}^{r e c}\right)$, for a fixed value of the LTP-factor. When time progresses and energy supply is restored, $\tau_{e}^{r e c}$ and $\tau_{i}^{r e c} \mathrm{c}$ will eventually recover towards their baseline values in the lower left corner. Beceause we assume that excitatory synapses are metabolically more severely affected than inhibitory synapses, only the non-shaded areas $\left(\tau_{e}^{r e c}>\tau_{i}^{r e c}\right)$ are considered physiologically plausible. Besides, parameter values in the shaded areas $\left(\tau_{e}^{r e c}<\tau_{i}^{r e c}\right)$ did not lead to plausible patterns and transitions.

The introduction of LTP changes the range of possible EEG patterns. With LTP=0, the EEG will be normal when the synaptic recovery time constants $\tau_{e}^{r e c}$ and $\tau_{i}^{r e c}$ are close to their baseline values. For larger values of the synaptic recovery time constants, discontinuous or low-voltage patterns are possible, as well. For increasing LTP-factor, subsequently, irregular discharges, periodic discharges, burst-suppression, and low-voltage EEG move into the spectrum of possible EEG patterns. This indicates that for higher LTP-values it becomes more likely for the EEG to be in one of these pathological states. If propofol is added, effects of the increased LTP-factor are partially or completely reversed. This means, for example, that in case of periodic discharges, adding propofol results in a normal or discontinuous EEG. However, if the LTP-factor does not change during treatment, these pathological patterns will reappear after propofol treatment has ended.

Figure 6.5B shows representative examples of the simulated EEG patterns. Note the difference between patterns $2 a$ and $2 b$, both classified as low-voltage. In example $2 a$, from the lower right area of the parameter space, there is still some visible activity. A closer inspection of the unfiltered signals indicates that this pattern results from hyperpolarization, i.e. the mean excitatory membrane potential $V_{e}(t)$ is below its equilib- 
A Sequence: low voltage - discontinuous - continuous

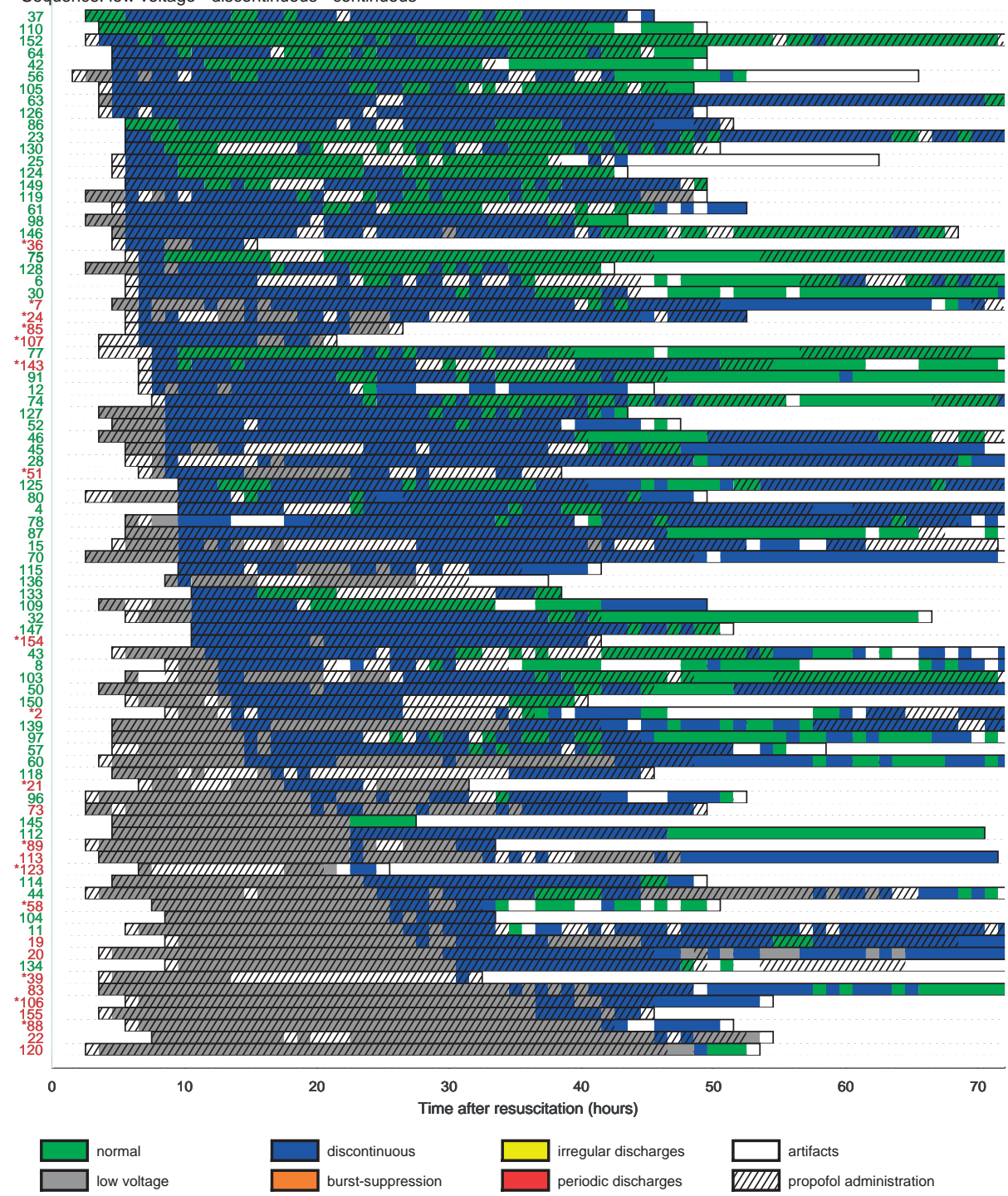

Figure 6.3: Evolution of clinical EEG data (part 1). Each row represents one case, and is preceded by the case number. Cases with good outcomes are shown in green, cases with poor outcomes in red. Data points are shown per hour, and are based on quantitative analysis of a 5-minute artifact-free epoch. Only data of the first 72 hours after cardiac arrest is shown here. Hatched areas indicate that EEG was recorded during propofol administration. Cases have been grouped by common characteristics in the evolution of their EEG. Within groups, cases have been sorted by the timing of the first non-low-voltage EEG pattern. Cases numbers preceded by “*' died as a result of a second cardiac arrest, respiratory problems, or hemodynamic instability. In all other cases who died ( $C P C=5)$, life-supporting treatment was withdrawn, at least partially based on the (suspected) poor neurological prognosis. A: cases with EEG sequence from low-voltage, via discontinuous to normal. 
B Sequence: low voltage - discontinuous - (continuous) - irregular discharges

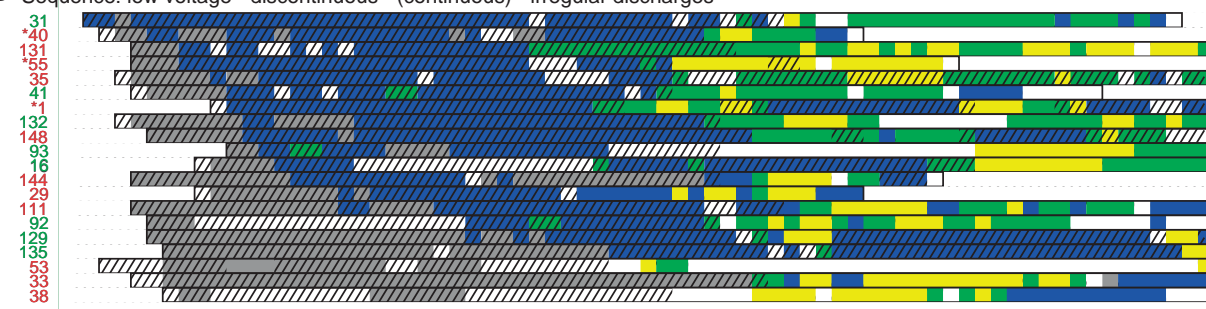

C Sequence: low voltage - discontinuous - other - periodic discharges

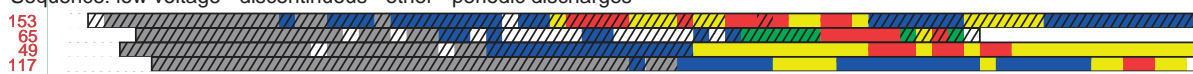

D Sequence: low voltage - burst-suppression - other

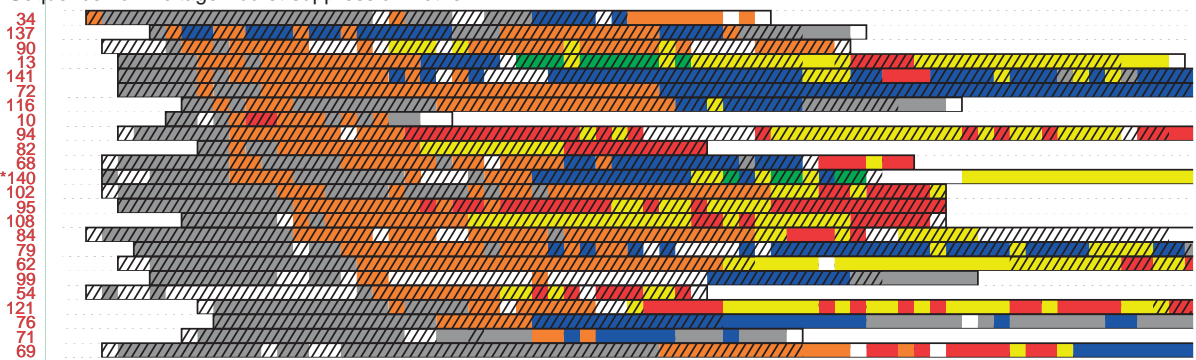

E Sequence: low voltage - burst-suppression - low voltage

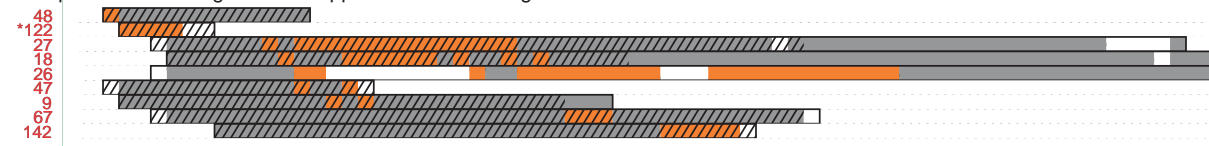

F Sequence: low voltage only

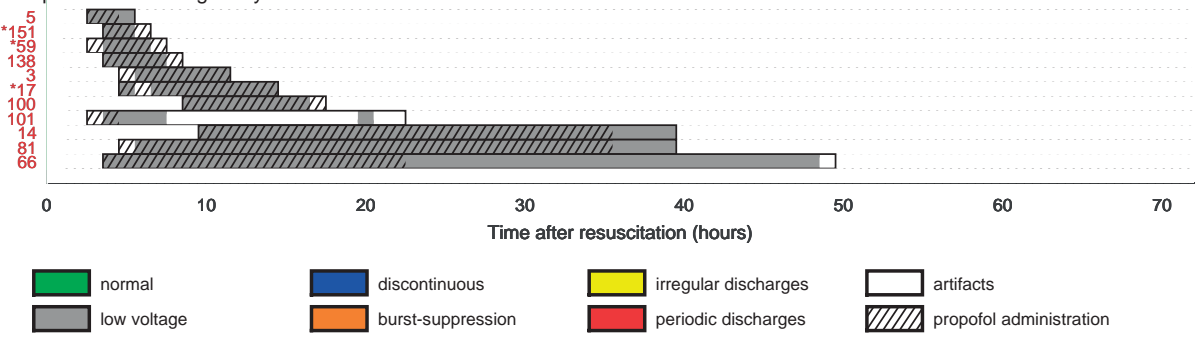

Figure 6.3: Evolution of clinical EEG data (part 2). Each row represents one case, and is preceded by the case number. Cases with good outcomes are shown in green, cases with poor outcomes in red. Data points are shown per hour, and are based on quantitative analysis of a 5-minute artifact-free epoch. Only data of the first 72 hours after cardiac arrest is shown here. Hatched areas indicate that EEG was recorded during propofol administration. Cases have been grouped by common characteristics in the evolution of their EEG. Within groups, cases have been sorted by the timing of the first non-low-voltage EEG pattern. Cases numbers preceded by '*' died as a result of a second cardiac arrest, respiratory problems, or hemodynamic instability. In all other cases who died ( $C P C=5)$, life-supporting treatment was withdrawn, at least partially based on the (suspected) poor neurological prognosis. B: cases evolving from low-voltage, via discontinuous EEG to irregular discharges. C: cases evolving from low-voltage, via discontinuous EEG to periodic discharges. D: cases evolving from low-voltage, via burst-suppression, to other patterns. E: cases with sequence low-voltage - burst-suppression - low-voltage. F: cases in whom no other pattern than low-voltage EEG was detected. Note that midazolam was used as sedative medication instead of propofol in case 26 from hour 6 to 72 , and in case 101 from hour 5 to 22. 


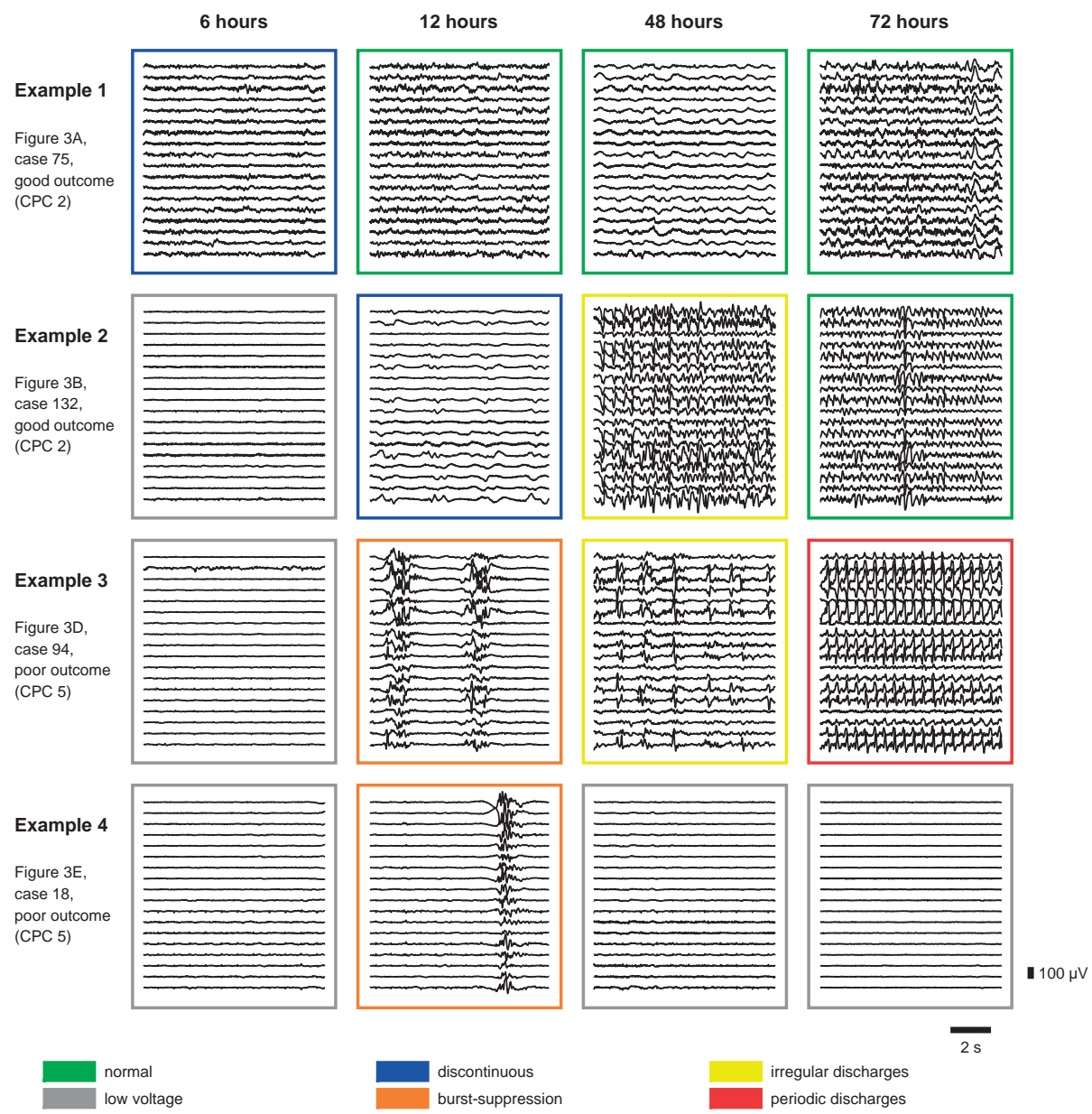

Figure 6.4: Representative examples of the evolution of clinical EEG-data. The first example (case 75, Figure $6.3 \mathrm{~A}$ ) readily improves from a discontinuous to a continuous, diffusely slowed pattern and thereafter remains continuous throughout the recording. The second example (case 132, Figure 6.3B), evolves from low-voltage to a continuous pattern and then shows a transient period with irregular discharges, a few hours after treatment with propofol was stopped. The third example (case 94, Figure 6.3D) is initially isoelectric and evolves via burst-suppression with identical bursts to generalized period discharges. The last example (case 18, Figure 6.3E) evolves from isoelectric to burst-suppression and eventually to a low-voltage EEG. 
A
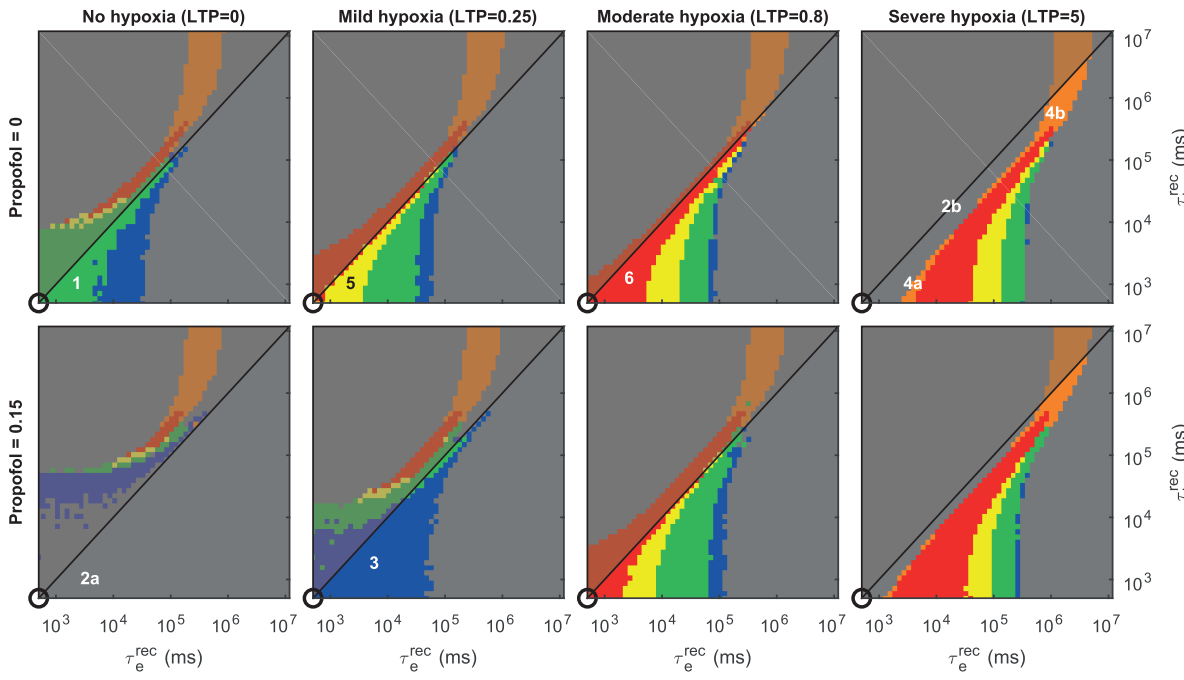

\section{$10^{7}$}

$10^{6}$

$\tau_{\mathrm{e}}^{10^{\mathrm{rec}}(\mathrm{ms})}$

irregular discharges periodic discharges

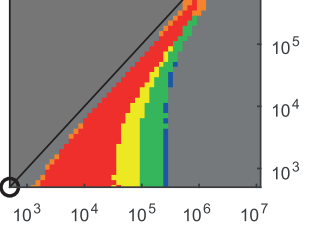

$\tau_{\mathrm{e}}^{\mathrm{rec}}(\mathrm{ms})$

$\square$ nonplausible range

O baseline parameters

B

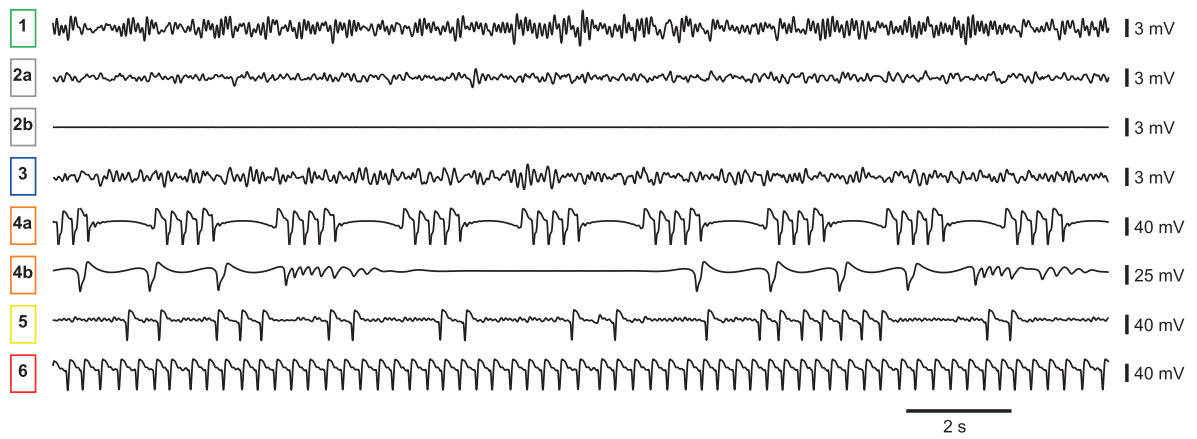

Figure 6.5: Overview of the simulations for different scenarios, with LTP ranging from LTP $=0$ to LTP=5, and with and without propofol. A: Overview of possible EEG patterns in various regions of the parameter space. The consecutive columns represent an increasing grade of anoxic injury, reflected by increasing LTP-values from left to right. The upper row represents the situation without propofol, the lower row the situation with a moderate dose ( 0.15 units) of propofol. Only the non-shaded area, where excitatory neurotransmission is more severely affected than inhibitory neurotransmission, is considered physiologically plausible. The numbers in the subplots refer to the examples in panel B. The black circle in the bottom left corner is put around the baseline value of the synaptic recovery time constants. B: Representative examples of simulated EEG patterns. 
rium value. In example $2 b$, generated in the upper left area of the parameter space, EEG activity is almost completely suppressed. Here, low-voltage EEG results from depolarization, i.e. the mean excitatory membrane potential $V_{e}(t)$ is (far) above its equilibrium value. Further note that "discontinuous" patterns in the simulations have no suppressions, like the clinical example in Figure 6.2, but are rather an amplitude intermediate between low-voltage and normal EEG patterns.

\subsubsection{Temporal evolution of simulated EEG patterns}

In the following, we will relate the possible evolution of simulated EEG to the evolutional pathways of clinical data as shown in Figure 6.3. We assume that briefly after the anoxic event, short-term synaptic recovery time constants $\tau_{e}^{r e c}$ and $\tau_{i}^{r e c}$ are significantly increased. When synapses recover from the anoxic injury, these time constants will slowly return to their baseline values, indicated by the black circles in Figure 6.5A. The EEG will therefore always evolve in this direction, if we ignore effects of permanent failure of synapses or neural cell death.

Figure 6.6 shows four possible evolutional trajectories of the EEG, corresponding to the subsets of clinical EEG data in Figure 6.3. The observations in clinical EEG data suggest that in most cases the initial EEG pattern is low-voltage. Therefore, all chosen pathways start in a low-voltage area. Pathway A leads to the sequence low-voltage - discontinuous - normal. This pathway is most likely to occur without LTP $($ LTP $=0)$. Pathway $B$ is similar to pathway A, and eventually the EEG displays irregular discharges. This pathway requires a slightly higher LTP value $(L T P=0.25)$. For a moderate LTP-value (0.8), evolution to periodic discharges is possible (Pathway $C$ ). Pathways $D$ and $E$ both include the transition from low-voltage to burst-suppression EEG. This transition is only possible for high LTP-values (LTP $\geq 2$ ). Note that burst-suppression can only occur in case of high values of the synaptic recovery time constants $\tau_{e}^{r e c} \mathrm{c}$ and $\tau_{i}^{r e c}$. This indicates that both aggravated short-term synaptic depression and potentiation of excitatory neurotransmission are required for burst-suppression patterns. See Appendix B for a mathematical description (in terms of bifurcations) of the transitions between the simulated EEG patterns.

\subsubsection{Comparison between clinical data and simulations}

If we assume that both the LTP-factor in simulations and CPC-scores in patients are measures for the severity of hypoxic injury, there are striking similarities between the simulations and the clinical EEG data. Patients had the highest (75\%) chance for a good neurological outcome if their EEG evolved in the sequence low-voltage - discontinuous - normal (Figure 6.3A). In the model simulations, this type of evolution was only possible for low or absent LTP. Patients who evolved to irregular discharges from a discontinuous or normal EEG (Figure 6.3B) had a moderate chance of a good outcome (40\%). In the model simulations, this evolutional pathway was most likely to occur for intermediate LTP values. None of the patients with periodic discharges (Figure 6.3C), or patients who evolved from low-voltage to burst-suppression patterns (Figures 6.3D and 6.3E) had a good outcome. In the simulations, these patterns were only possible for high or very high LTP-factors.

Figure 6.3 suggests that not only the sequence of EEG patterns determines the outcome, 
A

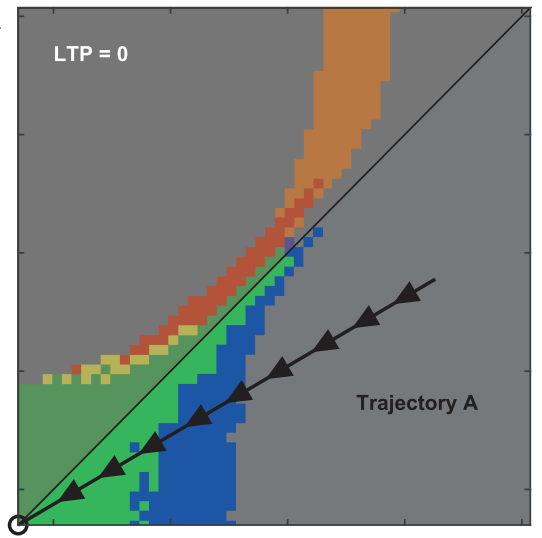

C

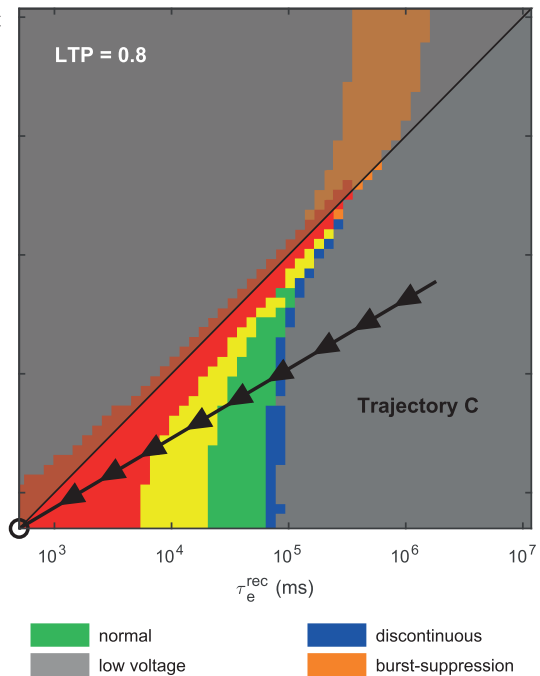

B

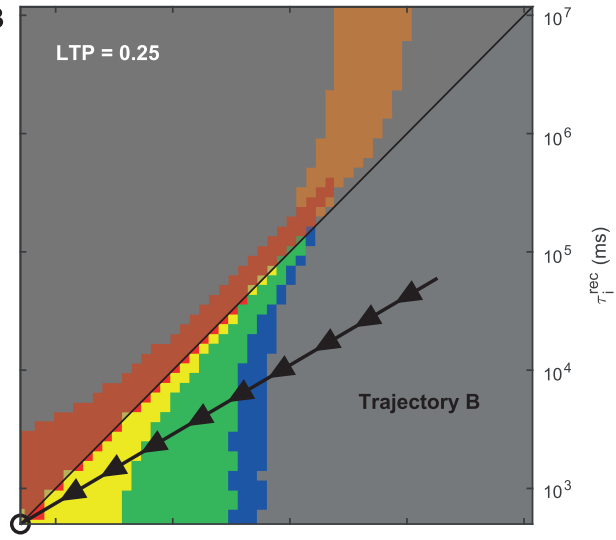

D

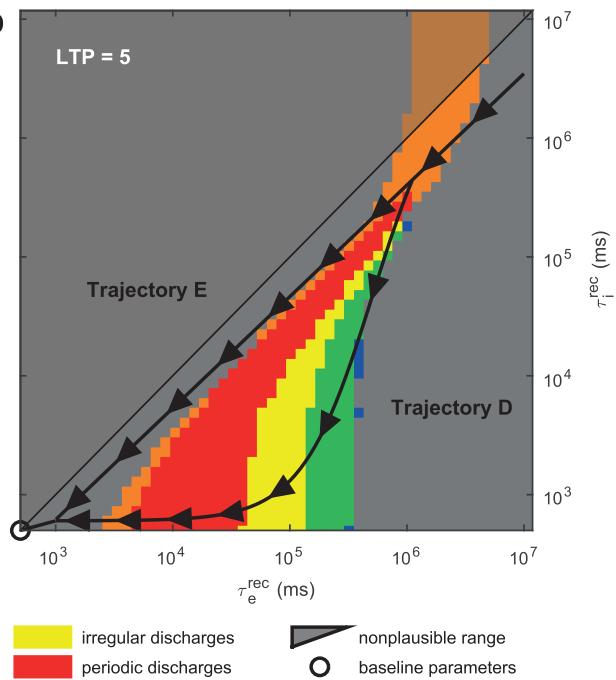

Figure 6.6: Overview of the simulations for different scenarios, with LTP ranging from LTP $=0$ to $L T P=5$, and with and without propofol. A: Overview of possible EEG patterns in various regions of the parameter space. The consecutive columns represent an increasing grade of anoxic injury, reflected by increasing LTP-values from left to right. The upper row represents the situation without propofol, the lower row the situation with a moderate dose ( 0.15 units) of propofol. Only the non-shaded area, where excitatory neurotransmission is more severely affected than inhibitory neurotransmission, is considered physiologically plausible. The numbers in the subplots refer to the examples in panel B. The black circle in the bottom left corner is put around the baseline value of the synaptic recovery time constants. B: Representative examples of simulated EEG patterns. 
but also the speed by which the EEG evolves through the transitions. From Figure 6.3, for example, follows that patients more likely had a poor outcome if they spent more time in low-voltage EEG. In terms of the model, there are two possible explanations. The first possibility is that the initial synaptic time constants in these patients are far away from their baseline values $\left(\tau_{e}^{r e c} \gg \tau_{e}^{r e c}, 0\right.$ or $\left.\tau_{i}^{r e c} \gg \tau_{i}^{r e c}, 0\right)$, indicating that the synaptic metabolism initially is severely affected. The second possibility is that the rate of synaptic recovery in these patients (i.e. the speed of decay of $\tau_{k}^{r e c}$ towards $\tau_{k}^{r e c}, 0$ ) is slower.

Finally, it was not possible to categorize patients from Figure 6.3F into one of the evolutional pathways of the model. Their low-voltage patterns can either be the result of hyperpolarization, (indicating mild hypoxic injury), depolarization (indicating severe anoxic injury), or irreversible synaptic failure (also indicating severe anoxic injury). Regarding the fact that all these patients had a poor neurological outcome, depolarization and irreversible synaptic failure are most likely.

\subsection{Discussion}

In this study, we used a mean field computational model to explain pathological EEG patterns for various grades of severity of postanoxic encephalopathy. The model incorporates mechanisms that modify the dynamics of synapses. It successfully reproduces commonly observed EEG patterns such as the physiological alpha rhythm, burstsuppression patterns, irregular and periodic discharges, and low-voltage EEG. In addition, the model correctly predicts some of the common evolutionary pathways of the EEG. In situations with mild hypoxic injury, reflected by a low LTP-factor, it correctly predicts the transition from low-voltage EEG, via discontinuous patterns, to normal EEG patterns. For severe injury, reflected by a high LTP-factor, it predicts the transition from low-voltage patterns to burst-suppression patterns and periodic discharges, without improvement to normal EEG patterns. This indicates that anoxic long-term potentiation of excitatory neurotransmission and a transient aggravation of short-term synaptic depression are plausible pathophysiological mechanisms of postanoxic encephalopathy. Furthermore, our findings support the notion that excitatory synapses are more severely affected by anoxic injury than inhibitory synapses.

Although some of the observed patterns, like periodic discharges and burstsuppression, have been simulated successfully before ${ }^{17,29}$, the dynamics of EEG evolution in postanoxic encephalopathy, including typical transitions between various EEG patterns, were never addressed.

\subsubsection{Role of short-term synaptic depression}

Previous research indicates that aggravated short-term synaptic depression is most likely the result of presynaptic mechanisms, such as a dysfunction of plasma membrane Ca2+-extrusion systems ${ }^{19}$ and a disturbed undocking of synaptic vesicles attributable to impaired phosphorylation ${ }^{20}$. Although a depletion of ATP could account for these effects, this is not likely. Simultaneous measurements of EEG and ATP levels in rat brains indicate that ATP level recovery is much faster than functional recovery as measured with $\mathrm{EEG}^{34,35}$. However, some of the amino acid levels associated with the citric acid cycle may remain abnormal for a prolonged period ${ }^{34}$ and 
secondary mitochondrial failure may occur ${ }^{36}$. Another potential mechanism is the failure of neural protein synthesis. In animal experiments, it has been shown that protein synthesis recovers on a time scale of 24 hours after the anoxic event ${ }^{37}$, which is essentially the same time scale on which the EEG recovers.

Because the net effect of short-term synaptic depression is a decrease of postsynaptic currents (PSPs), other mechanisms that decrease PSPs, such as a decrease in the number of postsynaptic neurotransmitter receptors, may also lead to some of the effects observed. Low-voltage patterns, for example, can be explained by such a mechanism. However, short-term synaptic depression has the advantage that it also facilitates periodic patterns, by means of its feedback-loop ${ }^{29,38,39}$. Our findings indicate that excitatory synapses are more strongly affected by effects of activity-dependent synaptic depression than inhibitory synapses. Simulations with equal recovery times for excitatory and inhibitory synapses, or with longer recovery times for inhibitory synapses, did not produce any plausible results.

The model observation that increasing severity of ischemic damage results in a slower restoration of background continuity is in line with previous findings. In studies on the prognostic value of continuous EEG in postanoxic encephalopathy it was found that continuous EEG activity within 12 hours after cardiac arrest indicates a good outcome, whereas outcome is always poor is there if still no measurable EEG activity after 24 hours ${ }^{1}$. Note that these findings on timing of EEG patterns in relation to outcome differ slighly from our results, as we used a stricter, quantitative definition for continuity of the EEG.

\subsubsection{Potentiation of excitatory neurotransmission}

Our results indicate that anoxic long-term potentiation of excitatory synapses is a plausible mechanism underlying certain pathological EEG patterns observed in postanoxic encephalopathy. It is compatible with the apparent network hyperexcitability in patients with severe postanoxic encephalopathy, reflected by a frequent observation of epileptiform discharges ${ }^{23,24}$. Since anoxic long-term potentiation is independent of $\mathrm{Ca}^{2+}$ - dependent presynaptic neurotransmitter release, there is no paradox between synaptic depression and a rise in extracellular glutamate levels ${ }^{40,41}$. For low LTP-values it appears that recovery is still possible. For example, in Figure 6.3B, some patients with irregular discharges improved to a normal EEG and had a good outcome. However, our clinical EEG data suggest that some evolutionary pathways always lead to a poor outcome. For example, in our dataset none of the patients who developed burstsuppression patterns or periodic discharges survived. This suggests that there may be an LTP threshold value that always leads to a poor outcome, if exceeded. This can be explained by the fact that LTP is a potential cause of secondary neural cell death, for example as a result of excessive postsynaptic calcium influx ${ }^{27}$.

On the time scales considered in this work, we assumed the LTP of excitatory neurotransmission to be permanent and static. If LTP does not cause excitotoxicity leading to secondary neural death, it is likely that its effect will be reversible. A possible mechanism is synaptic scaling ${ }^{42}$. In this form of homeostatic plasticity, excitatory synapses increase or decrease their strength in order to maintain neural firing rates. In order to 
achieve physiological postsynaptic firing rates, synaptic scaling mechanisms will normalize synaptic strength on a time scale of days. This may explain the fact that, eventually, after days, many EEG patterns observed in postanoxic encephalopathy evolve to continuous patterns, even in patients with a poor outcome ${ }^{4,5}$. However, such EEGs are typically characterized by a lower voltage and slower rhythms, presumably resulting from a significant loss of number of synapses of neurons.

\subsubsection{Effect of propofol}

The model simulations show that with the application of propofol, for low and intermediate LTP values, irregular and periodic discharges can be suppressed. By enhancement of inhibitory synaptic neurotransmission and inhibition of excitatory neurotransmission, propofol directly antagonizes the effect of long-term potentiation. This finding agees with observations in the clinical EEG data: none of the patients with good outcome, who are supposed to have little or moderate anoxic LTP, showed epileptiform discharges during propofol treatment On the other hand, propofol application was not sufficient to suppress epileptiform discharges in all number of patients with poor outcome, who presumably have a strong anoxic LTP. The model predicts that the application of propofol only temporarily suppresses periodic discharges, unless the LTP-factor decreases during treatment. It is unlikely that synaptic scaling mechanisms will account for this effect, as propofol treatment normalizes postsynaptic firing rates.

\subsubsection{Generalization of findings}

Some of the EEG abnormalities simulated with our model are not unique for postanoxic encephalopathy. For example, GPDs can also be observed in other conditions, most commonly metabolic encephalopathies, CNS infections, and acute stroke ${ }^{43}$. Since the time course of synaptic failure and LTP of excitatory neurotransmission are specific for postanoxic encephalopathy, our model findings cannot be generalized to these conditions. However, all conditions associated with GPDs readily affect synaptic neurotransmission, since it is one of the earliest events in case of energy depletion or metabolic derangements ${ }^{10}$. Therefore, it seems likely that GPDs in all these conditions result from (selective) synaptic failure, leading to an imbalance between excitatatory and inhibitory neurotransmission.

\subsubsection{Limitations}

By construction, a computational model like ours is a simplification of the neural network dynamics underlying the EEG. The main limitation of our model is that spatial aspects are not incorporated. In fact, we only simulated a single EEG channel. We find this reasonable since cerebral perfusion is diffusely affected after cardiac arrest, and EEG patterns in postanoxic coma are typically spatially homogeneous. However, particular phenomena, such as bilateral synchronization of burst suppression patterns, are not explained. Further, the lack of spatial heterogeneities may explain that the model generates a single pathological burst type, only, similar to epileptiform or identical bursts ${ }^{7}$. Using the spatio-temporal Liley model one can simulate bursts with physiological burst content, as induced by anesthetic drugs, too ${ }^{29}$.

Our model is also limited by some of the assumptions. For example, we did not incorpo- 
rate the effects of primary or secondary cell death, and neither did we take into account the effects of a disturbed ion balance on the cellular excitability. We assume this reasonable because EEG rhythms mainly represent synaptic currents ${ }^{9}$. It would, in principle, be possible to account for disturbed ion balance by adjusting the neural activation curve (S-curve) of the model, as was done for example by Zandt et al. ${ }^{44}$. The shape of the neural activation curve used in our model suggests that for high membrane potentials, the population firing rate saturates to its maximum value. However, above a certain membrane potential, a depolarization block can have significant influence on the dynamics ${ }^{45}$. We assumed for simplicity that anoxic LTP takes place immediately after cardiac arrest. This is not true, as LTP generally arises in hours. In animal experiments, the maximum increase in excitatory neurotransmission took place 5 to 10 hours after the ischemic event ${ }^{21,22}$. Finally, we assumed that all synapses are affected by hypoxia, and that all excitatory synapses or inhibitory synapses are affected in the same way, including intracortical, cortico-cortical, and thalamocortical synapses. However, experimental evidence shows that the vulnerability depends on the type of cell or synapse ${ }^{41}$. However, despite these limitations, our model faithfully simulates the main characteristic EEG patterns observed in patients with a postanoxic encephalopathy, and their temporal changes.

\subsubsection{Conclusions}

We simulated frequently observed evolving EEG patterns in postanoxic encephalopathy using a neural mean field model. The simulations indicate that aggravation of shortterm synaptic depression and potentiation of excitatory neurotransmission play a key role in the pathophysiology of postanoxic encephalopathy and recovery, as well as in the generation of EEG abnormalities. Impairment of synaptic functioning is more pronounced in excitatory than in inhibitory synapses. The model predicts that generalized periodic discharges result from a potentiation of excitatory neurotransmission and are essentially resistant to treatment.

\section{References}

1. Hofmeijer J, Beernink TMJ, Bosch FH, Beishuizen A, Tjepkema-Cloostermans MC, Van Putten MJAM. Early EEG contributes to multimodal outcome prediction of postanoxic coma. Neurology 2015; 85: 137-143.

2. Nielsen N, Wetterslev J, Cronberg T, Erlinge D, Gasche Y, Hassager C, et al. Targeted temperature management at $33 \mathrm{C}$ versus $36 \mathrm{C}$ after cardiac arrest. The New England Journal of Medicine 2013; 369: 2197-2206.

3. Tjepkema-Cloostermans MC, Hofmeijer J, Trof RJ, Blans MJ, Beishuizen A, van Putten MJAM. Electroencephalogram predicts outcome in patients with postanoxic coma during mild therapeutic hypothermia. Critical Care Medicine 2015; 43: 159-167.

4. Cloostermans MC, van Meulen FB, Eertman CJ, Hom HW, van Putten MJAM. Continuous electroencephalography monitoring for early prediction of neurological outcome in postanoxic patients after cardiac arrest: A prospective cohort study. Critical Care Medicine 2012; 40: 2867-2875.

5. Jørgensen E, Holm S. The natural course of neurological recovery following cardiopulmonary resuscitation. Resuscitation 1998; 36: 111-122.

6. Oh SH, Park KN, Shon YM, Kim YM, Kim HJ, Youn CS, et al. Continuous amplitude-integrated 
electroencephalographic monitoring is a useful prognostic tool for hypothermia-treated cardiac arrest patients. Circulation 2015; 132: 1094-1103.

7. Hofmeijer J, Tjepkema-Cloostermans MC, van Putten MJAM. Burst-suppression with identical bursts: A distinct EEG pattern with poor outcome in postanoxic coma. Clinical Neurophysiology 2014; 125: 947-954.

8. Ruijter BJ, van Putten MJAM, Hofmeijer J. Generalized epileptiform discharges in postanoxic encephalopathy: Quantitative characterization in relation to outcome. Epilepsia 2015; 56: 1845-1854.

9. Buzsáki G, Anastassiou CA, Koch C. The origin of extracellular fields and currents - EEG, ECoG, LFP and spikes. Nature Reviews Neuroscience 2012; 13: 407-420.

10. Hofmeijer J, van Putten MJAM. Ischemic cerebral damage: an appraisal of synaptic failure. Stroke 2012; 43: 607-615.

11. Coombes S. Large-scale neural dynamics: Simple and complex. Neurolmage 2010; 52: 731-739.

12. Deco G, Jirsa VK, Robinson PA, Breakspear M, Friston K. The Dynamic Brain: From Spiking Neurons to Neural Masses and Cortical Fields. PLoS Computational Biology 2008; 4: e1000092.

13. Bojak I, Liley DTJ. Modeling the effects of anesthesia on the electroencephalogram. Physical Review E 2005; 71: 1-22.

14. Robinson PA, Rennie CJ, Rowe DL. Dynamics of large-scale brain activity in normal arousal states and epileptic seizures. Physical Review E 2002; 65: 1-9.

15. Goodfellow M, Schindler K, Baier G. Intermittent spike - wave dynamics in a heterogeneous , spatially extended neural mass model. Neurolmage 2011; 55: 920-932.

16. Wendling F, Bartolomei F, Bellanger JJ, Chauvel P. Epileptic fast activity can be explained by a model of impaired GABAergic dendritic inhibition. European Journal of Neuroscience 2002; 15: 1499-1508.

17. Tjepkema-Cloostermans MC, Hindriks R, Hofmeijer J, van Putten MJAM. Generalized periodic discharges after acute cerebral ischemia: Reflection of selective synaptic failure? Clinical Neurophysiology 2014; 125: 255-262.

18. Tsodyks MV, Markram $\mathrm{H}$. The neural code between neocortical pyramidal neurons depends on neurotransmitter release probability. Proceedings of the National Academy of Sciences 1997; 94: 719-723.

19. Somjen GG. Mechanisms of early, reversible hypoxic synaptic failure. In: Ions in the Brain: normal function, seizures and stroke. Oxford University Press; 2004, p. 327-337.

20. Bolay H, Gursoy-Ozdemir Y, Sara Y, Onur R, Can A, Dalkara T. Persistent Defect in Transmitter Release and Synapsin Phosphorylation in Cerebral Cortex After Transient Moderate Ischemic Injury. Stroke 2002; 33: 1369-1375.

21. Miyazaki S, Katayama Y, Furuichi M, Kinoshita K, Kawamata T, Tsubokawa T. Post-ischemic potentiation of Schaffer collateral/CA1 pyramidal cell responses of the rat hippocampus in vivo: Involvement of N-methyl-D-aspartate receptors. Brain Research 1993; 611: 155-159.

22. Wijdicks EM, Young GB. Myoclonus status in comatose patients after cardiac arrest. The Lancet 1994; 343: 1642-1643.

23. Wijdicks E, Young G. Myoclonus status in comatose patients after cardiac arrest. The Lancet 1994; 343: 1642-1643.

24. Young GB, Gilbert JJ, Zochodne DW. The significance of myoclonic status epilepticus in postanoxic coma. Neurology 1990; 40: 1843-1848.

25. Calabresi P, Centonze D, Pisani A, Cupini LM, Bernardi G. Synaptic plasticity in the ischaemic brain. Lancet Neurology 2003; 2: 622-629.

26. Hammond C, Crépel V, Gozlan H, Ben-Ari Y. Anoxic LTP sheds light on the multiple facets of NMDA receptors. Trends in Neurosciences 1994; 17: 497-503. 
27. Szatkowski M, Attwell D. Triggering and execution of neuronal death in brain ischaemia: Two phases of glutamate release by different mechanisms. Trends in Neurosciences 1994; 17: 359-365.

28. Rossi DJ, Oshima T, Attwell D. Glutamate release in severe brain ischaemia is mainly by reversed uptake. Nature 2000; 403: 316-321.

29. Bojak I, Stoyanov ZV, Liley DT. Emergence of spatially heterogeneous burst suppression in a neural field model of electrocortical activity. Frontiers in Systems Neuroscience 2015; 9: $1-20$.

30. Liley DTJ, Cadusch PJ, Dafilis MP. A spatially continuous mean field theory of electrocortical activity. Network 2002; 13: 67-113.

31. Garcia PS, Kolesky SE, Jenkins A. General anesthetic actions on GABA(A) receptors. Current neuropharmacology 2010; 8: 2-9.

32. Khazipov R, Congar P, Ben-Ari Y. Hippocampal CA1 lacunosum-moleculare interneurons: comparison of effects of anoxia on excitatory and inhibitory postsynaptic currents. Journal of Neurophysiology 1995; 74: 2138-2149.

33. Tjepkema-Cloostermans MC, van Meulen FB, Meinsma G, Van Putten MJAM. A Cerebral Recovery Index (CRI) for early prognosis in patients after cardiac arrest. Critical Care 2013; 17 : R252.

34. Ljunggren B, Ratcheson RA, Siesjö BK. Cerebral metabolic state following complete compression ischemia. Brain Research 1974; 73: 291-307.

35. Naruse S, Horikawa Y, Tanaka C, Hirakawa K, Nishikawa H, Watari H. In vivo measurement of energy metabolism and the concomitant monitoring of electroencephalogram in experimental cerebral ischemia. Brain Research 1984; 296: 370-372.

36. Siesjö BK, Elmer E, Janelidze S, Keep M, Kristian T, Ouyang YB, et al. Role and mechanisms of secondary mitochondrial failure. Acta Neurochir Suppl (Wien) 1999; 73: 7-13.

37. Bodsch W, Barbier A, Oehmichen M, Grosse Ophoff B, Rossmann KA. Recovery of monkey brain after prolonged ischemia. II. Protein synthesis and morphological alteraltions. Journal of Cerebral Blood Flow and Metabolism 1986; 6: 15-21.

38. Liley DTJ, Walsh M. The Mesoscopic Modeling of Burst Suppression during Anesthesia. Frontiers in computational neuroscience 2013; 7: 46.

39. Tabak J, Senn W, O'Donovan MJ, Rinzel J. Modeling of Spontaneous Activity in Developing Spinal Cord Using Activity-Dependent Depression in an Excitatory Network. The Journal of Neuroscience 2000; 20: 3041-3056.

40. Ikeda M, Nakazawa T, Abe K, Kaneko T, Yamatsu K. Extracellular accumulation of glutamate in the hippocampus induced by ischemia is not calcium dependent - In vitro and in vivo evidence. Neuroscience Letters 1989; 96: 202-206.

41. Martin RL, Lloyd HGE, Cowan Al. The early events of oxygen and glucose deprivation: setting the scene for neuronal death? Trends in Neurosciences 1994; 17: 251-257.

42. Turrigiano GG. The Self-Tuning Neuron: Synaptic Scaling of Excitatory Synapses. Cell 2008; 135: 422-435.

43. Foreman B, Claassen J, Abou Khaled K, Jirsch J, Alschuler DM, Wittman J, et al. Generalized periodic discharges in the critically ill: A case-control study of 200 patients. Neurology 2012; 79: 1951-1960.

44. Zandt BJ, Visser S, Putten MJ, Ten Haken B. A neural mass model based on single cell dynamics to model pathophysiology. Journal of computational neuroscience 2014; 37: 549-568.

45. Meijer HGE, Eissa TL, Kiewiet B, Neuman JF, Schevon CA, Emerson RG, et al. Modeling focal epileptic activity in the Wilson-cowan model with depolarization block. Journal of mathematical neuroscience 2015; 5: 7.

46. Izhikevich EM. Neural Excitability, spiking and bursting. International Journal of Bifurcation and Chaos 2000; 10: 1171-1266. 


\section{A Model equations and parameters}

The spatially homogeneous Bursting Liley Model ${ }^{29}$ consists of eight coupled differential equations :

$$
\begin{aligned}
& \tau_{e} \dot{V}_{e}(t)=V_{e}^{r e s t}-V_{e}(t)+\frac{V_{e}^{e q}-V_{e}(t)}{\left|V_{e}^{e q}-V_{e}^{r e s t}\right|} I_{e e}(t)+\frac{V_{i}^{e q}-V_{e}(t)}{\left|V_{i}^{e q}-V_{e}^{r e s t}\right|} I_{i e}(t), \\
& \tau_{i} \dot{V}_{i}(t)=V_{i}^{r e s t}-V_{i}(t)+\frac{V_{e}^{e q}-V_{i}(t)}{\left|V_{e}^{e q}-V_{i}^{r e s t}\right|} I_{e i}(t)+\frac{V_{i}^{e q}-V_{i}(t)}{\left|V_{i}^{e q}-V_{i}^{r e s t}\right|} I_{i i}(t) \text {, } \\
& \ddot{I}_{e e}(t)=-\left(\gamma_{e}+\tilde{\gamma}_{e}\right) \dot{I}_{e e}(t)-\gamma_{e} \tilde{\gamma}_{e} I_{e e}(t)+\tilde{\gamma}_{e} e^{\gamma_{e} / \gamma_{e}^{0}} \Gamma_{e}(t)\left(N_{e e}^{\beta} S_{e}\left(V_{e}(t)\right)+p_{e e}(t)\right) \text {, } \\
& \ddot{I}_{e i}(t)=-\left(\gamma_{e}+\tilde{\gamma}_{e}\right) \dot{I}_{e i}(t)-\gamma_{e} \tilde{\gamma}_{e} I_{e i}(t)+\tilde{\gamma}_{e} e^{\gamma_{e} / \gamma_{e}^{0}} \Gamma_{e}(t)\left(N_{e i}^{\beta} S_{e}\left(V_{e}(t)\right)+p_{e i}(t)\right) \text {, } \\
& \ddot{I}_{i e}(t)=-\left(\gamma_{i}+\tilde{\gamma}_{i}\right) \dot{I}_{i e}(t)-\gamma_{i} \tilde{\gamma}_{i} I_{i e}(t)+\tilde{\gamma}_{i} e^{\gamma_{i} / \gamma_{i}^{0}} \Gamma_{i}(t) N_{i e}^{\beta} S_{i}\left(V_{i}(t)\right) \text {, } \\
& \ddot{I}_{i i}(t)=-\left(\gamma_{i}+\tilde{\gamma}_{i}\right) \dot{I}_{i i}(t)-\gamma_{i} \tilde{\gamma}_{i} I_{i i}(t)+\tilde{\gamma}_{i} e^{\gamma_{i} / \gamma_{i}^{0}} \Gamma_{i}(t) N_{i i}^{\beta} S_{i}\left(V_{i}(t)\right) \text {, } \\
& \dot{\Gamma}_{e}(t)=\frac{\Gamma_{e}^{r e s t}-\Gamma_{e}(t)}{\tau_{e}^{r e c}}-\rho_{e}^{d e p} S_{e}\left(V_{e}(t)\right) \Gamma_{e}(t), \\
& \dot{\Gamma}_{i}(t)=\frac{\Gamma_{i}^{r e s t}-\Gamma_{i}(t)}{\tau_{i}^{r e c}}-\rho_{i}^{d e p} S_{i}\left(V_{i}(t)\right) \Gamma_{i}(t) .
\end{aligned}
$$

Table 6.A.1 gives a physiological interpretation of the parameters and their numerical values. The functions $S_{k}\left(V_{k}\right)$ and the parameters $\gamma_{k}, \tilde{\gamma}_{k}, \Gamma_{k}^{r e s t}$, and $p_{e k}$ (with $k=e, i$ ) are not listed in the table but will be discussed below. Equations 6.A.1 and 6.A.2 determine the dynamics of the mean membrane potentials of the excitatory $\left(V_{e}\right)$ and inhibitory $\left(V_{i}\right)$ neural populations, respectively. These potentials depend on the synaptic currents $I_{n m}(t)$ with $n, m=e, i$, described by equations 6.A.3 to 6.A.6. Note that in absence of synaptic currents, the mean membrane potentials decay to their resting values $V_{e}^{\text {rest }}$ and $V_{i}^{r e s t}$, respectively. The following activation functions relate mean membrane potentials to population firing rates:

$$
\begin{aligned}
S_{e}\left(V_{e}\right) & =\frac{Q_{e}^{\max }}{1+e^{-\sqrt{2}\left(V_{e}-\mu_{e}\right) / \sigma_{e}}}, \\
S_{i}\left(V_{i}\right) & =\frac{Q_{i}^{\max }}{1+e^{-\sqrt{2}\left(V_{i}-\mu_{i}\right) / \sigma_{i}}} .
\end{aligned}
$$

The system of equations is driven by white noise input, incorporated via $p_{e e}$. We define $p_{e e}=p_{e e}^{-}+p_{e e}^{*}$, where $p_{e e}^{-}$is the mean excitatory external input and $p_{e e}^{*}$ white noise with standard deviation $s d\left(p_{e e}\right)$. The external excitatory input to inhibitory synapses $\left(p_{e i}\right)$ is constant: $p_{e i}=p_{e i}^{-}$.

For our simulations, we chose a set of baseline parameter values that result in a physiological alpha rhythm. These values were used before in $^{30}$ and ${ }^{17}$ and are shown in 
Table 6.A.1: Model parameters, their symbols, and default values $\left(\mathrm{see}^{30}\right)$

\begin{tabular}{|c|c|c|}
\hline Parameter & Symbol & Default values \\
\hline Mean soma membrane potential & $V_{e}, V_{i}$ & n.a. \\
\hline Mean resting membrane potential & $V_{e}^{\text {rest }}, V_{i}^{\text {rest }}$ & $-70 \mathrm{mV},-70 \mathrm{mV}$ \\
\hline $\begin{array}{l}\text { Mean equilibrium potential associated with } \\
\text { excitation or inhibition }\end{array}$ & $V_{e}^{e q}, V_{i}^{e q}$ & $45 \mathrm{mV},-90 \mathrm{mV}$ \\
\hline $\begin{array}{l}\text { Total number of connections that a cell of } \\
\text { type e, i receives from excitatory cells via in- } \\
\text { tracortical fibers }\end{array}$ & $N_{e e}^{\beta}, N_{e i}^{\beta}$ & 3000,3000 \\
\hline $\begin{array}{l}\text { Total number of connections that a cell of } \\
\text { type e, i receives from inhibitory cells }\end{array}$ & $N_{i e}^{\beta}, N_{i i}^{\beta}$ & 500,500 \\
\hline $\begin{array}{l}\text { Excitatory, inhibitory postsynaptic potential } \\
\text { peak amplitude }\end{array}$ & $\Gamma_{e}^{0}, \Gamma_{i}^{0}$ & $0.71 \mathrm{mV}, 0.71 \mathrm{mV}$ \\
\hline $\begin{array}{l}\text { Excitatory, inhibitory postsynaptic potential } \\
\text { rate constant }\end{array}$ & $\gamma_{e}^{0}, \gamma_{i}^{0}$ & $300 \mathrm{~s}^{-1}, 65 \mathrm{~s}^{-1}$ \\
\hline Passive membrane time constant & $\tau_{e}, \tau_{i}$ & $0.094 \mathrm{~s}, 0.042 \mathrm{~s}$ \\
\hline Excitatory, inhibitory population thresholds & $\mu_{e}, \mu_{i}$ & $-50 \mathrm{mV},-50 \mathrm{mV}$ \\
\hline $\begin{array}{l}\text { Excitatory, inhibitory population mean maxi- } \\
\text { mal firing rates }\end{array}$ & $S_{e}^{\max }, S_{i}^{\max }$ & $500 \mathrm{~s}^{-1}, 500 \mathrm{~s}^{-1}$ \\
\hline $\begin{array}{l}\text { Mean excitatory input to excitatory, inhibitory } \\
\text { cells }\end{array}$ & $p_{e e}, p_{e i}$ & $3460 \mathrm{~s}^{-1}, 5070 \mathrm{~s}^{-1}$ \\
\hline $\begin{array}{l}\text { Standard deviation of excitatory input to exci- } \\
\text { tatory cells }\end{array}$ & $\operatorname{sd}\left(p_{e e}\right)$ & $1000 \mathrm{~s}^{-1}$ \\
\hline Inhibitory input to excitatory, inhibitory cells & $p_{i e}, p_{i i}$ & $0 \mathrm{~s}^{-1}, 0 \mathrm{~s}^{-1}$ \\
\hline $\begin{array}{l}\text { Standard deviation for firing threshold in } \\
\text { excitatory, inhibitory populations }\end{array}$ & $\sigma_{e}, \sigma_{i}$ & $5 \mathrm{mV}, 5 \mathrm{mV}$ \\
\hline $\begin{array}{l}\text { Recovery time constant for activity depen- } \\
\text { dent synaptic depression }\end{array}$ & $\tau_{e}^{r e c}, \tau_{i}^{r e c}$ & $0.5 \mathrm{~s}, 0.5 \mathrm{~s}$ \\
\hline $\begin{array}{l}\text { Depletion constant for activity dependent } \\
\text { synaptic depression }\end{array}$ & $p_{e}^{d e p}, p_{i}^{d e p}$ & $0.003,0.003$ \\
\hline
\end{tabular}


Table 6.A.1. We used the mean membrane potential of the excitatory population $V_{e}(t)$ as 'EEG signal', as was done in for example ${ }^{29}$.

\section{Synaptic time constants $\left(\gamma_{k}\right.$ and $\left.\tilde{\gamma}_{k}\right)$}

Without anesthetics, the rise and decay times of the postsynaptic potentials are dictated by the baseline synaptic time constant $\gamma_{k}^{0}$. The bursting Liley model assumes that anesthetics only affect the decay rate of the inhibitory postsynaptic potential. To alter the decay rate without changing the rise rate, the time constants $\gamma_{k}$ and $\tilde{\gamma}_{k}$ are introduced, as was done for the first time in ${ }^{13}$ :

$$
\gamma_{k} \equiv \frac{\gamma_{k}^{0} \epsilon_{k}(c)}{e^{\epsilon_{k}(c)}-1}, \quad \tilde{\gamma}_{k} \equiv e^{\epsilon_{k}(c)} \gamma_{k}
$$

where $\epsilon_{k}(c)$ is a monotonically increasing function of the anesthetics concentration $c$. With this parametrization, anesthetics increases the PSP decay time, without affecting its rise time. In the limit $c \rightarrow 0$, indicating no treatment with anesthetics, $\epsilon_{k}(c) \rightarrow 0$ and $\gamma_{k}=\tilde{\gamma}_{k}=\gamma_{k}^{0}$.

In our model, we aimed to model the effects of propofol. Although $\epsilon_{k}(c)$ is quantitatively based on the effects of isoflurane ${ }^{29}$, we assumed propofol to have the same qualitative effects, since the $\mathrm{GABA}_{A}$ receptor is the most important target site of both drugs $^{31}$.

Resting value of the maximum postsynaptic potential $\left(\Gamma_{k}^{\text {rest }}\right)$

Assuming that the system of equations starts at equilibrium at $t=0$ with $V_{k}(t=0)=$ $V_{k}^{0}$ and $\Gamma_{k}(t=0)=\Gamma_{k}^{0}$,then it follows from equations (6.A.7) and (6.A.8) that the resting value of the maximum postsynaptic potential is ${ }^{29}$ :

$$
\Gamma_{k}^{r e s t}=\Gamma_{k}^{0}\left(1+\tau_{k}^{r e c} \rho_{k}^{d e p} S_{k}\left(V_{k}^{0}\right)\right) H_{k}(c),
$$

with $0 \leqslant H_{k}(c) \leqslant 1$ a Hill function depending on the concentration $c$ of anesthetic agent:

$$
\begin{aligned}
H_{e}(c) & =\frac{0.707^{2.22}}{0.707^{2.22}+c^{2.22}}, \\
H_{i}(c) & =\frac{0.79^{2.6}+0.56 c^{2.6}}{0.79^{2.6}+c^{2.6}} .
\end{aligned}
$$

This function is quantitatively based on the effects of isoflurane ${ }^{13}$. Note that EPSP amplitudes are more reduced by anesthetics than IPSP amplitudes.

For our simulations, we made a few adaptations to the equations above. First, we assumed the Hill equations 6.A.13 and 6.A.14 of isoflurane to hold qualitatively for propofol. Then, we multiplied postsynaptic potentials with $G_{e}(L T P) \geqslant 1$, a function of the long term potentiation factor LTP:

$$
\begin{aligned}
& G_{e}(l)=1+L T P, \\
& G_{i}(l)=1 .
\end{aligned}
$$


So, only excitatory synapses are affected by anoxic long term potentiation. Finally, for computational efficiency, we made the approximation $\Gamma_{k}^{0}\left(1+\tau_{k}^{r e c} \rho_{k}^{d e p} S_{k}\left(V_{k}^{0}\right)\right) \approx \Gamma_{k}^{0}$. This approximation did not qualitatively influence the simulation results. The final expression for the resting value of the maximum postsynaptic potential in our model was therefore

$$
\Gamma_{k}^{r e s t}=\Gamma_{k}^{0} H_{k}(c) G_{k}(L T P)
$$

\section{B Mathematical description of simulated EEG transitions}

The transitions between simulated EEG patterns can also be decribed mathematically. Let us consider the numbered areas in Figure 6.5A. If we start in region 2a, i.e. the hyperpolarized state, we find a stable steady state. Adding (sensory) noise yields small fluctuations. Moving towards region 3 the fluctuations become larger in amplitude. Next when moving into region 1 , the steady state exhibits a supercritical Hopf bifurcation such that stable oscillations occur in the absence of inputs. With noise the fluctuations increase in amplitude. This illustrates that the demarcations of these regions are slightly arbitrary. The transition from region 1 to 5 reveals a drastic change in excitability. In region 5, the fluctuations resemble those of region 1 , but here they may evoke an occasional spike by the noise. Moving from region 5 to 6 , the small oscillation is no longer stable through a saddle-node bifurcation. From region 6 to region $2 \mathrm{~b}$ we find again a supercritical Hopf bifurcation which results in depolarization block. In between, we find burst-suppression patterns in regions $4 \mathrm{a} / \mathrm{b}$. The specific bursting pattern is of subHopf/fold cycle-type. See also ${ }^{46}$. 



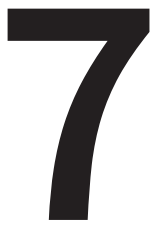

\section{Treatment of electrographic status epilepticus after} cardiopulmonary resuscitation (TELSTAR): study protocol for a randomized controlled trial

A previous version of this protocol was published as: B.J. Ruijter, M.J.A.M. van Putten, J. Horn, M.J. Blans, A. Beishuizen, A.F. van Rootselaar, J. Hofmeijer, for the TELSTAR study group, Treatment of electroencephalographic status epilepticus after cardiopulmonary resuscitation (TELSTAR): study protocol for a randomized controlled trial, Trials 2015; 15: 433. 


\section{Abstract}

Background Electroencephalographic (EEG) status epilepticus is described in $10-35 \%$ of patients with postanoxic encephalopathy after successful cardiopulmonary resuscitation and is associated with case fatality rates of $90-100 \%$. It is unclear whether these EEG patterns represent a condition to be treated with anticonvulsants to improve outcome, or an expression of severe ischemic damage, in which treatment is futile.

Methods /

TELSTAR is a multicenter clinical trial with randomized treatment Design allocation, open label treatment, and blinded endpoint evaluation (PROBE design). We aim to enroll 172 adult patients with postanoxic encephalopathy and electroencephalographic status epilepticus after successful cardiopulmonary resuscitation, admitted to the intensive care unit, in whom continuous EEG monitoring is started within 24 hours after admission. Patients are randomly assigned to either medical treatment to suppress all electrographic seizure activity, or no treatment of electroencephalographic status epilepticus. Antiepileptic treatment is based on guidelines for treatment of overt status epilepticus and is started within three hours after the diagnosis. If status epilepticus returns during tapering of sedative medication after suppression of all epileptiform activity for $2 \times 24$ hours, it will be considered refractory. The primary outcome measure is neurological outcome defined as the Cerebral Performance Category (CPC) score at three months, dichotomized into "good" (CPC 1-2 = no or moderate neurological disability) and "poor" (CPC 3-5 = severe disability, coma, or death). Secondary outcome measures include mortality and, for patients surviving up to 12 months, cognitive functioning, health related quality of life, and depression. 


\subsection{Background}

After successful cardiopulmonary resuscitation, $64-74 \%$ of patients remain unconscious at hospital arrival as a result of diffuse postanoxic encephalopathy ${ }^{1,2}$. In these patients, a spectrum of electroencephalographic (EEG) changes can be observed, reflecting varying extents of ischemic brain injury ${ }^{3}$. Electroencephalographic status epilepticus is described in $10-35 \%{ }^{3-8}$ and is strongly associated with poor outcome: case fatality was $90-100 \%$ in prospective case series, despite treatment with anticonvulsants ${ }^{4,6-10}$. Without EEG monitoring, approximately one in five cases remains undiscovered due to the absence of clinical signs ${ }^{4,11,12}$.

The diagnostic criteria for electroencephalographic status epilepticus in comatose patients with postanoxic encephalopathy are controversial ${ }^{13,14}$. The American Clinical Neurophysiology Society defines unequivocal seizures as generalized spike-wave discharges at $3 \mathrm{~Hz}$ or faster or clearly evolving discharges of any type at $4 \mathrm{~Hz}$ or faster, either focal or generalized ${ }^{15}$. However, some experts also consider other rhythmic or periodic patterns, such as generalized or lateralized periodic discharges or rhythmic delta activity as seizure activity ${ }^{4,6}$.

It is unclear whether electroencephalographic seizure patterns in patients with postanoxic encephalopathy represent a condition which can be treated with anticonvulsants to improve patients' outcome, or have to be regarded as an expression of severe ischemic damage, in which treatment with anticonvulsants would be futile ${ }^{16,17}$. Case series suggest that in patients with electroencephalographic status epilepticus, preserved brainstem reactions and EEG background reactivity are associated with a favorable outcome ${ }^{7}$. It is unclear whether treatment with anticonvulsants reduces the risk of a poor outcome in these patients and if so, how intensive this treatment should be. In the only prospective, non-randomized intervention study, a stepwise treatment up to pentobarbital induced burst suppression resulted in a good outcome of $6 \%$ of patients with clinically overt or electroencephalographic status epilepticus ${ }^{18}$. This proportion is approximately the same as reported in observational studies, irrespective of treatment ${ }^{4,6,7,10}$.

Despite the lack of evidence, most neurologists treat status epilepticus in comatose patients after cardiopulmonary resuscitation with anticonvulsants. Increased detection of electroencephalographic status epilepticus by continuous EEG monitoring has led to increased prescription of these drugs ${ }^{19,20}$. However, treatment is mostly moderately intensive. In the Netherlands, only one third of the physicians treat these patients equally intensive as those with clinically overt status epilepticus ${ }^{21}$. Both intensive antiepileptic treatment and no treatment of electroencephalographic status epilepticus are considered standard modalities, where some experts believe that treatment is useless and others that it is unethical to withhold it.

Apart from the intensity, the timing of treatment probably is an important determinant of treatment effect. Mechanisms such as excessive glutamate release are known to worsen brain damage in ongoing status epilepticus within twenty to forty minutes, even without clinical signs and despite good oxygenation ${ }^{22}$. Also, prolonged duration of status epilepticus reduces the effect of treatment, e.g. due to receptor trafficking ${ }^{23}$. In approximately a quarter of patients, the electroencephalographic status epilepticus 
starts within 24 hours after cardiopulmonary resuscitation ${ }^{5,9,12}$. In previous studies, EEG monitoring started at a median of two to three days after cardiopulmonary resuscitation, indicating that diagnosis and subsequent treatment of electroencephalographic status epilepticus started relatively late ${ }^{6,7}$.

We conclude that evidence for a beneficial effect of medical treatment of electroencephalographic status epilepticus in patients with postanoxic encephalopathy after cardiopulmonary resuscitation is insufficient. To be effective, treatment should be sufficiently intensive and initiated as early as possible after the onset of status epilepticus. Therefore, we aim to study the effect of intensive and early medical treatment of electroencephalographic status epilepticus on functional outcome of comatose patients after cardiopulmonary resuscitation in a randomized controlled clinical trial.

\subsubsection{Hypothesis}

Medical treatment of electroencephalographic status epilepticus improves outcome of patients with postanoxic encephalopathy after successful cardiopulmonary resuscitation.

\subsection{Methods / Design}

\subsubsection{Design and population}

TELSTAR is a multicenter clinical trial with two parallel groups, randomized treatment allocation, open label treatment and blinded endpoint evaluation (PROBE design). The trial has been registered in the United States National Institutes of Health Clinical Trials registry (clinicaltrials.gov, identifier NCT02056236) on February 4, 2014. The study population consists of comatose adult patients after cardiopulmonary resuscitation, admitted to the intensive care unit (ICU), with electroencephalographic status epilepticus on continuous EEG. Detailed eligibility criteria are listed in Table 7.1.

Definitions of electroencephalographic status epilepticus will be according to standardized critical care EEG terminology ${ }^{15}$. They may consist of generalized spike-wave discharges at $3 \mathrm{~Hz}$ or faster, clearly evolving discharges of any type at $4 \mathrm{~Hz}$ or faster (either generalized or focal), or periodic discharges (generalized or lateralized) at any frequency. For continuous seizure activity, the minimum duration requirement is 30 minutes. Intermittent seizures of 5 minutes and longer, recurring at least twice, with seizure-free intervals shorter than 60 minutes will also be included. EEG assessment for inclusion will ultimately be left to the discretion of the treating neurologist or clinical neurophysiologist.

\subsubsection{Deferred consent}

No consent will be obtained prior to randomization, as this procedure unnecessarily delays treatment and the possible prevention of additional brain injury. We consider it reasonable to start treatment without written informed consent, since we compare two standard treatment modalities. The treating physician will inform a patient's legal representative as soon as possible after randomization, and ask for consent for the use of anonymized clinical data and for the first telephone follow-up after 3 months. Surviving patients will be asked informed consent for participation and additional follow-up 
Table 7.1: Eligibility criteria.

\begin{tabular}{ll}
\hline Inclusion criteria & Exclusion criteria \\
\hline $\begin{array}{l}\text { Comatose patients after cardiac arrest with } \\
\text { suspected postanoxic encephalopathy }\end{array}$ & $\begin{array}{l}\text { A known history of another medical con- } \\
\text { dition with limited life expectancy (<6 } \\
\text { months) }\end{array}$ \\
Age 18 years or older & $\begin{array}{l}\text { Any progressive brain illness, such as a brain } \\
\text { tumor or neurodegenerative disease }\end{array}$ \\
$\begin{array}{l}\text { Continuous EEG with at least eight elec- } \\
\text { trodes started within 24 hours after car- } \\
\text { diopulmonary resuscitation }\end{array}$ & $\begin{array}{l}\text { Pre-admission Glasgow Outcome Scale } \\
\text { score of } 3 \text { or lower }\end{array}$ \\
$\begin{array}{l}\text { Electroencephalographic status epilepticus } \\
\text { on continuous EEG }\end{array}$ & $\begin{array}{l}\text { Reason other than neurological condition to } \\
\text { withdraw treatment }\end{array}$ \\
$\begin{array}{l}\text { Informed consent given by a legal represen- } \\
\text { tative }\end{array}$ & $\begin{array}{l}\text { Follow-up impossible due to logistic rea- } \\
\text { sons, for example not living in the Nether- } \\
\text { lands }\end{array}$ \\
\hline
\end{tabular}
${ }^{*}$ If status epilepticus is present at initiation of continuous EEG, the starting time of continuous EEG is
considered as detection time. EEG = Electroencephalography.

on long term outcome. Separate informed consent will be asked for neuropsychological examination at twelve months, if applicable. The study will be conducted according to the principles of the Declaration of Helsinki (7th revision, Fortaleza, 2013) and in accordance with the Dutch Medical Research Involving Human Subjects Act (WMO) and local guidelines.

\subsubsection{Treatment allocation}

All participating subjects receive standard best medical management according to current guidelines, including therapeutic hypothermia or controlled normothermia with sedation for 24 hours. In addition, they will be randomly assigned to either medical treatment or no treatment of electroencephalographic status epilepticus. In both groups, decisions regarding limitation or withdrawal of treatment will be done in accordance with the Dutch guideline for postanoxic coma management ${ }^{24}$. Reasons for withdrawal of treatment will be documented.

Subjects will be randomized using ALEA (Clinical Trial Center Maastricht, The Netherlands), which is an online, central randomization service. To prevent imbalance of allocated treatments, blocked randomization will be used, with a 1:1 allocation, stratified by center, and random block size ranging from 4 to 10 subjects.

\section{Intervention group: treatment of electroencephalographic status epilepticus}

Since no treatment with anticonvulsants has been proven superior to other, the choice of medication is ultimately left to the discretion of the treating neurologist, based on local protocols. However, to prevent large differences with respect to the intensity of 
treatment, recommendations are made as displayed in Figure 7.1. In summary, treatment consists of a stepwise approach, from intermittent administration of antiepileptic drugs up to treatment with barbiturates, in accordance with international guidelines for treatment of overt status epilepticus ${ }^{25,26}$. Each subsequent step is taken as soon as possible when previous steps fail to suppress epileptiform activity. The treatment objective is to suppress all epileptiform activity on the EEG during at least 24 hours. Induction of burst suppression is not obligate. If the status epilepticus returns after tapering sedative medication, the treatment procedure will be repeated during another 24 hours. If status epilepticus returns after $2 \times 24$ hours, it is considered refractory. We accept that treatment with barbiturates may lead to prolonged hospitalization of several days, due to their influence on prognostic tests.

\section{Control group: no treatment of electroencephalographic status epilepticus}

In this group, patients will be treated conform standard guidelines for treatment of comatose patients after cardiopulmonary resuscitation, without EEG based treatment of status epilepticus. Treatment to suppress clinically manifest myoclonic jerks is left to the discretion of the treating physician, also in patients surviving beyond ICU discharge.

\subsubsection{Outcome assessment}

The primary outcome measure is neurological outcome, defined as the Cerebral Performance Category (CPC) score at 3 months, dichotomized into "good" (CPC 1-2 = no or moderate neurological disability) and "poor" (CPC 3-5 = severe disability, coma, or death).

Secondary outcome measures include mortality, CPC scores at six and twelve months, length of stay on the ICU, duration of mechanical ventilation, and seizure recurrence rate within twelve months. In case of survival, additional outcome measures include quality of life after a year as measured by the Medical Outcomes Study 36-item shortform health survey (SF-36) ${ }^{27}$, depression after a year as measured by the Montgomery and Åsberg Depression Rating Scale (MADRS) ${ }^{28}$, and cognitive functioning after a year as measured by detailed neuropsychological examination. Secondary outcome measures will not be collected to test between-group differences, since the estimated number of survivors is small. These measures will be collected to thoroughly assess outcome and quality of life of survivors.

Additionally, a limited amount of data on the use of resources will be collected for analysis of cost-effectiveness, including place of residence at one year and admission in hospitals, rehabilitations centers, and nursing homes within the first year.

\subsubsection{Study procedures and data collection}

For data collection and management, the OpenClinica open source software (OpenClinica LLC and collaborators, Waltham, MA, USA) will be used. At randomization, patients receive a study number by which all data is coded. The trial coordinator and principal investigator safeguard the key to this code.

Figure 7.2 shows the study flow chart. In participating hospitals, continuous EEG monitoring is part of regular patient care and is initiated as soon as possible after admission 


\section{Step 1: antiepileptic drug + benzodiazepine}

Phenytoin: loading dose 15-20 mg/kg iv, maintenance doses $150 \mathrm{mg}$ iv twice per day *

Plus one of the following benzodiazepines (bolus + continuous infusion):

I. Lorazepam

II. Midazolam

Benzodiazepine dosing regimes should be based on national and local protocols for status epilepticus treatment.

* Use serum levels for phenytoin dose adjustment. If phenytoin is contraindicated, use either valproic acid or levetiracetam as drug of first choice. The drug of second choice can then be applied in step 2. See step 2 for recommended doses.

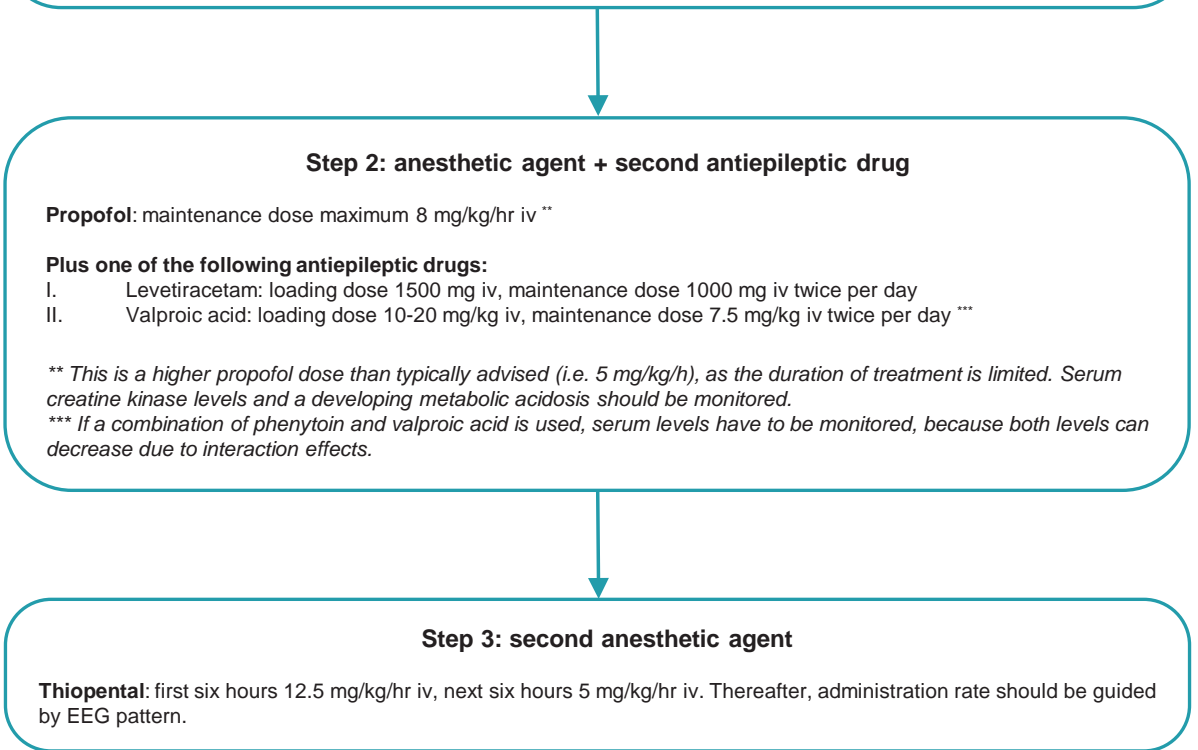

Figure 7.1: Stepwise treatment approach for electroencephalographic status epilepticus. Each consecutive treatment step is taken when previous steps have failed to suppress electroencephalographic seizure activity. After 24 hours of treatment, sedative agents (thiopental, propofol, and continuously administrated benzodiazepines) will be tapered and intermittently administrated antiepileptic drugs (phenytoin, valproic acid, and levetiracetam) will be continued. If the status epilepticus returns, the treatment procedure will be repeated during another 24 hours. If status epilepticus returns after 2 × 24 hours, it is considered refractory. EEG = Electroencephalography. 


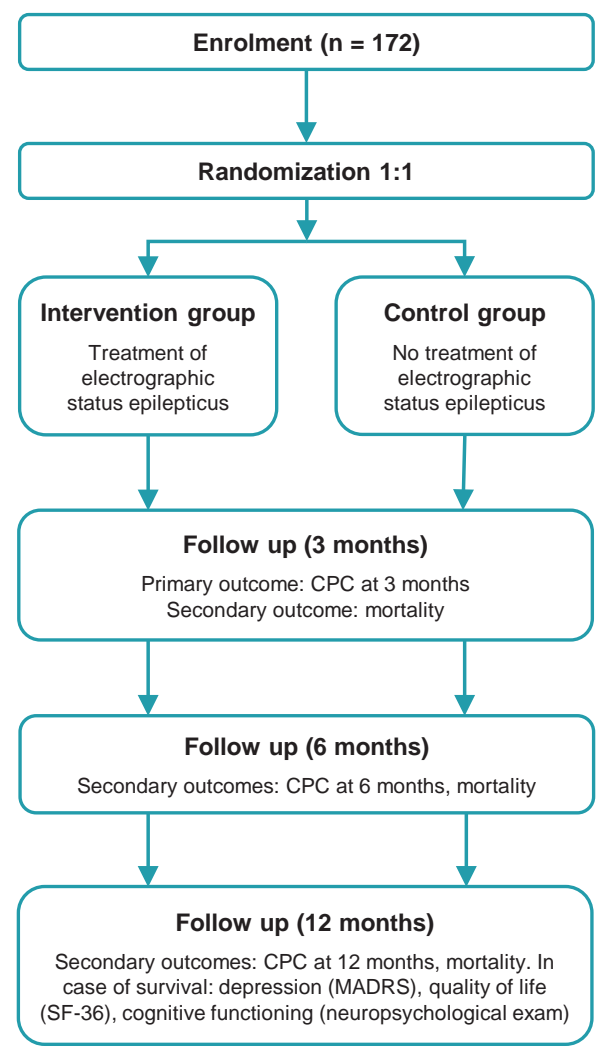

Figure 7.2: Study flow chart. CPC = Cerebral Performance Category; MADRS: Montgomery and Åsberg Depression Rating Scale; SF-36: Medical Outcomes Study 36-item short-form health survey.

to the intensive care unit. In order to detect seizure activity early, continuous EEG registrations are checked three hourly by a neurologist, clinical neurophysiologist, or clinical neurophysiology technician. For practical reasons, these checks will not take place regularly between midnight and 8 am. When patients meet the eligibility criteria and informed consent is obtained, they will be randomly allocated to one of the treatment arms. It is not possible to see the treatment allocation before the patient is randomized and registered in the study database. Neither is it possible to withdraw the patient from the database after treatment assignment. After randomization, treating physicians and patients (or their legal representatives) will be aware of the treatment assignment.

Baseline data will be obtained at randomization and include patient characteristics (age, sex, medical history, use of anticonvulsants), pre-hospital factors (cause and location of cardiac arrest, initial cardiac rhythm, time to return of spontaneous circulation), clinical parameters (Glasgow Coma Scale score, brain stem reflexes), electroencephalographic features (seizure pattern, background reactivity), target temperature, and the Acute Physiology and Chronic Health Evaluation (APACHE) score. During admission on 
the ICU, daily neurological examinations will be performed as part of regular care. Selected medication for treatment of the status epilepticus will be recorded. At ICU discharge or in case of mortality, the duration of ventilation and duration of admission will be assessed.

Follow-up will be done by means of a telephone interview by a trained investigator or research nurse, who is blinded for the allocated treatment. At three, six, and twelve months, CPC scores will be assessed. Place of residence, duration of admission in hospitals, rehabilitations centers, or nursing homes, seizure recurrence rates, use of anticonvulsants, and serious adverse events will be recorded. At twelve months, after obtaining separate informed consent, quality of life, depression, and cognitive functioning will be assessed in the local hospital.

Subjects can leave the study at any time for any reason if they wish to do so without any consequences. Also, the investigator can decide to withdraw a subject from the study for urgent medical reasons. Subjects will be replaced after withdrawal for any reason. Every attempt will be made to complete the primary follow-up in patients who are withdrawn from treatment.

\subsubsection{Statistical considerations}

The primary analysis will be a single comparison between the treatment groups with regard to the primary outcome measure at three months, according to the intentionto-treat principle. To assess the effect of treatment with anticonvulsants, the absolute risk reduction of poor outcome will be calculated, including the corresponding 95\% confidence interval. Baseline characteristics, raw distributions of the CPC score, and scores of secondary outcome measures will first be presented in a descriptive way. For secondary outcome measures, between-group differences will be analyzed by means of independent samples t-tests, Mann-Whitney tests, or Fisher exact tests, where appropriate. If necessary, multivariate regression analysis will be used to adjust for imbalances in main prognostic variables between the intervention and control groups.

Power calculations are hampered by the absence of data from randomized trials. With a presumed reduction of poor outcome from $99 \%$ to $92 \%$, alpha of $5 \%$, power of $80 \%$, and one tailed testing, 84 patients per treatment group are needed to detect superiority of treatment with anticonvulsants. An interim analysis according to O'Brien Fleming will be performed after a total of 86 enrollments. If the difference between the treatment groups at that time is significant at $p<0.00557$, the trial will be stopped because of "proof beyond reasonable doubt" that treatment with anticonvulsants is superior above treatment without anticonvulsants. To compensate for this interim analysis, two additional patients per group will be included. This indicates an intended sample size of 172 subjects.

With an estimated incidence of electroencephalographic status epilepticus of $20 \%$ in comatose patients with postanoxic encephalopathy ${ }^{3}, 860$ patients will have to be monitored with continuous EEG. With five Dutch hospitals participating at submission of this manuscript, we estimate an enrollment period of four and a half years. 


\subsubsection{Safety assessments}

Medical treatment of electroencephalographic status epilepticus may lower the high risk of death. The risk of an increase of morbidity or mortality is considered negligible. Otherwise, treatment may lead to prolonged hospitalization of several days of comatose patients that otherwise would have died. We assume this reasonable, since both antiepileptic treatment and no treatment are current standard modalities in these patients.

Adverse events will not be recorded, except those that meet the criteria for 'Serious Adverse Event'. A serious adverse event is defined as any adverse event that results in death, a life-threatening condition, inpatient hospitalization, or prolongation of existing hospitalization, or persistent or significant disability. The reporting of SAEs will be limited to events that occur during the intensive treatment period (the period in which patients are exposed to the treatment under study) and all deaths within the follow-up period of one year.

The trial is monitored by an independent Data Safety Monitoring Board (DSMB). The DSMB is chaired by a neurologist and further includes an intensivist and a biostatistician. This committee will review data for safety after every 43 enrolled patients from the study have had their primary outcome measurement. Because of the expected high proportion of patients with a poor outcome in the study population, and the consequent overlap between safety endpoints and the primary endpoint, the evaluation of safety by the DSMB at that time will be qualitative. Summary of key efficacy endpoints (primary outcomes, mortality) will be provided for a planned interim analysis after 86 enrollments.

\subsubsection{Ethical approval}

TELSTAR has received ethical approval from the Medical Research Ethics Committee Twente (Dutch: METC Twente) in December 2013 (reference: NL46296.044.13).

\subsubsection{Publication of trial results}

The trial results will be published by the members of the Executive Committee, on behalf of the TELSTAR study team. With 36 or more enrollments, local principle investigators of participating centers will be included in de primary author list. Before submission of any manuscript, all local principal investigators will have the opportunity to comment on the manuscript.

\subsection{Discussion}

We present a study protocol for a multicenter, randomized controlled trial to investigate treatment of electroencephalographic status epilepticus in patients with postanoxic encephalopathy after cardiopulmonary resuscitation. Both sides of the spectrum, e.g. antiepileptic treatment to burst suppression and no treatment at all of electroencephalographic status epilepticus are current standard modalities in these patients ${ }^{19,21}$. The benefits of antiepileptic treatment have never been investigated in a randomized controlled trial.

The focus of debate is often whether EEG patterns represent epileptic activity, that can 
be treated with anticonvulsants to improve outcome, or not ${ }^{13,14}$. In the only prospective case series of intensive antiepileptic treatment in these patients, EEG patterns of enrolled subjects were insufficiently reported ${ }^{18}$. In the current trial, we include a broad range of possibly epileptiform EEG patterns. However, with a clear definition of the distinct eligible seizure patterns, based on standardized EEG terminology ${ }^{15}$, we aim to increase insight in the effects of treatment in relation to specific patterns. Although EEG analysis prior to patient enrollment will be performed locally by the treating neurologist or clinical neurophysiologist, all EEGs will also be analyzed off-line by dedicated clinical neurophysiologists, to optimize categorization of patterns.

If beneficial at all, the optimal duration of treatment of status epilepticus in postanoxic encephalopathy is unclear. Previous studies suggest that in more than half of cases the duration of seizure activity is less than 24 hours, and in more than three quarters of cases less than 48 hours ${ }^{5,12}$. We consider ongoing status epilepticus after suppression of all epileptiform activity for $2 \times 24$ hours as refractory and argue that patients with ongoing seizure activity beyond this period are unlikely to benefit from prolonged antiepileptic treatment.

In previous, non-randomized studies concerning treatment of status epilepticus after cardiopulmonary resuscitation, diagnosis was either based on clinical signs ${ }^{29-31}$, or EEG seizure activity ${ }^{4,6,9}$, or a combination of clinical and EEG findings ${ }^{7,18}$. However, myoclonic jerks are often suppressed by sedative medication. Furthermore, myoclonia are often unrelated to EEG findings. For most neurologists, the threshold to treat patients with overt myoclonia is lower than for patients with non-convulsive electroencephalographic seizures. However, irreversible damage is probably even more likely in patients with myoclonia, since the risk of poor outcome is larger ${ }^{7}$ and neuronal necrosis is more common ${ }^{17}$. In the current study, the diagnosis of status epilepticus is EEG based, irrespective of clinical signs.

Our study protocol intends to minimize time from onset of electroencephalographic status epilepticus to start of treatment, as ongoing seizure activity may lead to additional damage ${ }^{22}$ and decreased effect of medication ${ }^{23}$. EEG monitoring will be started within 24 hours. Epileptic activity that is initially suppressed by the anticonvulsive effects of hypothermia and sedative medication will therefore be detected early. The maximum time of 3 hours since the diagnosis to obtain informed consent and commence treatment is chosen pragmatically.

Although the trial protocol contains clear recommendations, including the intensity of antiepileptic treatment in the intervention group, the diagnosis of electrographic status epilepticus and the choice of specific anticonvulsive medication are ultimately left to the discretion of the treating physician. Since we consider the pragmatic character of this difficult trial very important, we accept potentially detrimental effects of variations due to freedom of treatment. These include restricted extrapolation resulting from differences between local protocols and effect dilution resulting from treatment of overt myoclonia with anticonvulsants in the control group.

Blinding of treating physicians for treatment allocation is virtually impossible, as the administration of anticonvulsants is guided by bedside EEG measurements. However, 
outcome assessment of surviving patients is performed blinded for the treatment allocation.

Concluding, this study will be the first to evaluate the additional value of medical treatment of electroencephalographic status epilepticus for patients after successful cardiopulmonary resuscitation. Furthermore, it will provide clinical and electrographic characteristics for identifying patients that might benefit from this treatment.

\section{References}

1. Gorjup V, Radsel P, Kocjancic ST, Erzen D, Noc M. Acute ST-elevation myocardial infarction after successful cardiopulmonary resuscitation. Resuscitation 2007; 72: 379-385.

2. Hosmane VR, Mustafa NG, Reddy VK, Reese CL, DiSabatino A, Kolm P, et al. Survival and neurologic recovery in patients with ST-segment elevation myocardial infarction resuscitated from cardiac arrest. Journal of the American College of Cardiology 2009; 53: 409-415.

3. Cloostermans MC, van Meulen FB, Eertman CJ, Hom HW, van Putten MJAM. Continuous electroencephalography monitoring for early prediction of neurological outcome in postanoxic patients after cardiac arrest: A prospective cohort study. Critical Care Medicine 2012; 40: 2867-2875.

4. Legriel S, Bruneel F, Sediri H, Hilly J, Abbosh N, Lagarrigue MH, et al. Early EEG monitoring for detecting postanoxic status epilepticus during therapeutic hypothermia: a pilot study. Neurocritical Care 2009; 11: 338-344.

5. Rittenberger JC, Popescu A, Brenner RP, Guyette FX, Callaway CW. Frequency and timing of nonconvulsive status epilepticus in comatose post-cardiac arrest subjects treated with hypothermia. Neurocritical Care 2012; 16: 114-122.

6. Rossetti AO, Logroscino G, Liaudet L, Ruffieux C, Ribordy V, Schaller MD, et al. Status epilepticus: An independent outcome predictor after cerebral anoxia. Neurology 2007; 69: 255-260.

7. Rossetti AO, Oddo M, Liaudet L, Kaplan PW. Predictors of awakening from postanoxic status epilepticus after therapeutic hypothermia. Neurology 2009; 72: 744-749.

8. Rundgren M, Rosén I, Friberg H. Amplitude-integrated EEG (aEEG) predicts outcome after cardiac arrest and induced hypothermia. Intensive Care Medicine 2006; 32: 836-842.

9. Hofmeijer J, Tjepkema-Cloostermans MC, Blans MJ, Beishuizen A, van Putten MJAM. Unstandardized Treatment of Electroencephalographic Status Epilepticus Does Not Improve Outcome of Comatose Patients after Cardiac Arrest. Frontiers in Neurology 2014; 5: 39.

10. San-Juan OD, Chiappa KH, Costello DJ, Cole AJ. Periodic epileptiform discharges in hypoxic encephalopathy: BiPLEDs and GPEDs as a poor prognosis for survival. Seizure 2009; 18: 365-368.

11. Legriel S, Hilly-Ginoux J, Resche-Rigon M, Merceron S, Pinoteau J, Henry-Lagarrigue M, et al. Prognostic value of electrographic postanoxic status epilepticus in comatose cardiac-arrest survivors in the therapeutic hypothermia era. Resuscitation 2013; 84: 343-350.

12. Mani R, Schmitt SE, Mazer M, Putt ME, Gaieski DF. The frequency and timing of epileptiform activity on continuous electroencephalogram in comatose post-cardiac arrest syndrome patients treated with therapeutic hypothermia. Resuscitation 2012; 83: 840-847.

13. Brenner RP. Is It Status? Epilepsia 2002; 43: 103-113.

14. Chong DJ, Hirsch LJ. Which EEG Patterns Warrant Treatment in the Critically Ill? Reviewing the Evidence for Treatment of Periodic Epileptiform Discharges and Related Patterns. Journal of Clinical Neurophysiology 2005; 22: 79-91.

15. Hirsch LJ, LaRoche SM, Gaspard N, Gerard E, Svoronos A, Herman ST, et al. American Clinical Neurophysiology Society's Standardized Critical Care EEG Terminology. Journal of Clinical Neurophysiology 2013; 30: 1-27. 
16. Tjepkema-Cloostermans MC, Hindriks R, Hofmeijer J, van Putten MJAM. Generalized periodic discharges after acute cerebral ischemia: Reflection of selective synaptic failure? Clinical Neurophysiology 2014; 125: 255-262.

17. Young GB, Gilbert JJ, Zochodne DW. The significance of myoclonic status epilepticus in postanoxic coma. Neurology 1990; 40: 1843-1848.

18. Bouwes A, Van Rootselaar AF, Biemond-Moeniralam HS, Teunissen LL, Tromp SC, Hijdra A, et al. Status epilepticus after cardiopulmonary resuscitation: a case cohort study. In: Postanoxic coma: prognosis after therapeutic hypothermia (PhD thesis); 2012, p. 117-129.

19. Abend NS, Dlugos DJ, Hahn CD, Hirsch LJ, Herman ST. Use of EEG monitoring and management of non-convulsive seizures in critically ill patients: a survey of neurologists. Neurocritical Care 2010; 12: 382-389.

20. Kilbride RD, Costello DJ, Chiappa KH. How seizure detection by continuous electroencephalographic monitoring affects the prescribing of antiepileptic medications. Archives of neurology 2009; 66: 723-728.

21. Bouwes A, Kuiper MA, Hijdra A, Horn J. Induced hypothermia and determination of neurological outcome after CPR in ICUs in the Netherlands: results of a survey. Resuscitation 2010; 81: 393-397.

22. Fujikawa DG. Prolonged seizures and cellular injury: understanding the connection. Epilepsy \& Behavior 2005; 7: S3-S11.

23. Naylor DE. Trafficking of GABAA Receptors, Loss of Inhibition, and a Mechanism for Pharmacoresistance in Status Epilepticus. Journal of Neuroscience 2005; 25: 7724-7733.

24. Zandbergen EGJ, Van Dijk JG, Hoedemaekers C, Horn J, Hijdra A, Kuiper MA, et al.. Richtlijn prognose van post-anoxisch coma [guideline]. Nederlandse Vereniging voor Neurologie, Nederlandse Vereniging voor Intensive Care; 2011. Available from: http://www.neurologie. nl/files/2713/1408/9483/RichtlijnPostanoxischcoma.pdf.

25. Brophy GM, Bell R, Claassen J, Alldredge B, Bleck TP, Glauser T, et al. Guidelines for the evaluation and management of status epilepticus. Neurocritical care 2012; 17: 3-23.

26. Rossetti AO, Lowenstein DH. Management of refractory status epilepticus in adults: still more questions than answers. Lancet neurology 2011; 10: 922-930.

27. Ware JE, Sherbourne CD. The MOS 36-item short-form health survey (SF-36). I. Conceptual framework and item selection. Medical Care 1992; 30: 473-483.

28. Montgomery SA, Asberg M. A new depression scale designed to be sensitive to change. British Journal of Psychiatry 1979; 134: 382-389.

29. Celesia GG, Grigg MM, Ross E. Generalized status myoclonicus in acute anoxic and toxicmetabolic encephalopathies. Archives of Neurology 1988; 45: 781-784.

30. Hui ACF, Cheng C, Lam A, Mok V, Joynt GM. Prognosis following Postanoxic Myoclonus Status epilepticus. European Neurology 2005; 54: 10-13.

31. Krumholz A, Stem BJ, Weiss HD. Outcome from coma after cardiopulmonary resuscitation: Relation to seizures and myoclonus. Neurology 1988; 38: 401-405. 


\section{A The TELSTAR study group}

\section{Executive Committee}

J. Hofmeijer (principal investigator)

B.J. Ruijter (coordinator)

M.J.A.M. van Putten

J. Horn

M.J. Blans

A. Beishuizen

A.F. van Rootselaar

\section{Steering Committee}

W.M. van den Bergh

J.W.J. Elting

S.C. Tromp

E. Scholten

N.A. Foudraine

F.H.M. Kornips

E.C. Thomeer

A. Dijkstra

F.S. Taccone

N. Gaspard

V.H.J.M. van Kranen-Mastenbroek

W.N.K.A. van Mook

C.W.E. Hoedemaekers

F.A.P. Nijhuis

J.A.M. van der Palen

(clinical epidemiologist)

\section{Data Safety Monitoring Board}

H.B. van der Worp (neurologist, chair)

A.J.C. Slooter (neurologist-intensivist)

M. van Smeden (biostatistician)
University of Twente, Rijnstate Hospital

University of Twente

University of Twente, Medisch Spectrum Twente

Academic Medical Center

Rijnstate Hospital

Medisch Spectrum Twente

Academic Medical Center

University Medical Center Groningen

University Medical Center Groningen

St Antonius Hospital

St Antonius Hospital

VieCuri Medical Center

VieCuri Medical Center

Maasstad Hospital

Maasstad Hospital

Hôpital Erasme (Brussels)

Hôpital Erasme (Brussels)

Maastricht UMC+

Maastricht UMC+

Radboudumc

Canisius-Wilhelmina Hospital

University of Twente
University Medical Center Utrecht University Medical Center Utrecht Leiden University Medical Center

\section{Independent expert}

K.H. Kho (neurosurgeon)

Medisch Spectrum Twente 


\section{B Trial status}

\section{Patient enrollment}

Recruitment to TELSTAR started in May 2014, with five participating hospitals. The number of centers has grown to eleven by now. Currently, 113 patients have been included, with the last inclusion expected by the end of 2019. Figure 7.B.1 provides more details on the study progress.
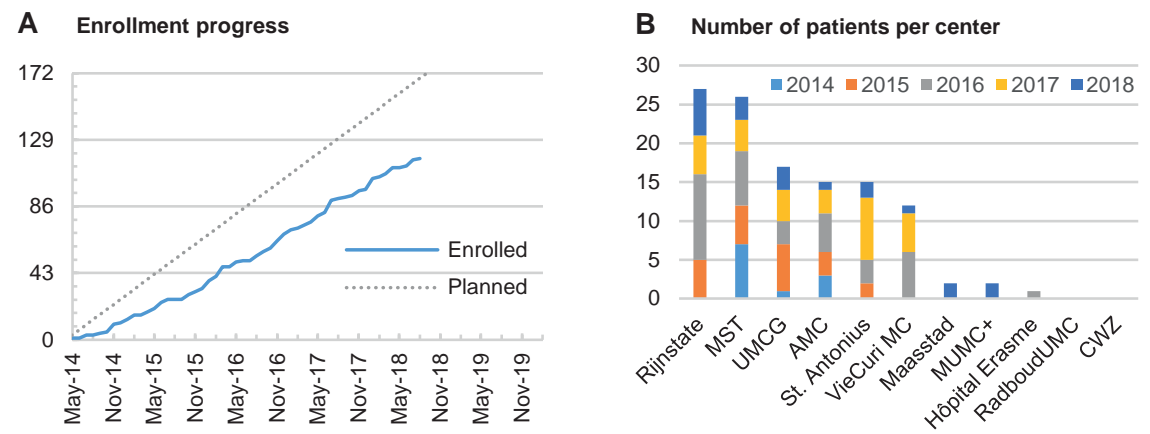

Figure 7.B.1: Overview of trial enrollment progress. This figure was last updated on August 14, 2018.

\section{Interim analyses}

The independent Data Safety Monitoring Board (DSMB) reviews data after every 43 subjects ( $25 \%$ of total) have had their primary outcome measurement. Up to now, two reviews have taken place:

- August 2016 (43 patients): analysis of safety

- November 2017 (86 patients): analysis of safety and efficacy

The DSMB advised both times to continue the trial as planned. Their last review will take place when the primary outcome of the 129th subject is known, and focuses on safety aspects. 



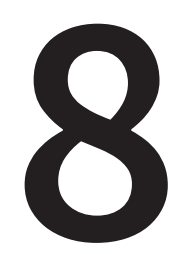

General discussion 
The research in this dissertation has established the value of continuous EEG for outcome prediction of comatose patients after cardiac arrest. This includes visual and quantitative methods in large prospective cohort studies, and quantification of the effects of sedative medication. The value for decisions on treatment with antiepileptic drugs is tested with the ongoing multicenter TELSTAR trial. Additionally, with a biophysical model, we identified candidate mechanisms that underlie specific pathological EEG patterns and their evolution, opening avenues for targeted treatments.

\subsection{Early EEG for outcome prediction after cardiac arrest}

Immediately after cardiac arrest, most patients have isoelectric or discontinuous EEG traces ${ }^{1}$ due to a massive synaptic arrest ${ }^{2}$. Research conducted before the introduction of TTM showed that a good outcome is possible if some cortical activity returns within 10 minutes to 8 hours $^{3}$, and only when continuous activity returns within 24 hours after cardiac arrest ${ }^{1}$.

The introduction of TTM has blurred these findings, but still the first 24 hours are the most valuable for discrimination between patients with or without chance of recovery $^{4-6}$. Therefore, timing of the EEG in relation to cardiac arrest is essential in interpretation ${ }^{7}$. EEG categories based on criteria for amplitude and background continuity have proven to be the most powerful predictors of outcome. The introduction of standardized terminology for EEG patterns in intensive care patients has been an important step to improve reproducibility of research findings ${ }^{8}$. In this dissertation, we integrated all these aspects in a large prospective cohort study.

\subsubsection{Prediction of poor outcome}

In chapter 2, we show that generalized suppression (all activity $<10 \mu \mathrm{V}$ ) or synchronous patterns with $\geq 50 \%$ suppression predict a poor outcome without false positives at $12 \mathrm{~h}$ after cardiac arrest or later. The latter group included generalized periodic discharges on a suppressed $(<10 \mu \mathrm{V})$ background and burst-suppression patterns with generalized bursting, abrupt burst onsets, and at least $50 \%$ suppression. At $12 \mathrm{~h}$ after cardiac arrest, the summed sensitivity of these patterns was $47 \%$, at $100 \%$ specificity.

As compared to other determinants of poor outcome after cardiac arrest, the sensitivity of an unfavorable EEG pattern is good. It is comparable to that for bilaterally absent SSEP responses after rewarming (36-48\%), and better than the combination of absent pupillary light responses and absent corneal reflexes (7-26\%) ${ }^{9}$. SSEP and EEG are complementary, since they identify different patients with poor outcome ${ }^{4,10}$. An advantage of EEG over other methods is its preserved reliability during hypothermia and sedation, which allows for earlier prognostication.

The poor prognosis of persisting suppression confirms previous observations that a failure of timely restoration of cortical activity impedes recovery. The previously reported low-voltage criterion (all activity $<20 \mu \mathrm{V}$ ) at $24 \mathrm{~h}$ or later was not $100 \%$ specific for the prediction of poor outcome, since two patients with a low voltage patterns at 36 h eventually recovered ${ }^{4,5}$. The prognostic value of generalized suppression cannot be generalized to time points earlier than $6 \mathrm{~h}$ after cardiac arrest, since in the first minutes to hours after cardiac arrest, most patients will have a suppressed EEG. This does 
not exclude chance of recovery ${ }^{3,11}$.

The group of 'synchronous patterns with $\geq 50 \%$ suppression' can be considered as generalization of the previously reported group of 'burst-suppression patterns with identical bursts' ${ }^{12}$. By broadening the definition, we substantially increased the sensitivity for the prediction of poor outcome as compared to earlier work, without loss of specificity. Previous studies disagreed on the prognostic value of burst-suppression patterns ${ }^{4-6}$. We show that this probably results from inconsistent definitions and the fact that only a subgroup of burst-suppression, with highly synchronized burst types, is a reliable predictor of poor outcome.

\subsubsection{Prediction of good outcome}

Early information on chance of recovery can be reassuring for relatives and could help treating physicians when making decisions on intensive treatment of accompanying medical problems. Still, most prognostication studies in postanoxic coma put emphasis on predictions of poor outcome. EEG is one of the few, if not the only, highly specific method that can be used to predict good outcome during sedation and hypothermia after cardiac arrest ${ }^{4,13}$.

In chapter 2, we confirm that restoration of a continuous, normal amplitude background, without periodic activity, within $12 \mathrm{~h}$ after cardiac arrest is a specific predictor of good outcome. Sensitivity was 50\%, at $91 \%$ specificity. Of those $9 \%$ that did not have a good outcome, most died at the ICU as a result of failure of other organs than the brain, often a second cardiac arrest. In chapter 5, we showed that propofol in the typical doses used reduces background continuity with $10-20 \%$, indicating that small discontinuities do not exclude chance of recovery. This justifies our choice to consider nearly continuous EEG patterns ( $<10 \%$ suppression) as favorable and supports the notion that propofol does not impede predictive values for poor outcome prediction.

\subsubsection{Additional value of reactivity testing}

In this dissertation, we only analyzed spontaneous EEG patterns and did no additional reactivity testing. Although a single group claims high specificity of absent EEG background reactivity for prediction of poor outcome ${ }^{13}$, others find high numbers of false positives ${ }^{5,14,15}$. Interobserver agreement for reactivity testing is low ${ }^{16}$. Most likely, this results from a lack of standardization of stimulus types, timing, and quantitative measurement of responses ${ }^{17}$. Future work should assess the added value of standardized reactivity testing for prognostication in postanoxic coma.

\subsubsection{Continuous versus intermittent EEG recordings}

An important question is whether continuous monitoring after cardiac arrest is of added value as compared to a routine 20-minute EEG recording. In chapter 2, we show that repeated evaluation of the EEG within the first 24 hours after cardiac arrest increased the number of patients with reliable outcome predictions, as compared to a single evaluation. The added value of evaluation beyond $24 \mathrm{~h}$ was limited. Our findings contradict a previous study which claimed that one routine EEG recording during hypothermia, plus one at normothermia and off-sedation, had equal value for outcome prediction as continuous EEG recordings ${ }^{18}$. The authors of that study 
seemed to neglect the fact that most evolutional changes of the postanoxic EEG take place within the first 24 hours after cardiac arrest.

We show in chapter 2 that significantly more patients with good or poor outcomes can be identified if continuous EEG recordings are started within 6-12 hours after cardiac arrest, as compared to later starting times. If centers do not have the facilities to do continuous EEG monitoring after cardiac arrest, the optimal timing of a single routine recording for prognostication would be between 12 and $24 \mathrm{~h}$ after cardiac arrest.

\subsection{Value of quantitative EEG analysis after cardiac arrest}

Despite extensive training and the use of standardized of terminology, EEG interpretation remains subjective to some extent, leading to interrater variability. Additionally, reviewing long-term EEG-recordings on the ICU is time-consuming, and in most cases it will not be possible to have every single page of the recording reviewed by a technician or physician. Quantitative EEG may help to overcome these issues and can be applied by non-experts. Furthermore, quantitative EEG analyses may improve outcome predictions by increasing the amount of relevant information that can be extracted. Various quantitative EEG features have been proposed for assistance in outcome predictions after cardiac arrest, as single features or combined into an index ${ }^{19}$.

Quantitative EEG methods can be subdivided into two types. In one of them, quantitative EEG detects information that is hard or impossible to detect by a human observer. This includes measures of entropy and information quantity, amongst others ${ }^{19}$. Such methods have the advantage of adding new information to what the electroencephalographer can see. On the other hand, such measures cannot easily be traced back to characteristics observed during visual analysis, which may limit clinical acceptance. A second approach is to use quantitative EEG as a 'surrogate encephalographer'. By doing so, quantitative features capture key characteristics that an experienced human reviewer would use during visual assessment. Examples include measures for amplitude, frequency content and background continuity. Such methods are intuitively clear and can capture features that appear visually clear, but hard to quantify by a human observer. These can be extrapolated to the clinic, where visual assessment remains the gold standard, relatively easy.

In this dissertation, we used the 'surrogate encephalographer' approach. In chapter 3, we reduced a number of discriminative features from visual analysis into only two novel quantitative EEG features: the background continuity index and the burst-suppression amplitude ratio. From this combination, we obtained sensitivities and specificities for the prediction of poor and good outcome equal to those of visual assessment of EEG. To the best of our knowledge, no other single qEEG feature reported better prediction of outcome than these measures. Within its confidence intervals, predictive values were the same as for the revised Cerebral Recovery Index, a combined index using nine quantitative EEG features that has the highest reported values for the outcome prediction of postanoxic coma, so far ${ }^{20}$. Apparently, background continuity and the degree of amplitude fluctuation comprise an important part of the predictive information included in the postanoxic EEG. 


\subsection{Sedation, hypothermia, and reliability of EEG analysis}

The studies in this dissertation confirm that the postanoxic EEG includes most discriminative information within the first 24 hours after cardiac arrest. With current treatment standards, this coincides with the period of targeted temperature management and sedation. Our work indicates that the effect of propofol on the EEG is small, as compared to the effects of postanoxic brain injury.

Propofol can induce discontinuities in the EEG and, at higher doses, even electrocerebral silence ${ }^{25}$. Midazolam reduces the EEG amplitude ${ }^{26}$ and may as well induce burstsuppression patterns in critically ill patients ${ }^{27}$. Since amplitude and background continuity of the EEG are included in all key outcome predictors of comatose patients after cardiac arrest, there is a relevant risk of influence by sedative medication. We show in chapter 5 that propofol reduces the amplitude and continuity of postanoxic EEG, indeed, but with absolute effects sizes that are much smaller than those of hypoxia. These results make it very unlikely that propofol, in the doses applied, induces the typically observed pathological burst-suppression of flat EEG patterns.

There are a few situations in which one should be cautious when assessing the EEG under sedation. For propofol doses above $6 \mathrm{mg} / \mathrm{kg} / \mathrm{h}$, or when high dosesa combination of midazolam and propofol are used in combination is used, we cannot exclude that sedation increases the chance of falsely pessimistic predictions of outcome.

Studies on the effect of hypothermia on the EEG show that, for temperatures between 32 and $36^{\circ} \mathrm{C}$, background continuity remains preserved and only small frequency shifts are induced ${ }^{21,22}$. This issue will probably become less important in the future, since more and more centers apply normothermia after the recent TTM trial ${ }^{23,24}$.

\subsection{Self-fulfilling prophecy}

An important limitation of most, if not all, studies on prognostication of neurological outcome after cardiac arrest is the risk of a self-fulfilling prophecy. This is the bias that occurs when physicians use the outcome measure under study in decisions on withdrawal of life-sustaining treatment ${ }^{28}$. To avoid such a bias, we conducted analysis of EEG-related measures offline, after treatment of each patient was finished. Decisions on treatment discontinuation were based on international guidelines that use neurological examination and absent SSEP responses, and the EEG within the first 72 hours was not taken into account ${ }^{29,30}$. However, to allow for detection and treatment of electrographic seizure activity, treating physicians were not blinded to EEG recordings. In the ideal study situation, one should define a minimum treatment duration for all patients, but this seems undesirable in the setting of regular patient care in the Netherlands.

\subsection{Mechanisms that drive postanoxic EEG patterns}

Although there is ample literature about microscopic mechanisms of hypoxic-ischemic brain injury, the dynamics that drive postanoxic EEG patterns are poorly understood. Research on the value of the EEG after cardiac arrest is dominated by phenomenological work, in which EEG patterns are related to a chance of recovery. Understanding 
the link between EEG pattern and underlying process could help to improve targeted treatment strategies, where therapy could be guided and monitored by EEG patterns. Computational modeling and simulation can help to bridge this gap of knowledge.

In this dissertation, we investigated the effect of hypoxia in a computational mean field model of the cerebral cortex, comprising inhibitory and excitatory neuronal populations, their synaptic interactions and thalamic input. We found that an increased synaptic excitation-inhibition ratio, caused by anoxic long term potentiation, explained common patterns observed. A gradual recovery of synaptic metabolism, resulting in an improved recovery rate of synapses, explained typical transitions as observed in patients.

Possible future improvements of the model include the addition of spatial heterogeneities and cortico-thalamic interactions ${ }^{31}$. This will likely improve the understanding of spatially heterogeneous patterns and the propagation of EEG waves through the cortex. It may also provide explanations why spatially synchronized patterns have such a strong correlation with poor outcome, whereas spatially heterogeneous patterns usually indicate a more favorable outcome.

One of the ultimate goals of clinically motivated computational models is to find potentially effective treatment targets. We observed in our model that propofol sedation could reverse pathological EEG patterns, at least temporarily. However, to prevent massive secondary neural cell death, the increased synaptic excitation-inhibition must be reversed permanently. A potential compensatory mechanism would be homeostatic synaptic scaling ${ }^{32}$. Since this process takes place on time scales of days, this would require prolonged treatment with drugs that antagonize glutamatergic action, such as propofol.

\subsection{Treatment of electrographic status epilepticus}

Electrographic activity with epileptiform morphology is commonly observed in patients after cardiac arrest ${ }^{33-35}$. Most often it consists of generalized periodic discharges. Electrographic seizures, with typical waxing and waning of epileptiform discharges, are uncommon ${ }^{36}$. In comatose patients after cardiac arrest, epileptiform activity differs from that in most patients with epilepsy, who have relatively abrupt seizure onset and often a temporal and spatial evolution of the discharges. In postanoxic encephalopathy, patterns with epileptiform morphology typically appear gradually, over a time course of hours to days, in which the onset time is to some extent arbitrary. Additionally, brain functioning is severely impaired before the onset of this activity, and in most cases consciousness does not return, despite treatment. In some cases, epileptiform patterns in postanoxic coma are accompanied by clinical phenomena, such as myoclonus.

Whether treatment with anti-epileptic drugs or sedation improves outcome of patients with electrographic seizure-like activity in postanoxic coma remains an open question. Animal research indicates that prolonged seizure activity induces brain injury, no matter whether this activity is convulsive or non-convulsive ${ }^{37}$. This advocates the use of antiepileptic treatment, in order to prevent secondary neuronal injury. Our computational model, as discussed in chapter 6 , suggests that treatment should counterbalance 
the increase in NMDA-receptor gated currents. Additionally, the model indicates that burst-suppression and GPDs should be treated equally, since they result from a common mechanism. A quantitative EEG study, as described in chapter 4, suggests that restoration of continuous background activity before the onset of status epilepticus is essential for a chance of recovery.

In chapter 7, we present an ongoing, multicenter, randomized controlled trial on aggressive treatment of electrographic status epilepticus in comatose patients after cardiac arrest. Although the expected chance of survival in this group of patients is low, we hope to identify subgroups of EEG patterns in which treatment is beneficial. The trial started in 2014. Inclusion is expected to be complete in the second half of 2019.

\subsection{Future perspectives}

This dissertation provides high quality evidence for early EEG, recorded within the first 24 hours after cardiac arrest, to be a reliable predictor of outcome of comatose patients after cardiac arrest. Our results advocate the introduction of early EEG in guidelines on outcome prediction and may influence decisions on withdrawal of life-sustaining treatment.

The use of computer assistance in EEG analysis will become more important in the near future. Its predictive accuracy in postanoxic coma could be further improved by capturing information about the evolution of EEG patterns, by integrating information from consecutive time points. Additionally, the combined value of quantitative and visual analysis should be investigated. An upcoming branch of machine learning is deep learning ${ }^{38}$, in which the computer learns from experience, without the need of a human computer operator to specify any knowledge. Our recent work shows promising results on the application of this technique on EEG data of comatose patients after cardiac arrest $^{39}$. We expect that future developments will allow reliable predictions of outcome with EEG in up to $90 \%$ of these patients.

The TELSTAR trial (www.telstartrial.nl) on treatment of status epilepticus after cardiac arrest is ongoing. Any outcome of this trial will change international guidelines, since both aggressive treatment and no medical treatment are currently considered standard modalities. If treatment is shown to be futile, the trial will prevent unnecessary prolongation of intensive care treatment and shorten periods of uncertainty for relatives of future patients. If treatment is effective, it will be included in guidelines to promote functional recovery of comatose patients after cardiac arrest.

Most studies on neuroprotective drugs after cardiac arrest have focused on inhibition of neuronal activity, and none of them has been proven to improve outcome ${ }^{40}$. An alternative treatment approach would be to use mild pharmacological stimulation, which was shown to improve recovery of cultured neurons exposed to hypoxia ${ }^{41}$. Mild neuronal stimulation with ghrelin will be tested in a multicenter randomized trial that is expected to start in September 2019. Further improvements of our understanding of the pathophysiology of brain damage after cardiac arrest and the consequent EEG rhythms may be derived from computational models. At best, these insights will contribute to future EEG-guided targeted treatments. 


\subsection{Conclusions}

Early EEG recordings in comatose survivors of cardiac arrest allow for reliable prediction of good and poor outcome. Normal amplitude, continuous background patterns without periodic activity are specific for good outcome at 6 and $12 \mathrm{~h}$ after cardiac arrest. Generalized suppression and synchronous patterns with more than $50 \%$ suppression at $12 \mathrm{~h}$ or later are reliable predictors of poor outcome. The added prognostic value of continuous EEG monitoring beyond $24 \mathrm{~h}$ after cardiac arrest is limited.

Simple quantitative measures of background continuity and the amplitude ratio between bursts and suppressions allow for outcome predictions that are equally reliable as visual analysis. A lack of background continuity at $24 \mathrm{~h}$ after cardiac arrest and a bursts-suppression amplitude ratio $>6.2$ predict poor outcome with $100 \%$ specificity. In patients with electrographic status epilepticus, lack of background continuity precludes recovery.

Propofol sedation decreases the amplitude and background continuity of the postanoxic EEG, but these effects are too small to influence the reliability of predictions of outcome.

Pathophysiological mechanisms at the synaptic level explain the most commonly observed EEG patterns after cardiac arrest and their evolution.

Whether treatment of electrographic status epilepticus improves the outcome of comatose patients after cardiac arrest is currently under investigation in the ongoing multicenter TELSTAR trial.

\section{References}

1. Jørgensen E, Holm S. The natural course of neurological recovery following cardiopulmonary resuscitation. Resuscitation 1998; 36: 111-122.

2. Hofmeijer J, van Putten MJAM. Ischemic cerebral damage: an appraisal of synaptic failure. Stroke 2012; 43: 607-615.

3. Jørgensen E, Malchow-Møller A. Natural history of global and critical brain ischaemia. Part I: EEG and neurological signs during the first year after cardiopulmonary resuscitation in patients subsequently regaining consciousness. Resuscitation 1981; 9: 133-153.

4. Hofmeijer J, Beernink TMJ, Bosch FH, Beishuizen A, Tjepkema-Cloostermans MC, Van Putten MJAM. Early EEG contributes to multimodal outcome prediction of postanoxic coma. Neurology 2015; 85: 137-143.

5. Sivaraju A, Gilmore EJ, Wira CR, Stevens A, Rampal N, Moeller JJ, et al. Prognostication of post-cardiac arrest coma: early clinical and electroencephalographic predictors of outcome. Intensive Care Medicine 2015; 41: 1264-1272.

6. Spalletti M, Carrai R, Scarpino M, Cossu C, Ammannati A, Ciapetti M, et al. Single electroencephalographic patterns as specific and time-dependent indicators of good and poor outcome after cardiac arrest. Clinical Neurophysiology 2016; 127: 2610-2617.

7. Hofmeijer J, Van Putten MJAM. EEG in postanoxic coma: Prognostic and diagnostic value. Clinical Neurophysiology 2016; 127: 2047-2055.

8. Hirsch LJ, LaRoche SM, Gaspard N, Gerard E, Svoronos A, Herman ST, et al. American Clinical Neurophysiology Society's Standardized Critical Care EEG Terminology. Journal of Clinical Neurophysiology 2013; 30: 1-27.

9. Sandroni C, Cavallaro F, Callaway CW, D'Arrigo S, Sanna T, Kuiper Ma, et al. Predictors of poor neurological outcome in adult comatose survivors of cardiac arrest: a systematic review and 
meta-analysis. Part 2: Patients treated with therapeutic hypothermia. Resuscitation 2013; 84: 1324-1338.

10. van Putten MJ. The N20 in post-anoxic coma: Are you listening? Clinical Neurophysiology 2012; 123: 1460-1464.

11. Lamartine Monteiro M, Taccone FS, Depondt C, Lamanna I, Gaspard N, Ligot N, et al. The Prognostic Value of 48-h Continuous EEG During Therapeutic Hypothermia After Cardiac Arrest. Neurocritical Care 2016; 24: 153-162.

12. Hofmeijer J, Tjepkema-Cloostermans MC, van Putten MJAM. Burst-suppression with identical bursts: A distinct EEG pattern with poor outcome in postanoxic coma. Clinical Neurophysiology 2014; 125: 947-954.

13. Rossetti AO, Tovar Quiroga DF, Juan E, Novy J, White RD, Ben-Hamouda N, et al. Electroencephalography Predicts Poor and Good Outcomes After Cardiac Arrest. Critical Care Medicine 2017; 45: e674-e682.

14. Amorim E, Rittenberger JC, Zheng JJ, Westover MB, Baldwin ME, Callaway CW, et al. Continuous EEG monitoring enhances multimodal outcome prediction in hypoxic-ischemic brain injury. Resuscitation 2016; 109: 121-126.

15. Grippo A, Carrai R, Scarpino M, Spalletti M, Lanzo G, Cossu C, et al. Neurophysiological prediction of neurological good and poor outcome in post-anoxic coma. Acta Neurologica Scandinavica 2017; 135: 641-648.

16. Westhall E, Rosén I, Rossetti AO, van Rootselaar AF, Wesenberg Kjaer T, Friberg H, et al. Interrater variability of EEG interpretation in comatose cardiac arrest patients. Clinical Neurophysiology 2015; 126: 2397-2404.

17. Admiraal MM, van Rootselaar AF, Horn J. Electroencephalographic reactivity testing in unconscious patients: a systematic review of methods and definitions. European Journal of Neurology 2017; 24: 245-254.

18. Alvarez V, Sierra-Marcos A, Oddo M, Rossetti AO. Yield of intermittent versus continuous EEG in comatose survivors of cardiac arrest treated with hypothermia. Critical Care 2013; 17: R190.

19. Asgari S, Moshirvaziri H, Scalzo F, Ramezan-Arab N. Quantitative measures of EEG for prediction of outcome in cardiac arrest subjects treated with hypothermia: a literature review. Journal of Clinical Monitoring and Computing 2018; p. 1-16.

20. Tjepkema-Cloostermans MC, Hofmeijer J, Beishuizen A, Hom HW, Blans MJ, Bosch FH, et al. Cerebral Recovery Index: Reliable help for prediction of neurologic outcome after cardiac arrest. Critical Care Medicine 2017; 45: 789-797.

21. Kochs E. Electrophysiological monitoring and mild hypothermia. Journal of Neurosurgical Anesthesiology 1995; 7: 222-228.

22. Stecker MM, Cheung AT, Pochettino A, Kent GP, Patterson T, Weiss SJ, et al. Deep hypothermic circulatory arrest: II. Changes in electroencephalogram and evoked potentials during rewarming. The Annals of thoracic surgery 2001; 71: 22-28.

23. Nielsen N, Wetterslev J, Cronberg T, Erlinge D, Gasche Y, Hassager C, et al. Targeted temperature management at $33 \mathrm{C}$ versus $36 \mathrm{C}$ after cardiac arrest. The New England Journal of Medicine 2013; 369: 2197-2206.

24. Storm C, Nee J, Sunde K, Holzer M, Hubner P, Taccone FS, et al. A survey on general and temperature management of post cardiac arrest patients in large teaching and university hospitals in 14 European countries-The SPAME trial results. Resuscitation 2017; 116: 8490.

25. San-Juan D, Chiappa KH, Cole AJ. Propofol and the electroencephalogram. Clinical Neurophysiology 2010; 121: 998-1006.

26. Veselis RA, Reinsel R, Marino P, Sommer S, Carlon GC. The effects of midazolam on the EEG during sedation of critically ill patients. Anaesthesia 1993; 48: 463-470. 
27. ter Horst $\mathrm{H}$, Brouwer $\mathrm{O}, \mathrm{Bos} \mathrm{A}$. Burst suppression on amplitude-integrated electroencephalogram may be induced by midazolam: a report on three cases. Acta Paediatrica 2004; 93 : 559-563.

28. Geocadin RG, Peberdy MA, Lazar RM. Poor survival after cardiac arrest resuscitation. Critical Care Medicine 2012; 40: 979-980.

29. Sandroni C, Cariou A, Cavallaro F, Cronberg T, Friberg H, Hoedemaekers C, et al. Prognostication in comatose survivors of cardiac arrest: An advisory statement from the European Resuscitation Council and the European Society of Intensive Care Medicine. Resuscitation 2014; 85: 1779-1789.

30. Wijdicks EFM, Hijdra A, Young GB, Bassetti CL, Wiebe S. Practice parameter: prediction of outcome in comatose survivors after cardiopulmonary resuscitation (an evidence-based review): report of the Quality Standards Subcommittee of the American Academy of Neurology. Neurology 2006; 67: 203-210.

31. Rennie CJ, Robinson Pa, Wright JJ. Unified neurophysical model of EEG spectra and evoked potentials. Biological cybernetics 2002; 86: 457-471.

32. Turrigiano GG. The Self-Tuning Neuron: Synaptic Scaling of Excitatory Synapses. Cell 2008; 135: 422-435.

33. Legriel S, Bruneel F, Sediri H, Hilly J, Abbosh N, Lagarrigue MH, et al. Early EEG monitoring for detecting postanoxic status epilepticus during therapeutic hypothermia: a pilot study. Neurocritical Care 2009; 11: 338-344.

34. Rittenberger JC, Popescu A, Brenner RP, Guyette FX, Callaway CW. Frequency and timing of nonconvulsive status epilepticus in comatose post-cardiac arrest subjects treated with hypothermia. Neurocritical Care 2012; 16: 114-122.

35. Rossetti AO, Oddo M, Liaudet L, Kaplan PW. Predictors of awakening from postanoxic status epilepticus after therapeutic hypothermia. Neurology 2009; 72: 744-749.

36. Cloostermans MC, van Meulen FB, Eertman CJ, Hom HW, van Putten MJAM. Continuous electroencephalography monitoring for early prediction of neurological outcome in postanoxic patients after cardiac arrest: A prospective cohort study. Critical Care Medicine 2012; 40: 2867-2875.

37. Fujikawa DG. Prolonged seizures and cellular injury: understanding the connection. Epilepsy \& Behavior 2005; 7: S3-S11.

38. LeCun YA, Bengio Y, Hinton GE. Deep learning. Nature 2015; 521: 436-444.

39. van Putten MJAM, Hofmeijer J, Ruijter BJ, Tjepkema-Cloostermans MC. Deep Learning for outcome prediction of postanoxic coma. In: IFMBE Proceedings. vol. 65; 2018, p. 506-509.

40. Wright W, Geocadin R. Postresuscitative Intensive Care: Neuroprotective Strategies after Cardiac Arrest. Seminars in Neurology 2006; 26: 396-402.

41. Stoyanova II, Hofmeijer J, van Putten MJAM, le Feber J. Acyl Ghrelin Improves Synapse Recovery in an In Vitro Model of Postanoxic Encephalopathy. Molecular Neurobiology 2016; 53 : 6136-6143. 


\section{Summary}

After a successful resuscitation from cardiac arrest, most patients remain comatose as a result of postanoxic encephalopathy. More than half of them will never regain consciousness, and treatment options to improve outcome are limited. The aim of the research described in this dissertation is to validate and improve the value of continuous electroencephalography (EEG) for outcome prediction and treatment of postanoxic brain injury.

In chapter 2, we confirm that early EEG is a sensitive and reliable tool for the prediction of outcome. In a five-center prospective cohort study including 850 patients, we showed that the predictive value of EEG reaches its peak within the first 24h after cardiac arrest. At $12 \mathrm{~h}$ after cardiac arrest, favorable EEG patterns (continuous or nearly continuous background, amplitude $\geq 20 \mu \mathrm{V}$, no periodic activity) predicted good outcome (Cerebral Performance Category (CPC 1 or 2) with sensitivity of 0.50 (95\% confidence interval (Cl): $0.46-0.55)$ and specificity of 0.91 (95\%-Cl: $0.88-0.93$ ). For later time points, sensitivity for good outcome increased even further, but at the cost of specificity. Unfavorable EEG patterns (generalized suppression (all activity $<10 \mu \mathrm{V}$ ) or synchronous patterns with at least $50 \%$ suppression) invariably predicted poor outcome between $12 \mathrm{~h}$ and 5 days after cardiac arrest. At $12 \mathrm{~h}$, sensitivity for poor outcome was 0.47 (95\%-Cl: 0.42-0.51), and specificity was 1.00 (95\%-Cl: 0.99-1.00). At later time points, sensitivity for poor outcome decreased gradually. Predictions of outcome were equally reliable in centers with different treatment regimes.

In chapters 3 and 4 , we introduce straightforward quantitative EEG features, based on key aspects of visual assessment, with value for the outcome prediction of postanoxic coma. In chapter 3, we focus on characteristics of the EEG background pattern. The background continuity index $(\mathrm{BCl})$ was defined as the fraction of time not spend in suppressions (all activity $<10 \mu \mathrm{V}$ for $\geq 0.5 \mathrm{~s}$ ), and the burst-suppression amplitude ratio (BSAR) as the mean amplitude ratio between non-suppressed and suppressed EEG segments. At 24h after cardiac arrest, a combination of $\mathrm{BCl}$ and BSAR predicted good outcome with sensitivity of 0.57 (95\%-Cl: 0.48-0.67) and specificity of 0.90 (95\%-Cl: 0.86 $0.95)$. A BCl $<0.014$ or a BSAR $\geq 6.12$ predicted a poor outcome without false positives at $10 \mathrm{~h}$ after cardiac arrest or later. At $12 \mathrm{~h}$, a combination of $\mathrm{BCl}$ and BSAR predicted poor outcome with sensitivity of 0.50 (95\%-Cl: $0.42-0.57)$ and specificity of 1.00 (95\%$\mathrm{Cl}$ : 0.99-1.00). In chapter 4, we concentrate on patients with epileptiform EEG patterns. We showed that a good outcome is only possible if the epileptiform activity evolves from a continuous background pattern. Additionally, a good outcome was associated with a higher discharge frequency, lower relative discharge amplitude, and a lower discharge periodicity.

In chapter 5, we quantify the effects of propofol on the postanoxic EEG and assess its influence on the reliability of outcome predictions. After the interruption of propofol, 
at a median of $41 \mathrm{~h}$ after cardiac arrest, the specificity of favorable EEG patterns for good outcome decreased, and the predictive value of unfavorable EEG patterns for poor outcome remained unchanged. Quantitatively, propofol was associated with decreases of the EEG amplitude, $\mathrm{BCl}$ and alpha-delta ratio, and an increase of the BSAR. Despite these effects, in a multivariate model, propofol did neither increase the chance of an unfavorable EEG pattern (adjusted odds ratio (aOR) 0.92 per increase of $2 \mathrm{mg} / \mathrm{kg} / \mathrm{h}$, 95\%-Cl: 0.81-1.11) nor decrease the chance of a favorable EEG pattern (aOR 0.98, 95\%$\mathrm{Cl}$ : 0.81-1.09) on visual assessment.

Mechanisms underlying EEG patterns specific for postanoxic encephalopathy are largely unknown. In chapter 6 , we present a biophysical model in which we incorporate two candidate mechanisms at the synaptic level. In this mean field model, anoxic damage was modeled as aggravated short-term synaptic depression, with gradual recovery over many hours. Additionally, excitatory neurotransmission was potentiated, scaling with the severity of postanoxic encephalopathy. The model simulations corresponded well with six common categories of EEG rhythms in postanoxic brain injury, including typical transitions in time. Plausible results were only obtained if excitatory synapses were more severely affected than inhibitory synapses by short-term synaptic depression. The application of propofol in the model normalized the EEG abnormalities. However, permanent reversal was only possible with an additional mechanism for long-lasting normalization of the synaptic excitation-inhibition ratio.

Finally, in chapter 7, we present the study protocol of the ongoing, multicenter, randomized controlled TELSTAR trial. In this study, 172 patients with electrographic status epilepticus after cardiac arrest are randomly assigned to either aggressive medical treatment or no medical treatment. Any outcome of this trial will change international guidelines, since both treatment strategies are currently considered standard modalities. The trial started in 2014, and inclusion is expected to be complete in the second half of 2019. 


\section{Samenvatting}

$\mathrm{Na}$ een geslaagde reanimatie blijven het merendeel van de patiënten comateus ten gevolge van postanoxische encefalopathie. Bij meer dan de helft herstelt het bewustzijn nooit en behandelopties om de uitkomst te verbeteren zijn beperkt. Het onderzoek in dit proefschrift heeft als doel om de waarde van continue elektro-encefalografie (EEG) bij de prognosebepaling en behandeling van postanoxisch coma te valideren en te verbeteren.

In hoofdstuk 2 bevestigen we dat het EEG een sensitief en betrouwbaar hulpmiddel is bij de uitkomstvoorspelling van comateuze patiënten na een hartstilstand. In een prospectief cohort van 850 patiënten, verzameld in vijf centra, tonen we aan dat de prognostische waarde het hoogst is binnen de eerste 24 uur na de hartstilstand. Op 12 uur na de hartstilstand voorspelde een gunstig EEG-patroon (continue of bijna continue achtergrondactiviteit met amplitudes $\geq 20 \mu \mathrm{V}$ en afwezige periodieke activiteit) een goede uitkomst (Cerebral Performance Category 1-2) met sensitiviteit van 0.50 (95\% betrouwbaarheidsinterval (BI): 0.46-0.55) en een specificiteit van 0.91 (95\%-BI: 0.880.93). Op latere tijdstippen nam de sensitiviteit toe, maar ten koste van de specificiteit. Een ongunstig EEG-patroon (gegeneraliseerde suppressie (alle activiteit $<10 \mu \mathrm{V}$ ) of synchrone patronen met ten minste 50\% suppressie) voorspelde betrouwbaar een slechte uitkomst (Cerebral Performance Category 3-5), op ieder tijdstip tussen 12 uur en 5 dagen na de hartstilstand. Op 12 uur was de sensitiviteit voor voorspelling van slechte uitkomst 0.47 (95\%-BI: 0.42-0.51), bij een specificiteit van 1.00 (95\%-BI: 0.99-1.00). Op latere tijdstippen daalde de sensitiviteit voor slechte uitkomst geleidelijk. De betrouwbaarheid van uitkomstvoorspellingen was gelijk voor alle centra, ondanks verschillen in behandeling.

In hoofdstukken 3 en $\mathbf{4}$ introduceren we eenvoudige kwantitatieve EEG-maten, gebaseerd op aspecten die worden gebruikt bij visuele beoordeling van het EEG voor de uitkomstvoorspelling van postanoxisch coma. In hoofdstuk 3 ligt de nadruk op kenmerken van het grondpatroon. We definieerden de background continuity index $(\mathrm{BCl})$ als fractie van het signaal dat niet werd geclassificeerd als 'suppressie' (alle activiteit $<10 \mu \mathrm{V}$ gedurende $\geq 0.5 \mathrm{~s}$ ), en de burst-suppression amplitude ratio (BSAR) als de gemiddelde amplitudeverhouding tussen 'bursts' en suppressies. Op 24 uur na de hartstilstand voorspelde een combinatie van $\mathrm{BCl}$ en $\mathrm{BSAR}$ een goede uitkomst met een sensitiviteit van 0.57 (95\%-BI: 0.48-0.67), bij een specificiteit van 0.90 (95\%-BI: 0.86-0.95). Een $\mathrm{BCl}<0.014$ of een $\mathrm{BSAR} \geq 6.12$, vastgesteld op 10 uur na de hartstilstand of later, voorspelde betrouwbaar een slechte uitkomst. Op 12 uur voorspelde een combinatie van BCl en BSAR een slechte uitkomst met een sensitiviteit van 0.50 (95\%-BI: 0.420.57), bij een specificiteit van 1.00 (95\%-BI: 0.99-1.00). In hoofdstuk 4 richten we ons op de subgroep van patiënten met epileptiforme EEG-patronen. We toonden aan dat een goede uitkomst slechts mogelijk was indien de epileptiforme activiteit ontstond 
na herstel van continuïteit van het grondpatroon. Een goede uitkomst was verder geassocieerd met een hogere ontladingsfrequentie, een lage relatieve amplitude en een minder sterke periodiciteit van ontladingen.

In hoofdstuk 5 kwantificeren we de effecten van propofol op het postanoxische EEG en analyseren we of deze de betrouwbaarheid van uitkomstvoorspellingen beïnvloeden. $\mathrm{Na}$ het staken van propofol, op een mediaan van 41 uur na de hartstilstand, nam de specificiteit van een gunstig EEG-patroon voor goede uitkomst af, terwijl de waarde van een ongunstig EEG-patroon voor de voorspelling van een slechte uitkomst gelijk bleef. Bij kwantitatieve EEG-analyse was propofol geassocieerd met een afname van amplitude, $\mathrm{BCI}$, en alfa-delta-ratio, en een toename van de BSAR. Met een multivariabel model werd vastgesteld dat, ondanks de effecten van propofol op kwantitatief EEG, propofol de kans op een ongunstig EEG-patroon bij visuele beoordeling niet verhoogt (geadjusteerde odds ratio (aOR) 0.92 per toename van $2 \mathrm{mg} / \mathrm{kg} / \mathrm{uur}, 95 \%-\mathrm{Bl}$ : 0.81-1.11) en de kans op een gunstig EEG-patroon niet verlaagt (aOR 0.98, 95\%-BI: 0.81-1.09).

De mechanismen die verantwoordelijk zijn voor de specifieke EEG-patronen waargenomen bij postanoxische encefalopathie zijn grotendeels onbekend. In hoofdstuk 6 presenteren we een biofysisch model waarin twee mogelijke mechanismen op het niveau van de synaps zijn opgenomen. In dit zogenaamde mean field model hebben we anoxische schade gemodelleerd als een versterkte korte-termijndepressie van synaptische activiteit, met een geleidelijk herstel over vele uren. Daarnaast werd exciterende neurotransmissie versterkt, in een mate die schaalde met de ernst van de postanoxische encefalopathie. De gesimuleerde EEG-patronen kwamen goed overeen met zes veelvoorkomende patronen bij postanoxisch coma, inclusief de kenmerkende overgangen tussen patronen die optreden wanneer tijd verstrijkt. Plausibele resultaten werden alleen verkregen indien de korte-termijndepressie van synaptische activiteit sterker was aangedaan bij exciterende synapsen dan bij inhiberende synapsen. De toevoeging van propofol in het model kon de gesimuleerde pathologische EEG-patronen laten verdwijnen. Echter, om deze patronen voorgoed te laten verdwijnen was het nodig om een mechanisme toe te voegen voor normalisatie van de synaptische excitatie-inhibitieratio op de lange termijn.

In hoofdstuk 7 presenteren we het studieprotocol van TELSTAR, een multicentrische gerandomiseerde klinische trial naar de behandeling van elektrografische status epilepticus na reanimatie. In dit lopende onderzoek beogen we 172 patiënten te includeren, die worden gerandomiseerd voor ofwel agressieve behandeling ofwel geen medicamenteuze behandeling van status epilepticus. ledere uitkomst van dit onderzoek zal leiden tot aanpassing van internationale richtlijnen, omdat beide behandelstrategieën momenteel worden beschouwd als standaardbehandeling. TELSTAR is gestart in 2014, en de inclusie zal naar verwachting in de tweede helft van 2019 worden afgerond. 


\section{Dankwoord (acknowledgements)}

Het is zover. Na vier jaar toewijding, een zinderende zomer en enkele werkdagen van 9:00 tot 5:00 is mijn proefschrift af. Ik wil graag iedereen bedanken die mij een zetje gaf richting de eindstreep van dit project.

Allereerst gaat mijn dank uit naar alle patiënten en hun naasten die aan het onderzoek hebben deelgenomen. Zonder $\mathrm{u}$ was dit proefschrift er niet geweest. $U$ besloot, veelal in een moeilijke situatie, uw toestemming te verlenen of informatie te verstrekken en daar ben ik ontzettend dankbaar voor.

Ik wil mijn promotor en co-promotor bedanken voor vier jaar intensieve begeleiding. Michel, het was bijzonder fijn om een promotor te hebben met een vergelijkbare achtergrond als ikzelf. Beiden hebben we geneeskunde en natuurkunde gestudeerd. Je natuurlijke enthousiasme voor wetenschap weet je uitstekend over te brengen op anderen. Jeannette, jij was altijd in staat om mijn werk binnen no-time te voorzien van rake feedback. Ik waardeer je no-nonsense aanpak, en bewonder je vermogen om feilloos tot de essentie van zaken door te dringen en deze glashelder te formuleren. Verder waardeer ik de vrijheid die jullie mij hebben geboden om zelf richting te geven aan mijn promotieonderzoek.

Ik dank de leden van mijn promotiecommissie voor het zorgvuldig lezen van mijn proefschrift en hun bereidheid om in de oppositie plaats te nemen. Ik kijk er naar uit om met u van gedachten te wisselen over mijn werk. Dr. Westover, many thanks for the careful review of my dissertation and your willingness to travel to the Netherlands for attending my defense.

Mijn proefschrift leunt voor een groot deel op de inzet van neurologen en intensivisten die zich naast de zorg voor patiënten inzetten voor wetenschappelijk onderzoek. Ik dank Walter van den Berg, Norbert Foudraine, Selma Tromp, Janneke Horn, Bert Kornips, Erik Scholten, Frank Bosch, Bert Beishuizen, Michiel Blans, Gea Drost en Fleur van Rootselaar voor het verzamelen van data, meedenken over het onderzoek en het kritisch lezen van mijn artikelen. Verder dank ik Alex Bos, Astrid Glimmerveen, Hanneke Keijzer en alle betrokken researchverpleegkundigen voor het verzamelen van klinische gegevens.

Ik wil iedereen bedanken die zich momenteel inzet voor de TELSTAR-studie, waaronder Jan Willem Elting, Monique Raaijmakers, Marjolein Admiraal, Antje Seeber, Elsbeth Thomeer, Annemieke Dijkstra, Vivianne van Kranen, Walther van Mook, Danny Hilkman, Astrid Hoedemaekers, Fabio Taccone, Nicolas Gaspard, Frouke Nijhuis, Hans Bernsen en Kuan Kho. Ik dank Bart van der Worp, Arjen Slooter en Maarten van Smeden voor het plaatsnemen in de DSMB. Hoewel het niet is gelukt de resultaten van TELSTAR in dit proefschrift op te nemen, ben ik er van overtuigd dat we met een laatste gezamenlijke inspanning tot een prachtig resultaat gaan komen. 
Naast de genoemde clinici ben ik veel dank verschuldigd aan Hil Meijer, die mij hielp om hoofdstuk 5 naar een hoger niveau te tillen. Hil, dank voor de fijne samenwerking. Ik voelde me altijd zeer welkom om samen met jou de details van de analyse uit te pluizen. Ik dank Joost le Feber en de vaste bezoekers van de tweewekelijkse Neuroscience Meeting, waaronder Stephan van Gils en Christoph Brune, voor waardevolle discussies en zinvolle feedback.

In mijn onderzoek heb ik gebruik gemaakt van bijna zes jaar EEG-registratie, gemeten met 18040 elektroden bij 902 patiënten. Hierachter gaat een enorme inspanning van KNF-laboranten schuil, in vijf verschillende ziekenhuizen. In het bijzonder dank ik Carin Eertman, die waarschijnlijk de meeste elektroden plakte van iedereen, ook bij nacht en ontij. Carin, ik bewonder jouw gedrevenheid en immer zonnige humeur. Je bent een geweldige ambassadeur van ons onderzoek in het Medisch Spectrum Twente.

Tijdens vier jaar op de Universiteit Twente heb ik vele collega's en studenten zien passeren. Annika, mijn collega-promovendus en paranimf, jij bent in al die jaren de stabiele factor geweest. Van de eerste rondleiding over de campus tot de dag van mijn promotie. Het was ontzettend fijn om te kunnen sparren met iemand in dezelfde fase van het promotieonderzoek. Ik kijk met plezier terug op onze gezamenlijke congresbezoeken, het organiseren van onderwijs en de vele koppen thee in de coffee-corner.

Goed onderzoek is alleen mogelijk als je kunt voortbouwen op het werk van anderen. Marleen, mijn voorganger in het onderzoek naar postanoxisch coma, dank voor al je hulp en tips in de beginfase van mijn promotietraject en daarna. Ik heb dankbaar gebruik gemaakt van het fundament dat jij hebt gelegd. Als co-auteur heb je aan twee hoofdstukken van dit proefschrift bijgedragen en ook voor de TELSTAR-studie heb je inmiddels veel werk verzet. Ik vind het leuk dat je als paranimf naast mij wilt staan.

Ik dank alle collega's van de vakgroep Clinical Neurophysiology voor vier gezellige jaren: Joost, Tanja, Gerco, Esther, Vesna, Anneloes, Bas-Jan, Irina, Marloes en Monica. Ik heb genoten van de congresbezoeken, de woensdagen met taart, de gezamenlijke kookworkshop en survival in de stromende regen. Alle collega's van ECTM en MDI, dank voor de dagelijkse gezelligheid tijdens de lunch en koffiepauzes. Alle master- en bachelorstudenten die in de afgelopen jaren passeerden, dank voor de frisse energie die jullie brachten binnen de vakgroep.

Sommige mensen verrichten op de achtergrond zoveel werk dat je het bijna niet opmerkt. Daarom noem ik ze graag. Tanja Gerrits en Jolanda Kuipers, dank voor alle secretariële ondersteuning en persoonlijke interesse en betrokkenheid, Henk van de Zandschulp voor het oplossen van ICT-problemen, Cindy Lammerlink voor het faciliteren van medisch-wetenschappelijk onderzoek op de UT en Wim Leppink voor de hulp bij alle praktische zaken waar niemand anders een oplossing voor heeft.

Ik wil alle vrienden bedanken die de aflopen jaren zorgden voor de nodige afleiding en ontspanning naast het werk. Adrien, Arend, Bart, Charlie, David, Duco, Hayko, Jesse, Jord, Maik, Mike, Mike, Pieter, Pim, Rei, Tibor, Vincent: in de afgelopen drukke maanden moest ik mijn sociale leven op een lager pitje zetten, maar dat ga ik zeker goedmaken. Jan, Vincent en Julia, het contact dat we hebben gehouden na onze natuurkundestudie vind ik erg waardevol. 
Papa en mama, dank voor jullie steun in de afgelopen jaren. Jullie hebben altijd onbevooroordeeld achter mijn keuzes gestaan, hoewel ze niet altijd logisch leken. Altijd kon ik bij jullie terecht voor een kop koffie, een borrel of een verse stapel Donald Ducks. Mijn lieve familie en schoonfamilie; Esther, Tom, Nynke, Jos, Gül, Anneke, Laura, Roy, Theo, Esther, Jasmijn, Rick, Sanne, Robbert: dank voor jullie interesse en betrokkenheid. Velen van jullie zijn in de afgelopen jaren betrokken geweest bij de zorg voor onze kinderen. In het bijzonder dank ik Anneke, Laura en mijn vader Joop, die hiervoor talloze treinreizen van Noord-Holland naar Enschede ondernamen.

De afgelopen vier jaar zijn voor mij intensief en bijzonder geweest. Niet alleen op wetenschappelijk, maar ook op persoonlijk vlak. Niels en Jasper, jullie werden tussen de hoofdstukken van dit proefschrift door geboren. Ik ben ontzettend blij met jullie komst. Ons samen verwonderen over de wereld om ons heen is het beste middel om al het andere te relativeren.

Lieve Corine, als ik een tweede naam aan het titelblad mocht toevoegen zou het de jouwe zijn. Je bent bij alle fases van mijn promotieonderzoek intensief betrokken geweest. Van chauffeur bij het sollicitatiegesprek tot adviseur bij het coverontwerp. Ik maakte het je niet gemakkelijk door je in mijn kielzog mee te slepen naar Enschede. Ik ben je enorm dankbaar dat je me desondanks altijd hebt gesteund. Ik kijk uit naar de toekomst met Niels, Jasper en jou.

Augustus 2018, Barry Ruijter 



\section{About the author}

Barry Johannes Ruijter was born on 19 September 1985 in Anna Paulowna. After graduating from high school in 2003, he studied medicine at the VU University in Amsterdam. In 2008, he also attended the University of Amsterdam to study physics and astronomy, and graduated with a bachelor's degree in 2010 (cum laude). Barry obtained his master's degree in medicine in 2012 (cum laude), after which he worked as a junior doctor in neurology and neurosurgery at the Medisch Centrum Alkmaar.

In 2014, Barry started his PhD project within the Clinical Neurophysiology group at the University of Twente, under supervision of prof. Michel van Putten and dr. Jeannette Hofmeijer. His project focused on validation and improvement of the value of continuous electroencephalography (EEG) for outcome prediction and treatment of comatose patients after cardiac arrest. His work comprised clinical studies, quantitative EEG analysis, and computational modeling. During his PhD project, Barry was involved in teaching bachelor's and master's students of technical medicine. 



\section{List of publications}

\section{Published}

Ruijter BJ, Hofmeijer J, Tjepkema-Cloostermans MC, Van Putten MJAM. The prognostic value of discontinuous EEG patterns in postanoxic coma. Clinical Neurophysiology 2018; 129: 1534-1543.

Hofmeijer J, Ruijter BJ, Tjepkema-Cloostermans MC, Van Putten MJAM. Early electroencephalography dynamics after cardiac arrest. Critical Care Medicine 2017; 45: e1093.

Ruijter BJ, Hofmeijer J, Meijer HGE, Van Putten MJAM, Synaptic damage underlies EEG abnormalities in postanoxic encephalopathy: a computational study. Clinical Neurophysiology 2017; 128: 1682-1695.

Van Putten MJAM, Hofmeijer J, Ruijter BJ, Tjepkema-Cloostermans MC. Deep Learning for outcome prediction of postanoxic coma. In: H. Eskola et al. (eds.), EMBEC \& NBC 2017, IFMBE Proceedings, vol 65. Springer, Singapore.

Sondag L, Ruijter BJ, Tjepkema-Cloostermans MC, Beishuizen A, Bosch FH, Van Til JA, Van Putten MJAM, Hofmeijer J. Early EEG for outcome prediction of postanoxic coma: prospective cohort study with cost-minimization analysis. Critical Care 2017; 21: 111.

Ruijter BJ, Van Putten MJAM, Hofmeijer J. Generalized epileptiform discharges in postanoxic encephalopathy: Quantitative characterization in relation to outcome. Epilepsia 2015; 56: 1845-1854.

Ruijter BJ, Van Putten MJAM, Horn J, Blans MJ, Beishuizen A, Van Rootselaar AF, Hofmeijer J. Treatment of electroencephalographic status epilepticus after cardiopulmonary resuscitation (TELSTAR): study protocol for a randomized controlled trial. Trials 2015; 15: 433.

\section{Submitted}

Tjepkema-Cloostermans MC, Hofmeijer J, Ruijter BJ, Beishuizen A, Bosch FH, Van Putten MJAM. Outcome prediction in postanoxic coma with deep learning (in revision).

Nagaraj SB, Tjepkema-Cloostermans MC, Ruijter BJ, Hofmeijer J, Van Putten MJAM. The revised Cerebral Recovery Index improves predictions of neurological outcome after cardiac arrest (in revision). 
Ruijter BJ, Tjepkema-Cloostermans MC, Tromp SC, Van den Bergh WM, Foudraine NA, Kornips FHM, Drost G, Scholten E, Bosch FH, Beishuizen A, Van Putten MJAM, Hofmeijer J. Early EEG for outcome prediction of postanoxic coma: a prospective multicenter cohort study (submitted).

Ruijter BJ, Van Putten MJAM, Van den Bergh WM, Tromp SC, Hofmeijer J. Propofol does not affect the reliability of early EEG for outcome prediction of comatose patients after cardiac arrest (submitted).

Glimmerveen A, Ruijter BJ, Keijzer HM, Tjepkema-Cloostermans MC, Van Putten MJAM, Hofmeijer J. Association between SSEP and EEG results in comatose patients after cardiac arrest (submitted). 

Each year, around 8000 people in the Netherlands are successfully resuscitated from cardiac arrest. Most of them remain comatose as a result of postanoxic brain injury, and are subsequently admitted to the intensive care unit. About half of these patients never regain consciousness, and treatment options to improve their outcome are limited. An important challenge is to identify those with and without chance of recovery. Recent work indicates that electroencephalography (EEG) within the first 24 hours after cardiac arrest allows for reliable predictions of outcome, even when patients are treated with hypothermia and sedative medication. The research described in this dissertation aims to validate the use of early continuous EEG for the prediction of outcome, using visual and quantitative methods. A computational model is presented that relates characteristic EEG abnormalities to mechanisms of postanoxic brain injury at the microscopic level. Finally, the dissertation includes the study protocol of the ongoing randomized TELSTAR trial, aimed at improving the outcome of patients in postanoxic coma with seizure-like EEG activity.

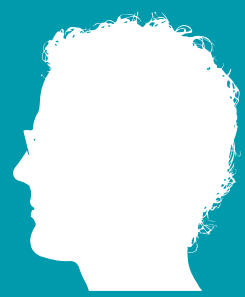

Barry Ruijter (1985) studied medicine at the VU University (MSc 2012) and physics at the University of Amsterdam (BSC 2010). Before starting his PhD project at the University of Twente in 2014, he worked as a junior doctor in neurology. 